\title{
Fundamentals of Phase Separation IN \\ Polymer Blend Thin Films
}

\section{Sam Coveney}

University of Sheffield

Department of Physics and Astronomy

Thesis prepared for the degree of Doctor of PhILOSOPhy

September 2014 



\section{Declaration of Authorship}

I, SAm Coveney, declare that the work presented in this thesis, except where otherwise stated, is based on my own research, guided by my Ph.D. supervisor Nigel ClaRke between 1st October 2011 and 30th September 2014, and has not been submitted previously for a degree in any university or institution.

Signed:

Date:

\section{Publications}

The work in this thesis has been published/submitted as follows:

1. Sam Coveney and Nigel Clarke. "Surface roughening in polymer blend thin films by lateral phase separation: A thermodynamic mechanism." In: The Journal of Chemical Physics 137 174901 (2012)

2. Sam Coveney and Nigel Clarke. "Breakup of a Transient Wetting Layer in Polymer Blend Thin Films: Unification with 1D Phase Equilibria." In: Physical Review Letters 111125702 (2013)

3. Sam Coveney and Nigel Clarke. "Lateral phase separation in polymer-blend thin films: Surface Bifurcation." In: Physical Review E 89062603 (2014)

4. Sam Coveney and Nigel Clarke. "Pattern Formation in Polymer Blend Thin Films: Surface Roughening couples to Phase Separation." In: Physical Review Letters 113218301 (2014) 


\section{Acknowledgements}

To my supervisor Nigel Clarke who always made time to help me, to the guys from the office who always put up with me, to the guys from aikido who always encouraged me, to my mum who always said she was proud of me, to my friends who always made allowances for me, and to Emily who was always there for me in all of these ways. 


\begin{abstract}
In this Ph.D. thesis, I investigate fundamental aspects of phase separation in polymer-blend thin films by unifying 1D phase equilibria with film evolution phenomena. I begin by extending a Hamiltonian phase portrait method, useful for visualising and calculating phase equilibria of polymer-blend films, allowing the method to be applied to systems with no convenient symmetries. Consideration of equilibria suggests a thermodynamic mechanism of film roughening, whereby laterally coexisting phases could have different depths in order to minimise free energy. I then make use of the phase portraits to demonstrate that simulations of lateral phase separation via a transient wetting layer, which conform very well with experiments, can be satisfactorily explained by 1D phase equilibria and a surface bifurcation mechanism involving effective boundary conditions caused by the film surfaces. Lastly, to tie together the aforementioned work, I introduce a novel 3D model of coupled phase separation and dewetting, for which I solved the problem of including a general non-uniform composition profile in the depth direction between the film surfaces. Pattern formation, in which surface roughening shadows the phase separation, seems to be determined by an interplay between dewetting kinetics and underlying phase equilibria.
\end{abstract}




\section{Preface}

Chapters 1 and 2 provide a chronological overview of the development of the base theory for phase separation in polymer-blend films used in this thesis. Chapter 1 focusses on the theory of bulk polymer systems, explaining the origin of a suitable form of free energy, phase diagrams and spinodal decomposition. Chapter 2 follows the extension of bulk theory to include preferentially attracting surfaces, including the origin of a form of surface energy, wetting of surfaces, and coexisting phases in films. Together, Chapters 1 and 2 cover the primary literature required to theoretically study polymer-blend thin films from a thermodynamic perspective.

Chapter 3 introduces the problem of solving for equilibrium profiles in polymerblend thin films. A Hamiltonian phase portrait method, previously only suitable for systems with particular symmetries, is extended to the general case of asymmetric polymer films, and a qualitative demonstration of how phase portraits can be used to study how equilibria change with film depth and temperature is given. A thermodynamic mechanism of surface roughening, whereby the depth of coexisting profiles can be different to reduce the free energy, is introduced.

In Chapter 4, Hamiltonian phase portraits and simulations of polymer-blend thin films are used to explain the phenomenon of lateral phase separation via a transient wetting layer. It is shown that films evolve first towards a metastable state (the lowest energy independently-existing equilibria) and then evolve towards global equilibrium (laterally coexisting phases). A novel 'surface bifurcation' mechanism, in which surface boundary conditions determines the particular way in which the transient wetting layer breaks up, is introduced to explain the observations from the simulations and spin coating experiments.

In Chapter 5, a novel 3D model of a phase separating polymer film that can undergo surface roughening via a dewetting mechanism is formulated. This formulation is made possible by solving the problem of including a general vertical dependence of the film composition in a dewetting model. This model is used to investigate surface roughening for films with different surface-blend interaction regimes, suggesting that surface pattern formation in polymer-blend thin films is general because surface roughening shadows the underlying phase separating morphology. The kinetics of dewetting appear to be as important as the underlying phase equilibria. I conclude this thesis with a summary and outlook.

I hope that I have written this thesis to be useful to another Ph.D. student. I have tried to include only the most relevant and primary literature, since it is my sincere opinion that broad and non-specific referencing is unhelpful to anyone new in the field. I hope that my schematics and explanations will transfer some of the imagery by which I negotiated this field to someone else. I have also included appendices on some technical aspects, namely the calculation of functional derivatives from first principles and implementations of diffusion simulations on Graphical Processing Units. Perhaps these will save someone else the time and energy of reinventing the wheel when they could be doing new physics. 


\section{Contents}

Authorship

Acknowledgements

Abstract $\quad$ v

Preface vi

1 Development of Theory for Bulk Polymer Blend Systems 1

1.1 Introduction . . . . . . . . . . . . . . . . . 2

1.2 Entropy of Mixing . . . . . . . . . . . . . . . . . . . . . 2

1.3 Heat of Mixing . . . . . . . . . . . . . . . . . . 6

1.4 Flory-Huggins Free Energy of Mixing . . . . . . . . . . . . . . . . 11

1.5 Flory-Huggins-de Gennes Free Energy . . . . . . . . . . . . . . . . 13

1.6 Spinodal Decomposition . . . . . . . . . . . . . . . . 18

1.7 Summary . . . . . . . . . . . . . . . . . 26

2 Development of Theory for Polymer-Blend Thin Films 27

2.1 Introduction . . . . . . . . . . . . . . . . 28

2.2 Non-uniform systems with a surface . . . . . . . . . . . . . 29

2.3 Form of the surface energy . . . . . . . . . . . . . . . . 30

2.4 Wetting in semi-infinite geometries . . . . . . . . . . . . 34

2.5 Phase Separation in finite geometries . . . . . . . . . . . . . . . . . . . . . . . . . . . . . . . . 47

2.6 Summary . . . . . . . . . . . . . . . . . . 44

3 Hamiltonian Phase Portraits for Polymer-Blend Thin Films $\quad 45$

3.1 Introduction . . . . . . . . . . . . . . . . . . 46

3.2 Phase Equilibria . . . . . . . . . . . . . . . . . . . . . . . . 49

3.3 Hamiltonian Phase Portraits . . . . . . . . . . . . . . . . 52

3.4 Phase Equilibria of Asymmetric Films . . . . . . . . . . . . . . 59

3.5 Surface Roughening . . . . . . . . . . . . . . . . . . . . 67

3.6 Summary . . . . . . . . . . . . . . . . . . 70

4 Lateral Phase Separation via Surface Bifurcation $\quad 71$

4.1 Introduction . . . . . . . . . . . . . . . . . . 72

4.2 Calculating phase equilibria in $1 \mathrm{D} \ldots \ldots . \ldots 75$

4.3 Modelling Phase Separation . . . . . . . . . . . . . . . . 77

4.4 1D equilibria in 2D films . . . . . . . . . . . . . . . 83

4.5 Breakup of a Transient Wetting Layer . . . . . . . . . . . . . 90

4.6 Discussion . . . . . . . . . . . . . . . . . . . . . . . . 102

4.7 Summary . . . . . . . . . . . . . . . . . . . 104 
5 Coupled Surface Roughening and Phase Separation 107

5.1 Introduction . . . . . . . . . . . . . . . . . . . . 108

5.2 A 3D Model . . . . . . . . . . . . . . . . . . . 111

5.3 Application to polymer-blend thin films . . . . . . . . . . . . 116

5.4 Results and Discussion . . . . . . . . . . . . . . . . . . 118

5.5 Summary . . . . . . . . . . . . . . . . . . . . . 125

$\begin{array}{ll}\text { Summary of Research and Outlook } & 127\end{array}$

$\begin{array}{ll}\text { Appendices } & 131\end{array}$

A Diffusion Simulations on GPUs with CUDA 133

A.1 Principles of Parallelised GPU code . . . . . . . . . . . . . 133

A.2 Steps of Simulation . . . . . . . . . . . . . . . . . . . . . 134

A.3 Improving Efficiency . . . . . . . . . . . . . . . . . . 137

B Functional Derivatives for dewetting model 141

B.1 'Continuous derivation' . . . . . . . . . . . . . . . . . . 141

B.2 'Discrete derivation' . . . . . . . . . . . . . . . . . . . . 145

C Numerical implementation for 3D dewetting model 149

C.1 Lateral Transport Step . . . . . . . . . . . . . . . . . . . . . 150

C.2 Diffusion Step . . . . . . . . . . . . . . . . . . 152

$\begin{array}{ll}\text { Terminology } & 153\end{array}$

$\begin{array}{lr}\text { Publications } & 156\end{array}$

$\begin{array}{ll}\text { Bibliography } & 157\end{array}$ 


\section{DEVELOPMENT OF THEORY FOR}

\section{Bulk Polymer Blend Systems}

I follow the development of theory for solutions and blends of polymers. I take a minimal historical approach by focussing on the primary literature in which the theory was developed, and show how the work culminated ultimately in the Flory-Huggins-de Gennes free energy of mixing, which is the base theory for the study of spinodal decomposition in polymer blends.

1.1 Introduction . . . . . . . . . . . . . . . . 2

1.2 Entropy of Mixing . . . . . . . . . . . . . . . . 2

1.3 Heat of Mixing . . . . . . . . . . . . . . . . . . . . 6

1.4 Flory-Huggins Free Energy of Mixing . . . . . . . . . . . . . . . . 11

1.5 Flory-Huggins-de Gennes Free Energy . . . . . . . . . . . . . . 13

1.6 Spinodal Decomposition . . . . . . . . . . . . . . . . . 18

1.7 Summary . . . . . . . . . . . . . . . . . . . 26 


\section{$1.1 \quad$ Introduction}

The aim of this chapter is to provide an overview of the development of theory for bulk polymer ${ }^{1}$ systems, which came from a drive to understand the behaviour of solutions ${ }^{2}$ and blends $s^{3}$ of polymers, which differed significantly from the behaviour of non-polymer systems. I take a minimal historical approach to this, using what I regard to be the most important literature in which the theory was developed, to give a narrative to the development of the theory. This chapter can be summarised in the following. The behaviour of polymers in solution prompted the development of an entropy of mixing valid for long chain molecules. To fit the theory to data required an empirical term to account for the heat of mixing, the form of which was quickly grounded theoretically. The entropy of mixing and heat of mixing can be combined, along with a term accounting for energy contributions from compositional gradients, to give the Flory-Huggins-de Gennes free energy of mixing, which can be used to understand and study spinodal decomposition of polymer blends.

It is useful at this point to introduce the Gibbs free energy, which is appropriate when considering incompressible systems (although the assumption of constant volume is of course not general). Since the subject matter of this chapter is mainly changes upon mixing, we can consider the Gibbs free energy change, given by

$$
\Delta G=\Delta H-T \Delta S,
$$

where $\Delta H$ is the Heat (Enthalpy) of Mixing, $\Delta S$ is the Entropy of Mixing, and $T$ is the Temperature. I will refrain from elaboration of standard thermodynamics terminology throughout.

\section{Terminology}

I will briefly introduce terms as they appear, but more detailed definitions of Terminology are given on page 153. There are several terms that are used in passing while discussing literature in this section, and those that are not specifically important to this thesis will not be explicitly defined; definitions can be found elsewhere and in the corresponding citations.

\section{$1.2 \quad$ Entropy of Mixing}

By 1940 there was a substantial body of evidence showing that polymer solutions deviated significantly from Raoult's law (Eq. (1.8)), which describes how the vapour pressure of an ideal solution (zero heat of mixing $\Delta H=0$ ) depends on the vapour pressure of the pure components of the solution and the molar fraction of those components in the solution. These deviations were initially, and

\footnotetext{
${ }^{1}$ Polymer: a molecule consisting of repeated units, like a string of beads or a chain. These repeat units are called monomers. A chain segment usually refers to a single monomer.

${ }^{2}$ Solution: a liquid mixture of solvent (e.g. water, toluene) and solute (e.g. sugar, polymer), in which the solute is dispersed in the solvent.

${ }^{3}$ Blend: a liquid mixture of two components (e.g. a blend of two polymers).
} 

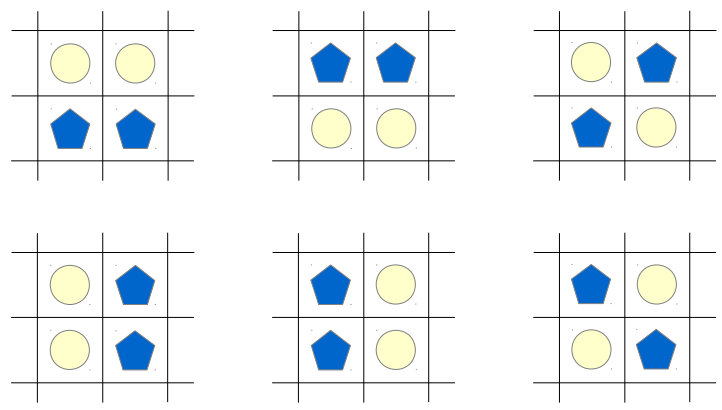

Figure 1.1: A blend $A B$ on a quasi-solid lattice. There are $n=4$ simple molecules, $n_{A}=2$ and $n_{B}=2$, hence the number of distinguishable configurations is $\Omega=$ $4 ! / 2 ! 2 !=n ! / n_{A} ! n_{B} !=6$. All six distinguishable configurations are shown.

almost exclusively, put down to enthalpic effects: it was assumed that a non-zero heat of mixing was causing the deviations from Raoult's Law. However, careful experiments showed that deviations from Raoult's Law were significant even when the heat of mixing really was zero. The first successful efforts to explain these deviations were undertaken by Huggins $[1,2]$ and Flory [3], who derived a form for the entropy of mixing suitable for polymers.

\subsubsection{Entropy of Ideal Solutions}

Consider a mixture $A B$ of fluids $A$ and $B$, consisting of equal sized simple molecules ${ }^{4}$. An ideal solution has zero heat of mixing, which means that there is no difference in the enthalpic interactions $U$ between molecules of the pure components ( $A-A$ and $B-B$ interactions) and between molecules of different components ( $A$ - $B$ interactions) i.e. $2 U_{A B}=U_{A A}+U_{B B}$. This means that the molecules will randomly mix to maximise entropy, since there are no particularly favourable or unfavourable interactions that would prevent an entirely random mixing.

The entropy of the mixture is given by the Boltzmann equation

$$
S=k_{B} \ln \Omega,
$$

where $\Omega$ is the number of distinguishable configurations of the mixture. To calculate $\Omega$, we can place each molecule on a quasi-solid lattice. If the molecules of fluids $A$ and $B$ are the same size, then the number of configurations available to $n=n_{A}+n_{B}$ molecules is $n$ !, but the number of distinguishable configurations is

$$
\Omega=\left(n_{A}+n_{B}\right) ! / n_{A} ! n_{B} ! .
$$

A schematic of a set of available configurations is shown in figure 1.1.

Using Eq. (1.2), we can find the change of entropy upon mixing as the difference in entropy between the mixture and the pure components, $\Delta S_{m i x}=$ $S_{A B}-S_{A}-S_{B}$, giving the entropy of mixing per molecule as

$$
\Delta S_{m i x}=-k_{B}\left[x_{A} \ln x_{A}+x_{B} \ln x_{B}\right],
$$

\footnotetext{
${ }^{4}$ Simple Molecules: molecules that can be treated as spheres, because they consist of a few atoms at most and their internal structure need not be explicitly considered.
} 
where $x_{A}=n_{A} / n$ and $x_{B}=n_{B} / n$ are molar fractions of $A$ and $B$ respectively. The entropy change $\Delta S_{\text {mix }}$ is a configurational entropy, because it only accounts for entropy changes due to the change of available configurations upon mixing. Strictly speaking this expression only applies to mixtures in which the molecules of both species are interchangeable, i.e., equal sizes and interaction energies; this means a molecule of $A$ can be swapped with a molecule of $B$ with no penalty.

A regular solution is one in which the entropy of mixing is given by equation (1.4), as for an ideal solution, but with $\Delta H \neq 0$. That polymer solutions do not obey Raoult's Law even when there was zero heat of mixing meant that polymer solutions are non-regular solutions.

\subsubsection{Entropy of Polymer Solutions}

The derivation of an entropy of mixing appropriate for polymer solutions was undertaken separately by Huggins [2] and Flory [3], and although both derivations were published in 1942, it was Huggins who published a brief letter of his results the previous year [1], in which it was stated that "in solutions of long, flexible chain molecules, deviation in the entropy of mixing from that given by [equation (1.4)] may be even more important (than the enthalpy of mixing effects)". Meyer is credited by Flory with the suggestion that the entropy of mixing for polymer containing systems must be responsible for these discrepancies, due to the intrinsic connectivity of polymer chains [3].

Flory explicitly laid down the assumptions required for the derivation [3]:

(i) assume a quasi-solid lattice in the liquid and interchangeability of polymer segments with solvent molecules (same assumptions used to derive equation (1.4)). A segment is defined as being equal in volume and shape to a solvent molecule;

(ii) all polymer molecules are the same size (although in 1944 Flory showed that "heterogeneity can be disregarded", since using a number average of chain lengths in a distribution will include the effects of heterogeneity [4]);

(iii) "the average concentration of polymer segments in cells adjacent to cells unoccupied by the polymeric solute is taken to be equal to the over-all average concentration", which is a mean-field assumption (this can let the theory down severely under certain conditions e.g. in very dilute solutions in which solute can clump together);

(iv) we don't consider that the chain might curve around and cross itself once again, which Flory noted would "(obviously) lead to computation of too many configurations".

Here I will give a simplified explanation in the spirit of the aforementioned references. Figure 1.2 is a schematic to assist in following the explanation. We assume a polymer chain to consist of $x$ segments $(x=5)$. Given $n_{s}$ solvent molecules $\left(n_{s}=27\right)$ and $n_{p}$ polymer molecules $\left(n_{p}=3\right)$, we require $n_{s}+x n_{p}$ lattice cells $\left(n_{s}+x n_{p}=27+(3 \times 5)=45\right)$. We then place, at random, the 
end segment of a single polymer chain on a lattice cell, hence there are $n_{s}+x n_{p}$ possible configurations for this move. The next segment from the same chain has much less freedom, of course, because it is connected to the first segment. Given this restriction, this segment has $z$ sites to choose from, where $z$ is the coordination number this gives the second segment $z$ sites to choose from (in this case, perhaps $z=5$, since there are five neighbouring sites to choose from; this drops out of the resulting expression). However, this second segment doesn't really have this much choice, since if the polymer chain were part of a filled lattice, there might already be segments from another chain next to the first segment of the chain we are considering. Using assumptions (iii) and (iv), we assume that we may put the number of configurations for the second segment to be $z\left(1-f_{p}\right)$ where $f_{p}$ is the probability that a cell is already occupied ( $f_{p}$ also drops out of the final expression). Once all polymer chains have been placed on the lattice, the remaining sites are filled with solvent molecules. Counting up all the configurations available, and subtracting the entropy of the pure states of both polymer and solvent, we arrive at

$$
\begin{aligned}
\Delta S_{\text {mix }} & =-k_{B}\left[n_{s} \ln \frac{n_{s}}{n_{s}+x n_{p}}+n_{p} \ln \frac{x n_{p}}{n_{s}+x n_{p}}\right] \\
& =-k_{B}\left[n_{s} \ln (\phi)+n_{p} \ln (1-\phi)\right],
\end{aligned}
$$

where $\phi$ is the volume fraction of solvent, therefore $1-\phi$ is the volume fraction of polymer.

Although in (i), we defined a segment as being equal in size to a solvent molecule, it may be necessary that a segment in the polymer chain is necessarily the size of several solvent molecules, since a segment must be at least so big as to allow the chain complete flexibility around these segments. In this case, we should define the lattice cell to be the size of the segment, and have several solvent molecules to one cell. Flory addressed this [3], arguing that this can be accounted for by the rescaling $n_{s} \rightarrow n_{s} / \beta, x \rightarrow x / \beta$ where $\beta$ is the number of solvent molecules that will fill a cell the volume of a single polymer segment.

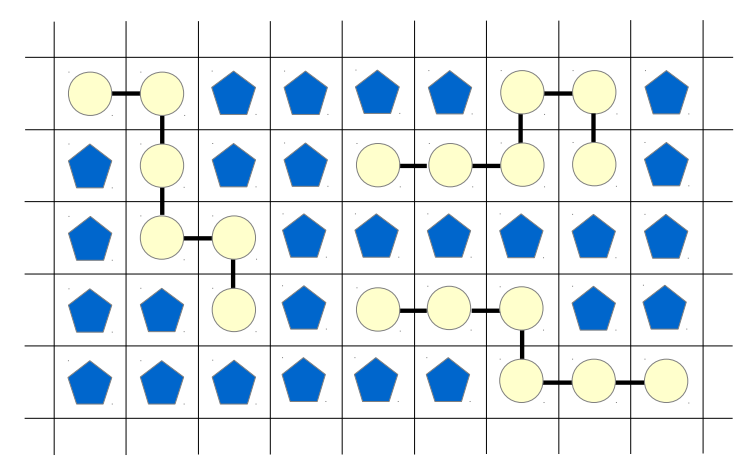

Figure 1.2: A schematic of a quasi-solid lattice, on which 3 polymer chains (6 segments longs) have been placed, and the remaining lattice cells filled with solvent molecules. The polymer chains require connectivity. 
This simply re-enforces the requirement to correctly measure the polymer chains in terms of segment lengths / lattice spacing (so a polymer chain may consist of 15 repeat units / monomers, but a segment may consist of 3 monomers, hence the chain is 5 segments long).

It is more natural to express this equation per 'molecule', where the number of molecules equals the number of lattice cells $n_{s}+x n_{p}$. We arrive at

$$
\Delta S_{m i x}=-k_{B}\left[\phi \ln (\phi)+\frac{(1-\phi)}{x} \ln (1-\phi)\right]
$$

where $\Delta S_{m i x}$ has been redefined as the entropy of mixing per molecule. This equation can be generalised to polymer-polymer mixtures. If the solvent is replaced by polymer species $A$ with $y$ number of segments, then the factor of $\phi$ can be replaced by $\phi / y$ in the first term. It is more natural to replace $y$ with $N_{A}$ and $x$ with $N_{B}$, where $N_{i}$ represents the number of segments in species $i$ (the segment size of both species being chosen to be equal in the definitions of $N_{i}$ ). This gives

$$
\Delta S_{m i x}=-k_{B}\left[\frac{\phi}{N_{A}} \ln (\phi)+\frac{(1-\phi)}{N_{B}} \ln (1-\phi)\right] .
$$

Equation (1.7) is known as the Flory-Huggins Entropy of Mixing. Notice that unlike equation (1.4), the logarithm terms contain volume fractions. If $N_{A}=$ $N_{B}=1$ then equation (1.7) reduces to equation (1.4) for ideal solutions.

Although any lattice parameters do not strictly appear in (1.7), it is worth noting again that the 'length' of a polymer species should be counted in units of lattice size. So if species $A$ and $B$ have the same number of monomer units and are both flexible around these units, then if the size of $A$-monomers are twice the size of $B$-monomers, we have $N_{A}=2 N_{B}$ (assuming the lattice cells are the size of the A-monomers, which is required to allow the $A$-chains to be flexible). Working in volume fractions $\phi$ accounts for the other mathematical difference due to B-chains having half the volume of A-chains.

\subsection{Heat of Mixing}

Although deviations from Raoult's law could be shown to derive from the entropy of mixing given by equation (1.5), fits to the activities data still require a term that took the heat of mixing into account [5]. Of course, generally a heat of mixing term for polymers will be required, because the heat of mixing is rarely zero.

\subsubsection{Activities Data}

Raoult's law relates the vapour pressure of an ideal solution to the vapour pressure of each solution-component and the mole fraction of that component. Huggins used an expression essentially equivalent to Raoult's Law, writing the chemical potential $\mu_{i}$ of species $i$ in a solution as [5]

$$
\mu_{i}=\mu_{i}^{o}+R T \ln a_{i}
$$


where the reference state with chemical potential $\mu_{i}^{o}$ may refer to the pure component, for simplicity. The 'activity' is defined as $a_{i}=p_{i} / p_{o}$, where $p_{i}$ and $p_{o}$ are the vapour pressures of component $i$ in the solution and as pure component, respectively. An expression for the difference in chemical potential can be found from the entropy of mixing:

$$
\Delta \mu_{p}=-\frac{\partial(T \Delta S)}{\partial n_{p}^{*}},
$$

where $n_{p}^{*}$ is now the number of moles of polymer, and $\Delta \mu_{p}=\mu_{p}-\mu_{p}^{o}$. The entropy of mixing (1.5) in terms of the number of moles of solvent and polymer is then

$$
\Delta S_{m i x}=-R\left[n_{s}^{*} \ln \frac{n_{s}^{*}}{n_{s}^{*}+x n_{p}^{*}}+n_{p}^{*} \ln \frac{x n_{p}^{*}}{n_{s}^{*}+x n_{p}^{*}}\right] .
$$

Using equation (1.9) and converting back into volume fractions, we arrive at

$$
\frac{\Delta \mu_{p}}{R T}=\ln a_{p}=\ln \phi_{p}+(1-x) \phi_{s} .
$$

From the way the number of segments $x$ in the polymer molecules is defined, $x$ can be written in terms of a ratio of volumes of the polymer and solvent molecules $x=\bar{V}_{p} / \bar{V}_{s}$. Generalising to polymer-polymer systems (since we can always choose $N=1$ for either polymer for it to be a simple solvent), there are two expressions for a binary mixture

$$
\begin{aligned}
& \ln a_{A}=\ln \phi_{A}+\left(1-\frac{\bar{V}_{A}}{\bar{V}_{B}}\right) \phi_{B}, \\
& \ln a_{B}=\ln \phi_{B}+\left(1-\frac{\bar{V}_{B}}{\bar{V}_{A}}\right) \phi_{A},
\end{aligned}
$$

where either $A$ or $B$ could be a polymeric solute or a solvent.

The osmotic pressure of the solvent can be related to the activity by

$$
\frac{\Pi}{c_{s}}=-\frac{R T}{c_{s}^{2}} \ln a_{s},
$$

where $c_{s}$ is the concentration of polymer solute or equivalently (given different units) the partial molar volume. In order to account for how, in polymer solutions, $\Pi / c_{s}$ increases with $c_{s}$ Huggins needed to include an empirical term in equations (1.12) which "takes care of the heat of mixing, deviations from complete randomness of mixing, and other factors" [5]:

$$
\begin{aligned}
& \ln a_{A}=\ln \phi_{A}+\left(1-\frac{\bar{V}_{A}}{\bar{V}_{B}}\right) \phi_{B}+\mu_{A} \phi_{B}^{2}, \\
& \ln a_{B}=\ln \phi_{B}+\left(1-\frac{\bar{V}_{B}}{\bar{V}_{A}}\right) \phi_{A}+\mu_{B} \phi_{A}^{2} .
\end{aligned}
$$

Using equations (1.13) and (1.14) Huggins showed that the expression for the entropy, equation (1.7), fit data on polymer solutions, providing the empirical 
constants $\mu_{A}$ and $\mu_{B}$ are chosen suitably for a particular solution (with the condition that $\mu_{A} \bar{V}_{A}=\mu_{B} \bar{V}_{B}$, which is natural since the heat of mixing is a mutual interaction between opposing species and must be balanced). In hindsight, the need for the empirical constants to be included in equation (1.14) can be seen to arise from the definition of the chemical potential (1.9), since the full expression should be $\Delta \mu_{p}=\partial(\Delta G) / \partial n_{p}^{*}$. However, the form of $\Delta H$ was not yet known.

\subsubsection{A van Laar form for the heat of mixing}

Flory provided a simple derivation for an appropriate form for the heat of mixing [4]. The result is the van Laar expression for the heat of mixing of simple molecules, which has a simple lattice-based explanation [6], which follows. If a fluid $A$ and fluid $B$, both consisting of simple molecules, occupy molar volumes $v$ and $V$ respectively, then for a solution of $n$ moles of $A$ and $N$ moles of $B$, the internal energy per mole of solution can be written as

$$
U_{A B}=\frac{\epsilon_{A A}(v n)^{2}+2 \epsilon_{A B}(v n V N)+\epsilon_{B B}(V N)^{2}}{v n+V N} .
$$

Subtracting the energy of (the same quantity of) the pure fluids $U_{A}=\epsilon_{A A} v n$, $U_{B}=\epsilon_{B B} V N$, and gathering terms, gives

$$
\begin{aligned}
& \Delta U=\Delta \epsilon \frac{v V n N}{n v+N V}, \\
& \Delta \epsilon=2 \epsilon_{A B}-\epsilon_{A A}-\epsilon_{B B} .
\end{aligned}
$$

For polymer systems, the argument can be made that the form of interactions between polymer segments and solvent molecules should be the same as those between simple molecules. Assuming no volume change upon mixing, $\Delta H=\Delta U$, so the partial molal heat of $A$, given by $\Delta \bar{H}_{A}=\partial \Delta H / \partial n$, is then

$$
\Delta \bar{H}_{A}=\Delta \epsilon \phi_{B}^{2}
$$

which is exactly the same form as the heat of mixing term in equation (1.14). However, Flory was quick to point out that the use of this term provides satisfactory agreement with experiment, but that it clearly must contain "contributions from other factors the origins of which are not yet clear" [4]. This could include, of course, entropy effects due to the heat of mixing and configurational entropy modifications to equation (1.7) from the fact that, given a finite heat of mixing, systems of polymers and solvents will not be entirely uniform.

\subsubsection{The Flory-Huggins Interaction Parameter}

A heat of mixing consistent with equation (1.16) can be derived from a general lattice model with coordination number $z$, as in Flory's textbook [7]. However, I found the latter derivation slightly difficult to follow, so I have opted to derive the heat of mixing in line with a more modern approach [8]. 
A mean-field ${ }^{5}$ assumption can be applied to a binary polymer mixture $A B$ on a quasi solid lattice, and assume that the probability that a lattice cell picked at random will contain a segment of $A$ or $B$ is given by the volume fraction of $A$ or $B$, denoted by $\phi_{A}$ or $\phi_{B}$ respectively. Also, given this chosen site, the probability that any neighbouring site contains a segment of $A$ or $B$ is also given by $\phi_{A}$ or $\phi_{B}$ respectively. If the interaction energy between two $A$ segments is $\epsilon_{A A}$, then given the probability of choosing an $A$-segment when choosing the first site is $\phi_{A}$, and given that the probability of a neighbouring site containing an A-segment is $\phi_{A}$, then the contribution to the average site energy from $A$ - $A$ interactions will be $\epsilon_{A A} \phi_{A}^{2}$. The average energy of a site can then be given by the general formula

$$
U_{\text {site }}=z \sum_{i=A, B} \sum_{j=A, B} \epsilon_{i j} \phi_{i} \phi_{j}
$$

whereas the total energy of the pure states of $A$ and $B$ is given by

$$
U_{\text {pure }}=z \sum_{i=A, B} \epsilon_{i i} \phi_{i}
$$

Performing $U_{\text {site }}-U_{\text {pure }}$ gives the change in internal energy upon mixing per site. Assuming no volume change, this is the same as the enthalpy of mixing.

$$
\begin{aligned}
\Delta H_{m i x} & =k_{B} T \chi \phi_{A} \phi_{B}, \\
\chi & =z \Delta \epsilon / k_{B} T \\
\Delta \epsilon & =2 \epsilon_{A B}-\epsilon_{A A}-\epsilon_{B B} .
\end{aligned}
$$

Equation (1.21) is almost exclusively used to represent the heat of mixing. The dimensionless parameter $\chi$ is called the Flory-Huggins interaction parameter. It can be measured in experiments, and is usually considered to be an experimental parameter to describe the heat of mixing without reference to any microscopic effects or lattice theory model. However, in this particular lattice theory model from which $\chi$ has been explained, $\chi$ is purely enthalpic in origin. An entropic contribution is generally necessary.

\section{Non-combinatorial entropy}

The entropy of mixing (1.7) represents the combinatorial entropy of mixing, resulting only from the change in available configurations for non-interacting chains (in other words, it arises from the increased volume in which the polymer molecules can distribute themselves, which allows them access to more configurations [9]). In general, we should expect $\chi$ to have an entropic part too, usually referred to as a non-combinatorial entropy, and may arise from the non-uniformity of a solution caused by preferential attraction between like components, or from a change in the accessibility of energy levels or restriction of certain rotational configurations due to interactions.

\footnotetext{
${ }^{5}$ Mean-field: average interactions are used in place of counting up individual interactions, such that the local behaviour can be written in terms of macroscopic average properties.
} 
The entropy of mixing can be obtained from equation (1.1) as

$$
\Delta S=-\frac{\partial \Delta G}{\partial T}
$$

and the enthalpy/heat of mixing as

$$
\Delta H=\Delta G+T \Delta S
$$

Substituting in the entropy of mixing (1.7) and the heat of mixing (1.21), and assuming that it is possible that $\chi$ depends on temperature, gives

$$
\Delta S=-k_{B}\left[\frac{\phi}{N_{A}} \ln (\phi)+\frac{(1-\phi)}{N_{B}} \ln (1-\phi)+\phi(1-\phi) \frac{\partial(\chi T)}{\partial T}\right] .
$$

From this follows that

$$
\Delta H=\Delta G+T \Delta S=k_{B} T \phi_{A} \phi_{B}\left(\chi-\frac{\partial(\chi T)}{\partial T}\right)
$$

Comparing this with the heat of mixing (1.21) we see that, in general, the FloryHuggins interaction parameter $\chi$ has both an enthalpic and entropic part $[7,10]$, such that $\chi=\chi_{H}+\chi_{S}$, where

$$
\begin{aligned}
\chi_{H} & =\chi-\frac{\partial(\chi T)}{\partial T}=-T \frac{\partial \chi}{\partial T}, \\
\chi_{S} & =\frac{\partial(\chi T)}{\partial T} .
\end{aligned}
$$

Thus in order for the interaction parameter to be purely enthalpic, it must have temperature dependence $\chi \propto 1 / T$.

Anomalous contributions to the entropy of mixing were often put down to changes in volume which the lattice model used to derive equation (1.7) cannot include. Whilst changes in volume will of course alter the entropy, numerous experiments under fixed volume still show that there is a contribution to the entropy upon mixing that cannot be accounted for by equation (1.7) and thus a non-combinatorial entropy contribution must exist [9]. This idea is now a standard part of the literature [11].

\section{Dependence of heat of mixing on volume fraction}

In Flory's first paper on the subject [3] it was suggested that the agreement between theory and experiment would be better if the enthalpy term equivalent to $\Delta \epsilon$ in equation (1.18), which acts as an analogue of $\chi$, was given an appropriate dependence on concentration. For the rubber-toluene solution measurements in question, the theory was rather accurate for high concentrations of rubber solute, but matched the data at low rubber concentrations only with an empirical fit for the heat of mixing. In [4], Flory returned to this matter, mentioning that the fit that Huggins had made to a benzene-rubber solution (which required no concentration dependence for the empirical terms containing $\mu_{i}$ ) was correct, 
but that the matter was actually more complicated. Other measurements that separately measured the heat of mixing and entropy of mixing in this system confirmed that both departed significantly from the theory, but "when these two somewhat erroneous equations are combined, however, a satisfactory free energy function is obtained, as Huggins has shown". Flory suggested that a finite heat of mixing might be responsible, since this would necessarily lead to non-uniform mixing (clusters of solute in pure solvent).

This idea was explicitly addressed by Flory in a paper soon after [12], in which Flory investigated the case of highly diluted polymer solutions. Experiments showed that the heat of dilution was dependent on the concentration of polymer solute, and there was a marked difference between dilute and concentrated solutions. The heat of mixing as given by the van Laar form in equation (1.14) could be reconciled with the data provided that $\mu$ is reformulated as

$$
\mu=\beta+\alpha / R T
$$

in which both $\alpha$ and $\beta$ depend on the concentration. Flory states that the benzene-rubber system analysed by Huggins is essentially a special case in which the free energy function does not require $\mu$ to depend on concentration, even though the entropy and heat of dilution equations when considered separately do not match the data. Flory points out that the value of $\mu$ needed for the fit is actually much lower than theory would predict, which indicates that $\mu$ is really just an empirical constant, and that "in spite of the approximate constancy of $\mu$ for rubber in benzene at all concentrations, it is unlikely that this condition applies to high polymer solutions in general". Flory showed that a different $\mu$ was required for solutions of high concentration than low concentration, and the constant $\alpha$ must change and, generally, it is "likewise necessary to throw the burden of $\mu$ on $\beta$ in dilute solutions" [12]. Eq. (1.28) is essentially equivalent to the modern common expression for the Flory-Huggins parameter:

$$
\chi=A+\frac{B}{T} .
$$

\section{$1.4 \quad$ Flory-Huggins Free Energy of Mixing}

Substituting the Entropy of Mixing (1.7) and the Heat of Mixing (1.21) for polymer systems into the expression for the Gibbs free energy (1.1), we obtain the Flory-Huggins Free Energy of Mixing $f_{F H} \equiv \Delta F_{m i x}=\Delta H_{m i x}-T \Delta S_{m i x}$. In units of $k_{B} T$, we can write

$$
f_{F H}(\phi)=\frac{\phi}{N_{A}} \ln (\phi)+\frac{(1-\phi)}{N_{B}} \ln (1-\phi)+\chi \phi(1-\phi) .
$$

The expression $f_{F H}(\phi)$ is the 'bulk' free energy for a polymer blend, giving the free energy per lattice site in the Flory-Huggins lattice with spacing $a$. 


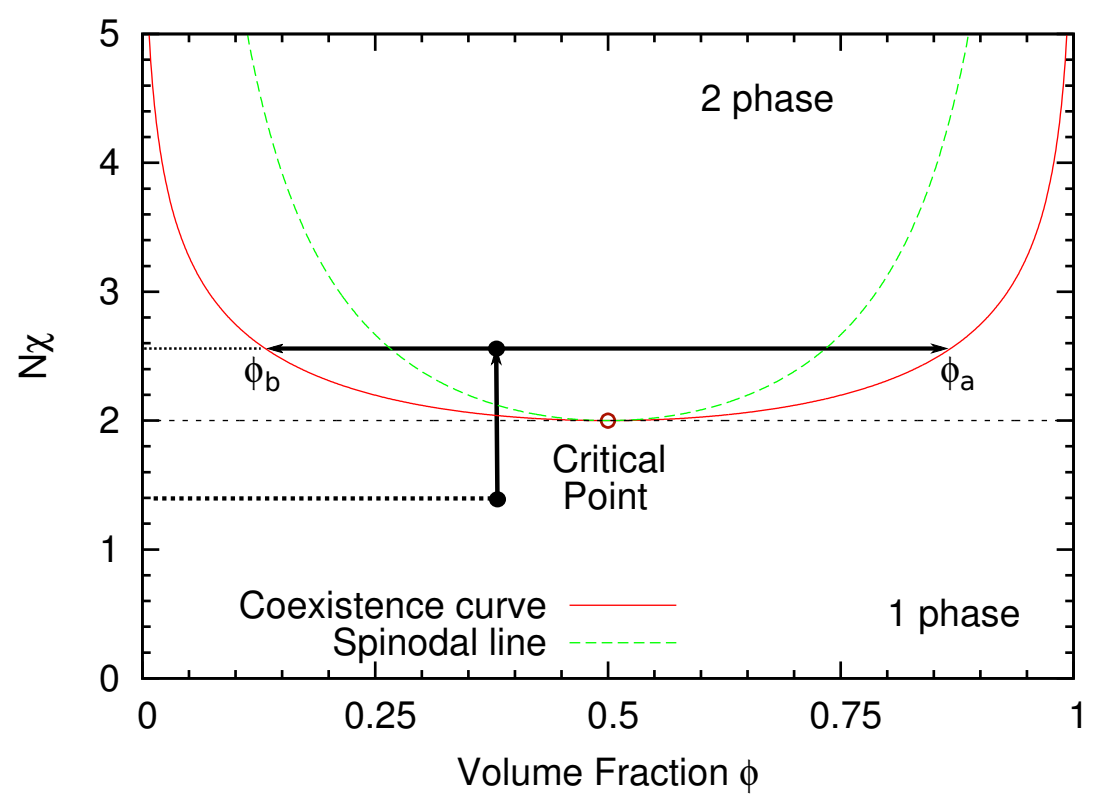

Figure 1.3: Phase diagram in the $\phi-\chi$ plane (essentially equivalent to compositiontemperature) for a polymer blend $N=N_{A}=N_{B}$. Below the coexistence curve, it is favourable for the polymer blend to remain mixed, hence 1-phase is stable. Above the coexistence curve, it is favourable for the polymer blend to de-mix, hence 2-phases are stable. Between the coexistence curve and the spinodal line, 1-phase has more energy than 2-phases, but 1-phase is metastable, and so 1phase may still exist in this region. So the spinodal line represents the limit of stability for the blend remaining in the 1-phase state i.e. above the spinodal, 1phase is unstable. The critical point $\left(\phi_{C}, \chi_{C}\right)$, located at critical volume fraction $\phi_{C}$ and critical temperature $\chi_{C}$, corresponds to where the coexistence curve and spinodal line coincide. It is the first point at which the blend becomes unstable upon increasing $\chi$ (assuming $\chi=A+B T^{-1}$, then the critical point marks the highest temperature for which a blend in the 1-phase region is unstable).

\section{Phase diagram from the Flory-Huggins free energy}

Equation (1.30) can be used to compute a phase diagram $^{6}$ for the blend which separates the one-phase region (the components of the polymer blend remains mixed together, entropy overcoming enthalpy) from the two-phase region (the polymer blend de-mixes into two phases, each rich in one component of the polymer blend) in the plane of composition and temperature. Figure 1.3 is a phase diagram for the polymer blend $N=N_{A}=N_{B}$, containing a coexistence curve and spinodal line, explained below.

The limits of stability of a polymer blend can be calculated by consideration of the first and second derivatives of the free energy (1.30) with respect to composition, $d F / d \phi$ and $d^{2} F / d \phi^{2}$ respectively. To demonstrate, I will consider a

\footnotetext{
${ }^{6}$ Phase Diagram: a diagram, drawn in a space of variables such as composition and temperature, that separates regions corresponding to different stable phases with lines, which correspond to the limits of stability of these phases. e.g. for water, a phase diagram in the temperaturepressure plane separates regions of vapour, liquid and solid.
} 
blend in which the two polymers $A$ and $B$ have the same chain lengths (degree of polymerisation) $N_{A}=N_{B}=N$, since this is the simplest example. The first derivative is

$$
\frac{\partial F}{\partial \phi}=\frac{1}{N} \ln \left(\frac{\phi}{1-\phi}\right)+\chi(1-2 \phi)
$$

$d F / d \phi=0$ corresponds to minima in the free energy, and we can rearrange the resulting expression so that we can plot a locus of points for which $d F / d \phi=0$, giving us the 'coexistence curve'

$$
\chi_{\text {coex }}=\frac{1}{N} \frac{1}{2 \phi-1} \ln \left(\frac{\phi}{1-\phi}\right) .
$$

(If the blend is not symmetric, then calculating the coexistence curve is more complicated, requiring equating the chemical potentials of both species). The second derivative is

$$
\frac{\partial^{2} F}{\partial \phi^{2}}=\frac{1}{N} \frac{1}{\phi(1-\phi)}-2 \chi
$$

$d^{2} F / d \phi^{2}=0$ corresponds to minima in the free energy for which the curvature of the free energy is also zero, and this expression can be rearranged to obtain the locus of points called the 'spinodal line'

$$
\chi_{S}=\frac{1}{2 N} \frac{1}{\phi(1-\phi)} .
$$

Quenching a polymer blend, such that the temperature changes and the blend passes from the 1-phase region to the 2-phase region, results in 'spinodal decomposition' i.e. phase separation induced by crossing the spinodal line. This will be discussed more in section 1.6.

\subsection{Flory-Huggins-de Gennes Free Energy}

In order to study how a polymer blend undergoes phase separation, in which a 1-phase mixture de-mixes into a 2-phase mixture, we need to take into account energy costs from different phases being in contact with each other e.g. a phase rich in polymer $A$ being in contact with a phase rich in polymer $B$. The interface between these phases will have a finite width, so this interface is essentially a composition gradient across which the composition goes from $A$-rich to $B$-rich. We need to account for free energy contributions from composition gradients.

\subsubsection{Free energy of non-uniform systems}

Cahn and Hilliard $[13,14,15,16]$ are probably owed the most credit to development of theory to describe non-uniform systems. Cahn was primarily interested in binary alloys and mechanisms of phase separation and the interfaces in the resulting structures. Although the original treatment by Cahn and Hilliard was 
in the context of a binary mixture of simple fluids or quasi-solids, the theory is very general, requiring only a small change to describe polymer systems.

In the first of a series of three papers, all published under the leading title "Free energy of a non-uniform system" [13, 14, 15], Cahn and Hilliard presented "a general equation for the free energy of a system having a spatial variation in one of its intensive scalar properties" [13], which for simplicity was chosen to be a binary solution. Cahn and Hilliard's original treatment of the problem was based on expressing the local free energy $f^{*}$ "as the sum of two contributions which are functions of the local composition and the local composition derivatives" [13, 15]. For an isotropic system which has no directionality, it was then supposed that the local free energy $f^{*}$ could be expressed as

$$
f^{*}\left(c, \nabla c, \nabla^{2} c, \ldots\right)=f(c)+\kappa_{1} \nabla^{2} c+\kappa_{2}(\nabla c)^{2}+\ldots
$$

where $f$ is the energy of a uniform system, the derivatives terms represent local composition gradients and $\kappa_{i}$ are coefficients that may possibly depend on the local composition. It is noted no assumptions are made about the nature of $\kappa_{i}$, which of course could depend on local concentration [15]. The form of equation (1.35) is intuitive for an isotropic system, because only even powers of the gradient term may appear if direction is not important.

The energy $f^{*}$ refers to the local energy of a volume $d V$, hence the total free energy in a system of volume $V$ is given by

$$
F=\int_{V} f^{*} d V
$$

This result, which describes an inhomogeneous system, has two contributions to the free energy: a local contribution $f(c)$ from the system being held at composition $c$; and the energy contribution from a local composition gradient in the system. After a little re-arranging, we can express this as

$$
\begin{aligned}
F & =\int_{V} f^{*}\left(c, \nabla c, \nabla^{2} c, \ldots\right) d V, \\
& =\int_{V}\left[f(c)+\kappa(\nabla c)^{2}+\ldots\right] d V, \\
\kappa & =-d \kappa_{1} / d c+\kappa_{2} .
\end{aligned}
$$

So in general we see that $\kappa$ may indeed depend on the concentration. Equation (1.37) is limited to a regime in which the composition gradients are not too steep, or to be more exact where "the ratio of the maximum in this free energy function to the gradient energy coefficient $\kappa$ must be small relative to the square of the intermolecular distance" [13]. If this is not the case, then higher even powers of the derivatives of local concentration need to be included in equation (1.35).

Cahn and Hilliard used Eq. (1.37) to investigate the properties of the interface between two coexisting phases, and applied it to regular solutions of simple molecules [13]. The surface and interfacial energies predicted by manipulations of equation (1.37) agreed extremely well with experimental data and were in 
agreement with two empirical expressions for the latter known to generally apply. Furthermore, the theory produced extremely good agreement with data on the interfacial energy close to the critical temperature $T_{C}\left(\chi_{C}\right.$; see figure 1.3$)$, which is significant as it validated the dependence of the surface energy on the distance from the critical temperature that the theory predicted [13]. As explicitly explained by Cahn, the advantage of this representation of a non-uniform system is "the splitting of the thermodynamic quantities into their corresponding values in the absence of a gradient and an added term due to the gradient" [14].

\subsubsection{Random Phase Approximation for Polymer Chains}

The Random Phase Approximation is a self-consistent field calculation for (dense) polymer systems, attributed to de Gennes $[17,18,19,20]$. Using the RPA it is possible to find the form of $\kappa(\phi)$, the coefficient of the gradient term in equation (1.37), suitable for describing polymer systems. I will briefly follow the outline of the derivation for $\kappa(\phi)$, leaving the full derivation for the citations below.

\section{Self-consistent field calculations}

The idea behind a self-consistent field calculation (a type of mean-field treatment) for polymer systems is as follows [17]. We choose a form of interaction between polymer segments, and then derive a potential based on this interaction and the local concentration of segments. We then take an ideal/non-interacting chain and place it in this potential, and derive the resulting concentration profile. We ask if our profile for the concentration is consistent with this potential, given the interactions producing the potential, i.e., we've placed our ideal chains, now if we make them non-ideal (interacting), will the interactions between segments produce the potential? Almost certainly not, so we update the concentration profile so that it's appropriate for our potential. However, since the potential is also dependent on the concentration, we then update the potential, and then update the concentration again etc. This is an iterative procedure, and following de Gennes we can describe it as

$$
U(\mathbf{r})=T v \phi(\mathbf{r})
$$

where $T$ is temperature and $v$ is the excluded volume occupied by a segment. Given an ideal polymer chain in a potential $U(\mathbf{r})$ we can calculate a new concentration profile $\phi^{\prime}(\mathbf{r})$, and then calculate a new potential $U^{\prime}(\mathbf{r})$ etc. We hope that the potential and concentration profile converge on a stable fixed solution upon enough iterations.

De Gennes points out that the first application of a self-consistent field treatment to polymers was by Edwards [21], and I found the explanation given in Edward's work to be extremely enlightening. Edwards explains that the probability of finding a segment at distance $L$ along the chain and distance $r$ from the origin is not simply a random walk, due to the excluded volume principle a segment cannot occupy a certain volume that is excluded by the presence of another segment. Thus the probability distribution is broadened and Edwards 
shows that "it will turn out that $p$ (the probability distribution) will play the role of a potential". Note that the potential arises from the excluded volume principle, so we need only know that there is an interaction which achieves an excluded volume effect.

\section{The Random Phase Approximation}

The motivation behind the Random Phase Approximation (RPA) is: we want to compute a response function that tells us how a weak perturbation at point $r$ will effect the concentration at a point $r^{\prime}$. We will allow our chains to sit in an overall potential that is the sum of this weak perturbing potential and a self-consistent potential that is due to all of the surrounding chains. We wish to find this self-consistent potential, and this is quite a difficult problem. I will briefly describe the principles behind the random phase approximation, avoiding the dense mathematics but following the description in de Gennes book [17].

The change in local concentration at point $r$ due to a weakly perturbing potential $W\left(r^{\prime}\right)$ at point $r^{\prime}$ is

$$
\delta \Phi_{n}(\mathbf{r})=-\frac{1}{T} \sum_{\mathbf{r}^{\prime}} \sum_{m} S_{n m}\left(\mathbf{r r}^{\prime}\right) W_{m}\left(r^{\prime}\right),
$$

where the index $m$ represents segment $m$ such that $W_{m}$ is the perturbing potential acting on segment $m$, and $S_{n m}$ is a response function that relates how the perturbation on segment $m$ at $r^{\prime}$ affects segment $n$ at $r$. Thus we see that all perturbations on all segments have been included. Since we are considering an isotropic system, the response function may only depend on the separation $r-r^{\prime}$, so we switch to Fourier space to simplify the treatment

$$
\delta \Phi_{n}(\mathbf{q})=-\frac{1}{T} \sum_{m} S_{n m}(\mathbf{q}) W_{m}(q) .
$$

After some difficult maths, the central result of RPA emerges as

$$
\begin{aligned}
S_{n m}(\mathbf{q}) & =S_{n m}^{0}(\mathbf{q})-\frac{S_{n}^{0}(\mathbf{q}) S_{m}^{0}(\mathbf{q})}{\sum_{n m} S_{n m}^{0}(\mathbf{q})}, \\
& =S_{n m}^{0}(\mathbf{q})-\frac{S_{n}^{0}(\mathbf{q}) S_{m}^{0}(\mathbf{q})}{N g_{D}(\mathbf{q})},
\end{aligned}
$$

where $S_{n m}^{0}(\mathbf{q})$ is the non-interacting response function (which is known, hence allowing the substitution of the Debye scattering $g_{D}$ function for the sum over these response functions) and $S_{n}^{0}(\mathbf{q})=\sum_{m} S_{n m}^{0}(\mathbf{q})$.

What exactly does equation (1.42) mean? The derivation of this result does not involve introducing specific interactions as such, other than the implied repulsive interaction that is responsible for excluded volume, so the result really represents the distribution of polymer segments caused by there being other polymer segments around. For a detailed derivation, the reader should consult de Gennes book [17]. The main point here is that we can calculate the response function $S_{n m}$ 
from quantities that we already know. We can measure $S_{n m}$ using neutron scattering experiments, using chains partially labelled with deuterium $[19,20]$. The results of these experiments will tell us the distribution of labelled segments and therefore of the polymer chains, assuming that the labelling of segments doesn't introduce additional interactions.

\subsubsection{The Flory-Huggins-de Gennes Free Energy}

We still need to calculate a coefficient $\kappa$ of the gradient term in Eq. (1.37) suitable for polymer systems. A derivation can be found in modern textbooks $[8$, 10]. We ask how the local composition changes with respect to a change in the local chemical potential. When the volume we consider is very large compared to the chain size, such that this volume as a whole will not contain fluctuations of concentration, we obtain from equation (1.30) with $\chi=0$ (such that the polymer mixture is ideal) the chemical potential of species $i$ as $\mu_{i}=\partial \Delta F / \partial \phi_{i}$ :

$$
\mu_{i}=\frac{k T}{N_{i}} \ln \phi_{i}+\text { const }
$$

providing we write $\phi \equiv \phi_{i}$ and $1-\phi \equiv \phi_{j \neq i}$. We can then easily derive the response function that we desire

$$
\frac{\partial \phi_{i}}{\partial \mu_{i}}=\phi_{i} \frac{N_{i}}{k T}
$$

Using the notation $\delta(\Delta \mu)=\delta \mu_{A}-\delta \mu_{B}$ and noting that for a binary mixture we must have $\phi_{A}+\phi_{B}=1$, then we obtain with $\phi=\phi_{A}$

$$
\frac{\partial \phi}{\partial(\Delta \mu)}=\frac{1}{k T}\left(\frac{1}{\phi N_{A}}+\frac{1}{(1-\phi) N_{B}}\right)^{-1}
$$

This won't be correct for small volumes where fluctuations are significant. Working in Fourier space, we can adapt the latter equation to

$$
\begin{aligned}
\frac{\partial \phi(\mathbf{q})}{\partial(\Delta \mu(\mathbf{q}))} & =\frac{1}{k T}\left(\frac{1}{\phi S_{A}(\mathbf{q})}+\frac{1}{(1-\phi) S_{B}(\mathbf{q})}\right)^{-1}, \\
& =\frac{1}{k T} S_{n i}(\mathbf{q}),
\end{aligned}
$$

where $S_{n i}$ is the response function for non-interacting chains.

To account for a potential, so as to consider interacting chains, we can then write

$$
\frac{1}{S(\mathbf{q})}=\frac{1}{S_{n i}(\mathbf{q})}-V(\mathbf{q})
$$

and we note that for $q=0$ this potential must equal $2 \chi$, since by definition this is our interaction in the $\mathrm{FH}$ regime based solely upon the enthalpy between two monomers. For small $q$ it must be true that

$$
V(\mathbf{q})=2 \chi\left(1-\frac{1}{6} q^{2} r_{0}^{2}\right)
$$


because this term arises from a first order expansion of a Gaussian distribution describing a bare response function for a non-interacting chain $[17,20]$ and $r_{0}$, which is of the order of the segment size $a$ (which is therefore equal to the lattice spacing in the Flory-Huggins lattice), measures the range of inter-segment forces [8]. Inserting the approximate potential $V$ and the response functions $S_{A}$ and $S_{B}$ into equation (1.47) we obtain an expression for the scattering response function $S(\mathbf{q})$ that is consistent with a free energy (in units of $k_{B} t$ ) of the form [8]

$$
\begin{gathered}
F=\int\left[f_{F H}(\phi)+\kappa(\phi)(\nabla \phi)^{2}\right] d \mathbf{r}, \\
\kappa(\phi)=\frac{\chi r_{0}^{2}}{6}+\frac{a^{2}}{36 \phi(1-\phi)} \approx \frac{a^{2}}{36 \phi(1-\phi)},
\end{gathered}
$$

for which it is common practice to neglect the small first term in $\kappa(\phi)$. The result is the Flory-Huggins-de Gennes free energy for a binary polymer system:

$$
F[\phi, \nabla \phi]=\int\left[f_{F H}(\phi)+\frac{a^{2}}{36 \phi(1-\phi)}(\nabla \phi)^{2}\right] d \mathbf{r}
$$

which is the starting point for studying the kinetics of, and morphology resulting from, phase separation of polymer blends.

\subsection{Spinodal Decomposition}

A mixture of two components may exist either as one phase (the entropy of mixing overcomes the heat of mixing) or as two phases (the heat of mixing overcomes the entropy of mixing). A phase diagram like figure 1.3 separates regions of stability of blends existing as one-phase and two-phases. Phase separation from one phase into two phases, caused by the thermodynamic instability of the mixture as it is brought across the spinodal line from the one-phase to the two phase region, is called Spinodal Decomposition. I will first discuss an early example involving a crystaline solid, not only because it is an important example in the development of theory, but because it is a good introduction to several concepts.

\subsubsection{A crystal with a 1D inhomogeneity}

Hillert considered a crystalline solid consisting of two components $A$ and $B$, in which a variation in composition $x$ (the volume fraction of $A, 0<x<1$ ) was allowed in one direction along the crystal [22]. This system was modelled by consecutive parallel $2 \mathrm{D}$ planes $i-1, i, i+1 \ldots$, every plane having some characteristic composition $x_{i-1}, x_{i}, x_{i+1} \ldots$. Figure 1.4 shows a schematic representation.

Hillert calculated the free energy of this system. For the interaction energy (heat/enthalpy), it was assumed that an atom in a particular plane $i$ could interact with $Z$ nearest neighbours in total, with $z$ of these nearest neighbours being located in the next plane $i+1$. The system as a whole has average composition $x_{a}$, interaction strength $v$, and the total number of atoms within a single atomic plane 


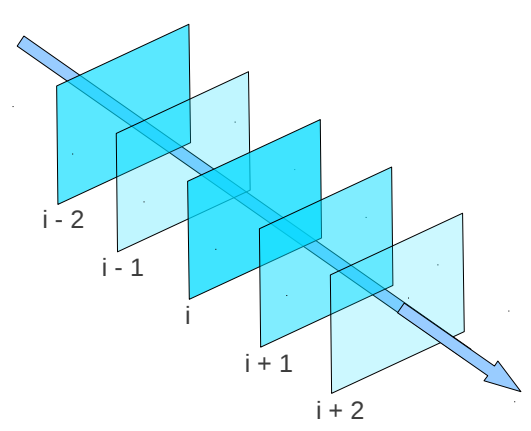

Figure 1.4: Parallel 2D planes of a crystal, in which the composition of each plane $0<x_{i}<1$ is represented, in this schematic, by the degree of transparency of the planes. The arrow represents the direction of inhomogeneity in the crystal.

is $m$. The energy of interaction for plane $i$ interacting with next plane $i+1$ is then $\Delta U=v m\left\{Z\left(x_{i}-x_{a}\right)^{2}-z\left(x_{i}-x_{i+1}\right)^{2}\right\}$. The change in entropy arising from a single plane $i$ being at a composition different from the average composition is given by regular solution theory $\Delta S=m\left\{x_{p} \log \frac{x_{p}}{x_{a}}+\left(1-x_{p}\right) \log \frac{1-x_{p}}{1-x_{a}}\right\}$. Hence, after summing across all planes in the system, the energy difference between the inhomogeneous state and the homogeneous (note the direction of consideration of energy difference, which gives a minus sign) is

$$
\begin{aligned}
\Delta F= & -v m \sum_{p}\left\{Z\left(x_{p}-x_{a}\right)^{2}-z\left(x_{p}-x_{p+1}\right)^{2}\right\} \\
& +k_{B} T m \sum_{p}\left\{x_{p} \log \frac{x_{p}}{x_{a}}+\left(1-x_{p}\right) \log \frac{1-x_{p}}{1-x_{a}}\right\} .
\end{aligned}
$$

\section{Nature of stable solutions}

Hillert considered stable (mathematical) solutions to the problem, which requires calculation of the change in free energy "when atoms are exchanged between two neighbouring planes $p-1$ and $p$ " i.e. what is the functional derivative of the free energy with respect to composition $x_{p}$ of plane $p$. For equilibrium (stable solutions) we require $\delta \Delta F / \delta x_{p}=0$. For small amplitude fluctuations around the average composition $x_{a}$, stable solutions were found to obey the relation

$$
x_{p+1}=x_{p-2}-x_{p-1}+x_{p}-2 M\left(x_{p-1}-x_{p}\right),
$$

where $M$ is a constant given by a combination of parameters (including average composition $x_{a}$, the number of nearest neighbours $Z$ and $z$, the temperature $T$, and the interaction energy $v$ ).

It turns out that $M=1$ corresponds to the spinodal curve for a $1 \mathrm{D}$ system: (one-phase region) $|M|>1$ corresponds to states outside the spinodal for which the only physically relevant solution (in which $0<x_{p}<1$ ) was $x_{p}=$ const $=x_{a}$ i.e. a homogeneous state; (two-phase region) $|M|<1$ corresponds to inside the 
spinodal, for which relevant solution for small amplitude fluctuations are of the form $x_{p}=x_{a}+C \sin p \phi$ where $C$ is a constant. For shallow depths beyond the spinodal, the wavelength (of the composition variation) extends over many atomic planes, but as distance into the spinodal increases $(|M| \rightarrow 0)$ the wavelength becomes of order unity (on the order of a few atomic planes). Consideration of large compositional variations required numerics to be performed on a computer, but the results showed that again the equilibrium states within the spinodal were sinusoidal in nature.

\section{Wavelengths}

Hillert supposed that a kinetic treatment of the problem would give insight into what composition variation wavelengths might dominate by showing which wavelengths would grow the fastest. It was also noted that in order for the system to increase the wavelength of fluctuations (in order to lower energy) a re-arrangement of the system is necessary that should also be studied from a kinetic perspective. By deriving a diffusion equation for the system and applying random fluctuations (fluctuations with a spectrum of amplitudes and wavelengths), Hillert found that a spectrum of wavelengths first developed, followed by small wavelength fluctuations decreasing in amplitude, causing the average wavelength of the system grow with time. Consideration of the fastest growing wavelength is important in spinodal decomposition studies [22].

\subsubsection{Stability of a solution}

Cahn considered the stability of a solid-solution with respect to compositional fluctuations [23], where 'solution' is meant in the sense of a binary mixture which may support composition gradients, and 'solid' is meant in the sense that there is an elastic energy contribution to the free energy (arising from strain in the material when an initially homogeneous region becomes inhomogeneous). I will leave out the elastic energy contribution in my discussion here.

Cahn considered the free energy of a two-component solution using Eq. (1.37) To consider fluctuations requires knowledge of how the free energy changes when a small amount of one-component is replaced with another, but "in the presence of a gradient, if we make a local change in composition we also change the local gradient", so we must consider the functional derivative of the free energy with respect to composition. If a functional $F$ is given by

$$
F=\int g(r, c(r), \nabla c(r)) d V
$$

then the functional derivative of $F$ with respect to $c(r)$ is given by

$$
\frac{\delta F}{\delta c}=\frac{\partial g}{\partial c}-\nabla \cdot \frac{\partial g}{\partial(\nabla c)}
$$


as long as the integrand vanishes at the boundaries of integration. Applied to Eq. (1.37) (such that $g$ is the integrand $f^{*}$ of Eq. (1.37)) we obtain

$$
\frac{\delta F}{\delta c}=\frac{\partial f}{\partial c}+\frac{\partial \kappa}{\partial c}(\nabla c)^{2}-2 \kappa \nabla^{2} c
$$

The functional derivative can be used to formulate a diffusion equation which may be used to study the morphology resulting from spinodal decomposition.

\subsubsection{Diffusion Equation}

The chemical potential $\mu$ can be related to the functional derivative via $\mu=$ $\delta F / \delta c$. Cahn considered the matter current $J=-M \nabla \mu$, where $M$ is a positive mobility coefficient, and the continuity equation $\partial c / \partial t=-\nabla \cdot J$. Disregarding all terms non-linear in $c$, so as to consider infinitesimal compositional fluctuations corresponding to the initial stages of spinodal decomposition, we have

$$
\frac{\partial c}{\partial t}=M \frac{\partial^{2} f}{\partial c^{2}} \nabla^{2} c-2 M \kappa \nabla^{4} c
$$

confirming Cahn's assertion that "the diffusion equation must contain a higher order term reflecting the thermodynamic contributions of the gradient energy term". The first term of Eq. (1.57) allows us to interpret $M f^{\prime \prime}$ as an interdiffusion coefficient. The second term accounts for gradients and interfaces.

\section{Wavelengths}

For small variations in $c$ about the average $c_{0}$, the solution to Eq. (1.57) is $c-c_{0}=A(k, t) \cos k \cdot r$, where $k$ is the wavevector of a compositional variation and $A(k, t)$ is an amplification factor depending on the wavelength, which yields

$$
\frac{\partial A}{\partial t}=-M k^{2}\left[\frac{\partial^{2} f}{\partial c^{2}}+2 k^{2} \kappa\right] A
$$

and therefore solutions are of the form

$$
\begin{aligned}
& A(k, t)=A(k, 0) \exp [R(k) t], \\
& R(k)=-M k^{2}\left[\frac{\partial^{2} f}{\partial c^{2}}+2 k^{2} \kappa\right],
\end{aligned}
$$

Cahn referred to $R(k)$ as a kinetic amplification factor, which if negative means that the solution is stable to fluctuations of wavevector $k$, and which if positive means the the solution is unstable to fluctuations of wavevector $k$. The critical wavelength by definition separates these two regimes, and corresponds to the smallest possible wavelength for which the mixture is unstable, $R\left(k_{c}\right)=0$. Cahn noted that "surface tension prevents decomposition of the solution on too fine a scale." This important point is why equations like (1.37) and (1.51) are required to study phase separation, because without a gradient energy term, the mixture could decompose on an infinitely fine scale. However, since this would yield an 
enormous amount of gradient energy, this is not actually energetically favourable, and so does not happen.

Cahn found that the fastest growing wavelength was related to the critical wavelength

$$
k_{\text {max }}=\sqrt{2} k_{c},
$$

Fluctuations of wavelength $k_{\max }$ "will grow the fastest and will dominate. This principle of selective amplification depends on the initial presence of these wavelengths but does not critically depends on their exact amplitude relative to other wavelengths". This is a very important idea in spinodal decomposition.

\subsubsection{Morphology from spinodal decomposition}

To investigate the structures that may result from spinodal decomposition, Cahn used the solution to Eq. (1.57) given by $c-c_{0}=A(k, t) \cos k \cdot r$ [16]. Since all sums of all solutions are also possible solutions, due to superposition theory, the most general solution is

$$
c-c_{0}=\sum_{\text {all } \mathrm{k}} \exp \{R(k) t\}[A(k) \cos (k \cdot r)+B(k) \sin (k \cdot r)] .
$$

The problem of studying the temporal evolution is much simpler if only the wavelength with the fastest growing amplitude is considered i.e. $k_{\max }$

$$
c-c_{0} \approx \exp \left\{R\left(k_{\max }\right) t\right\} \sum_{k_{\max }}[A(k) \cos (k \cdot r)+B(k) \sin (k \cdot r)] .
$$

Hence "The predicted structure may be described in terms of a superpositioning of sinusoidal composition modulations of a fixed wavelength, but random in amplitude, orientation, and phase" and "at some time after phase separation starts, a description of the composition in the solution will be a superposition of sine waves of fixed wavelength, but random in orientation, phase, and amplitude". The sum in equation (1.63) remains, even though only $k=k_{\max }$ is considered in the sum, because Cahn generated a predicted morphology by summing over waves with different directions and amplitudes.

The resulting morphology was a highly interconnected bi-continuous structure, which resembled that of phase separable glasses believed to have undergone spinodal decomposition. Cahn stated that "theory of spinodal decomposition has been shown to predict a two-phase structure", although strictly speaking this result only applied to the initial stages of phase separation. Kinetic restrictions would of course mean that this structure would indicate the qualitative features that would be expected from the late stages, since rearrangement of material at late stages is restricted by the structures formed at early stages. Figure 1.5 shows simulation snapshots of a phase separating polymer-blend, produced by solving the diffusion equation (1.65) for a polymer-blend (equation (1.65) is essentially equation (1.57), but with random thermal noise included and without limiting to small variations around $c_{0}$ ) shown for visualisation purposes: the final morphology, a bicontinuous structure, is very similar to that obtained by Cahn. 

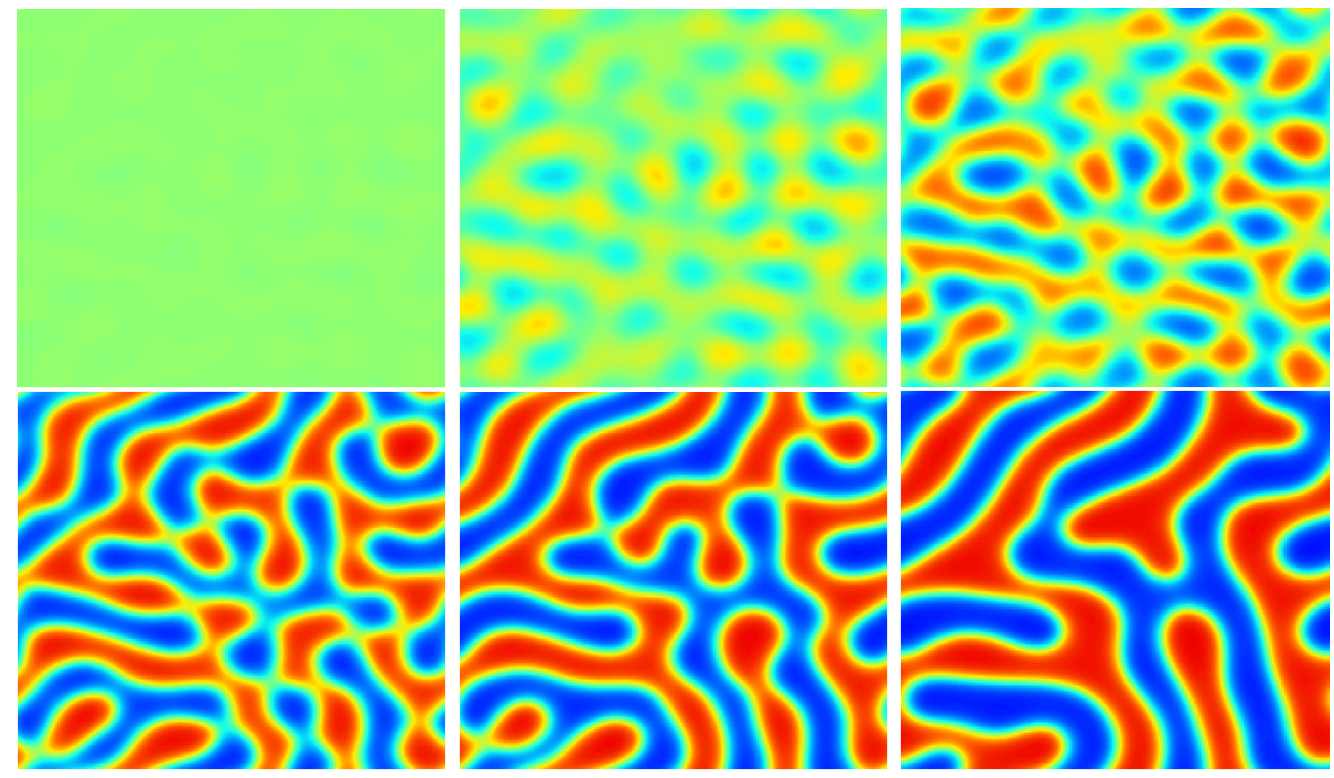

Figure 1.5: Shown here only for visualisation purposes are simulation snapshots I produced of a phase separating symmetric polymer blend $\left(N_{A}=N_{B}=N\right.$, average volume fraction $\bar{\phi}=1 / 2$ ), created by solving the Cahn-Hilliard-Cook equation (1.65) for a polymer blend. The initially nearly-homogeneous blend phase separates and coarsens into a highly interconnected bicontinuous morphology, the latter of which is similar to that obtained by Cahn.

\subsubsection{Random noise and spinodal decomposition}

Cahn's theory of the early stages of spinodal decomposition [16] is known to break down at later stages, mainly as a result of neglecting higher order terms in the gradient energy that bring in other harmonics [24]. However, Cook noted that it was not understood why the theory could also break down for the initial stages of spinodal decomposition for which it was designed to study. Cook suggested that "the breakdown at the very early stages of the transformation which is caused by thermal fluctuations is not so widely appreciated" [24]. A strong example of the lack of understanding was the complete lack of spinodal decomposition in some glass mixtures, which were practically identical to other glass mixtures which did have the features of spinodal decomposition. This could not be accounted for by a theory that suggested that only the initial amount of decomposition in the glass mixture (the spectra of composition fluctuations in the initial mixture) would result in different late-time features.

Supposing that "fluctuations in composition caused by thermal effects which were not included in the original theory" may have been responsible, Cook modified Cahn's diffusion equation given in Eq. (1.57) to include thermal noise, which should give rise to Brownian motion of the fluid. This was justified by Cook because it is understood that "the equilibrium state is dynamic and that, for the case of a stable, single phase, binary solid solution, an appreciable flux of solute occurs at equilibrium." To include this random thermal contribution, Cook modified the matter current equation $J=-M \nabla \mu$ to include a "quasi-random thermal 
contribution to the total flux", denoted by $j$, resulting in a material current

$$
\begin{aligned}
J & =-M \nabla \mu+j \\
& =-M \nabla \frac{\delta F}{\delta c}+j .
\end{aligned}
$$

Cook's important contribution to the rate equation for spinodal decomposition lead to the name "Cahn-Hilliard-Cook" theory for equations of the form

$$
\begin{aligned}
\frac{\partial c}{\partial t} & =\nabla \cdot\left[M \nabla \frac{\delta F}{\delta c}\right]-\nabla \cdot j(r, t) \\
& \equiv \nabla \cdot\left[M \nabla \frac{\delta F}{\delta c}\right]+\eta(r, t) .
\end{aligned}
$$

The randomly fluctuating field $\eta(r, t)$ has certain properties, such that its average value is zero. Using averaging to treat the random term (the average properties are well defined), the rate of change equation given by Cahn in equation (1.58) gains an extra term, giving $d I(k, t) / d t=M(k)\left\{\left[f^{\prime \prime}+2 \kappa k^{2}\right] I(k, t)-\right.$ $\left.k_{B} T / \Omega c_{0}\left(1-c_{0}\right)\right\}$, where $\Omega$ is the volume per atom. So the rate of change of intensity has two separate contributions: (a) a thermodynamic driving force "which is proportional to the free energy associated with the Fourier coefficient of wavevector k"; and (b) a thermal driving force "which is proportional to the temperature and independent of the wave vector".

The inclusion of thermal noise has several non-trivial implications for spinodal decomposition [24], especially in the early stages of spinodal decomposition when the free energy of the fluctuations is $\sim k_{B} T$ "and thus the influence of random fluctuations will be pronounced": (i) the critical wavevector $k_{C}$ is now determined by the condition that the thermal driving force (from the thermal noise) is equal to the thermodynamic driving force (arising from the free energy of the system); (ii) the rate of intensity will be greater given the thermal driving force, since "every movement in the fluctuation field... which increase the magnitude of a Fourier coefficient is amplified"; and (iii) the "thermal driving force indicates early stages of decomposition outside the spinodal... in this 'operational' sense the spinodal, itself, becomes a diffuse boundary".

\subsubsection{Spinodal Decomposition of a Polymer Blend}

I will briefly discuss, for completeness, the relaxation of a polymer melt, which is an important idea of spinodal decomposition, although the concept will not be discussed in the rest of this thesis. Relaxation concerns, to give a broad definition, how an unstable mixture 'relaxes' into a stable mixture in spinodal decomposition. Relaxation can be described by a relaxation time for different wavelengths (lengthscales) of the decomposition.

De Gennes extended the study of the dynamics of spinodal decomposition to polymer blends [18]. For polymer blends, there are a variety of length scales that are important, and so it may be important to have a dependence of the mobility on the wavelength of fluctuations in a polymer blend. This can be 
done by introducing a wavelength dependent Onsager coefficient $\Lambda(q)$ into the usual expression for the matter current $J=-M \nabla \mu$. This effectively allows a dependence of the constant $M$ on the wavelength of each Fourier component. The result is a current for each Fourier component

$$
J_{q}=-\frac{\Lambda(q)}{k_{B} T}(\nabla \mu)_{q}
$$

We can allow use the following expression for the relaxation time for a mode of wavelength $q$ :

$$
\frac{1}{\tau_{q}}=-\frac{1}{\delta \phi_{q}} \frac{\partial\left(\delta \phi_{q}\right)}{\partial t}
$$

where $\delta \phi$ is a small fluctuation away from the homogeneous state $\phi_{0}$, such that we can express the composition using $\phi=\phi_{0}+\delta \phi$. If wavelengths of fluctuations produce negative values for $\tau_{q}^{-1}$, then compositional fluctuations of this wavelength grow with time.

De Gennes derived a relaxation formula for a symmetric binary polymer blend, assuming the form $\Lambda(q) \propto q^{2}$ for polymer blends (based on a scaling ansatz) [18]. The result for the relaxation time "differs from the standard Cahn-Hilliard equation for spinodal decomposition" for simple molecules, this difference arising from "the presence of long chains". It was noted that "the characteristic length $l$ is much smaller than the coil size... (thus) spinodal decomposition is an excellent probe for fluctuations of short wavelength." The assumption $\Lambda(q) \propto q^{2}$ was later found to be false [25].

Pincus continued the work of de Gennes by taking into account new knowledge the nature of the Onsager coefficient [25]. The nature of the relaxation of modes in a polymer melt leads to a significantly altered dependence of the Onsagar coefficient on the wave vector $q$, namely that $\Lambda(q) \propto q^{-2}$. This gives a very different result for the relaxation time (still of the form equation (1.68)). Unlike the results of the earlier work by de Gennes which showed that spinodal decomposition should probe very short wavelengths "much smaller than the coil size" [18], it now appeared that "the unstable mode has a wavelength comparable to the ideal chain radius and therefore should vary as $N^{1 / 2}$ with only a weak concentration dependence". Also, "the corresponding growth rate is proportional to the reptation diffusion coefficient in a melt and thus scales as $N^{-2}$ and has a concentration dependence that reflects the shape of the spinodal line." Concerning the latter point, this means that upon going from the one-phase to the two-phase region, the rate of spinodal decomposition depends on the concentration.

\section{Mean-field treatments of polymer systems}

Binder later did a similar calculation, but using the chemical potential as calculated via functional derivatives [26]. Binder notes that mean-field treatments of spinodal decomposition in fluids of simple molecules can fail due to fluctuation effects that are not included in mean-field treatments. However, "a simplifying feature due to the large size of the polymer chains is the mean-field character of the unmixing transition, fluctuation corrections to the mean-field description can be 
safely neglected." On the linearisation approximation $\phi(r, t) \equiv \phi_{0}+\delta \phi(r, t)$ used to calculate the relaxation time, Binder noted that "whilst it is well known that the linearisation approximation is not valid in the critical region of non-mean-field liquid... its validity in the present case should be much better justified." The main result is that "the wavevector $q_{m}$ of maximal growth in spinodal decomposition is typically of the order of $q_{m} \sim R^{1}$ " where $R$ is the polymer coil radius.

\subsection{Summary}

In this chapter, I discussed the development of theory to describe bulk polymer blend systems, beginning with the development of an entropy of mixing valid for long chain molecules, followed by a heat of mixing, and an expression for the free energy cost of compositional gradients. Together, these expressions give the Flory-Huggins-de Gennes free energy of mixing. I discussed the coexistence curve and spinodal line for a binary blend system, as well as spinodal decomposition whereby a blend phase separates into phases rich in either component upon being quenched from the one-phase to the two-phase region. This chapter has covered the bulk theory required in this thesis, and the next chapter extends this theory to include surfaces, allowing films to be studied theoretically. 


\section{DeVElopment OF THEORY FOR POLYMER-Blend Thin Films}

I follow the development of theory for thin films of binary mixtures from a chronological perspective. Beginning with the inclusion of a surface into non-uniform systems, I discuss the origin of the form of surface energy used in this thesis, cover the concept of wetting of surfaces by a preferred phase, and discuss how phase separation in films is affected by preferential attraction of components by surfaces and by finite confinement effects.

2.1 Introduction . . . . . . . . . . . . . . . . . . . 28

2.2 Non-uniform systems with a surface . . . . . . . . . . . . . . . . . . 29

2.3 Form of the surface energy . . . . . . . . . . . . . . . . . . . . . . . . . . . . . . 30

2.4 Wetting in semi-infinite geometries . . . . . . . . . . . . 34

2.5 Phase Separation in finite geometries . . . . . . . . . . . . . 37

2.6 Summary . . . . . . . . . . . . . . . . . . . . . 44 


\subsection{Introduction}

The aim of this chapter is to introduce several important concepts about films of binary fluids that are relevant to the rest of this thesis. I have taken care to follow the primary literature that developed the relevant theory, much of which is as relevant to simple fluids and Ising systems as it is to polymer fluids. This chapter can be summarised as follows. I begin with how a surface can be included into a theory of non-uniform systems, and follow literature for Ising systems, simple fluid systems, and polymer systems which utilised specific forms of this surface energy. I then briefly discuss wetting, whereby a surface can be coated by the preferred phase of a binary mixture. I then introduce films of multicomponent fluids, namely a binary mixture bounded by two surfaces, and explain different surface energy configurations caused by the preferential attraction of components by the two surfaces, and what effect the surface energy and finite geometry has on phase separation.

It is useful to introduce some terminology here. Figure 2.1 shows two schematics of semi-infinite systems: (a) bulk system of infinite extent in contact with a surface/ wall $^{7}$ (such that the system spans from $z=0$ to $z=\infty$, where $z$ measures the distance from a bounding wall at $z=0$ ); and (b) a film of infinite thickness, which is effectively a film of thickness $d$ in the limit that $d \rightarrow \infty$ (usually such that the system runs from $z=-\infty$ to $+\infty$, the position of the two bounding surfaces/walls). The work that I discuss prior to section 2.5 considers such semi- $\infty$ geometries.

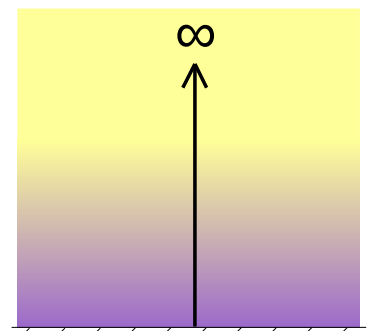

(a)

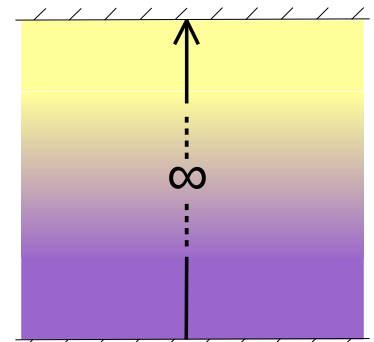

(b)

Figure 2.1: Two different examples of semi-infinite systems of two-phase mixtures in contact with surfaces, the degree of shading (colouring) representing composition: (a) bulk system of infinite extent in contact with a single surface/wall/substrate; (b) a film of infinite thickness with two bounding surfaces.

\footnotetext{
${ }^{7}$ Surface/Wall: the boundary formed by the interface between the fluid and, for example, air or a vacuum. While the terms will often be used interchangeably, a Wall is specifically meant to be a rigid planar surface, while a Surface could be non-rigid and deformable. A substrate such as a silicon wafer, on which a fluid film may rest, is therefore a wall, whereas the fluid-air boundary may be referred to as either a wall or a surface depending on the context.
} 


\subsection{Non-uniform systems with a surface}

In pioneering work, Cahn extended the theory for non-uniform bulk systems, with two phases $\alpha$ and $\beta$, to include a third phase $x$ representing a surface [27]. Later work concerning similar systems is almost invariably built on these foundations. Cahn showed that for a two-phase system $\alpha$ - $\beta$ in contact with a third phase $x$, near criticality for the two-phase system the third phase is completely wetted by only one of the critical phases, the other critical phase being entirely excluded from contact with the third phase [27]. Figure 2.2 is a schematic similar to that given in Cahn's work: when the angle that a phase makes with the surface drops to zero, $\theta \rightarrow 0$, the surface is wetted by that phase. Applied to a binary fluid (twophase system) in contact with a wall (the third phase), which was the context of the work, this means that near criticality the wall would be wet entirely by a phase rich in one component of the binary fluid.

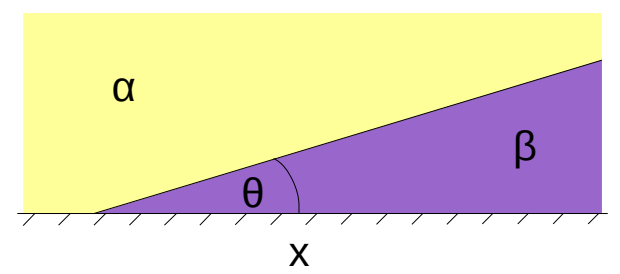

Figure 2.2: Schematic of a two-phase system $\alpha$ - $\beta$ in contact with a third phase $x$, which is in this case a planar surface. When $\theta \rightarrow 0$, the surface will be completely wetted by the $\beta$ phase, such that a layer of $\beta$ phase will coat the surface, excluding the $\alpha$ phase from contact with the surface. Here, the surface is non-wet since $\theta \neq 0$, but changing parameters like temperature and surface energy could cause $\theta \rightarrow 0$.

Cahn modelled a semi-infinite system, running from $z=0$ to $z=\infty$ as shown in figure 2.1(a), of a binary blend of liquid-vapour in contact with a surface. The bulk composition at an infinite distance from the surface at $z=0$ was $c_{\infty} \equiv c_{0}$. Cahn assumed that "the interactions between surface and fluid are sufficiently short-range" and only depend on the local fluid composition $c_{s}$ at the surface. The excess free energy the system has, due to the presence of the wall, can then be expressed as

$$
\Delta F=\Phi\left(c_{s}\right)+\int_{0}^{\infty} \Delta f+\kappa\left(\frac{d c}{d x}\right)^{2} d x
$$

where $\Delta f=f(c)-f\left(c_{0}\right)-\left.\left(c-c_{0}\right)(\partial f / \partial c)\right|_{c=c_{0}}$ is the energy needed to form a volume of material with composition $c$ differing from the bulk $c_{0}, \kappa(d c / d x)^{2}$ is the energy cost of a composition gradient, and $\Phi\left(c_{s}\right)$ is the surface energy. The surface energy decreased non-linearly with increasing composition of the preferred component at the wall, such that coating of the wall by that component would be preferable. Cahn made no other assumptions about the form of $\Phi$.

The task of finding the equilibrium profile $c(z)$, describing the composition $c$ with distance $z$ from the wall, requires minimising $\Delta F$ with respect to a boundary condition imposed by the wall. Setting the functional derivative of the excess free 
energy at the wall to zero, Cahn obtained for the boundary condition at $z=0$ :

$$
\frac{d \Phi}{d c_{s}}-\left.2 \kappa \frac{d c}{d x}\right|_{c=c_{s}}=0 .
$$

(Calculating equilibrium profiles is the subject of chapter 3.) I will discuss the important results of Cahn's work, as opposed to only the model, in section 2.4, but equations (2.1) and (2.2) are ideal to introduce the concept of the surface energy addition to the bulk free energy.

\subsection{Form of the surface energy}

In this section, I will discuss the form of the surface energy term $\Phi$ in equation (2.2), for which there are many applications to different non-uniform systems, including Ising-spin systems (magnetic systems), multicomponent fluids of simple molecules, and polymer blends. It turns out the the form of the surface energy is essentially the same in all these cases, although the meaning of the terms varies slightly between each case.

\subsubsection{Magnetic systems}

Lubensky and Rubin studied a semi-infinite system of continuous magnetic spins using Landau-Ginzberg mean-field theory [28]. Figure 2.3 is a schematic, showing a wall (surface), the layer of spins directly adjacent to the wall, and the next layer of spins (similar to figure 2.1(a), these spins extended infinitely away from the wall). The effect of the wall had two contributions: a surface magnetic field at that wall (an interaction between the wall and the spins, which would encourage alignment of the spins with the magnetic field at the wall) and an enhancement of the nearest neighbour exchange energy at the wall (which would encourage the spins directly adjacent to the wall to align with each other either more strongly or less strongly than they would in the bulk). In effect, the enhancement of nearest neighbour exchange energy represents a change of the nature of interactions between spins at the wall, which should be possible even if the wall does not exert a surface magnetic field on the spins.

The presence of the wall resulted in a boundary condition determining the magnetisation at the wall, which is in analogy with the boundary condition equation (2.2). The surface highly perturbs the behaviour of the system. For example, when the surface field exceeds the bulk field, then surface transitions are possible in which the surface orders when the bulk is still disordered (the magnetisation decaying with distance from the wall). Due to the enhancement of nearest neighbour interactions, it is also possible for the bulk to order before the surface orders.

Pandit and Wortis (1982) also studied a semi- $\infty$ Ising system like that in figure 2.3, but with a one-dimensional inhomogeneity allowing the spin-profile to vary with distance from the wall [29]. They used a phase portrait method (phase portraits are the topic of chapter 3) to study the states and transitions of the 


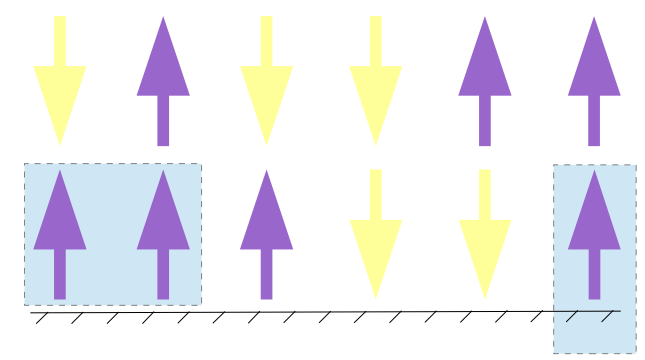

Figure 2.3: Two layers of a semi- $\infty$ Ising system in contact with a wall, which can modify the interactions between neighbouring spins in the vicinity of the wall (left) and apply a magnetic field to spins adjacent to the wall (right).

system. Similar to Lubensky and Rubin [28], both a surface field and a surface enhancement were used to represent the wall: the surface term was linear in the magnetisation at the wall $M(0)$; the surface enhancement, corresponding to an additional interaction between spins in the immediate vicinity of the surface, provided a term quadratic in $M(0)$. The particular form is noted here for comparison with that used for simple fluid and polymer systems, discussed in sections 2.3.2 and 2.3.3. Variation of the free energy functional at the surface leads to a boundary condition analogous to that found by Cahn, equation (2.2).

\subsubsection{Simple Fluid systems}

The form of the wall interaction used by Lubensky and Rubin [28], briefly mentioned above, turns out to be qualitatively identical to that which became the norm for binary fluid systems. Nakanishi and Fisher studied the "global phase diagram for wall and surface critical phenomena" [30]. Their work was a direct extension of that by Lubensky and Rubin, although leaning towards the context of binary fluid systems in contact with a surface. The equivalence of the surface field in magnetic systems and an "incremental chemical potential, $\delta \Delta \mu=h_{1} k_{B} T$, which favours one species and acts only near the wall" was made clear, and as in the case of an Ising system, a surface enhancement $g$ representing enhanced coupling (a change in the nature of interactions) near the wall was included. Figure 2.4 is a schematic representing these effects. The surface energy contribution, depending on the magnetisation at the surface $m_{1}$, was then

$$
f_{s}\left(m_{1}\right)=-h_{1} m_{1}-\frac{1}{2} g m_{1}^{2}
$$

which is essentially equivalent to that used by Lubensky and Rubin for an Ising system [28]. Using $\Phi=f_{s}$ and $c_{s}=m_{1}$ in equation (2.2), it is simple to see that the boundary condition at the surface will be of the form $\partial_{z} m_{1} \propto h_{1}+g m_{1}$. Hence the surface enhancement $g$ sets the boundary condition on $m_{1}$ by relating the composition gradient to the surface composition. Perhaps they were first to write the surface energy in a phenomenological form like in equation (2.3).

Using the same description of a simple bulk fluid in contact with a surface, Nakanishi and Pincus later studied 'surface spinodal decomposition' (where the 


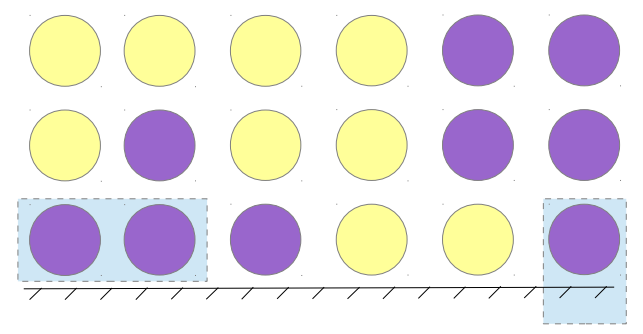

Figure 2.4: Three layers of a semi- $\infty$ binary fluid of simple molecules in contact with a wall, which can modify the interactions between neighbouring molecules in the vicinity of the wall (left) and apply an incremental chemical potential favouring one component on molecules adjacent to the wall (right).

surface gives directionality to spinodal decomposition) for fluids near a wall [31], describing the model as "the simplest model for wetting where an incremental chemical potential $\delta \Delta \mu=h_{1} k_{B} T$ is introduced favouring one component, say $B$, of a binary fluid mixture near the wall" with an enhancement term describing an "enhancement near the wall in the effective molecular couplings".

Jerry and Neumann investigated the criteria for depletion and enrichment of a component of a binary mixture at a wall, using a nearest-neighbour type interaction to model interaction with the wall [32]. Using regular solution theory for the binary mixture, they pointed out that a consistent form for the wall interaction energy $\Phi$ must be (at least) quadratic in the local composition $c_{s}$; the coefficient of the linear term is a chemical potential and the coefficient of the quadratic term is a based on missing neighbour interactions caused by the presence of the wall resulting in fewer nearest neighbour bonds at that wall. Of course, such a surface energy is then equivalent to equation (2.3). They showed the equivalence of various theoretical descriptions of a wall, including a 'frozen wall' consisting of fluid molecules with a fixed phase $c_{s}$, a free surface in which the interface can be taken to be a vacuum, and a 'real wall' which included the effects of the wall interaction with each component in the binary mixture. A function quadratic in composition proves to describe all of these scenarios, only the coefficients have different physical meanings, the proposed form being $(s \equiv c)$

$$
\Phi(s)=-\mu_{1} s-\frac{1}{2} g s^{2}+R,
$$

where $R$ is a constant, which would of course disappear in the boundary condition (2.2). One of the most important points they raised was that "the bulk concentration is important in determining whether enrichment or depletion occurs (at the wall). This contradicts the conventional understanding that the surface should be enriched with the component having the lower surface energy" [32].

\subsubsection{Polymer systems}

Schmidt and Binder extended the work of Nakanshi and Pincus to binary polymer blends in the presence of a single wall [33]. Their results showed that polymer systems should display the same surface phenomena present in simple fluid systems. 
They used a quadratic expression for the bare surface energy $-\mu_{1} \phi_{1}-\frac{1}{2} g \phi_{1}^{2}$, in common with the aforementioned work on systems of simple molecules. Similarly, $g$ "represents a change of interactions near the surface (including the effects due to "missing neighbours" etc.)." Using the Flory-Huggins-de Gennes free energy functional, together with a chemical potential difference and the surface energy, they wrote their free energy functional in the same form as equation (2.1):

$$
\frac{F}{A k_{B} T}=\int_{0}^{\infty}\left\{f_{F H}(\phi)+\kappa(\phi)\left(\frac{d \phi}{d z}\right)^{2}-\Delta \mu \phi\right\} d z-\mu_{1} \phi_{1}-\frac{1}{2} g \phi_{1}^{2} .
$$

Essentially, the interactions between polymer segments and a wall should be similar to interactions between simple molecules and a wall. Figure 2.5 is a schematic showing why this ought to be the case. They put forth that, unlike for simple fluids in which fluctuations can make artefacts of mean-field predictions, "polymer mixtures would be an excellent candidate of systems to observe (phenomena like) critical wetting" which are predicted by such theories, since polymer mixtures should be better described by mean-field theories.

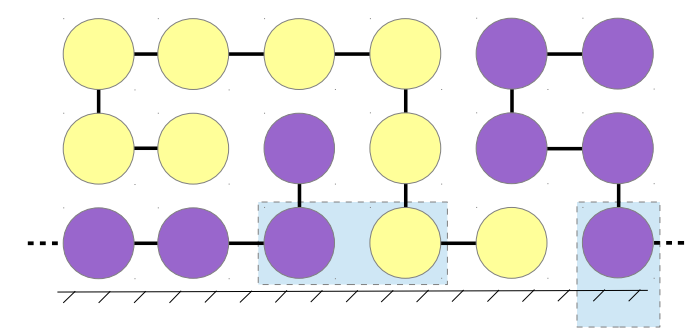

Figure 2.5: Schematic of polymer chains in the vicinity of a surface/wall. The presence of the wall can modify the interactions between neighbouring segments on different chains (centre), whether these chains are of the same species or of a different species, and apply an incremental chemical potential near the wall which favours segments of one polymer species (right). The interactions between different monomers, and between monomers and the wall, ought to be of the same nature and magnitude as the interactions of simple molecules.

\section{Interpretation of the quadratic wall interaction for a polymer system}

Although a quadratic expression for the free energy of the boundary is mainly phenomenological, one can make a simple argument based on bond counting that shows that a quadratic dependence is fairly general. Jones proceeds as follows [8]: Consider a binary polymer mixture $A-B$ laid out on a lattice. The probability of a particular lattice site being occupied by a segment of $A$ is $\phi$, which is the total volume fraction of $A$ in the blend. If the bond energy between components $i$ and $j$ on neighbouring lattice sites is $\epsilon_{i j}$, then the energy contribution from $A$ monomers in contact with $A$ monomers will be the probability of a site being occupied by $A$, which is $\phi$, multiplied by the probability that a neighbouring site is also occupied by $\mathrm{A}$, which is also $\phi$, multiplied by $\epsilon_{A A}$, the energy of an $A-A$ bond. A similar argument applies to interacts between $B-B$ segments, and 
between $A$ - $B$ segments. We can then form a surface in the blend, such that several bonds will have to be cut. If forming the surface requires that we cut $z^{\prime}$ such bonds on a lattice of spacing $b$, then the energy of forming that surface turns out to be

$$
f_{s}^{(b)}(\phi)=\frac{z^{\prime} b}{2}\left[\phi^{2} \epsilon_{A A}+(1-\phi)^{2} \epsilon_{B B}-2 \phi(1-\phi) \epsilon_{A B}\right] .
$$

So we should expect the surface energy to be at least quadratic in $\phi$. Reference [8] discusses how the phenomenological parameters expressing the surface energy ( $\mu_{1}$ and $g$ in section 2.3.3) may be related to other variables, which might be experimentally measured/estimated, by such a bond counting argument.

\subsection{Wetting in semi-infinite geometries}

In this section, the term semi-infinite geometry is meant to mean a system of infinite extent but with a bounding wall or surface, like that shown in figure 2.1(a), such that the system spans from $z=0$ to $z=\infty$, where $z$ measures the distance from a bounding wall at $z=0$.

\subsubsection{Wetting in a Three Phase System}

Cahn did the earliest systematic study of wetting, which I discussed briefly in section 2.2 [27]. Using a graphical method to analyse solutions minimising the free energy functional (2.1), Cahn determined some very general results about wetting. The three phase system of a binary fluid (phases $\alpha$ and $\beta$ ) in contact with wall (phase $x$ ) is shown in figure 2.2. Wetting ${ }^{8}$ of the wall $x$ happens when one fluid phase, say $\alpha$, is excluded from contact with the wall, such that the wall is in contact only with the other phase $\beta$, as in figures 2.7 and 2.8 (this means that $\theta \rightarrow 0$ in figure 2.2). Wetting by $\beta$, in which only $\beta$ is in contact with the wall $x$, will happen if the energy of this configuration is lower than the energy of three phase contact, in which both fluid phases $\alpha$ and $\beta$ are in contact with the wall $x$. The limit of wetting by $\beta$ should occur when the free energy of the system with three phase contact is the same as when only one phase $\beta$ is in contact with the wall $x$. Since the free energy depends on the temperature $T$, Cahn predicted that a wetting temperature $T_{W}$, less than but in the vicinity of the critical temperature $T_{C}$, is the cut-off for wetting behaviour (at $T_{W}$, the energy of the two aforementioned configurations is the same).

Figure 2.6 shows a representative phase diagram of composition $c$ against $\chi$, which for the purposes here can be considered to be the inverse temperature (however, see equation (1.29)). For $T_{W} \leq T \leq T_{C}\left(\chi_{W} \geq \chi \geq \chi_{C}\right)$, the coexisting phases with composition $c_{a}$ and $c_{b}$ (lying on the coexistence curve between

\footnotetext{
${ }^{8}$ Wetting: when one phase of a binary phase system entirely coats a surface, excluding the other phase from contact. Although wetting is strictly defined to mean that the latter configuration is stable and the phase coating the surface is infinitely thick in a semi- $\infty$ system (see main text throughout), the term is usually used more loosely to describe most scenarios when a phase coats a surface.
} 


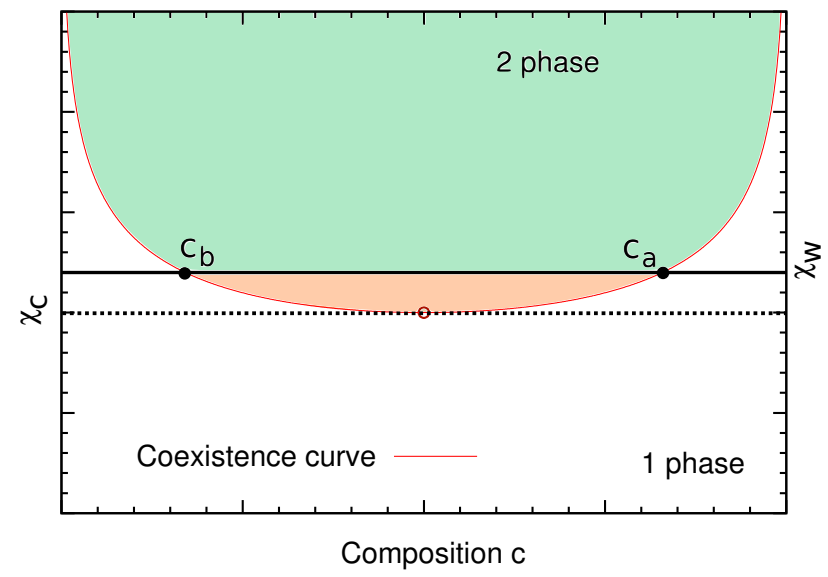

Figure 2.6: A representative phase diagram of composition $c$ against inverse temperature $\chi$. The wetting temperature $\chi_{W}$ is in the vicinity of the critical temperature. The compositions $c_{b}$ and $c_{a}$ of the coexisting phases at the wetting temperature are shown. In the two-phase region between $\chi_{W}$ and $\chi_{C}$, one of the coexisting phases will wet the surface (which one depends on the surface energy). Above $\chi_{W}$, wetting no longer occurs, only positive adsorption of the preferred phase at the surface.

the points shown and the critical point at the bottom of the coexistence curve) constitute perfect wetting, with an infinitely thick layer of the preferred phase coating the wall $x$. For $T<T_{W}\left(\chi>\chi_{W}\right)$ there is only positive adsorption of the preferred phase (an excess of the preferred phase at the wall $x$ ). At $T=T_{W}$ $\left(\chi=\chi_{W}\right)$ a first-order transition occurs, which constitutes a jump from positive adsorption to an infinitely thick layer of the preferred phase. This jump in the wetting layer thickness, from finite to infinite, is characteristic of first-order wetting. A schematic of this transition is given in figure 2.7: wetting occurs because there is a spontaneous change in which configuration has a lower energy, prompting the system to switch from a profile $c^{\text {finite }}(z)$ to $c^{\infty}(z)$. Cahn also predicted prewetting (although the term was not coined here): when one of the phases, say $\beta$, is not stable in the bulk system (because the system is still in the one phase region) this phase can still form a non-homogeneous layer of finite thickness at a surface (wetting happens in the two phase reion). When the composition of $\beta$ reaches the value for coexistence, a first-order transition can occur and the layer thickness jumps to $\infty$.

Cahn therefore showed that wetting phenomena should be observed in any twophase system in contact with a surface (i.e. three phase system) close to the critical point of unmixing.

\subsubsection{Ising Systems with a wall}

In pioneering work utilising phase portraits (essentially plots of composition and composition gradient, along with boundary conditions; see chapter 3) to study phase equilibria (composition profiles that minimise the free energy), Pandit and Wortis studied a semi- $\infty$ nearest neighbour Ising model with a bulk field and 


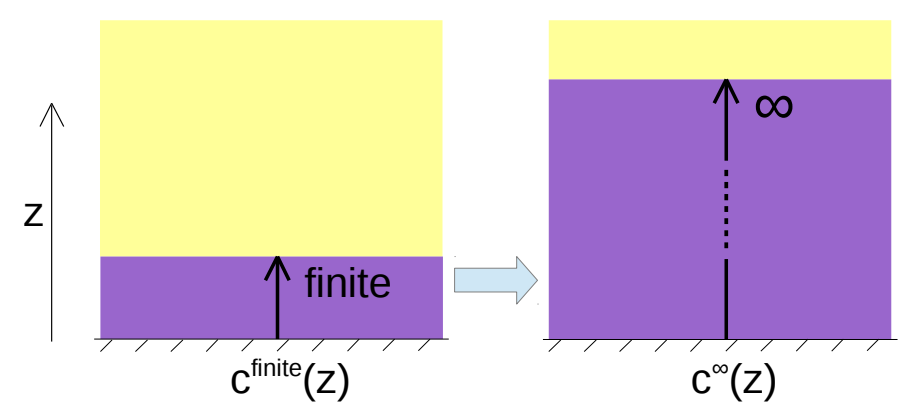

Figure 2.7: Schematic of a first-order wetting transition, which constitutes a jump in the layer thickness from a finite value to infinity. The jump occurs due to a spontaneous change in which composition profile has the lower free energy, from $c^{\text {finite }}(z)$ at $T<T_{W}$ to $c^{\infty}(z)$ at $T_{C}>T>T_{W}$, as parameters like the temperature are changed. At $T=T_{W}$, the free energies of $c^{\text {finite }}(z)$ and $c^{\infty}(z)$ are equal.

surface field (and also modified spin-spin interactions in the layer of spins adjacent to the wall) [29]. This was discussed briefly in section 2.3.1 with regards to the form of the surface energy, and the schematic of figure 2.3 still applies.

First-order wetting was found for a non-zero, large enough surface field (preferring up-spins, say) and opposing bulk field (preferring down-spins, say), in the limit that the bulk field goes to zero. At a temperature $T_{W}$ it is found that the minimal energy profile for the systems changes discontinuously from a profile with a finite wetting layer of the preferred phase to a profile in which this layer is infinite. For non-zero bulk field (which is in analogy with a 2-phase fluid mixture away from coexistence) pre-wetting transitions are found in which there is still a jump in the thickness of the wetting layer, due to a sudden change of profile of minimal energy, but this jump is from a finite value to a large but also finite value. These transitions are first-order; the characteristic jump in wetting layer thickness can be seen as due to an instantaneous switch of profiles to achieve a lower energy state, as represented in figure 2.7.

Second order wetting, which they found under the same bulk field conditions but with smaller surface fields, was also studied. This so-called 'critical-wetting' temperature (as opposed to 'wetting temperature', which they took to mean the temperature for a first order transition) is very close to the critical temperature of the system. Upon approaching the critical-wetting temperature, the wetting layer thickness goes to infinity, but continuously. This is because there is no sudden jump to a different profile of minimal energy. Instead, upon approaching this temperature, the profile continuously changes until the part of the profile corresponding to the wetting layer is infinitely thick, as shown in figure 2.8.

\subsubsection{Wetting in polymer systems}

Nakanishi and Pincus studied wetting for simple fluids in a semi-infinite geometry in which the bounding wall preferred the liquid (as opposed to the vapour) phase [31]. The work was carried over to polymer systems too. Similar to earlier work [29], a phase portrait method was used to study wetting and prewetting. 


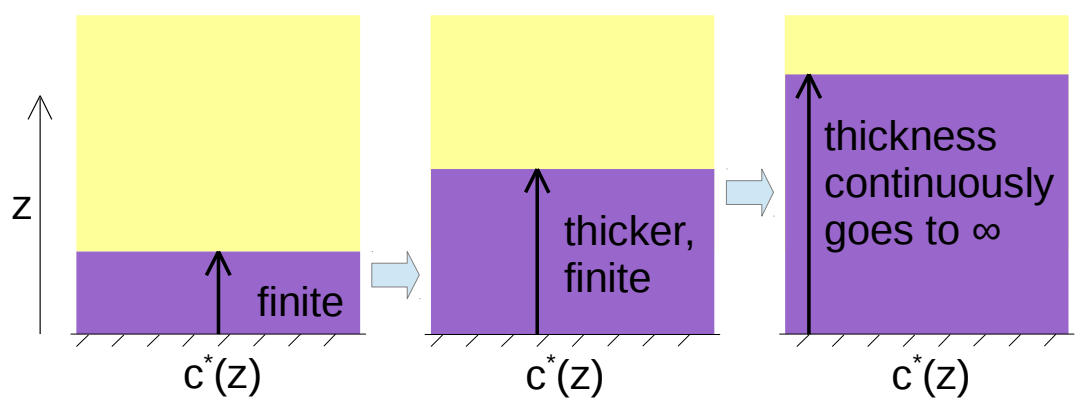

Figure 2.8: Schematic of a second-order wetting transition. As the temperature approaches the so-called 'critical-wetting' temperature, very close to the critical temperature, the composition profile $c^{*}(z)$ changes continuously such that the thickness of the wetting layer goes to infinity.

'Extended wetting' was proposed, whereby a metastably wetted state (i.e. profile) could exist below the wetting temperature. The argument for this behaviour comes from studying inflexion points in the free energy, in the same way that the spinodal is defined in infinite bulk systems. This yields surface spinodals which give rise to metastably wetted and metastably non-wetted states. However, it is noted that this behaviour could simply be artefacts from the mean-field theory. They argued that, for polymer blends, critical/second-order wetting should be impossible to observe, as should extended wetting.

Schmidt and Binder studied a semi-infinite binary polymer mixture in contact with a wall preferring one polymer species [33], using a Flory-Huggins-de Gennes free energy functional with an additional term for the bare surface energy, given by equation (2.5). First-order wetting, prewetting and second-order wetting were studied. They found that, at two-phase coexistence, the wall is always wetted with the preferred phase, but that there is strong enhancement of the preferred component at the wall even away from two phase coexistence. In disagreement with previous work by Nakanishi and Pincus [31], Schmidt and Binder argued that it should be possible to observe second-order wetting in a binary polymer mixture (in which mean-field descriptions should work well, and the spinodal should be well defined rather than smeared out by fluctuations, as it would be in simple fluid systems). The disagreement arises from a difference in the magnitude of the polymer wall interactions assumed by the authors, with the assumptions of Schmidt and Binder probably being much more physical, since the latter work assumes that the interactions between polymer segments and surfaces is of comparable magnitude to that between simple molecules and surfaces, as explained in section 2.3.3.

\subsection{Phase Separation in finite geometries}

In this section, I discuss phenomena resulting from the confinement of a nonuniform system between two walls that are a finite distance apart i.e. a film, figure 2.1(b) but with finite $d$. 


\subsubsection{Laterally Coexisting Film Profiles}

In the previous section, it was mentioned that in the non-wet state, there is positive adsorption of the prefered component at the surface, but the discussion was reserved to considering only how the composition varies with distance from the surface. Of course, if the composition was only allowed to vary in the direction perpendicular to the wall, then one would find in the non-wet state preferential adsorption at the wall, and could not find cases in which there was lateral variation. However, in real films the composition may of course vary in the direction parallel to the surface as well, and when the surface is non-wet it is actually preferable for the film to exist in a state of lateral segregation, as in figure 2.2, for which both phases $\alpha$ and $\beta$ are in contact with the surface $x$. This lateral segregation will be discussed in this section on finite systems i.e. films (although the study of lateral segregation is still possible for semi-infinite systems), and in chapter 3 .

\section{Surface Regimes}

In a film with symmetrically attracting surfaces, figure 2.9 , both surfaces prefer the same component of the blend between the surfaces. More specifically, each surface attracts a preferred component in exactly the same way as the other surface, so that if the surfaces were swapped there would be no observable difference.

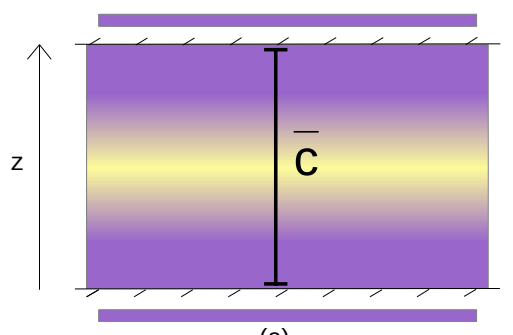

(a)

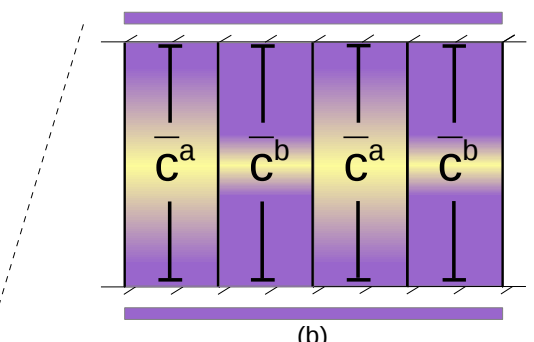

(b)

Figure 2.9: Symmetrically attracting surfaces: both surfaces prefer the same component of the blend. (a) Trilayer profile; (b) Laterally coexisting profiles.

In a film with anti-symmetrically attracting surfaces, figure 2.10 , one surface prefers one component and the other surface prefers the other component. More specifically, the surfaces attract their preferred components in exactly the same way, such that if the labels on the components of the mixture were swapped and the surfaces were swapped, there would be no observable difference.

In a film with asymmetrically attracting surfaces, the surfaces attract the components in any combination of ways that is not specifically symmetric or antisymmetric, hence asymmetrically attracting surfaces are the general case. One important idealised case is a film of finite width in which one surface preferentially attracts a component in the blend and the other surface has no preference (neutral). The profile in that case would still be similar to that in figure 2.10 if either the upper or lower surface were replaced by a neutral surface. 


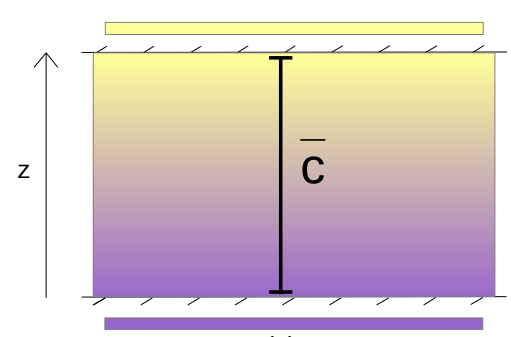

(a)

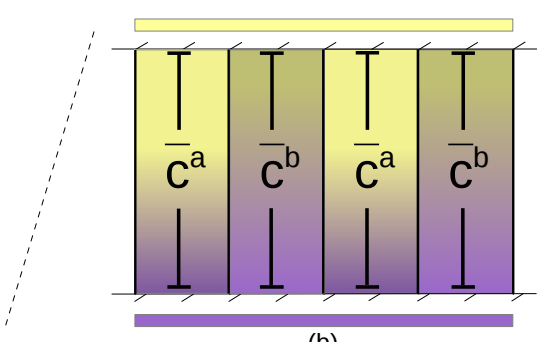

(b)

Figure 2.10: Antisymmetrically attracting surfaces: one surface prefers one component in the exactly the same way as the other surface prefers the other component. (a) Bilayer profile; (b) Laterally coexisting profiles.

\section{Neglect of contact angle}

The schematics of figures 2.9 and 2.10 show: (a) a vertically segregated film; and (b) a laterally segregated film. It is important to note that in the schematics (b) that both phases make the same angle $\theta=90^{\circ}$ with the surface, in contrast to figure 2.2 in which the non-wet state has a general angle $\theta \neq 0$. In reality, the phases in the laterally segregated state will have $\theta \neq 90^{\circ}$, but in the context of the work in this section, consideration of how these phases exist side-by-side (thus making a specific angle $\theta$ with the surface) is not included, due to the difficulty of this problem (which I address and partially solve in chapter 4). In the work discussed, the composition profiles are calculated only in the direction perpendicular to the film. Thus it is simplest to visualize laterally coexisting composition profiles as they are given in 2.9-2.10(b), which implicitly treats the coexisting phases as if they could not 'feel' lateral contact with each other. As a final word on this for now, the difficultly can be appreciated if one considers figure 2.2 in the case that the phases $\alpha$ and $\beta$ are themselves highly non-uniform, with an interface between them (hence lateral variation) and a vertical profile due to preferential attraction by the surface.

\section{Average Composition}

Also in the figures 2.9 and 2.10, the average compositions of the film profiles $\bar{c}$, $\bar{c}^{a}$ and $\overline{c^{b}}$ are represented. It is useful to consider the average composition. In the same way that the composition of a two-phase bulk (or semi- $\infty$ ) mixture can be described by the compositions $c_{a}$ and $c_{b}$ of the coexisting phases (determined by the coexistence curve, as in figure 2.6), it is useful to consider the average composition of laterally coexisting phases when describing how the presence of preferentially attracting surfaces and the finite thickness of a film affects the behaviour of the mixture in the film e.g. how does the surface preference affect the compositions of the coexisting phases? This section discusses these ideas. 


\subsubsection{Symmetric confinement}

\section{No true wetting transition}

Nakanishi studied the effects of finite film thickness for films of a binary fluid mixture confined by symmetric walls, using mean field theory for a lattice gas [31]. It was found that all the sharp transitions found in semi- $\infty$ systems are smeared out in finite systems, and that true wetting no longer occurs (note that it is still usual to use the term 'wetting' in finite systems to denote when having only one phase in contact with a surface is favourable). Considering that at the wetting transition there is a jump in the thickness of the adsorped layer from finite to infinite thickness, and that in a finite geometry no infinitely thick wetting layer can form, this is not too surprising. The only remaining wetting-type transition is a first-order, distorted version of a pre-wetting transition for semi-infinite systems, whereby there is a jump from a finite thickness to a larger, but still finite thickness of the adsorped layer. It was found that the compatibility of the fluid components increases upon decreasing the film thickness. This appears as a shift in the critical temperature of unmixing (and therefore the whole coexistence curve) to lower temperatures, meaning that the one-phase region becomes larger (more compatibility of the components). The coexistence curve is shifted to towards the component preferred by the walls, such that the coexisting phases are richer in the component preferred by the wall (since it is now possible to support compositions richer in that component).

\section{Capillary Condensation}

Binder and Landau studied capillary condensation via a lattice gas model, in which a binary liquid-vapour system is confined between symmetrically attracting surfaces (which represent a slit/pore/capillary) in order to study finite size effects i.e. effects resulting specifically from the finite width of the slit [34]. Capillary condensation is the phenomena in which a vapour condenses on the walls of a finite sized pore or slit at a chemical potential different to that at which it would condense in a bulk system, or in other words the vapour layer appears under conditions which, in the bulk, would not give rise to this liquid layer (note that 'the bulk' is meant in the sense that the slit is infinitely thick). This can happen if the surfaces prefer the liquid component since a liquid phase may exist under conditions which would have otherwise corresponded to the one-phase region. The shift of the chemical potential was found to be in agreement with the Kelvin equation $\mu_{c}(D)-\mu_{c}(\infty) \propto D^{-1}$ when $D$, the film thickness, is large enough. Monte Carlo simulations showed a transition from a non-wet state to a 'wet' state. In the wet-state there is high adsorption of the liquid at the walls for both the gas phase and the liquid phase, with the adsorption of liquid at the walls even in the gas phase being very high and approaching the adsorption of the liquid phase. The local density at the walls varies smoothly in this finite system, since the true wetting transitions of semi-infinite systems are smeared out (again, note that it is normal to refer to these phenomena as wetting, even though they are technically not wetting as defined in semi- $\infty$ systems). The 
critical point was shifted to lower temperatures as the films became thinner, such that condensation occurs at lower temperatures for thinner films (the thinner the film, the more incompatible the components of liquid or vapour have to become for the liquid to be supported, even with the preferentially attracting surfaces, since for thinner films there will be a higher cost in the gradient energy arising from the interface between vapour and liquid). Also, the coexistence curve is shifted towards higher densities i.e. towards the liquid component, since denser phases are now supported due to the walls preferring the liquid phase.

Finite thickness effects in films between symmetric walls remove transitions seen in equivalent semi-infinite systems. Instead, there is just a smooth increase in the surface excess where there would be a wetting layer formed in the semiinfinite system. For thick enough films, variations of a pre-wetting transition still exist, with a jump in the amount of excess surface material [35]. Binder suggests a definition for capillary condensation as follows: "Shift of the vapour liquid transition in a slit pore due to surface effects, so that condensation of a vapour that would be under-saturated in the bulk occurs" [36]. It can be seen from the Kelvin equation that as the thickness of the film tends to infinity the critical point for symmetric films tends to the critical temperature of the bulk system, $\left.\left(\phi_{C}(D), T_{C}(D)\right) \rightarrow\left(\phi_{C, \infty}, T_{C, \infty}\right)\right)[36]$

\section{Polymers}

Flebbe, Dunweg and Binder studied a binary polymer mixture between symmetric walls for a finite film thickness using Flory-Huggins-de Gennes mean field theory [37]. It was shown that polymer blends exhibit much of the same behaviour as blends of simple molecules, such as enhanced compatibility of the components upon decreasing film depth, a shift of the coexistence curve to lower temperatures (indicating enhanced compatibility) and compositions richer in the component preferred by the walls as the preference for that component at the walls increases. It was shown that the film thickness alone had little effect on $\bar{\phi}_{c r i t}$, the average composition of the critical phase, whereas the wall interactions had a much more pronounced effect on $\bar{\phi}_{\text {crit }}$ (since the more the walls prefer a particular component, the richer phases can be in that component, since richer phases are supported by excess at the walls). Importantly, it was shown that only symmetric composition profiles are stable, figure 2.9(a), and that coexistence of laterally separated phases should occur under certain conditions, these phases richer and poorer, respectively, in one of the polymer species, as in figure 2.9(b). Increasing the average composition of the blend towards the component not preferred by the walls will eventually stabilise the phase richer in the non-preferred phase (this sudden change of minimal energy solution being a first-order phase transition), though a significant amount of the non-preferred species in the blend is required. 


\subsubsection{Antisymmetric confinement}

\section{Freely Fluctuating Interface}

Parry and Evans took a pioneering step by studying a simple fluid or Ising magnet in a finite thickness film confined between antisymmetric walls [38]. The phase behaviour was found to be strikingly different from that for a film with symmetric walls. For finite films, coexistence of two-phases can only occur for temperatures $T<T_{C, L}$ ( $L$ is the film thickness), where $T_{C, L}<T_{W}<T_{C, \infty}, T_{W}$ being the wetting temperature of the semi-infinite system (so we could say $T_{C, L} \equiv T_{W}^{\text {finite}}$ ). Also, the thickness of the film determines whether or not the film can exist in two laterally separated phases or not; only for $L>L_{C}$ can the film exist in two phases. Parry and Evans are accredited with the discovery that for $T>T_{W}$ and large enough $L$ there is a freely fluctuating interface in the film centre, parallel to the walls. This interface is between phases rich in opposite components of the binary mixture, each phase coating the wall (forming a layer on the wall) which prefers that phase's majority component. This can be visualised with figure 2.10(a): between the different phases coating each surface, there is an interface, and the less compatible the components are, the sharper this interface will become, until at a temperature $T_{C, L}$ it is preferable for the film to exist as laterally coexisting phases, as in figure 2.10(b).

\section{Interface Unbinding}

Albano and Binder investigated the freely fluctuating interface, predicted by Parry and Evans, using a Monte Carlo simulation of an Ising model between antisymmetric walls [39]. One wall interacted with up-spins exactly as the other wall interacted with down-spins. The results showed that in the non-wet state, $T<T_{W}(D)<T_{W}(\infty)$ (where $D$ is the film depth), the interface between the up-spins and down-spins is 'bound' to either wall (with no preference due to the perfect antisymmetry), whereas in the wet state, $T>T_{W}(D)$, the interface is 'unbound', and there is "an interface position fluctuating around the centre of the film... (which) slowly diffuses back and forth across the strip" during the simulation (the strip needed to be wide enough to accommodate this interface). Wetting can thus be viewed as 'interface unbinding'. A definition from Binder is that a wetting transition is a "singularity of the surface excess free energy of a semi-infinite system at condition of two-phase coexistence, characterised by the unbinding of a flat interface from a confining wall" [36]. Of course, given the finite system size, the wetting layer cannot be infinitely thick, so this transition is a finite-sized equivalent of wetting.

\section{Soft-mode}

Parry and Evans built upon their previous work to investigate a 'soft-mode' existing for $T_{W}<T<T_{c b}$, ( $T_{c b}$ is the critical temperature for a bulk system), using a phase portrait approach to investigate the phase equilibria of the system [40]. The soft-mode is characterised by an interface between layers rich in one component of the blend, each phase rich one component of the blend, such that each 
phase coats the surface that prefers that component (so the soft-mode has the freely fluctuating interface discussed above). Since variation of the magnitude of the surface fields can push the wetting temperature arbitrarily below the critical bulk temperature, this soft-mode phase can exist for a wide range of temperatures. As the temperature is brought close to the critical bulk temperature from below, $T \rightarrow T_{c b}^{-}$, the width of this interface tends to $D$, the width of the film, so that the interface ceases to become well defined, and the entire film profile is essentially interface (a single layer with a composition gradient, rather than two clearly distinct layers with an interface between them). For antisymmetric walls, it was shown that as $D \rightarrow \infty$, the critical temperature required for separation into coexisting phases tends to the wetting temperature, not the critical bulk temperature. This is distinct from films between symmetric walls.

\section{Polymers}

Souche and Clarke developed a phase portrait approach to study binary fluid systems in a finite film geometry, with focus on a binary polymer blend confined between antisymmetric walls [41]. A Hamiltonian formulation was used to generate the phase portraits, which allowed for an easy graphical study of the phase equilibria (this method is the subject of chapter 3). The phase portraits allow easy visualisation of the soft-mode transition and the laterally coexisting phases. Souche and Clarke extended their work to ternary systems, namely a binary polymer mixture with solvent, in a finite antisymmetric wall geometry [42]. This work showed that the stable solutions are all analogues of the solvent-free system. Increased amounts of solvent act to increase compatibility of the polymers, and hence play a similar role to temperature. The soft-mode transition from an approximate monolayer film to a bilayer structure, figure 2.10(a), as well as lateral phase separation into laterally coexisting phases, figure 2.10 (b), can be induced by lowering the temperature and/or the solvent concentration.

\subsubsection{Asymmetric confinement}

Binder, Landau and Ferrenberg studied Ising strips confined between asymmetric walls using Monte Carlo simulations [43]. The results were qualitatively the same as those for strips between antisymmetric films, provided that the walls serve to attract oppositely aligned spins strongly enough, so that the profiles are still similar to those of figure 2.10, i.e., approximately antisymmetric. Due to the broken (anti)symmetry, coexistence of the phases in which the interface is bound to one wall or the other now occurs for non-zero bulk field. For $T>T_{W}(D)$ there is no ordering whatsoever (i.e. the average magnetisation is zero) despite the lack of symmetry which would otherwise suggest there might be some ordering towards the most preferred (least not-preferred) spins, so only at $T=T_{W}(D)<T_{W}(\infty)$ is there symmetry breaking and a finite magnetisation [44]. The results are exactly in analogy with the 'interface-localisation transition' of Parry and Evans [38, 40]. 


\section{Crossover Behaviour}

Muller, Binder and Albano studied the intermediate cases between perfect antisymmetry and perfect symmetry, i.e. general asymmetry, with the aim of demonstrating the crossover behaviour between capillary-condensation behaviour (symmetric films) and interface-localisation transition behaviour (antisymmetric films) [45]. Self-consistent field calculations were used, which showed that the crossover is gradual but non-monotonous. Keeping the interaction at one wall fixed, it was shown that the critical temperature of the film increases from a minimum for antisymmetric walls, to a maximum approximately when one surface is neutral. The critical temperature then decreases again, but remaining far above the critical temperature for antisymmetric walls, as the wall interactions are tuned to perfect symmetry. As the walls are brought from perfect antisymmetry to perfect symmetry, such that the wall that attracts species B (in the antisymmetric case) is changed slowly to a neutral surface and then to an A attracting surface (bringing about the symmetric case), there comes a point at which only capillary condensation is possible, in which only prewetting-like enrichment of the wall(s) by $\mathrm{A}$ is possible, at a modified temperature and chemical potential from that for a bulk system.

\subsection{Summary}

In this chapter I describe the theory of multicomponent systems in contact with a surface which may preferentially attract a particular component. I discussed literature for Ising systems, simple fluid systems, and polymer systems, showing that the description of the surface-blend interaction energy is similar in all cases. I discussed the concept of wetting, several different surface energy regimes, and the concept of a vertically segregated film and a laterally segregated film. The next chapter, which begins the discussion of my own research in this thesis, uses this theory to introduce the problem of solving for equilibrium profiles of polymerblend thin films with preferentially attracting surfaces. 


\section{Hamiltonian Phase Portraits FOR Polymer-Blend Thin Films}

I describe a Hamiltonian Phase Portrait method for studying the equilibrium profiles of polymer-blend thin films, including an extension I made to this method which made it suitable for general (asymmetric) wall-blend interaction regimes, rather than only regimes with convenient symmetries. I derive the equations that equilibrium film profiles must satisfy and the expressions for the phase portraits. I describe how the phase portraits can be used to visualise how the equilibrium profiles change with temperature regime and film depth, and discuss a thermodynamic mechanism of surface roughening based on laterally coexisting profiles having different depths to minimise the free energy. This work was published in my first paper "Surface roughening in polymer blend thin films by lateral phase separation: A thermodynamic mechanism" [46].

3.1 Introduction . . . . . . . . . . . . . . . . . . . 46

3.2 Phase Equilibria . . . . . . . . . . . . . . . . . . . . . . . . 49

3.3 Hamiltonian Phase Portraits . . . . . . . . . . . . . . . . . 52

3.4 Phase Equilibria of Asymmetric Films . . . . . . . . . . . . . . . 59

3.5 Surface Roughening . . . . . . . . . . . . . . . . . . 67

3.6 Summary . . . . . . . . . . . . . . . . . . 70 


\subsection{Introduction}

This chapter describes a graphical method for studying polymer-blend films: Hamiltonian Phase Portraits. This method allows equilibrium film profiles, describing the composition of a polymer-blend between selectively attracting surfaces, to be visualised and calculated in phase space. This provides insight on how equilibrium profiles change as film depth and temperature change (and is also suitable to study how profiles change as surface-blend interactions change, although this is not discussed here, but is a part of chapter 4). The work in this chapter is a direct extension of the Hamiltonian Phase Portrait method of Souche and Clarke [41], who were the first to use the method to study polymer-blend thin films of finite thickness with this method, for films with very particular symmetries which simplified the problem. My work allowed the Hamiltonian Phase Portrait method to be extended to general cases, and was published in a paper discussing both this extension and a possible mechanism of surface roughening in polymer-blend thin films [46].

\subsubsection{Equilibria of Polymer-Blend Thin Films}

This chapter concerns binary polymer-blends of components $A$ and $B$ between selectively attracting surfaces/walls. The composition of the film at any point can be described by $\phi \equiv \phi_{A}$, and 'selectively attracting walls' means that the walls prefer to be in contact with fluid at a particular composition (section 2.5 contains a discussion of selectively attracting walls and different 'surface regimes'). The profile $^{9} \phi(z)$ of a polymer-blend thin film describes the volume fraction $\phi$ (composition) as a function of position $z$ in the film, which is usually measured from one of the film surfaces.

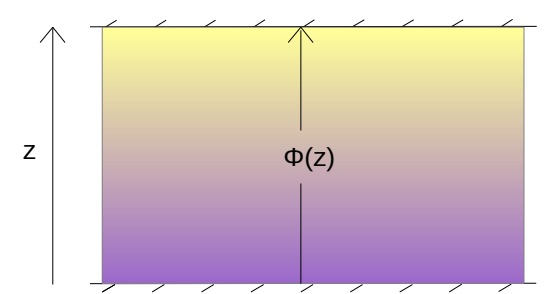

(a)

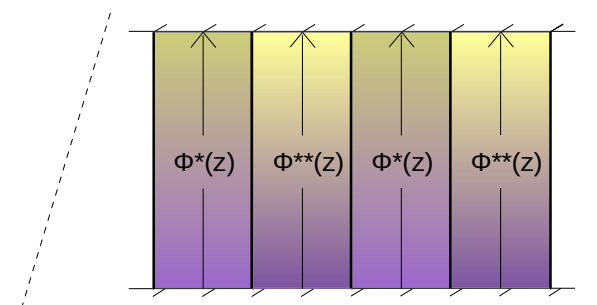

(b)

Figure 3.1: Schematic of a binary polymer-blend film. The hatched lines represent a confining surface/wall, and the composition of the blend varies with $z$, which measures the position in the film (usually the distance from one of the walls). (a): independently-existing equilibria, in which the profile $\phi(z)$ describes the entire film; (b): coexisting equilibria $\phi^{*}(z)$ and $\phi^{* *}(z)$ which exist side-by-side (laterally coexisting). Note that in this schematic, the interface between the coexisting phases is not displayed. In reality, there will be an interface between these phases, this interface making an angle with the surfaces as in figure 2.2.

\footnotetext{
${ }^{9}$ Profile: description of a polymer-blend by volume fraction $\phi$ as a function of spatial variables e.g. $\phi(z, y)$, where $z$ is the vertical (depth) dimension and $y$ is the lateral (parallel to substrate) dimension. Throughout this thesis the profiles are discussed primarily in 1D as $\phi(z)$.
} 
It is useful to calculate the equilibrium profiles, which are profiles for which the free energy is a minimum. I shall often refer to equilibrium profiles as phase equilibria ${ }^{10}$. Since a system will naturally evolve to minimise its free energy, knowledge of equilibria informs us about how the system will evolve: which state ${ }^{11}$ the system will end up in, whether a particular state is stable or not, and even insights into the kinetics by which the system will evolve towards and between equilibrium states. The subject of this chapter is the visualisation and calculation of equilibria using Hamiltonian Phase Portraits.

Figure 3.1 is a schematic of a polymer blend between selectively attracting surfaces, in which several profiles are represented in such a way that we assume that the composition varies only in the vertical 'depth' dimension, even in the case that several profiles coexist side-by-side in the film (the neglect of how the composition varies laterally, such that the interface between coexisting phases is not taken into account in the calculation of $\phi(z)$, is discussed in section 2.5). Note that films may be described by independently-existing equilibria ${ }^{12}$ as in Figure 3.1(a), or by (laterally) coexisting equilibria ${ }^{13}$ as in Figure 3.1(b).

\subsubsection{Phase Space, Trajectories, and Phase Portraits}

The Phase Space $^{14}$ of a system is a space of the variables that describe that system. For example, a particle can be described by its position and its momentum, and so a plot of position and momentum would constitute a phase space for the particle. The unique state of the particle at any time will be a point in that phase space. If the particle is moving, it will trace out a Trajectory in phase space, consisting of all the points of position and momentum that it had as it moved. If we knew the trajectory (path) through phase space, we could calculate the time required for the particle to move along any part of that trajectory. A polymer blend film has an analogy with a moving particle: position $\rightarrow$ composition, momentum $\rightarrow$ composition gradient, time $\rightarrow$ distance. The trajectory of a polymer film would consist of a curve of composition and composition gradient over a length (depth) of film. A Phase Portrait for a polymer film is a plot of trajectories that minimise the free energy of the film, such that equilibria are contained in the phase portraits.

\footnotetext{
${ }^{10}$ (Phase) Equilibria: profiles which minimise the free energy of the film, and therefore correspond to equilibrium. The term phase is used in analogy with bulk blends e.g. an $A-B$ blend can exist as: a miscible blend (one-phase); or $A$-rich and $B$-rich phases (two-phase).

${ }^{11}$ State(s): (disambiguation) the overall configuration of the film, and/or the equilibria contained in that configuration e.g. a film in a laterally segregated state will consist of laterally coexisting states. Non-specific term.

${ }^{12}$ Independently-Existing Equilibria: profiles which describe the film entirely and do not coexist with other profiles, as for a laterally homogeneous film.

${ }^{13}$ Coexisting Equilibria: profiles which coexist together, describing different phases in the film, as for a laterally inhomogeneous film of two laterally segregated phases.

${ }^{14}$ Phase Space, Trajectory, Phase Portrait: definitions in main text and terminology section.
} 


\subsubsection{Previous Work}

Much of the previous work that utilised a phase portrait method to study nonuniform systems in contact with a surface(s) was discussed in sections 2.3-2.4, although the discussion concerned the findings rather than the method. For completeness and context, I will return very briefly to several studies here.

Pandit and Wortis undertook pioneering work in which they developed a graphical method useful for analysing the possible solutions of mean-field theories, for the particular case a of magnetic system with an inhomogeneity in one direction [29]. They studied an Ising system with a free surface that could modify the magnetic field and exchange coupling between neighbouring spins. They showed that the equilibrium states of the system, along with first order and second order phase transitions could be easily visualised using phase portraits. Nakanishi and Pincus utilised the method of Pandit and Wortis to study wetting transitions for simple fluid systems in contact with a wall/surface [31]. Their analysis included a discussion of possible metastably wet states, and the idea of extended wetting and 'surface spinodals'. They also discussed polymer containing systems. Once again, transitions from one equilibrium state to another could be easily visualised using phase portraits. Parry and Evans studied the phase behaviour of a simple fluid or Ising magnet confined between two confining walls that exerted opposing surface fields, finding that wetting and coexistence phenomena were very different than for the same systems confined between two walls that exerted the same surface fields [40]. Although they didn't use phase portraits, they did utilise a graphical method in a similar vein as the aforementioned work above.

Souche and Clarke developed the Hamiltonian Phase Portrait method to study binary polymer-blend films, with a focus on a symmetric blend confined between antisymmetric walls [41]. This was the first time that such a technique had been applied to films of finite thickness. The phase portraits provided excellent visualisation of the soft-mode transition and laterally coexisting phase equilibria for this surface regime (antisymmetric). Souche and Clarke also applied the method to ternary systems, namely a binary polymer mixture with solvent in an antisymmetric wall regime, showing that the stable solutions are all analogues of the solvent-free (binary polymer-blend) system [42]. However, they did not extend the work to asymmetric films, for which the wall-blend interactions were not antisymmetric and/or for which the blend was not symmetric.

\subsubsection{Asymmetric Films: a more difficult problem}

Considerable attention has been paid to symmetric binary blends of small molecules or polymers, $A$ and $B$ say, confined between antisymmetric walls (for a blend $A: B$, one wall attracts $A$ in exactly the same way as the other wall attracts $B$ ). The reason for this focus is that the symmetries of both the blend and the walls greatly simplifies the study of phase equilibria: without an explicit consideration of material conservation (requiring that the profiles satisfy $\phi \overline{(} z)=\bar{\phi}$, the composition of the blend) the equilibrium profiles naturally conserve the blend ratio $\bar{\phi}=1 / 2$ for a symmetric blend, and laterally coexisting equilibria of $A$-rich and $B$-rich phases are mirror images of each other (having equal heights and equal ex- 
cess surface free energies $[40,45])$. These conveniences vanish for a polymer-blend (whether symmetric or otherwise) confined between asymmetric walls. The easiest way to show why this is the case is to address the general problem of solving for equilibria of polymer-blend thin films between selectively attracting walls.

\subsection{Phase Equilibria}

For polymer-blend thin films between selectively attracting walls, the task is to find the profiles $\phi(z)$ that minimise the free energy i.e. the task is to find profiles $\phi(z)$ that are phase equilibria. This section describes the Euler-Lagrange treatment of a film described by the Flory-Huggins-de Gennes free energy, which results in an Euler-Lagrange equation that equilibrium profiles must satisfy, a boundary condition at each surface/wall, and a material constraint.

\subsubsection{Free energy functional}

The Flory-Huggins-de Gennes free energy is discussed in section 1.5 (bulk theory) and section 2.3.3 (with surface energy terms). Here, I use the notation used by Souche and Clarke [41], with some minor changes to maintain consistency in my thesis. In one dimension, the Flory-Huggins-de Gennes free energy of a binary polymer blend confined between walls at $z=0$ and $z=d$ is given in units of $k_{B} T$ by

$$
\begin{aligned}
\mathcal{F}[\phi(z)] & =\frac{1}{a} \int_{0}^{d}\left[f_{F H}(\phi)+\kappa(\phi)(\nabla \phi)^{2}\right] d z+f_{0}^{*}\left(\phi_{0}\right)+f_{d}^{*}\left(\phi_{d}\right) \\
& =\frac{1}{a} \int_{0}^{d}\left[f_{F H}(\phi)+\kappa(\phi)(\nabla \phi)^{2}\right] d z+\frac{1}{a} f_{0}\left(\phi_{0}\right)+\frac{1}{a} f_{d}\left(\phi_{d}\right),
\end{aligned}
$$

where $\nabla \phi \equiv \partial \phi / \partial z$, the partial derivative of $\phi$ with respect to $z$, where $z$ is the distance from the wall at $z=0$. The film composition depends only on $z$ (such that the profiles $\phi(z)$ that we calculate are laterally uniform, even if these profiles are in fact coexisting with other profiles in a laterally segregated film; see figure 3.1). $a$ is the cell-spacing of the underlying Flory-Huggins lattice, which has been factored out of $f_{S}^{*}$ so that $f_{S}$ are the surface energies per unit cell $(S=0, d)$. The Flory-Huggins free energy for a binary polymer blend is

$$
f_{F H}(\phi)=\frac{\phi}{N_{A}} \ln (\phi)+\frac{1-\phi}{N_{B}} \ln (1-\phi)+\chi \phi(1-\phi),
$$

where $\phi$ is the volume fraction of component $A, N_{A}$ and $N_{B}$ are the degrees of polymerisation of components $A$ and $B$ respectively, and $\chi$ is the Flory-Huggins interaction parameter. The gradient coefficient is

$$
\kappa(\phi)=\frac{a^{2}}{36 \phi(1-\phi)} .
$$

As discussed in chapter 2 , the surface energies are given by $(S=0, d)$

$$
f_{S}\left(\phi_{S}\right)=h_{S} \phi_{S}+\frac{1}{2} g_{S} \phi_{S}^{2}
$$


where $h_{S}$ and $g_{S}$ are phenomenological parameters, taking account of blend-wall interactions and missing neighbour effects respectively. Note that only the local volume fraction at the confining walls enters into the surface energies.

\subsubsection{Euler-Lagrange equation}

Using equation (3.1) we can develop an Euler-Lagrange equation, the solutions to which are equilibrium profiles $\phi(z)$ minimising the free energy. The Lagrangian density is the integrand of Eq. (3.1):

$$
\mathcal{L}(\phi, \nabla \phi)=a^{-1}\left[f_{F H}(\phi)+\kappa(\phi)(\nabla \phi)^{2}\right] .
$$

Without constraints on the system, an Euler-Lagrange equation can be developed from Eq. (3.5) as follows (constraints will be included later in section 3.2.4). Using the notation $\dot{\phi} \equiv \nabla \phi$, the Euler-Lagrange equation is given by

$$
\frac{\partial L}{\partial \phi}-\frac{d}{d z}\left(\frac{\partial L}{\partial \dot{\phi}}\right)=0
$$

For the first term we find

$$
\frac{\partial L}{\partial \phi}=a^{-1}\left[\partial_{\phi} f_{F H}+\partial_{\phi} \kappa(\dot{\phi})^{2}\right]
$$

$\partial_{\phi} f$ is the partial derivative of $f$ with respect to $\phi$. For the second term, we take two steps. Before taking the derivative with respect to $z$, we find

$$
\frac{\partial L}{\partial \dot{\phi}}=a^{-1}[2 \kappa \dot{\phi}]
$$

and then performing the derivative with respect to $z$, we find

$$
\frac{d}{d z}\left(\frac{\partial L}{\partial \dot{\phi}}\right)=\frac{\partial}{\partial z}\left(\frac{\partial L}{\partial \dot{\phi}}\right)+\frac{\partial}{\partial \phi}\left(\frac{\partial L}{\partial \dot{\phi}}\right) \dot{\phi}+\frac{\partial}{\partial \dot{\phi}}\left(\frac{\partial L}{\partial \dot{\phi}}\right) \ddot{\phi} .
$$

Since there are no explicit appearances of $z$, the first term on the right-hand side is zero (the second two terms accounting for the dependence on $z$ through $\phi$ and $\dot{\phi})$, and so we have

$$
\frac{d}{d z}\left(\frac{\partial L}{\partial \dot{\phi}}\right)=a^{-1}\left[2 \partial_{\phi} \kappa(\dot{\phi})^{2}+2 \kappa \ddot{\phi}\right]
$$

Returning to Eq. (3.6) (factor $a$ will cancel out) we then have the result

$$
\partial_{\phi} f_{F H}=\partial_{\phi} \kappa(\dot{\phi})^{2}+2 \kappa \ddot{\phi} .
$$

(The same result can be found be directly calculating the functional derivative, which is done rigorously in the next chapter, and which is discussed briefly in section 3.3.4). Profiles $\phi(z)$ which satisfy equation (3.11) minimise the bulk free energy (note that the surface energy terms have not entered into the Euler-Lagrange equation), but phase equilibria for films must also satisfy other requirements. 


\subsubsection{Surface Boundary Conditions}

Taking the functional derivative of Equation (3.1) with respect to $\phi(z)$ to be constant at equilibrium introduces two boundary conditions, one at each confining wall, given by

$$
\begin{aligned}
& +2 \kappa\left(\phi_{0}\right) \nabla \phi_{0}=+\frac{\partial f_{0}}{\partial \phi} \equiv+h_{0}+g_{0} \phi_{0}, \\
& +2 \kappa\left(\phi_{d}\right) \nabla \phi_{d}=-\frac{\partial f_{d}}{\partial \phi} \equiv-h_{d}-g_{d} \phi_{d} .
\end{aligned}
$$

Since these boundary conditions are found in the literature as standard (having been derived by Cahn for a wall at $z=0$, equation (2.2), as discussed in chapter 2 ), and a rigorous derivation of them is given in section 4.3.1, I refrain from deriving them here.

\subsubsection{Material Constraints}

A binary blend can be described by an average volume fraction $\bar{\phi}$ of one of its components. When calculating a film profile $\phi(z)$, it is generally necessary to ensure that the film profile calculated has the average volume fraction of the blend, since this is required for material conservation. This would be required for an independently-existing profile, like that in Figure 3.1(a). A constraint equation can be written as

$$
\frac{1}{a} \int_{0}^{d}(\phi(z)-\bar{\phi}) d z=0 .
$$

(it is convenient to include the factor $a^{-1}$ ). We must construct a new Lagrangian which takes the constraints into account:

$$
\mathcal{L}^{\prime}=\mathcal{L}+a^{-1} \lambda C
$$

$\lambda$ is a Lagrange multiplier and $C$ is an expression that accounts for the constraint (3.14). It is simplest to choose the constraint $C=\phi$, since $\bar{\phi}$ in Eq. (3.14) is a constant. Using $\mathcal{L}^{\prime}$, the Euler-Lagrange equation for the system is then simply

$$
2 \kappa \ddot{\phi}+\partial_{\phi} \kappa(\dot{\phi})^{2}=\partial_{\phi} f_{F H}+\lambda .
$$

$\lambda$ must be chosen such that Eq. (3.14) is fulfilled by the profile solution $\phi(z)$. Therefore equilibrium profiles (equilibria) $\phi(z)$ must satisfy the Euler-Lagrange equation (3.16), the boundary conditions at each wall/surface (3.12) and (3.13), and the material constraint (3.14). It is because of this plethora of requirements that a phase portrait method is useful. 


\subsection{Hamiltonian Phase Portraits}

The phase portraits developed by Souche and Clarke are Hamiltonian phase portraits, plotted not with the composition and composition gradient of the film, but with the canonical position $q$ and canonical momentum $p$. However, as will be shown below, the canonical position is equivalent to the composition, and for convenience it will suffice to think of the canonical momentum as the composition gradient. The convenience of this formalism will become apparent.

\subsubsection{Hamiltonian formulation with constraints}

We can easily shift from a Lagrangian formulation to a Hamiltonian formulation, and in order to honour the constraints the (constrained) Hamiltonian must be defined using the constrained Lagrangian $\mathcal{L}^{\prime}$ :

$$
\mathcal{H}(q, p)=p \dot{q}-\mathcal{L}^{\prime}(q, \dot{q})
$$

where $q=\phi$ and $p$, the canonical momentum, is given by

$$
p=\frac{\partial \mathcal{L}^{\prime}}{\partial \dot{q}}=a^{-1} 2 \kappa(q) \dot{q}
$$

It is slightly unusual to transform to a Hamiltonian description using a constrained Lagrangian; this was an important step that I recognised as necessary if the constraints were to appear in the phase portraits. The Hamiltonian is then given by

$$
\begin{aligned}
\mathcal{H}(q, p) & =p \dot{q}-\mathcal{L}^{\prime}(q, \dot{q}) \\
& =p \dot{q}-a^{-1}\left[f_{F H}(q)+\kappa(q) \dot{q}^{2}\right]-a^{-1} \lambda q \\
& =\left(a^{-1} 2 \kappa(q) \dot{q}\right) \dot{q}-a^{-1}\left[f_{F H}(q)+\kappa(q) \dot{q}^{2}\right]-a^{-1} \lambda q \\
& =a^{-1}\left\{\kappa(q) \dot{q}^{2}-f_{F H}(q)-\lambda q\right\} \\
& =a^{-1}\left\{9 q(1-q) p^{2}-f_{F H}(q)-\lambda q\right\},
\end{aligned}
$$

Using Eq. (3.18) simplifies Eqs. (3.12) to

$$
\begin{aligned}
& a p_{0}=+h_{0}+g_{0} q_{0}, \\
& a p_{d}=-h_{d}-g_{d} q_{d},
\end{aligned}
$$

\subsubsection{Generating Hamiltonian Phase Portraits}

Since $z$ does not explicitly appear in the Hamiltonian (3.19), the Hamiltonian density is conserved [47, 29], thus $\mathcal{H}(q, p)=\mathcal{H}$ (where $\mathcal{H}$ is a constant). Eq. (3.19) can then be rearranged to give an analytical expression for the phase portraits which lie in the space $(q, p)$ :

$$
p(q)= \pm \frac{1}{3} \sqrt{\frac{a \mathcal{H}+f_{F H}(q)+\lambda q}{q(1-q)}},
$$


Particular phase portraits can be generated by choice of $\mathcal{H}$ and $\lambda$. The phase portraits consist of the flow of canonical coordinates $(q, p)$ which minimise the bulk free energy of the functional $\mathcal{F}(3.1)$, i.e., the integral in equation (3.1) is minimised, the surface energy terms not entering into the expression for the phase portraits. I will often refer to the flow of coordinates as the Hamiltonian Flow. Trajectories along these portraits take paths which minimise the free energy, since trajectories are paths through phase space that follow the Hamiltonian flow of the phase portraits. Furthermore, the boundary conditions at the surfaces can be plotted in the Hamiltonian phase space, and appear as straight lines. Before directly discussing the phase portraits, I will give some context to how they relate to the problem of calculating phase equilibria, so that the discussion of phase portraits will hopefully seem less arbitrary.

\subsubsection{Calculation of Equilibria}

How do these phase portraits relate to the calculation of equilibrium profiles $\phi(z)$ ? If we begin at a point $q_{i}$ somewhere on a particular phase portrait (determined by $\mathcal{H}$ and $\lambda$ ), we can move along the Hamiltonian flow up to a point $q_{j}$, whilst performing the path integral

$$
z=\int_{q_{i}}^{q_{j}} \frac{d q}{\dot{q}} .
$$

Since there is no constant of integration, $q_{i}$ corresponds to $z=0$, and integrating up to $q_{j}$ will give a value of $z=L$ that corresponds to a length of film over which we have integrated. If we imagine discretising the interval $q_{i} . . q_{j}$ into steps $q_{i}, q_{i+1}, q_{i+2} . . q_{j}$, such that the integration 3.24 becomes a sum of sub-integrals, then for every value of $q$ between the limits $q_{i}$ and $q_{j}$, we will have a unique value of $z$ : we then have a set of pairs of $q$ and $z$ values, therefore we will have calculated $\phi(z) \equiv q(z)$, a profile minimising the bulk free energy. This film profile has $\phi(z=0)=q_{i}$ and $\phi(z=L)=q_{j}$.

Since the boundary conditions (3.12)-(3.13) must be satisfied by equilibria, we can only choose a value of $q_{i}=q_{0}$ on the Hamiltonian flow which coincides with the boundary condition at $z=0$. Similarly, we can only integrate up to $q_{j}=q_{d}$, a value of $q$ for which the flow coincides with the boundary condition at $z=d$. This being the case, to calculate equilibrium profiles of films we must begin at a point $q_{0}$ on the $z=0$ boundary condition and move along the Hamiltonian flow until we reach a point on the $z=d$ boundary condition, which gives us our upper limit $q_{d}$. Thus we will have performed the following path integral

$$
z=\int_{q_{0}}^{q_{d}} \frac{d q}{\dot{q}},
$$

and calculated the profile $\phi(z)$ for a film of depth $d$. Figure 3.2 is an example phase portrait (left) with a valid equilibrium trajectory marked by the bold coloured line, alongside the profile (right) that can be calculated via the integration (3.25) (phase portraits, along with a choice of parameters, will be discussed properly later. Figure 3.2 is present here only for visualisation purposes). To summarise, solution trajectories of equilibrium film profiles $\phi(z)$ are those parts of the phase 
portraits which flow between the wall boundary conditions i.e. which begin on the $z=0$ boundary condition and end on the $z=d$ boundary condition. The boundary conditions are satisfied when they cross the Hamiltonian flow.
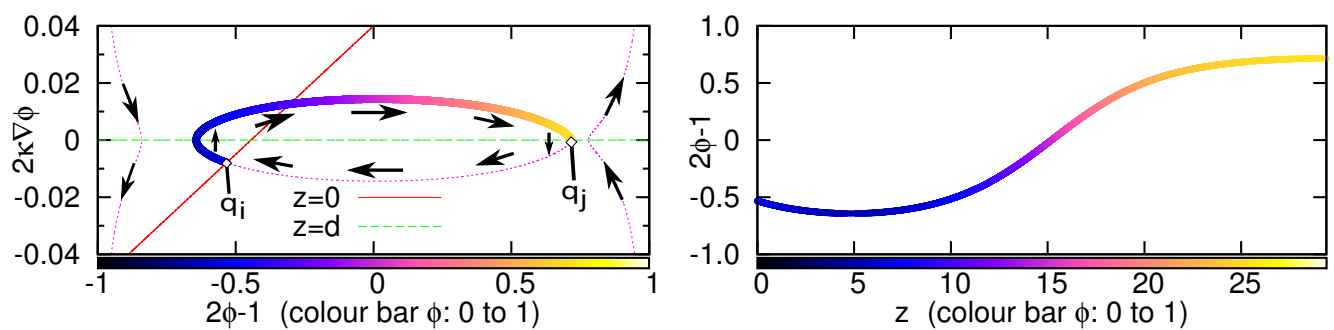

Figure 3.2: An example phase portrait (left) and corresponding equilibrium profile (right), shown for visualisation (figures from bottom of Fig. 3.7). The boundary conditions are straight lines in this phase space. The bold line shows the profile trajectory that satisfies the boundary conditions, such that $q_{i} \equiv q_{0}$ and $q_{j} \equiv q_{d}$. Arrows indicate the direction of increasing $z$ along the Hamiltonian flow. The $x$-axis is scaled to $2 \phi-1$ in keeping with reference [41].

Note that the Hamiltonian flow has a clockwise direction to it (to move in the direction of increasing $z$ ): the orbital part of the flow should be followed in a clockwise manner; the left edge should be followed from top to bottom; and the right edge from bottom to top; see figure 3.2. Not present in this particular phase portrait are parts of the flow that would be above and below the orbital part. These parts can be seen in figures 3.3.4(b)-3.3.4(d): the top part should be followed left to right and the bottom part from right to left. Also note that the path integral does not have to stop at the first point when the trajectory crosses the boundary condition for $z=d$, as long as it ends on the boundary condition for $z=d$, as in figure 3.2 (similarly, we can choose which cross with the $z=0$ boundary condition that we begin our integration from). Equilibria for a particular blend with average volume fraction $\bar{\phi}$ must also satisfy the material constraint 3.14, which I have not yet discussed, but any trajectories that follow the Hamiltonian flow between the boundary conditions are valid equilibria.

\section{Drawback and Advantage}

The drawback of the Hamiltonian Phase Portrait method is that we can't know the depth or composition of the profile we are calculating in advance of calculating it. We must choose values for $\mathcal{H}$ and $\lambda$ (some of which won't generate phase portraits that cross the boundary conditions) and calculate profiles, which will yield particular $\phi(z)$. As can be seen from figure 3.2, there are a number of different possible trajectories that could be made that begin on the $z=0$ boundary condition and end on the $z=d$ boundary condition. This is generally the case for any particular phase portrait. It is precisely because of this that the Hamiltonian Phase Portrait method is useful, which will hopefully become apparent later. 


\subsubsection{Description of Phase Portraits}

\section{Parameters: Symmetric Blend between Asymmetric Surfaces}

Throughout the remainder of this chapter, I will specialise to a symmetric binary polymer blend $\left(N_{A}=N_{B}=N=100\right)$ confined between a B-attracting wall $\left(h_{0}=-0.05 ; g_{0}=0.18\right)$ at $z=0$ and a neutral wall $\left(h_{d}=0.0 ; g_{d}=0.0\right)$ at $z=d$. This means that the $z=0$ wall prefers fluid at composition $\phi<0$, whilst the $z=d$ wall has no preference since the surface energy of this wall has no dependence on the local volume fraction. This specialisation is made in order to isolate the effects of wall asymmetry, and simplifies my discussion of the phase portraits. From now on I will set $a=1$ for convenience (effectively, $z$ is then in units of $a$ ). I will assume that $\chi=A+B / T$, and so use $\chi$ to discuss temperature. For the symmetric blend, the bulk critical temperature is $\chi_{c b}=2 / N=0.020$.

\section{Characterising Phase Portraits: Fixed Points and the Separatrix}

Figure 3.3 shows examples of Hamiltonian phase portraits plotted for $\lambda=0$, for three temperature regimes: (b) $\chi=0.015$ (above the critical bulk temperature $\chi<\chi_{c b}$ ); (c) $\chi=0.021$ (below the critical bulk temperature and above the wetting temperature $\chi_{c b}<\chi<\chi_{w}$ ); and (d) $\chi=0.026$ (below the wetting temperature $\left.\chi_{w}<\chi\right)$. For estimation of $\chi_{w}$, see the discussion of $\lambda=0$ phase portraits below. Figure 3.3(a) shows three 'separatrix' (I use the terminology of reference [41]), the shape of which characterises the phase portraits (the separatrix are also plotted in figures 3.3(b)-(d)). The relevance of these temperature regimes and their relation to the separatrix is discussed shortly. The phase portraits are symmetric around $p=0$ and $2 q-1=0$; this is due to the symmetry of the polymer blend and setting $\lambda=0$. Also plotted are the boundary conditions for a $B$-attracting wall at $z=0$ and a neutral wall at $z=d$.

Figure 3.3(a) shows only the separatrix for three values of $\chi$, each value corresponding to a different temperature regime. What are these separatrix? The separatrix ${ }^{15}$ are phase portraits that flow through fixed points ${ }^{16}$ in the phase space, which are points that satisfy $\dot{q}=0$ and $\ddot{q}=0$ (so fixed points exist on $p=0$ ). According to equation (3.16), fixed points are the solutions of $\partial_{\phi} f_{F H}+\lambda=0$. Fixed points can be explained with an analogy with moving particles: if a particle has zero velocity (composition gradient) and is not accelerating (rate of change of composition gradient is zero), then it takes an infinite amount of time (film depth) to cross a finite distance (film composition). An infinite amount of film depth is required to pass through the fixed point. As the phase portraits tend to the separatrix, the length of parts of the trajectory passing near the fixed points tend to infinity (imagine a particle passing near a region at which its speed

\footnotetext{
${ }^{15}$ Separatrix: phase portraits that flow through fixed points in phase space. Trajectories that flow through these fixed points (these trajectories must therefore be on the separatrix) correspond to infinitely thick films, hence as (finite) films become thicker their corresponding phase portraits tend to, but don't meet, the separatrix.

${ }^{16}$ Fixed Points: regions of phase space that require an infinite depth of film to pass through, since these points satisfy $\dot{q}=0$ (composition gradient is zero) and $\ddot{q}=0$ (rate of change of composition gradient is zero).
} 

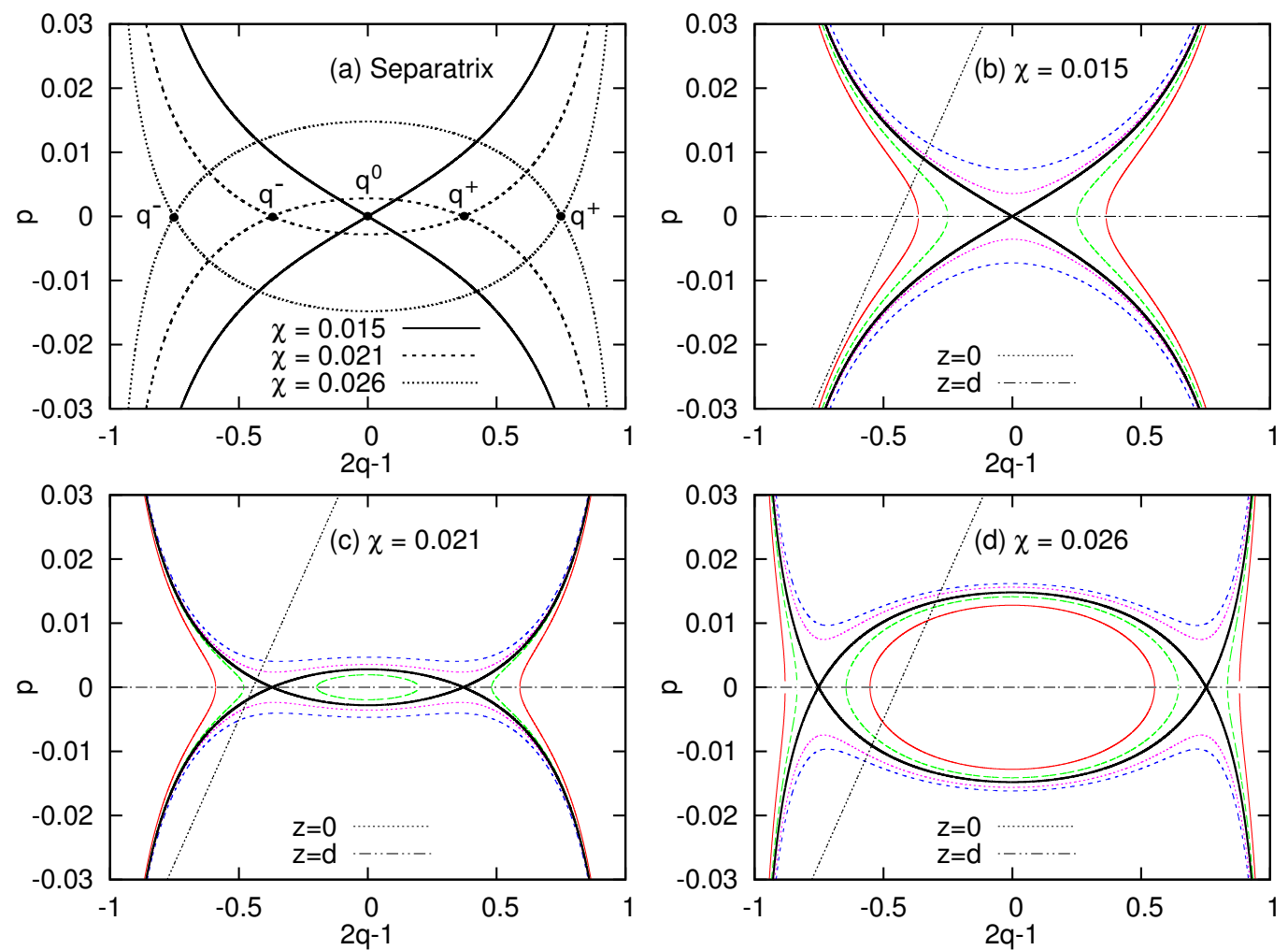

Figure 3.3: Phase portraits for $\lambda=0$ : (a) separatrix only, for all three temperature regimes outlined in the text. The fixed points $q^{-}, q^{0}$, and $q^{+}$are also shown. (b)-(d) examples of phase portraits, including the separatrix, shown for each individual temperature regime. Within each plot (b)-(d), each set of curves with the same colour (dashes/dots) corresponds to the same phase portrait, generated with a single value of $\mathcal{H}$ (see Tables 3.1 and 3.2 for comparable values of $\mathcal{H}$ ). Note that the separatrix and phase portraits are symmetric around $p=0$ and $2 q-1=0$, due to the symmetry of the polymer blend. The boundary conditions arising from the surfaces are plotted in (b)-(d), appearing as straight lines.

drops to zero, thus taking a very long time to pass by this region). Therefore, phase portraits cannot touch the separatrix or pass through the fixed points. The separatrix characterise the general shape of the phase portraits in each temperature regime. The relationship between fixed points, separatrix and temperature regimes is elaborated on in the rest of this subsection.

\section{$\lambda=0$ phase portraits i.e. no material constraints}

I will firstly discuss the separatrix for $\lambda=0$, explaining the relevance of the temperature regimes in terms of where the fixed points are located relative to the boundary conditions. Above the critical bulk temperature, $\chi=0.015$, the separatrix is cross shaped. There is only one fixed point, $q^{0}$, at $(q, p)=(1 / 2,0)$, and the separatrix passes through this point. Solution trajectories will be on the left edge of the flow, since this allows trajectories from the $z=0$ boundary condition to the $z=d$ boundary condition. Below the critical bulk temperature 
but above the wetting temperature, $\chi=0.021$, there are three fixed points, $q^{0}$, $q^{-}$and $q^{+}$, and the separatrix passes through the latter two, which are located symmetrically around $\phi=1 / 2$. Inside those fixed points, the Hamiltonian flow is an orbit around the fixed point $q^{0}$. Note that for the chosen surface energies, the fixed points lie inside the boundary condition at $z=0$, so this boundary condition does not cross the orbital part of the flow, so, again, solution trajectories exist only on the left edge of the flow. Below the wetting temperature, $\chi=0.026$, there are again three fixed points, but the boundary condition at $z=0$ lies inside the leftmost fixed point $q^{-}$, thus crossing the orbital part of the flow twice (this gives an estimation of $\chi_{w}$ [41]; see section 4.3 .3 for more on estimating $\chi_{w}$ ). Because of this, there are several ways in which we can construct a trajectory between the boundary conditions, so in this temperature regime we expect to find coexisting solutions, allowing for lateral phase separation into A-rich and B-rich phases with interfaces perpendicular to the walls, as shown in figure 3.1(b).

\section{$\lambda \neq 0$ phase portraits i.e. with material constraints}

Addressing how $\lambda$ affects the fixed points, and therefore the shape of the separatrix, allows us to characterise how $\lambda$ changes the general shape of the phase portraits. The fixed points are the values of $q$ for which the curve $y=\partial_{q} f_{F H}+\lambda$ crosses $y=0$. Changing $\lambda$ will shift the curve $\partial_{\phi} f_{F H}+\lambda=0$ along the $y$-axis, and move the fixed points to different values of $q$ (along $p=0$ ). This is demonstrated in figure 3.4 for $\chi=0.021>\chi_{c b}$ : above the bulk critical temperature, there are three solutions and therefore three fixed points, as mentioned above for $\lambda=0$ phase portraits. Choosing $\lambda<0$ shifts $q_{\lambda}^{0}<q_{0}$, meaning the separatrix cross-centre or orbit-centre is shifted to lower $q$, whilst both fixed points $q^{-}$and $q^{+}$are shifted to higher $q$. Both of these effects are complimentary, and pull the left side of the phase portraits to the right, which pulls the solution trajectories to higher $q$. For $\lambda>0$ the fixed points shift in the opposite direction. So $\lambda \neq 0$ breaks the symmetry of the phase portraits around $2 q-1$.

\section{Material Constraints}

For a symmetric blend, solutions satisfying the constraints must clearly pass through $q=1 / 2$, else it would not be possible to achieve $\bar{\phi}=1 / 2$. It is clear that many of the trajectories between the boundary conditions in Figure 3.3 do not pass through $q=1 / 2$, and so to obtain valid solutions for a polymer blend between attracting-neutral walls, $\lambda$ must be altered until the solution trajectories pass through $q=1 / 2$, and more specifically until $\bar{\phi}=1 / 2$. Suitable choices of both $\lambda$ and the Hamiltonian constant $\mathcal{H}$ are necessary to produce phase equilibria trajectories of specified depth $d$ and average composition $\bar{\phi}$. This is discussed further in section 3.4.1. 


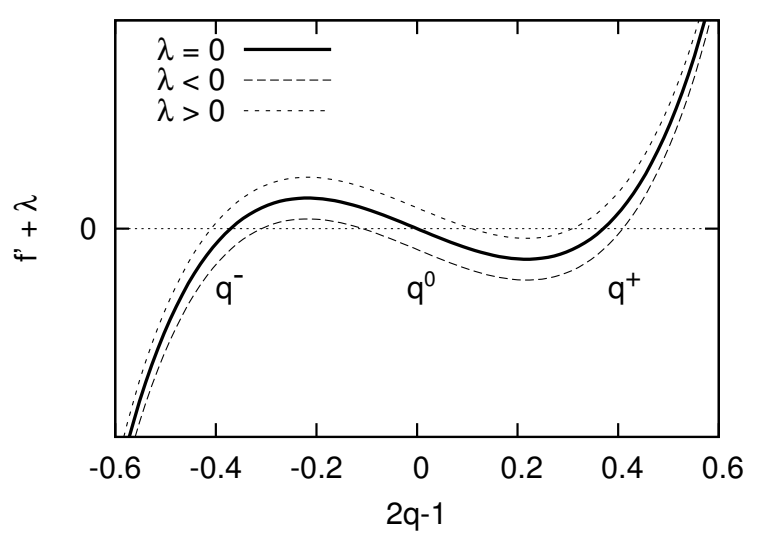

Figure 3.4: Fixed points of the Hamiltonian flow correspond to values of $q$ solving $f^{\prime}+\lambda=0$, where $f^{\prime}=\partial_{q} f_{F H}$. This example is for $\chi=0.021>\chi_{c b}$, so there are three fixed points $q^{-}, q^{0}$, and $q^{+}$. The Lagrange multiplier $\lambda$ shifts the fixed points, as seen by consideration of the curve $f^{\prime}+\lambda$. The fixed points will move along $p=0$ in figures 3.3(a)-(d). Note that the curve $f^{\prime}+\lambda$ for $\chi=0.026$ is similar to the curve presented here, but above the critical bulk temperature $\chi=0.015<\chi_{c b}$ the curve only crosses $f^{\prime}+\lambda=0$ once (for $\lambda=0$ ) at $2 q-1=0$.

\section{Interpretation of $\lambda$}

Solving the Eular-Lagrange equation, Eq. (3.16), subject to constraints (3.14) with $\bar{\phi}=1 / 2$, is equivalent to finding the solution $\phi=\phi^{\prime}$ for which

$$
\left.\delta\left\{\mathcal{F}(\phi(z))+\lambda\left[a^{-1} \int_{0}^{d} \phi(z) d z-\frac{1}{2} d\right]\right\}\right|_{\phi=\phi^{\prime}}=0 .
$$

such that $\phi$ is a general profile and $\phi^{\prime}$ is an equilibrium profile. When $\phi=\phi^{\prime}$ such that $\bar{\phi}=1 / 2$, we see from Eq. (3.26) that

$$
\left.\frac{\delta \mathcal{F}(\phi(z))}{\delta \phi(z)}\right|_{\phi=\phi^{\prime}}=-\lambda a^{-1}
$$

Taking the functional derivative of the free energy (3.1) and assuming $\phi=\phi^{\prime}$ such that Eq. (3.16) is satisfied, we find

$$
\begin{aligned}
\left.a \frac{\delta \mathcal{F}(\phi(z))}{\delta \phi(z)}\right|_{\phi=\phi^{\prime}} & =\left.\left(\partial_{\phi} f_{F H}-\left(\partial_{\phi} \kappa\right) \dot{\phi}^{2}-2 \kappa \ddot{\phi}\right)\right|_{\phi=\phi^{\prime}} \\
& =-\lambda .
\end{aligned}
$$

Finding $\phi=\phi^{\prime}$ subject to the constraint $\bar{\phi}=1 / 2$ requires choosing the correct value of $\lambda$, such that both the Euler-Lagrange equation (3.16) and the constraint equation (3.14) are satisfied. We see, from Eq. (3.27), that the constraint is enforced by $\lambda$ acting as a constant chemical potential across the film $[48,49]$ (the functional derivative $\delta \mathcal{F}[\phi(z)] / \delta \phi$ is proportional to the chemical potential. This is discussed in depth in section 4.3 ). 
Even though the boundary conditions arising from the surface energies do not directly affect the phase portraits, in the sense that the expression for the phase portraits does not contain the surface energies, the effect of the boundary conditions is then not only to pin the value of $\phi$ at the film walls/surface by providing integration limits on Eq. (3.24) (often noted as being the only effect on the solution profile due to focus on symmetric blends confined between antisymmetric walls $[33,37])$ but to effectively apply a field across the film. Of course, the surface energies only act locally at the surfaces, and do not in reality actually apply a field across the film. But the effect of $\lambda$ on the equilibrium profiles (as opposed to the actual, physical effect of the surfaces on the polymer blend) is as if a field is applied across the film (the chemical potential is the conjugate field for $\phi)$. This is demonstrated by the distortion of the symmetric $(\lambda=0)$ phase portraits into asymmetric phase portraits caused by $\lambda \neq 0$.

\subsection{Phase Equilibria of Asymmetric Films}

In this section, I will demonstrate the use of phase portraits for displaying and calculating the phase equilibria of a symmetric binary polymer blend between asymmetrically attracting walls, namely a $B$-attracting wall at $z=0$ (prefers $\phi<1 / 2$ ) and a neutral wall at $z=d$ (no preference on $\phi$ ). The phase portraits are useful for showing how equilibria change as film depth and temperature change. The significance of different temperature regimes will hopefully be more apparent in this section.

In some cases, there are several ways in which we can make a trajectory from the $z=0$ boundary condition to the $z=d$ boundary condition, and in some regimes this allows for coexisting solutions because we can find profiles of the same depth with different trajectories (although these won't have $\bar{\phi}=1 / 2$, but will be rich in one component of the $A-B$ blend). Note that the wall-blend interaction configuration I am using here, namely a $B$-attracting wall at $z=0$ and a neutral wall at $z=d$ is just an example, used because the neutral wall at $z=d$ somewhat simplifies the calculation of the profiles.

In all cases, as the phase portraits approach the separatrix the corresponding profiles are those of thicker films, and when the phase portraits tend to the separatrix the film thickness tends to infinity, such that the equilibria of profiles for phase portraits tending towards the separatrix are representative of the profiles for very thick (tending to infinite) films. The change in the profiles as the thickness tends to infinity can be inferred from the phase portraits, without needing a calculation of the profiles for extremely thick films to be performed.

From this point onward, I will discuss the phase portraits using $q=\phi$ and $p=2 \kappa \nabla \phi(a=1)$. The figures in this section are split into two columns, with the phase portraits on the left and the composition profiles (calculated with equation (3.24)) on the right. The film thickness increases from the top to the bottom subfigure. Both the trajectories and the composition profiles are coloured (shaded) in $\phi$, from black/blue/dark for $\phi=0$ (pure $B$ ) to white/yellow/light for $\phi=1$ (pure $A$ ). This makes comparison of the profiles and the equilibria trajectories slightly easier. 


\section{Numerical Integration}

The integration of equation (3.25) must be done numerically. On the task of integrating between boundary conditions, I noted that rather than select a value of $\mathcal{H}$ and $\lambda$ to generate the phase portraits, it can be simpler to select a value of $\phi_{0}$ and $\lambda$, and from these values calculate a value of $\mathcal{H}$ for which the Hamiltonian flow cross the boundary condition at $\phi_{0}$. This can be done as follows: selecting a value of $\phi_{0}\left(q_{0}\right)$ gives the required value of $\left.2 \kappa \nabla \phi\right|_{0}\left(p_{0}\right)$ from the boundary condition (3.21). Both $q_{0}$ and $p_{0}$ (as well as $\lambda$ ) can then be inserted into equation (3.23), which can then be rearranged to give a value for $\mathcal{H}$. This is sometimes useful because not all values of $\mathcal{H}$ will generate a phase portrait which intersects with the $z=0$ boundary condition, but if a value of $\phi_{0}$ (the value at which the boundary condition crosses the flow) is chosen first, this problem is avoided (though it does not guarantee that the flow will also intersect with the $z=d$ boundary condition).

\subsubsection{Independently-existing profiles}

Independently-existing solutions, such as that in figure 3.1(a), must have $\bar{\phi}=1 / 2$ in order to conserve material for a symmetric polymer blend, $\mathcal{H}$ and $\lambda$ having been adjusted for this requirement, these values being shown in order of figure appearence in table 3.1. We can calculate such a profile for all temperature regimes, even if it is not the most energetically favourable profile (this is discussed in section 3.4.2).

\section{Above critical bulk temperature: $\chi=0.015$}

We see from figure 3.3(b) that the boundary conditions cross the $B$-rich (leftmost) part of the flow when $\lambda=0$. Setting $\lambda<0$ pulls this part of the flow past $\phi=1 / 2$,

\begin{tabular}{ccc}
\hline \hline$\chi$ & $\lambda$ & $\mathcal{H}$ \\
\hline 0.015 & -0.001636 & 0.0040824 \\
& -0.001387 & 0.003942 \\
& -0.000781 & 0.0035997 \\
& -0.000537 & 0.00346405 \\
& -0.000330 & 0.003351899 \\
\hline 0.021 & -0.001387 & 0.0024925 \\
& -0.000281 & 0.0018621 \\
& -0.000104 & 0.0017598 \\
& -0.000037 & 0.001721 \\
& -0.000006 & 0.001703 \\
\hline 0.026 & -0.001144 & 0.0011826 \\
& -0.000829 & 0.001020 \\
& -0.000089 & 0.0007045 \\
& +0.000120 & 0.0007585 \\
& +0.000058 & 0.0008682 \\
\hline
\end{tabular}

Table 3.1: $\lambda$ and $\mathcal{H}$ for the independently-existing profiles shown in figures 3.5, 3.6 and 3.7. The order of rows corresponds to the order of appearence of the sub-figures. 

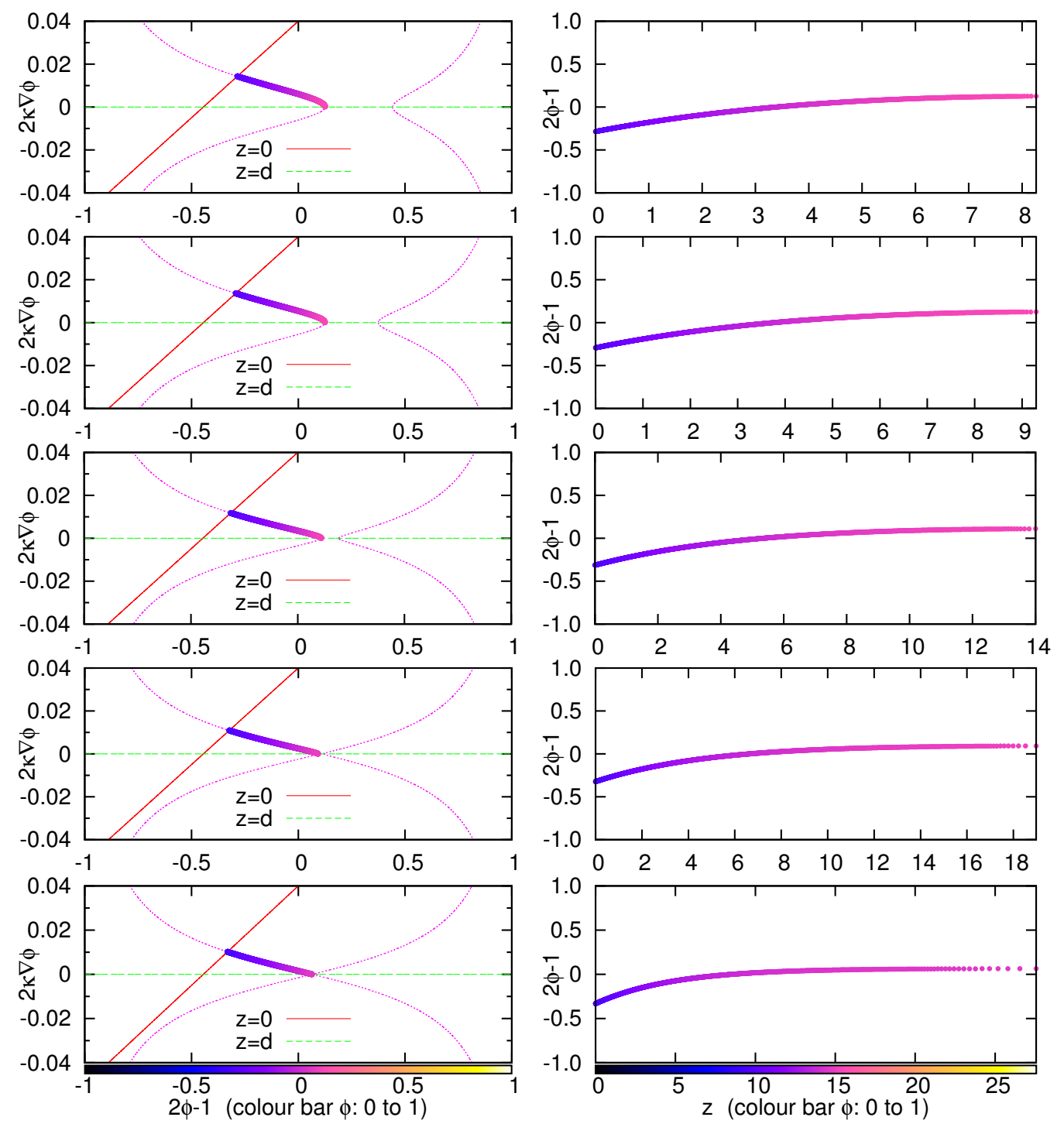

Figure 3.5: Phase portraits (left) and profiles (right) for a symmetric blend, $\chi=$ $0.015<\chi_{c b}$ (one-phase region); increasing film depth from top to bottom. The bold (coloured) line shows the trajectory that satisfies the boundary conditions. There is an excess of $B$ and a gradient at $z=0$ (the $B$-attracting wall). As $z \rightarrow d$, the gradient approaches zero and the profile becomes constant with an excess of $A$. These profiles are a monolayer with an excess of $B$ at the $B$-attracting wall, since in this temperature the polymer blend is miscible (this means there is little change in the profiles as film depth increases). 
and suitable tuning of $\lambda$ and $\mathcal{H}$ ensures material conservation $\bar{\phi}=1 / 2$ for the profiles. Example phase portraits are shown in figure 3.5. The bold line shows the solution trajectory that satisfies both of the boundary conditions and $\bar{\phi}=1 / 2$. There is an excess of $B$ and a composition gradient at $z=0$, due to this surface preferring the $B$-component.

As film depth increases, the phase portraits show that the excess of $\mathrm{B}$ at $z=0$ increases whilst the gradient at that wall, and throughout the film, decreases. This is a consequence of there being more bulk material to draw B-component from, and a longer distance over which the profile can relax from the wall effects. As $z \rightarrow d$ (the film thickness), the gradient approaches zero and the profile becomes constant with a slight excess of $A$-component, due to the neutral surface at $z=d$ enforcing $\left.\nabla \phi\right|_{d}=0$ and depletion of $A$ elsewhere in the film, respectively. The profiles are all a monolayer ${ }^{17}$ with approximately $\phi=1 / 2$ but an excess of $B$ at the $B$-attracting wall. This is because $\chi<\chi_{c b}$ and so the polymer blend is miscible (the bulk fluid would be in the one-phase region).

Below critical bulk temperature, above wetting temperature; $\chi=0.021$

There are three fixed points in this temperature regime, as demonstrated in figure 3.4. Example phase portraits are shown in figure 3.6. The phase portraits approach the fixed point $q^{+}>q^{0}>1 / 2$ as $d \rightarrow \infty$, developing a turning point near the $z=0$ boundary condition as the trajectories circumvent the fixed point $q^{0}$, which is now orbital (the trajectories cannot tend towards this point). This turning point means that, although the profile is monotonic, there is a region slightly displaced from the $B$-attracting wall at which the rate of change of $B$ towards that wall decreases and the profile becomes less steep. Very close and up to this wall, the rate of change of $B$ then accelerates towards the wall again. The excess of $B$ near the $B$-attracting wall extends deeper into the film for $\chi>\chi_{c b}$ than for $\chi<\chi_{c b}$, since the profiles are now a bilayer, since $\chi>\chi_{c b}$ (the bulk fluid would be in the two-phase region).

Bilayer $^{18}$ means that the film effectively consists of two layers, each rich in a different component of the blend and each coating a different wall, in this case the $B$-rich ( $A$-rich) layer coating the $B$-attracting (neutral) wall. In the case of an antisymmetric wall regime (which would be achieved by making the $z=d$ wall $A$-attracting in the same way that the $z=0$ wall is $B$-attracting) on decreasing temperature from $\chi<\chi_{c b}$ to $\chi>\chi_{c b}$ there is a soft-mode transition from a monolayer to a bilayer, in which an $A(B)$-rich phase exists between the $A(B)$ attracting wall and the centre of the film, with a soft interface centred on $\phi=1 / 2$. There is an analogous transition for asymmetric films.

The blend is still relatively miscible, since $\chi$ is still fairly close to $\chi_{c b}$, and so the interface in the bilayer is still fairly diffuse, although for thicker films the interface is narrow compared to the layers. This can be demonstrated for

\footnotetext{
${ }^{17}$ Monolayer: the profile is approximately constant $\phi(z) \approx \bar{\phi}$ since the blend is miscible. There may be an excess of a component near the film surfaces due to preferential attraction, depleting that component in the rest of the film.

${ }^{18}$ Bilayer: the film is vertically segregated into two phases (layers) with an interface between them. These phases coat the film surfaces due to preferential surface attraction.
} 

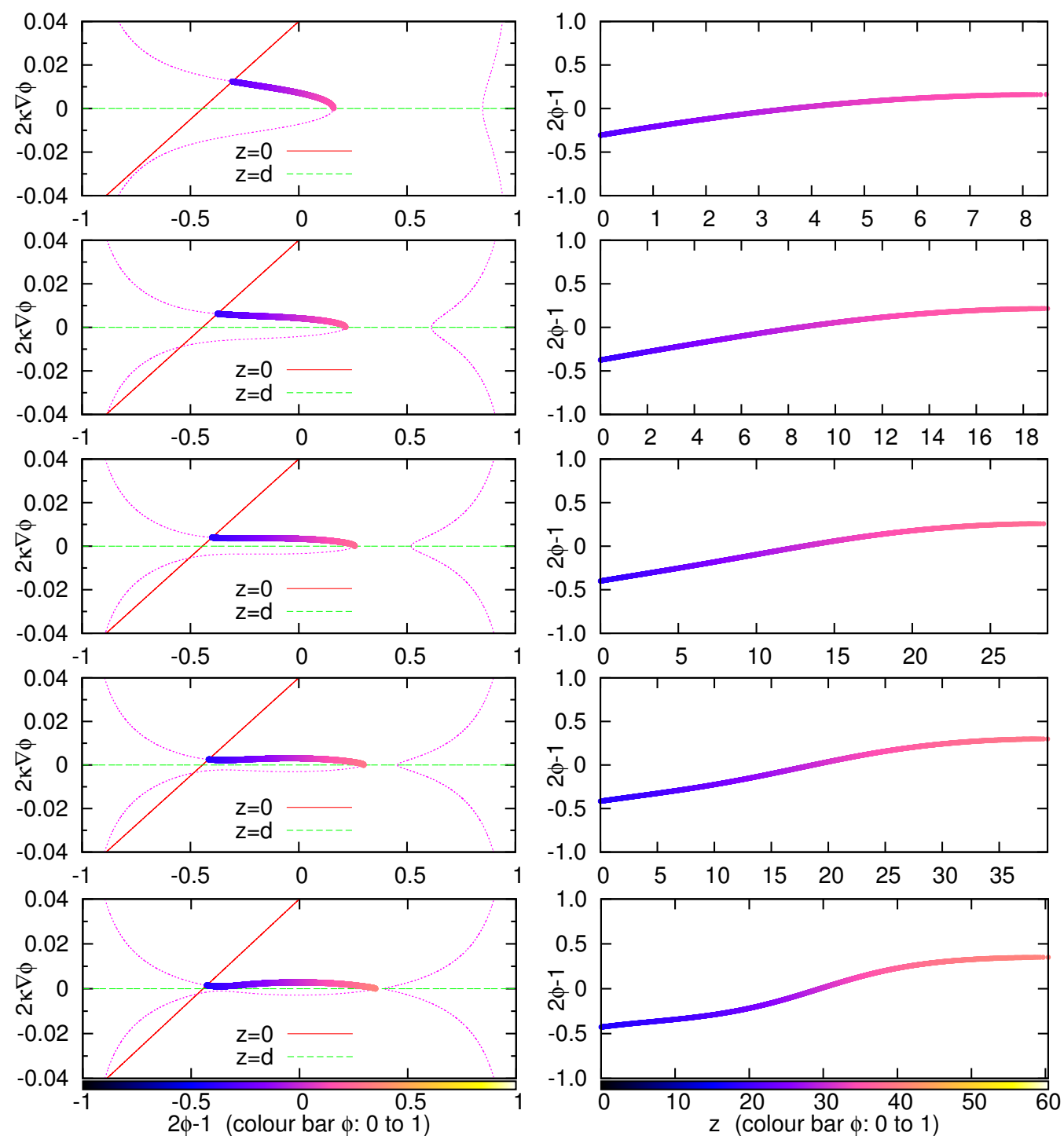

Figure 3.6: Phase portraits (left) and profiles (right) for a symmetric blend, $\chi=0.021>\chi_{c b}$; increasing film depth from top to bottom. The bold line shows the trajectory that satisfies the boundary conditions. As the phase portraits tend towards the separatrix for thicker films, a minimum appears in the phase portraits close to the $z=0$ boundary condition, meaning that very near the $B$-attracting wall the richness of $B$ in the profile accelerates towards the wall, but there is a region slightly displaced from this wall where the steepness of the profile becomes gentler as the trajectories near $2 \kappa \nabla \phi=0$. The profiles are a bilayer since $\chi>\chi_{c b}$, although the blend is still rather miscible due to the shallow distance into the two-phase region, so the interface is still rather diffuse, hence thicker films are required to find a more pronounced bilayer. 

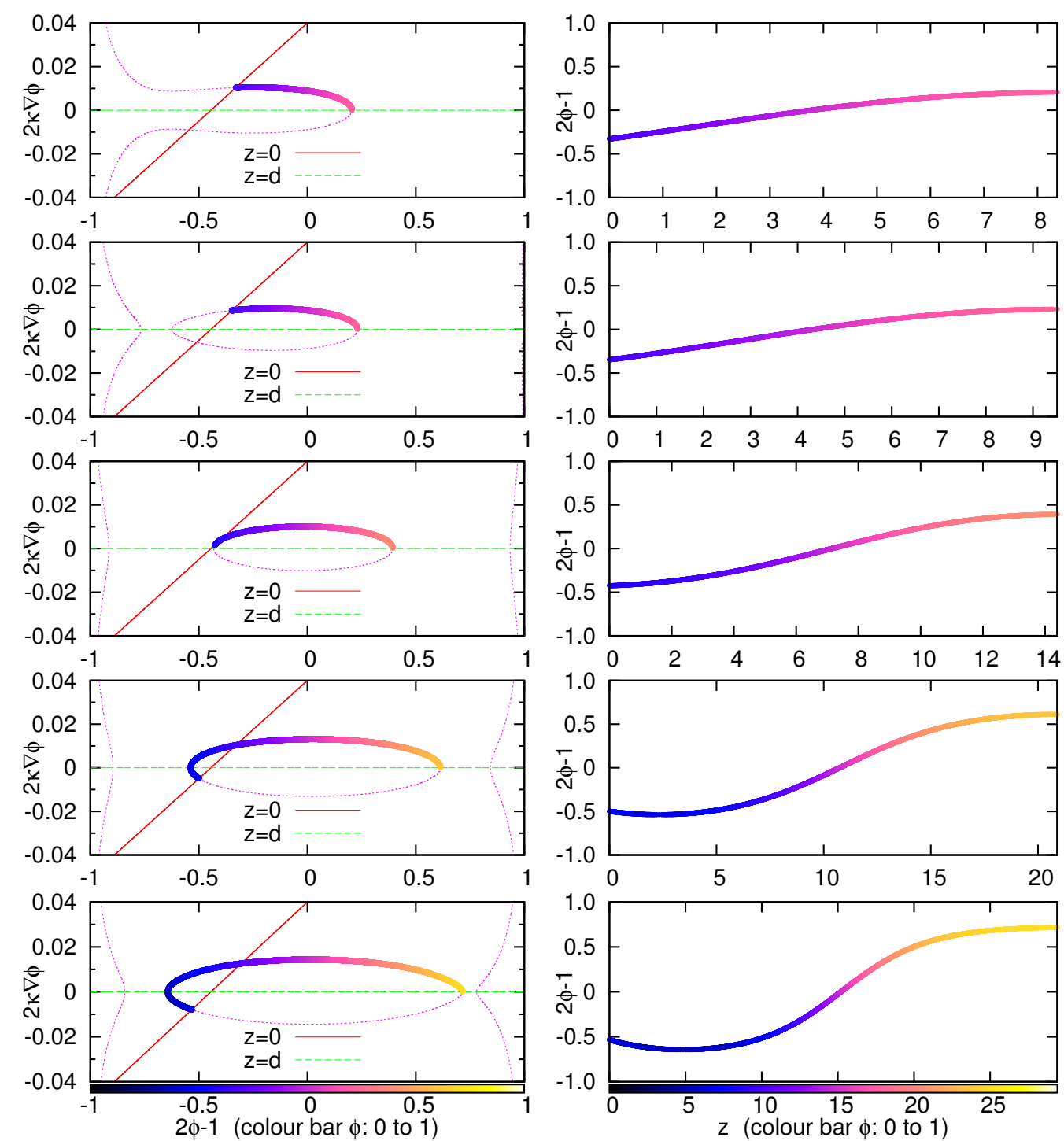

Figure 3.7: Phase portraits (left) and profiles (right) for a symmetric blend, $\chi=0.026>\chi_{w}$; increasing film depth from top to bottom. The bold line shows the trajectory that satisfies the boundary conditions. The boundary conditions cross the orbital part of the Hamiltonian flow for thick enough films. Above a certain depth, the trajectories at $z=0$ fall below $2 \kappa \nabla \phi<0$, because the trajectory begins at the first cross of the flow with the $z=0$ boundary condition (this is required to find trajectories corresponding to deeper films). This means that the profiles are no longer monotonic, and the profiles are richer in $B$ at a point near the $B$-attracting wall than at the $B$-attracting wall. For thicker films, the latter $B$-rich region becomes thicker as the phase portraits tend towards the $q^{-}$fixed point. The bilayer nature of the film is quite pronounced, as the temperature is further from the bulk critical temperature than for $\chi=0.021$. 
films with $d \rightarrow \infty$ from the phase portraits: the $B$-rich and $A$-rich parts of the flow approach $q^{-}$and $q^{+}$respectively as the films become thicker, and the layers corresponding to these regions become infinitely thick in the limit $d \rightarrow \infty$, whilst the interface corresponds to the bump in the trajectory around $\phi=1 / 2$, and since this part of the trajectory cannot approach the fixed point $q^{0}$, the interface will tend to a finite width in the limit $d \rightarrow \infty$.

\section{Below wetting temperature; $\chi>\chi_{w}$}

Example phase portraits are shown in figure 3.7. The phase portraits are qualitatively similar to those for $\chi=0.021$, the main difference being that the boundary conditions now cross the orbital part of the Hamiltonian flow, so solution trajectories pass through $\phi=1 / 2$ even for $\lambda=0$ (non-distorted phase portraits, figure $3.3(\mathrm{~d})$ ). For films above a characteristic depth, the trajectories cross the $z=0$ boundary condition for $2 \kappa \nabla \phi<0$, so the profiles are no longer monotonic: the profiles have an inflexion point in $\phi$ near the B-attracting wall, so there is an initial increase in B-component on moving away from the B-attracting wall. The bilayer is far more pronounced than for $\chi=0.021$, since the blend is more immiscible for $\chi=0.026$. As $d \rightarrow \infty$, the $B$-rich turning point in the profile trajectory will approach the $q^{-}$turning point and this region of the $B$-rich layer will become infinitely thick. Similarly, the $\nabla \phi \approx 0$ region of the $A$-rich layer will become infinitely thick as trajectories approach the $q^{+}$fixed point. The interface width will tend to a finite value in the limit $d \rightarrow \infty$, since the part of the trajectory constituting the interface cannot approach the $q^{0}$ fixed point. Note that these independently-existing solutions are not the lowest free energy solutions available, and lateral phase separation into coexisting equilibria is favourable.

\subsubsection{Coexisting solutions}

Below the wetting temperature $\chi>\chi_{w}$, a single solution with $\bar{\phi}=1 / 2$ is not energetically favourable, and a lower energy configuration for the film can be obtained by having two coexisting solutions, one rich in $A\left(\bar{\phi}_{A}>1 / 2\right)$ and the other rich in $B\left(\bar{\phi}_{B}<1 / 2\right)$. This is really the very definition of the wetting temperature: the cut-off temperature at which an independently-existing solution, in which layers 'wet' the surfaces as in figure 3.1(a), is not energetically preferable to coexisting solutions, in which two phases are in contact with each surface as in figure 3.1(b). Calculation of the free energy can be done using equation 3.1 for the calculated profiles. Wetting is discussed in section 2.2 and figure 2.2. These coexisting phases together conserve the average composition of the blend by adjustment of the area of the film occupied by each phase, and by the richness of each phase in either component.

\section{Forming a tractable problem}

Presently I consider $\lambda=0$, leaving a generalisation to $\lambda \neq 0$ for the discussion in Sec. 3.6. The reason for this is as follows. The coexisting solutions in a real film will need to have $\lambda_{A}=\lambda_{B}$ i.e. the chemical potential of coexisting equilibria 


\begin{tabular}{ccc}
\hline \hline$\chi$ & $\lambda$ & $\mathcal{H}$ \\
\hline 0.026 A-rich & 0 & 0.00080 \\
& 0 & 0.00090 \\
& 0 & 0.0009235 \\
\hline 0.026 B-rich & 0 & 0.00089 \\
& 0 & 0.00092 \\
& 0 & 0.00092384 \\
\hline
\end{tabular}

Table 3.2: $\lambda$ and $\mathcal{H}$ for the coexisting profiles shown in figures 3.8 and 3.9. The order of rows corresponds to the order of appearence of the sub-figures.

must be the same at equilibrium. However, trying to find an $A$-rich and a $B$ rich solution of the same depth, with the same chemical potential $\lambda_{A}=\lambda_{B} \neq 0$, such that at this chemical potential the pairs of profiles have a lower free energy than the profiles with a different chemical potential, is an intractable problem (and, furthermore, we still would not be taking into account the lateral interface between the laterally coexisting phases in this $1 \mathrm{D}$ consideration). In order to make the problem of finding coexisting phases tractable, it is simplest to restrict to $\lambda=0$, since we guarantee $\lambda_{A}=\lambda_{B}$ and each coexisting phase will have a lower free energy than for any $\lambda \neq 0 . \quad \lambda \neq 0$ means that the free energy of solutions is greater than for the 'same' solutions with $\lambda=0$ ('same' is meant in the sense that the depth is the same, and the trajectory is qualitatively similar except for the distortion of the phase portraits away from symmetry by non-zero $\lambda$ ) because the lowest energy solutions are found subject to no constraints at all.

With $\lambda=0$ and $\chi=0.026$, it can be seen from the phase portraits of figure $3.3(\mathrm{~d})$ that there are several different trajectories between the boundary conditions available, since each boundary condition crosses the flow more than once (in a region where a valid trajectory between the boundary conditions can be made). With $\lambda=0$ these solutions all correspond to $\bar{\phi} \neq 1 / 2$, and depending on the trajectory will either be $A$-rich $(\bar{\phi}>1 / 2)$ or $B$-rich $(\bar{\phi}<1 / 2)$.

\section{Coexisting Phase Equilibria}

The values of $\mathcal{H}$ used to generate figures 3.8 and 3.9 are given in table 3.2. A-rich profiles, shown in figure 3.8, can be found for all depths above a minimum depth $D$, therefore $A$-rich solutions must have a minimum length $D$. The reason for this minimum depth can be inferred from the phase portraits: it is not possible to adjust the phase portraits via $\mathcal{H}$ such that the crosses of the flow with the $z=0$ and $z=d$ boundary conditions come arbitrarily close together (the orbital part of the flow, upon 'shrinking' along the $x$-axis direction, would no longer cross the $z=0$ boundary condition), therefore there is a limit on how small $A$-rich solutions can become for $\lambda=0$. The $A$-rich solutions have a high $B$ excess near the attracting wall, but quickly approach a constant $A$-rich composition away from this wall. The excess of $A$ at the neutral wall is due to depletion of $B$ in the film by the $B$-attracting wall.

$B$-rich profiles can be found all depths, as can be inferred from figure 3.9, because the crosses of the boundary conditions with the flow for $z=0$ and $z=d$ 

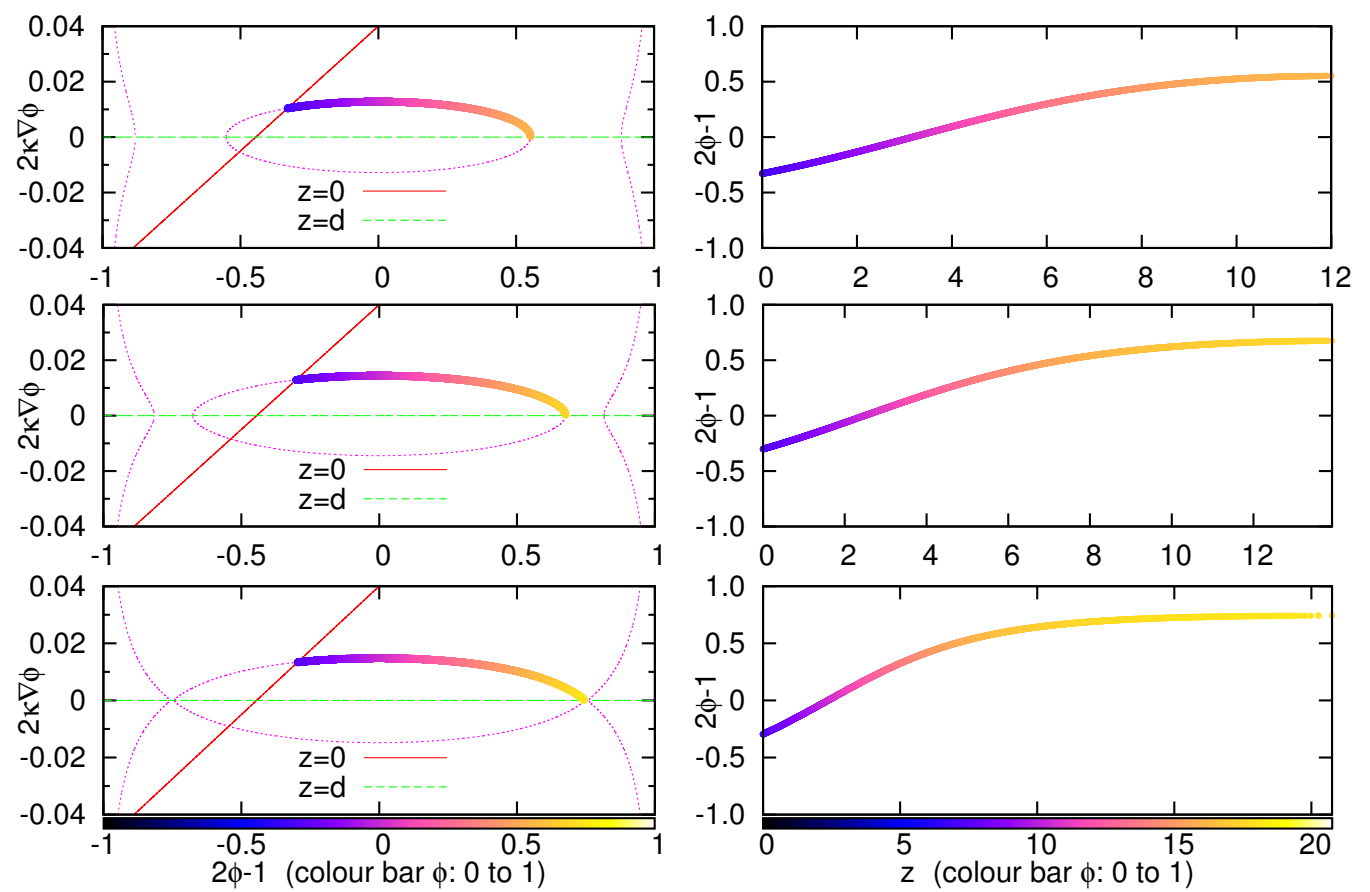

Figure 3.8: For $\chi=0.026$ and $\lambda=0$, phase portraits (left) and profiles (right) for $A$-rich profiles. The phase portraits are symmetrical since $\lambda=0$ (the blend is symmetric). Longer $A$-rich solutions are all similar, beginning on the rightmost cross of the $z=0$ boundary condition with the flow. $d \rightarrow \infty$ as the phase portraits approach the separatrix, the thickness of the $A$-rich part of the profile, for which $\nabla \phi \approx 0$, tends to infinity. There is an excess of $B$ at $z=0$.

can be brought together to allow $d \rightarrow 0$. The $B$-rich profiles are approximately constant and very rich in $B$, but a drop in $B$ occurs close to the $B$-attracting wall; it appears to be unfavourable for the $B$-attracting wall to adsorp (excess attracted by surface) material with the same richness of $B$ found in the bulk.

Imposing that $A$-rich and $B$-rich solutions must have equal depths, we must infer that films with depths less than $D$ cannot undergo lateral phase separation and must exist in a single state with $\bar{\phi}=1 / 2$, because an $A$-rich solution does not exist to allow lateral phase separation. For depths greater than $D$, lateral phase separation is energetically favourable, because the energy of both of these coexisting equilibria is less than the energy of a $\phi=1 / 2$ independently-existing equilibria, and material conservation can be accounted for by varying the area of these laterally segregated phases in the film. This result is an analogue of the result for blends between antisymmetric walls for $\chi>\chi_{w}$, for which lateral phase separation can occur only for films above a minimum depth [40, 41].

\subsection{Surface Roughening}

Figure 3.10 is a plot of the free energy, calculated with equation (3.1), of laterally coexisting profiles calculated for $\lambda=0$, for profiles with a range of film depths $d$ (the free energy is calculated from equation (3.1)). The free energy decreases with 

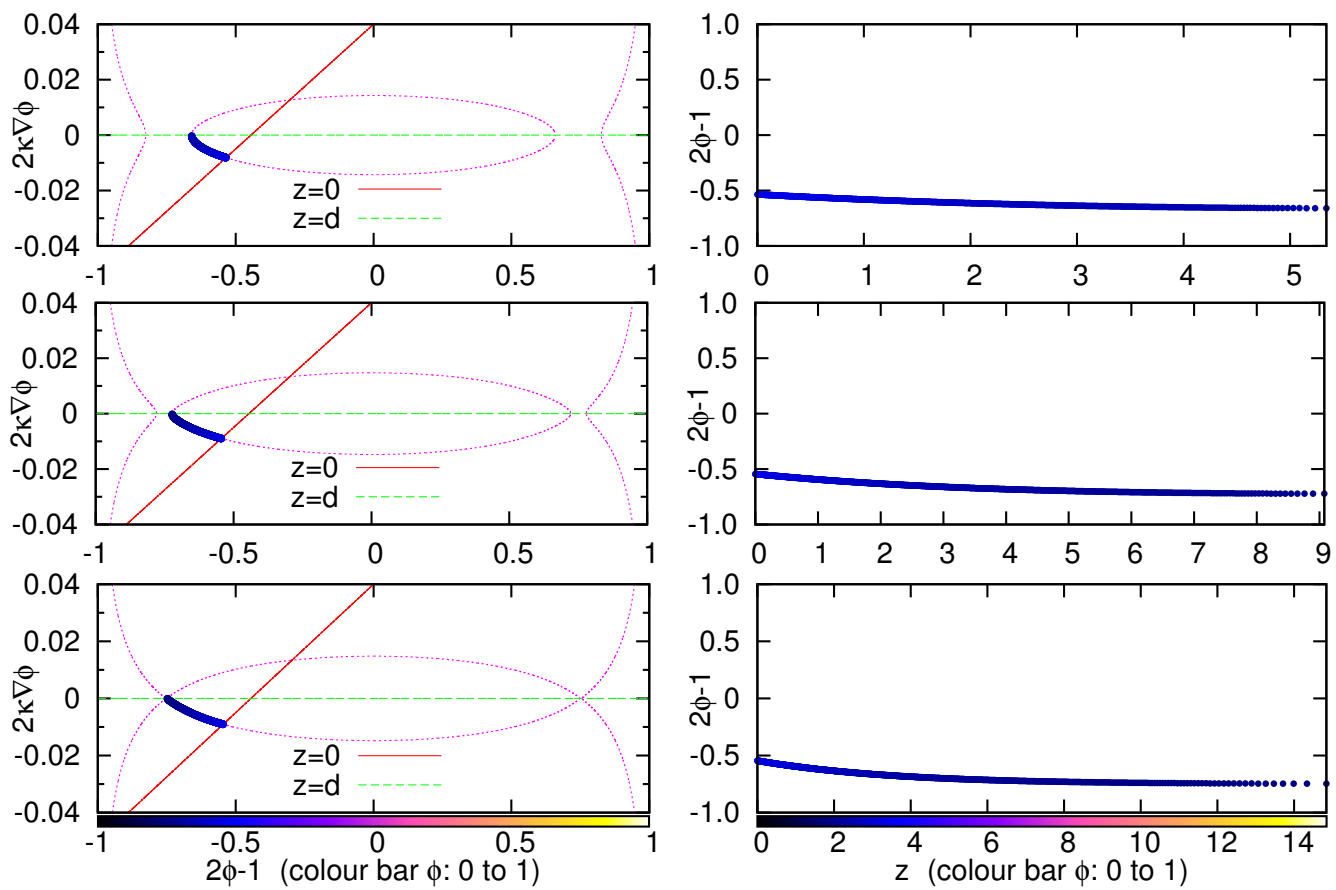

Figure 3.9: For $\chi=0.026$ and $\lambda=0$, phase portraits (left) and profiles (right) for $B$-rich profiles. The phase portraits are all symmetrical since $\lambda=0$ and the blend is symmetric. Longer $B$-rich solutions are all qualitatively similar and exist for all possible film depths. The profiles are nearly constant, but the $B$-attracting wall at $z=0$ is less $B$-rich than both the bulk and the neutral wall at $z=d$.

the thickness of the film as a consequence of the composition gradients becoming less steep as the films become thicker. Imposing that $\lambda=0$, consider films (before lateral phase separation occurs) of depth slightly below $D$, the minimum depth of $A$-rich profiles. In this case, neglecting the effects of gravity and other kinetic considerations, consideration of the free energy shows it is thermodynamically favourable for the film to laterally phase separate into an $A$-rich phase with $d_{A} \geq D>d$ and a $B$-rich phase with $d_{B}<d<D$, since these phases still have less energy than an independently-existing profile.

If one of the walls is a free surface then there is no need to impose that the depths of the laterally separated phases are equal, so the $A$-rich phase will protrude from the $B$-rich phase, since $d_{A}>d_{B}$. This is because the $A$-rich phase has the greater free energy per unit area (for $\lambda=0$ ) than the $B$-rich phase, as demonstrated by figure 3.10 , so we might expect the $A$-rich phase to be deeper and richer in $A$, since this accounts for material conservation whilst minimising the area of film occupied by the $A$-rich phase (if the phase with the higher free energy per unit area occupies less area by increasing in height, we would expect the free energy to reduce). In this case, the difference in depths arises so that an A-rich phase may exist for $\lambda=0$, allowing lateral phase separation. However, for films with initial depth $d>D$, we may expect the same phenomena as a result of lateral phase separation, again with the $A$-rich phase protruding from the film, since the $A$-rich phase still has a lower free energy than the $B$-rich phase. Figure 


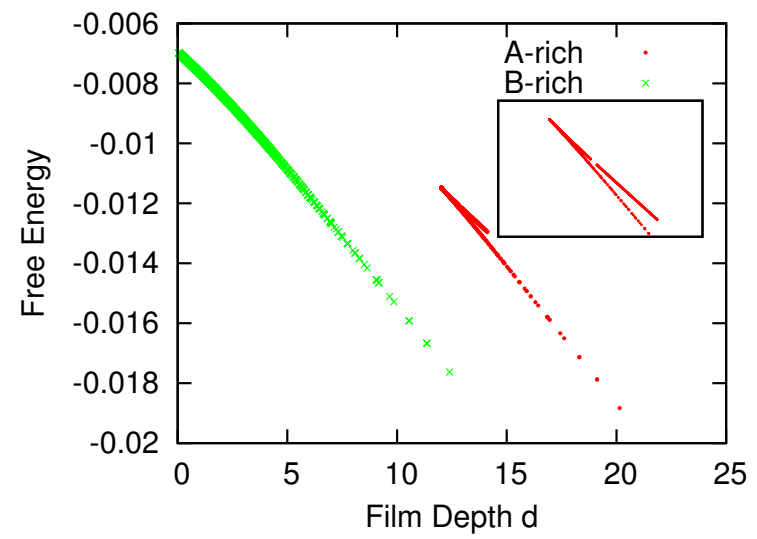

Figure 3.10: Free energy of the $A$-rich and $B$-rich solutions for $\lambda=0$ and $\chi=$ 0.026. Both solutions can go to arbitrarily high depths, but there is a lower limit on the depth for the $A$-rich solution (not on the $B$-rich solution). Of interest for the $A$-rich solution is that there is a region of two solutions for the same height, which is a result of being able to calculate an $A$-rich solution from the leftmost cross of the orbital flow with the $z=0$ boundary condition, rather than just the rightmost cross (although the former yields a higher energy profile; the upper branch results from calculating the profile from the leftmost cross). The $A$-rich phase has a higher free energy than the $B$-rich phase.

3.11 is a schematic of this scenario.

However, there is no need to enforce $\lambda=0$, unlike for antisymmetric films. Allowing $\lambda \neq 0$, with the understanding that the heights of coexisting solutions may differ, the role of $\lambda$ changes subtly, since now it can act to alter the depth of the equilibria, as well as the average volume fraction. Changing $\lambda$ from zero whereby a depth $d$ is obtained, to $\lambda^{\prime}$ whereby a depth $d^{\prime}>d$ is obtained, can have the effect of decreasing the free energy, although the energy will be greater than that for a solution of depth $d^{\prime}$ for $\lambda=0$.

We then expect in general that, for a laterally phase separated film with a depth greater than $D$, it is favourable for the $A$-rich and $B$-rich solutions to exist at different heights. With $\lambda \neq 0$, there can be a distortion of the phase space to obtain $A$-rich solutions with $d_{A}<D$, so that the minimum depth of $A$-rich solutions is certainly below $D$. There will be a compromise between the average volume fraction $\bar{\phi}_{A}$ and $\bar{\phi}_{B}$, the depths $d_{A}$ and $d_{B}$, the total area of the $A$-rich and $B$-rich phases, and $\lambda_{A}$ and $\lambda_{B}\left(\lambda_{A}=\lambda_{B}\right.$ at equilibrium), to obtain two solutions which may coexist together in order to produce the lowest free energy overall. From a purely thermodynamic argument, without any particular symmetries of the confining walls/surfaces, in general we should not expect the coexisting solutions to have equal depths, and that a scenario like that in figure 3.11(b) may be rather general. 


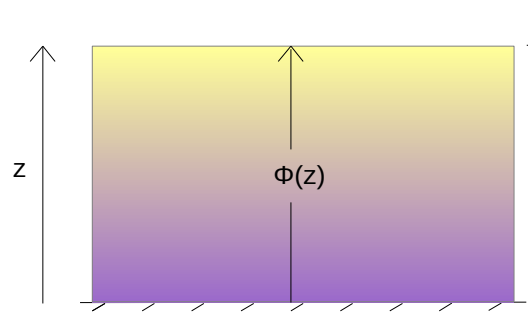

(a)

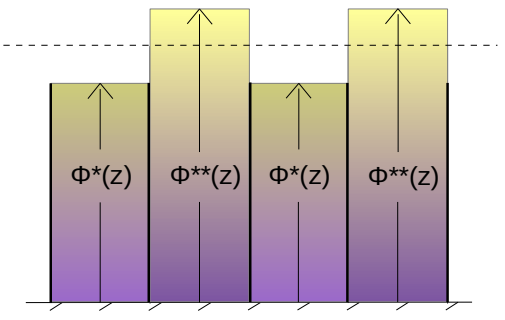

(b)

Figure 3.11: Schematic of a binary polymer-blend film. (a): film with a free top surface, with a single profile $\phi(z)$ describing the entire film; (b): coexisting equilibria $\phi^{*}(z)$ and $\phi^{* *}(z)$ which exist side-by-side, but at different heights, since this provides a lower energy configuration. The lateral interfaces between the coexisting phases are not taken into account.

\subsection{Summary}

I have described how the phase equilibria of a binary polymer-blend thin film between selectively attracting walls can be studied with Hamiltonian Phase Portraits. This method was originally developed and used by Souche and Clarke for the special case of a symmetric blend between antisymmetric walls [41]. I extended the method to include a Lagrange multiplier, allowing this method to be generalised to asymmetric films. This chapter was restricted to a specific asymmetry, although this can be generalised to general surface asymmetry and to asymmetric blends. Hamiltonian Phase Portraits can be used to visualise and calculate phase equilibria in the 'depth dimension', but qualitative conclusions can be drawn about the nature of laterally coexisting phases that can exist in a laterally segregated film. This method treats coexisting phases as if they were effectively isolated from each other, since I have not considered the interfaces between the coexisting phases or the implications of surface tension and surface connectivity. However, from a purely thermodynamic perspective, surface roughening whereby one phase is deeper/higher than the other should be a general mechanism by which a film can lower its free energy upon lateral phase separation.

The next chapter uses Hamiltonian Phase Portraits to explain the results of 2D simulations of polymer-blend thin films, showing how knowledge of 1D equilibria (calculated only in the dimension extending from one film surface to the other, as in this chapter) is sufficient to explain the kinetics of phase separation. 


\section{4}

\section{LATERAL Phase Separation \\ VIA \\ SuRFACE Bifurcation}

I use diffusion simulations of polymer blend films with selectively attracting surfaces to identify the dynamics of lateral phase separation via a transient wetting layer, utilising Hamiltonian phase portraits of 1D phase equilibria to explain a surface bifurcation mechanism. This mechanism describes how the surface values of the equilibrium profile of a transient wetting layer, which are effectively pinned to boundary conditions at the film surfaces, divide into two values for laterally coexisting equilibria. The requirement that these effective boundary conditions be satisfied at all times, not only at equilibrium, causes the particular dynamics of the breakup of the transient wetting layer. This work was published in my second paper (Letter) "Breakup of a Transient Wetting Layer in Polymer Blend Thin Films: Unification with 1D Phase Equilibria" [50] and third paper "Lateral phase separation in polymer-blend thin films: Surface bifurcation" [51].

4.1 Introduction . . . . . . . . . . . . . . . . . . . . 72

4.2 Calculating phase equilibria in 1D . . . . . . . . . . . . 75

4.3 Modelling Phase Separation . . . . . . . . . . . . . . . . . . 77

4.4 1D equilibria in 2D films . . . . . . . . . . . . . . . . 83

4.5 Breakup of a Transient Wetting Layer . . . . . . . . . . . . . . . . . . 90

4.6 Discussion . . . . . . . . . . . . . . . . . . . . . . . . . . . 102

4.7 Summary . . . . . . . . . . . . . . . . . . . . . . . . 104 


\subsection{Introduction}

In this chapter I develop a diffusion equation for binary polymer-blend thin films with selectively attracting surfaces. Included in this equation are often neglected surface terms that are required so that the equilibria that appear in the simulations match those that can be calculated using, for example, the Hamiltonian Phase Portraits method presented in chapter 3. I then perform simulations with this equation for various different temperatures, depths and surface attraction regimes. I show that the simulation results are fully consistent with the calculated phase equilibria, and show how the coexisting equilibria that can be calculated in $1 \mathrm{D}$ using phase portraits do appear in the (2D) simulations, albeit under a nonzero chemical potential due to the lateral interfaces between the phases (which cannot be accounted for with the 1D Hamiltonian Phase Portrait method). I also explain how effective boundary conditions at the film surfaces serve to pin the profiles of the films to particular values, even out of equilibrium.

I use these simulations to identify the dynamics of lateral phase separation via a transient wetting layer, which is the process by which a film initially vertically segregates and then undergoes lateral phase separation into laterally coexisting equilibria (which is favourable below the wetting temperature). Utilising Hamiltonian phase portraits of the $1 \mathrm{D}$ phase equilibria that appear in the simulations, I explain a surface bifurcation mechanism. This mechanism describes the particular way in which the surface values of the transient wetting layer divide into two values which evolve towards those of the laterally coexisting equilibria, but in such a way that the surface boundary conditions are still honoured, and how this is ultimately responsible for the particular dynamics of the breakup of the transient wetting layer.

\subsubsection{The importance of dynamics and kinetics}

The difference between the wetting regime, in which one phase completely covers a surface and excludes another phase from contact with that surface, and the nonwet regime, in which both phases are in contact with a surface, was discussed throughout chapter 2. Figure 2.2 shows a binary blend in contact with a planar surface: when the contact angle that a phase makes with the surface goes to zero, the surface is wet by that phase. However, the discussions in chapter 2, and the literature cited therein, primarily concerned describing wetting transitions in the context of wetting layers which become infinitely thick in a semi- $\infty$ geometry, although there was a discussion of films of finite thickness which discussed how equilibrium in the non-wetting regime is laterally coexisting phases which are both in contact with the surface.

Of equal importance are the kinetics by which a surface may wet or de-wet. A film of an initially homogeneous blend does not spontaneously adopt an equilibrium profile, and the pathway by which the films evolve are very important for determining the final morphology, particularly for polymer-blend films, in which the morphology can become 'frozen' far away from equilibrium (for example, when a solvent which keeps the blend a fluid is all removed; see description of 
spin coating process below). Similarly, the final morphology may not necessarily be that predicted by consideration of the lowest free energy equilibria; it may be another equilibrium profile (a metastable equilibrium) that was reached first due to the kinetics of the system.

Early numerical studies of binary blends made the distinction between the layered morphology of Vertical Phase Separation ${ }^{19}$ (one phase in contact with a surface; see figure 4.1(a)) and the non-wet morphology of Lateral Phase Separation $^{20}$ (both phases in contact with a surface; see figure 4.1(b)): a high surface field, low thermal noise regime corresponded to a layered morphology, and a low surface field, high thermal noise regime corresponded to a partially wet morphology [52]. The competition between surface attraction of particular blend components and thermal noise that will generally move the blend towards a lower free energy (which may not be a layered morphology with only one phase in contact with each surface) highlights the importance of kinetics in determining the morphology of the film: vertical layering may initially occur, but is not necessarily stable. The vertical layering of phases was realised both experimentally [53] and computationally $[54,55]$, even in the non-wet regime when it is energetically favourable for both phases to be in contact with the surface $[56,57]$ i.e. vertical phase separation into layers (like in figure 4.1(a); alternating layers extending into the film are also possible) occurred even when lateral phase separation (like in figure 4.1(b)) was energetically favourable. Experiments later revealed that the vertical layers forming in the non-wet regime can break up as lateral structures appear at a surface [58], with solvent evaporation experiments allowing frozen out-of-equilibrium states to be studied to investigate this breakup [59].

\subsubsection{Lateral Phase Separation via a Transient Wetting Layer}

The main subject of this chapter is the lateral phase separation of an initially vertically segregated ${ }^{21}$ film resulting in a laterally segregated ${ }^{22}$ film. The pioneering work of Walheim highlighted the possibility of lateral phase separation via a transient wetting layer ${ }^{23}$ [59]: the initial formation of a transient (temporary) wetting layer that forms as the blend initially vertically phase separates, due to preferential attraction by the film surface(s), which subsequently breaks up due to an instability. This subject has received more recent attention [60, 41, 61, 62], with a fair amount of evidence now suggesting that, for spin cast films, before the blend laterally phase separates into a laterally segregated film (which is usually

\footnotetext{
${ }^{19}$ Vertical Phase Separation: phase separation into vertically layered phases, e.g. a bilayer, usually caused by preferential surface attraction.

${ }^{20}$ Lateral Phase Separation: phase separation into laterally coexisting phases, whether from an approximately homogeneous film or a bilayer film, resulting in a laterally segregated film.

${ }^{21}$ Vertically Segregated (film): layered phases with interfaces parallel to the surfaces.

${ }^{22}$ Laterally Segregated (film): 'column' phases with interfaces perpendicular to the surfaces.

${ }^{23}$ Lateral Phase Separation via a Transient Wetting Layer: vertical phase separation initially proceeds, due to preferential surface attraction, resulting in a vertically segregated film. This state is unstable, and lateral phase separation occurs, resulting in a laterally segregated film.
} 
(a)

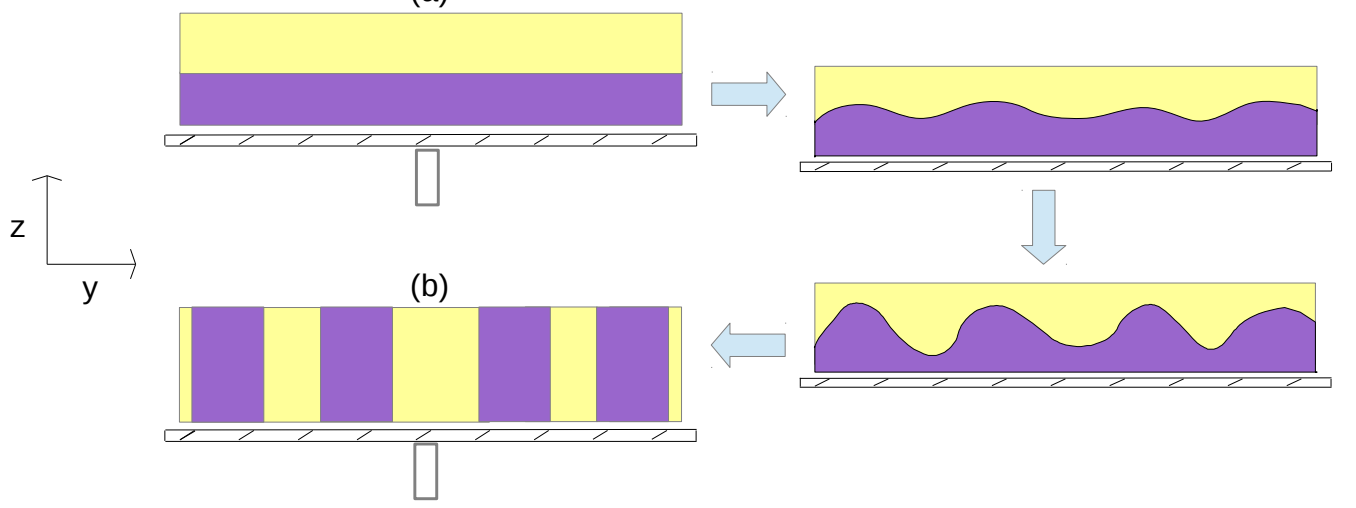

Figure 4.1: Schematics of different profiles for spin-cast films: (a) vertically segregated (bilayer); (b) laterally segregated. The process of spin-coating is shown in figure 4.2. There is evidence that films can in fact first undergo vertical phase separation into a bilayer, followed by a sinusoidal-like distortion of the interface between the phases of the bilayer, which results in lateral phase separation of the film (note that in (b) the coexisting phases are shown in common with chapters 2 and 3, with no consideration of the interface between them). In this case, the temporary bilayer is referred to as a transient wetting layer, and the process is referred to as lateral phase separation via a transient wetting layer. A posited reason for the breakup is a Marangoni-like instability; see text.

the final state of spin cast films) it first undergoes vertical phase separation into a bilayer (i.e. a transient wetting layer); see below for description of spin coating.

The most posited reason for the breakup of the bilayer is a Marangoni-like instability caused by solvent gradients in the film as solvent evaporates from the top surface $[60,62]$. This may be what causes the bilayer to develop a sinusoidal distortion which leads to the breakup of the bilayer, resulting in a laterally segregated film. Control of the evaporation rate, and therefore the steepness of the solvent gradient in the film, has been shown to be a mechanism by which the lateral phase separation can be prevented, allowing the final morphology to be either vertically or laterally segregated [62]. A schematic showing the process of the breakup of a bilayer is shown in figure 4.1.

Despite the recognition of lateral phase separation via a transient wetting layer, prior to this work little theoretical work had been done to shed light on the dynamics of the break up of the transient wetting layer, and any underlying mechanisms explaining the way in which the transient wetting layer breaks up.

\section{Spin Coating of Polymer Blend Films}

Spin coating is a widely used technique for creating polymer-blend thin films. A polymer blend is combined with a common solvent ('common' means that it dissolves both components of the blend), and sprayed/spread onto a flat substrate which is then spun very quickly. The fluid layer first thins from the spinning as material is rapidly shed due to centrifugal forces, and then thins further as solvent 


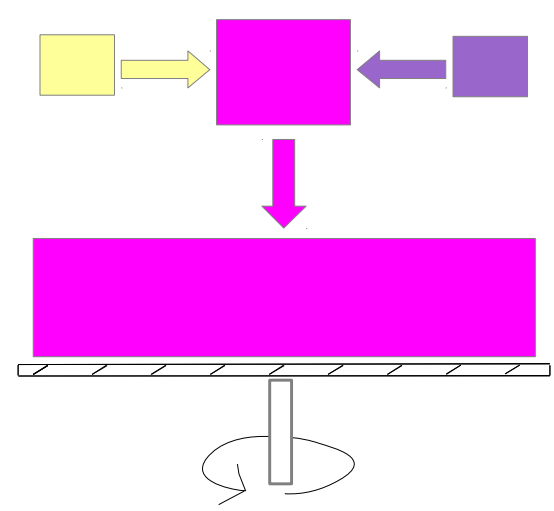

Figure 4.2: Schematic of initial stages of the spin-coating process: two polymer species are combined, along with a common solvent, and sprayed/spread onto a substrate, which is then spun around very quickly. The fluid layer thins as material is shed due to centrifugal forces, and thins further as solvent evaporates, leaving behind a film of the phase-separated polymer blend at the end.

evaporates, leaving behind a film of the binary polymer blend. The rate of solvent evaporation can be controlled by adjusting the vapour pressure above the film. See figure 4.2 for a simple schematic of the spin-coating process. The final state of films manufactured in this way is almost always laterally segregated, as in figure 4.1(b), but there is evidence that films first undergo vertical phase separation into a bilayer, as in Figure 4.1(a).

\subsection{Calculating phase equilibria in 1D}

The problem of solving for equilibrium profiles of polymer-blend thin films with selectively attracting surfaces (walls) was addressed in chapter 3 , but for completeness it is worth restating several important aspects in this chapter.

\subsubsection{D description of polymer-blend film}

The following description is consistent with chapter 3. The Flory-Huggins-de Gennes free energy functional $\mathcal{F}$ for a 1D binary polymer blend (monodisperse, components $\mathrm{A}$ and $\mathrm{B}$, volume fraction of A given by $\phi$, depth $d$ ) confined between selectively attracting walls (surfaces) at $z=0$ and $z=d$ is [18]

$$
\mathcal{F}[\phi(z)]=\frac{1}{a} \int_{0}^{d}\left[f_{F H}(\phi)+\kappa(\phi)(\nabla \phi)^{2}\right] d z+f_{0}^{*}\left(\phi_{0}\right)+f_{d}^{*}\left(\phi_{d}\right),
$$

where $\mathcal{F}$ is given in units of $k_{B} T, z$ measures the vertical distance from the wall at $z=0$, and $\nabla \phi \equiv \partial_{z} \phi$ is the partial derivative of $\phi$ with respect to $z$. I will denote film profiles, describing the volume fraction $\phi$ as a function of distance $z$, by $\phi(z) . a$ is the spacing of the underlying Flory-Huggins lattice. The gradient coefficient $\kappa(\phi)$ in Eq. (4.1) is

$$
\kappa(\phi)=\frac{a^{2}}{36 \phi(1-\phi)} .
$$


The surface energies $f_{0}^{*}$ and $f_{d}^{*}$ are given by $[31,33]$

$$
f_{S}^{*}\left(\phi_{S}\right)=h_{S}^{*} \phi_{S}+\frac{1}{2} g_{S}^{*} \phi_{S}^{2}=\frac{1}{a}\left(h_{S} \phi_{S}+\frac{1}{2} g_{S} \phi_{S}^{2}\right) \equiv \frac{1}{a} f_{S}\left(\phi_{S}\right),
$$

where $S=0, d$ (the index $S$ denotes the confining walls) and $h_{S}^{*} \equiv h_{S} / a$ and $g_{S}^{*} \equiv$ $g_{S} / a$ are phenomenological parameters, taking account of blend-wall interactions and missing neighbour contributions due to the walls (surfaces), respectively. Only the local volume fraction $\phi_{S}$ enters into Eq. (4.3). Note that the definitions $f_{S}\left(\phi_{S}\right)=h_{S} \phi_{S}+\frac{1}{2} g_{S} \phi_{S}^{2}$ are the surface energies per unit cell. These are convenient because $h_{S}$ and $g_{S}$ are independent of the dimensionality $\mathcal{D}$ of the system (which enters as $a^{-\mathcal{D}}$ in Eqs. (4.1) and (4.3), $a^{-1}$ in those cases).

To isolate the symmetry-breaking effects of the film walls, I now specialise to a symmetric binary polymer blend (average composition $\bar{\phi}=1 / 2$, degree of polymerisation $N_{A}=N_{B}=N$ ), so the Flory-Huggins free energy contribution to Eq. (4.1) in units of $k_{B} T$ is

$$
f_{F H}(\phi)=\frac{\phi}{N} \ln (\phi)+\frac{1-\phi}{N} \ln (1-\phi)+\chi \phi(1-\phi),
$$

where $\chi$ is the Flory-Huggins interaction parameter.

\subsubsection{The Chemical Potential}

In chapter 3, it was briefly noted that the Lagrange multiplier required to find equilibria with the Hamiltonian Phase Portrait Method was equivalent to a chemical potential. To recap: for any chosen blend ratio A:B (quantified by the average composition $\bar{\phi}$ ) equilibria implied to exist in isolation (rather than coexisting with other phases) must conserve $\bar{\phi}$, which for a symmetric binary blend means $\bar{\phi}=1 / 2$. The Lagrange multiplier must be chosen to ensure that calculated profiles satisfy the latter constraint. In a laterally segregated film of coexisting phases $\phi_{A}(z)$ and $\phi_{B}(z)$ (rich in components $\mathrm{A}$ or B i.e. $\bar{\phi}_{B}<\bar{\phi}<\bar{\phi}_{A}$ ), adjustment of the area and composition of each phase can conserve material, though $\lambda_{A}=\lambda_{B}$ is required $\left(\lambda_{A}=\lambda_{B}=0\right.$ was used in chapter 3$)$.

In $1 \mathrm{D}$, the total free energy is given by

$$
\mathcal{F}_{T O T}[\phi(z)]=\mathcal{F}[\phi(z)]-\frac{\mu}{a} \int_{0}^{d} \phi(z) d z,
$$

where a factor of $k_{B} T$ has been absorbed into the chemical potential $\mu$, which is multiplied by $a^{-1}$ in this definition for convenience. The local chemical potential $\mu(z)$ is related to the free energy via $\mu(z) \equiv a \delta \mathcal{F} / \delta \phi(z)$ (using a consistent definition as before). Since equilibrium profiles (equilibria) $\phi(z)$ correspond to a minimum in the total free energy i.e. $\delta \mathcal{F}_{T O T} / \delta \phi(z)=0$, then for equilibrium

$$
\frac{\delta \mathcal{F}[\phi(z)]}{\delta \phi(z)} \equiv \frac{\mu(z)}{a}=\frac{\mu}{a}
$$

meaning the local chemical potential $\mu(z)$ is a constant value $\mu$ for all $z$ at equilibrium. This was mentioned briefly in chapter 3 section 3.3.4 on the interpretation of the Lagrange multiplier $\lambda$. Another way to put this is to say: for equilibrium, there are no gradients in the chemical potential. 


\subsubsection{Solving for $1 \mathrm{D}$ equilibria}

I will briefly restate several equations relevant to the Hamiltonian Phase Portrait method, which equilibrium profiles are required to satisfy. These equations are discussed in detail in chapter 3. Underlying the Hamiltonian phase portrait method is the Euler-Lagrange equation for equilibrium profiles $\phi(z)$, given by

$$
2 \kappa \nabla^{2} \phi+\partial_{\phi} \kappa(\nabla \phi)^{2}=\partial_{\phi} f_{F H}+\lambda
$$

where $\partial_{\phi}$ is the partial derivative with respect to $\phi$, and $\lambda$ is a Lagrange multiplier. Eq. (4.7) is not enough to fully specify a solution; we require two boundary conditions to numerically solve for a unique solution:

$$
\begin{aligned}
& +2 \kappa\left(\phi_{0}\right) \nabla \phi_{0}=+\frac{\partial f_{0}}{\partial \phi} \equiv+h_{0}+g_{0} \phi_{0} \\
& +2 \kappa\left(\phi_{d}\right) \nabla \phi_{d}=-\frac{\partial f_{d}}{\partial \phi} \equiv-h_{d}-g_{d} \phi_{d} .
\end{aligned}
$$

We also need to satisfy a material constraint for independently-existing profiles, which for a symmetric blend $\bar{\phi}=1 / 2$ is

$$
\frac{1}{a} \int_{0}^{d}\left(\phi(z)-\frac{1}{2}\right) d z=0
$$

The boundary conditions (4.8) and (4.9) must be satisfied, along with the constraint equation (4.10) via choice of $\lambda$, to specify equilibrium profiles $\phi(z)$ which also satisfy the Euler-Lagrange equation $(4.7)$ [27, 31, 33]. Of course, that equilibria must satisfy these requirements is not specific to the Hamiltonian Phase Portrait method in particular.

\subsection{Modelling Phase Separation}

In order to study phase separating films, I use diffusion simulations based upon a diffusion equation in which the gradient of the local chemical potential drives the flux of material. I use 'simulation' throughout to mean numerically solving the resulting equation of motion. It is now useful to write Eq. (4.1) slightly differently, as

$$
a \mathcal{F}[\phi(z)]=\int_{0}^{d}\left[f_{F H}(\phi)+\kappa(\phi)(\nabla \phi)^{2}+f_{0}(\phi) \delta_{z 0}+f_{d}(\phi) \delta_{z d}\right] d z
$$

where $\delta_{z S}$ represents the Kronecker delta function: $\delta_{z S}(z=S)=1, \delta_{z S}(z \neq S)=$ $0(S$ refers to the value of $z$ at the walls i.e. $S=0$ or $S=d$ ). Note that since the film surfaces are rigid planar impenetrable surfaces, I will most often refer to the surfaces as walls. 


\subsubsection{The local chemical potential}

To derive the diffusion equation, we require the local chemical potential $\mu(z)$ as defined by $\mu(z) \equiv a \delta \mathcal{F}[\phi(z)] / \delta \phi(z)$. There is a standard expression for the functional derivative which can be used in cases where the boundaries of integration on a functional, such as $\mathcal{F}[\phi(z)]$ in equation (4.1), don't introduce additional terms into the functional derivative. In that case, for a functional of the form $F=\int f(\phi, \nabla \phi) d z$, we could use the expression

$$
\frac{\delta F[\phi(z)]}{\delta \phi(z)}=\frac{\partial f}{\partial \phi}-\nabla \cdot \frac{\partial f}{\partial \nabla \phi}
$$

I began the research that forms this chapter by using such a derivation. However, in order to unite the results of the simulations with the results I could obtain using the Hamiltonian Phase Portrait method to calculate equilibrium profiles, the diffusion equations for the simulations required modification. I found that terms resulting from one-sided gradients at the boundaries of integration, required for the equilibrium profiles from the simulations to match those calculated, were missing if I used equation 4.12. To try to find the source of the discrepancy, I instead went back to first principles to calculate the functional derivative.

The local chemical potential $\mu\left(z^{\prime}\right)$, given by the functional derivative of the free energy (4.1) at $z^{\prime}$, is:

$$
\mu\left(z^{\prime}\right)=a \frac{\delta \mathcal{F}[\phi(z)]}{\delta \phi\left(z^{\prime}\right)}
$$

The variational derivative in Eq. (4.13) describes how $\mathcal{F}$ changes when we perturb the profile $\phi(z)$ by an infinitesimally small amount $\epsilon$ at the point $z^{\prime}$ :

$$
\frac{\delta \mathcal{F}[\phi(z)]}{\delta \phi\left(z^{\prime}\right)}=\lim _{\epsilon \rightarrow 0} \frac{1}{\epsilon}\left\{\mathcal{F}\left[\phi(z)+\epsilon g\left(z, z^{\prime}\right)\right]-\mathcal{F}[\phi(z)]\right\}
$$

$g\left(z, z^{\prime}\right)$ is a test function (not rigorously a delta function or delta distribution) whose value is zero for $z \neq z^{\prime}$ and one for $z=z^{\prime}$. It is necessary to use such a test function due to the finite range of the integral in the functional Eq. (4.11).

I will begin with the variational derivative of the gradient term $\kappa(\phi)(\nabla \phi)^{2}$ in Eq. (4.11). I use the contractions $g \equiv g\left(z, z^{\prime}\right)$ and $\kappa \equiv \kappa(\phi)$ when convenient, and discard all terms $O\left(\epsilon^{2}\right)$ in the expanded integrands since they vanish in the limit $\epsilon \rightarrow 0$. Note that the quantity we are actually interested in is the local chemical potential $\mu(z)$ i.e. we must take $z=z^{\prime}$, so the integrals below vanish 
when we take $z^{\prime}=z$ due to the properties of the test function $g\left(z, z^{\prime}\right)$ :

$$
\begin{aligned}
\frac{1}{\epsilon} \int_{0}^{d} \kappa(\phi+\epsilon g)(\nabla(\phi+\epsilon g))^{2}-\kappa(\phi)(\nabla \phi)^{2} d z \\
=\int_{0}^{d} 2 \kappa \nabla \phi \nabla g+g\left(\partial_{\phi} \kappa\right)(\nabla \phi)^{2} d z \\
=\quad[2 \kappa \nabla \phi g]_{0}^{d}-\int_{0}^{d} 2 \kappa g \nabla^{2} \phi d z \\
\quad-\int_{0}^{d} 2 \nabla \phi g \nabla \kappa d z+\int_{0}^{d} g\left(\partial_{\phi} \kappa\right)(\nabla \phi)^{2} d z \\
=[2 \kappa \nabla \phi]_{0}^{d}-2 \kappa \nabla^{2} \phi-2 \nabla \phi \nabla \kappa+\left(\partial_{\phi} \kappa\right)(\nabla \phi)^{2} \\
=[2 \kappa \nabla \phi]_{0}^{d}-2 \kappa \nabla^{2} \phi-\left(\partial_{\phi} \kappa\right)(\nabla \phi)^{2} .
\end{aligned}
$$

The terms $[2 \kappa \nabla \phi]_{0}^{d}$ do not appear from equation (4.12), and these terms are usually missing from similar work in the literature.

The variational derivative of the Flory-Huggins free energy (4.4) is simply given by $\partial_{\phi} f_{F H}$, the partial derivative with respect to $\phi$

$$
\frac{\partial f_{F H}}{\partial \phi}=\frac{1}{N} \ln \frac{\phi}{1-\phi}+(1-2 \phi) \chi
$$

and the variational derivatives of the surface energies in Eq. (4.3) are $\left[\partial_{\phi} f_{S}(\phi)\right] \delta_{z S}$, due to the rewriting of the surface energies in the form given by equation (4.11), where (for $S=0, d$ )

$$
\frac{\partial f_{S}}{\partial \phi}=h_{S}+g_{S} \phi
$$

So we have for the local chemical potential $\mu(z)$ at point $z$ :

$$
\begin{aligned}
\mu(z)= & -2 \kappa(\phi) \nabla^{2} \phi-\left(\partial_{\phi} \kappa\right)(\nabla \phi)^{2}+\partial_{\phi} f_{F H} \\
& +\left[+2 \kappa(\phi) \nabla \phi+\partial_{\phi} f_{d}(\phi)\right] \delta_{z d} \\
& +\left[-2 \kappa(\phi) \nabla \phi+\partial_{\phi} f_{0}(\phi)\right] \delta_{z 0},
\end{aligned}
$$

where the surface energy terms and $[2 \kappa(\phi) \nabla \phi]_{0}^{d}$ have been combined, since both pairs of terms act at the walls.

\section{Consistency}

Eq. (4.6) means the chemical potential must be constant everywhere at equilibrium, $\mu(z)=\mu$. The Euler-Lagrange equation (4.7) must also be satisfied by equilibrium profiles $\phi(z)$. Substituting Euler-Lagrange equation (4.7) into the local chemical potential (4.18) we obtain for equilibrium

$$
\begin{aligned}
\mu(z) & =-\lambda \\
& +\left[+2 \kappa(\phi) \nabla \phi+\partial_{\phi} f_{d}(\phi)\right] \delta_{z d} \\
& +\left[-2 \kappa(\phi) \nabla \phi+\partial_{\phi} f_{0}(\phi)\right] \delta_{z 0},
\end{aligned}
$$


which requires that the boundary conditions Eqs. (4.8) and (4.9) are naturally satisfied, indeed the requirement that $\mu(z)$ be constant at equilibrium is the origin of the boundary conditions for equilibrium profiles. If we were to perform simulations of a polymer-blend described by equation (4.11), then we would expect to find for equilibrium profiles that

$$
\mu \equiv \mu_{\text {sim }}=-\lambda,
$$

i.e. at equilibrium, the Lagrange multiplier is the negative of the chemical potential $\mu_{\text {sim }}$ from the simulations. The relationship between $\lambda$, required to solve for profiles in $1 \mathrm{D}$, and the chemical potential from a $2 \mathrm{D}$ simulation in which there are lateral interfaces between coexisting phases (not accounted for when solving for equilibria in 1D) is discussed in section 4.4.

\subsubsection{The diffusion equation}

From the local chemical potential we can develop a governing equation for the diffusion simulations. I assume the material current at a point $z$ can be written as $J(z)=-M^{*} \nabla \delta \mathcal{F} / \delta \phi$, where $M^{*}$ is the mobility, assumed to be constant for simplicity. This constitutive law (a form of Fick's law of diffusion) assumes that the flux of material $J$ is proportional to the gradient of the chemical potential, meaning that material will move so as to equalise the chemical potential. This means that material will diffuse until equilibrium is achieved, in which the chemical potential is constant everywhere. From the continuity equation $\partial \phi / \partial t=-\nabla \cdot J$ we then obtain (remembering the factor of $a$ in the definition of $\mu$ )

$$
\frac{\partial \phi(z)}{\partial t}=\frac{M^{*}}{a} \nabla^{2} \mu(z) \equiv M \nabla^{2} \mu(z) .
$$

Inserting the expression for the local chemical potential (4.18) into the above expression yields the diffusion equation for the simulations. For numerical purposes, we scale space by $z^{\prime}=\left|\chi-\chi_{S}\right|^{1 / 2} z / a$ and time by $\tau=N M\left|\chi-\chi_{S}\right|^{2} t / a^{2} \cdot \chi_{S}$ is the value of $\chi$ at the spinodal, which for a symmetric blend gives $\chi_{S}=\chi_{C}$, where $\chi_{C}$ is the critical temperature of the blend (see section 1.4). The rescaled units of space $z^{\prime}$ are of the magnitude of phase separated domains expected from bulk phase separation, and the rescaled time $\tau$ removes the dependence on the mobility. We then obtain

$$
\begin{aligned}
& \frac{\partial \phi(z)}{\partial \tau}=\frac{1}{N} \nabla^{\prime 2}\left(\frac{1}{\left|\chi-\chi_{S}\right|} \frac{\partial f_{F H}}{\partial \phi}\right. \\
& +\frac{(1-2 \phi)}{\phi(1-\phi)} \frac{\kappa}{a^{2}}\left(\nabla^{\prime} \phi\right)^{2}-2 \frac{\kappa}{a^{2}} \nabla^{\prime 2} \phi \\
& +\frac{\delta_{z d}}{\left|\chi-\chi_{S}\right|}\left[\frac{\partial f_{d}}{\partial \phi_{d}}+2 \frac{\left|\chi-\chi_{S}\right|^{1 / 2}}{a} \kappa \nabla^{\prime} \phi\right] \\
& \left.+\frac{\delta_{z 0}}{\left|\chi-\chi_{S}\right|}\left[\frac{\partial f_{0}}{\partial \phi_{0}}-2 \frac{\left|\chi-\chi_{S}\right|^{1 / 2}}{a} \kappa \nabla^{\prime} \phi\right]\right) .
\end{aligned}
$$


where $\partial_{\phi} f_{F H}=(1 / N) \ln (\phi /(1-\phi))+\chi(1-2 \phi)$.

Eq. (4.22) must be discretised for simulations. I divide the range in $z$ by a mesh of $D$ grid cells of depth $\Delta z$, so the film depth $d=D \Delta z$ and the surface terms act in the grid cells $i=1$ and $i=D$ respectively. As first discussed by Henderson and Clarke [63], and later given firmer foundations by Fukuda et al [64], inconsistencies can arise unless we normalise surface/wall energy (4.3) to make the free energy (4.11) invariant to the mesh size:

$$
f_{i}\left(\phi_{i}\right) \rightarrow \frac{f_{i}\left(\phi_{i}\right)}{\Delta z}=\frac{h_{i}}{\Delta z} \phi_{i}+\frac{1}{2} \frac{g_{i}}{\Delta z} \phi_{i}^{2}
$$

where $i=1$ or $i=D$. The surface gradient terms $\pm 2 \kappa(\phi) \nabla \phi$ must also be normalised by $\Delta z^{-1}$, else the resulting discretised diffusion equation is not consistent with its continuous counterpart Eq. (4.22).

I also include a second lateral dimension $y$ running parallel to the confining walls, using index $j$, and apply periodic boundary conditions in this dimension. The free energy functional (4.1) changes such that $a^{-1} \rightarrow a^{-2}$, but the careful definitions means that we need only replace factors of $a^{-1}$ with $a^{-2}$ in Sections 4.2 and 4.3 (this leaves Eq. (4.20) remains unchanged). I used a square simulation mesh $\Delta y=\Delta z$ for the work in this chapter. Using $\phi_{i j}$ to represent the volume fraction of $A$ at the grid cell $i j$, the $2 \mathrm{D}$ discrete diffusion equation for the simulations discussed in this chapter is

$$
\begin{aligned}
& \frac{\partial \phi_{i j}}{\partial \tau}=\frac{1}{N} \nabla^{\prime 2}\left(\left.\frac{1}{\left|\chi-\chi_{S}\right|} \frac{\partial f_{F H}}{\partial \phi}\right|_{i j}\right. \\
& +\frac{\left(1-2 \phi_{i j}\right)}{\phi_{i j}\left(1-\phi_{i j}\right)} \frac{\kappa_{i j}}{a^{2}}\left(\left.\nabla^{\prime} \phi\right|_{i j}\right)^{2}-\left.2 \frac{\kappa_{i j}}{a^{2}} \nabla^{\prime 2} \phi\right|_{i j} \\
& +\frac{\delta_{i D}}{\Delta z^{\prime}}\left[\frac{a^{-1}}{\left|\chi-\chi_{S}\right|^{\frac{1}{2}}} \frac{\partial f_{d}}{\partial \phi_{d}}+\left.2 \frac{\kappa_{D j}}{a^{2}} \nabla_{z}^{\prime} \phi\right|_{D j}\right] \\
& \left.+\frac{\delta_{i 1}}{\Delta z^{\prime}}\left[\frac{a^{-1}}{\left|\chi-\chi_{S}\right|^{\frac{1}{2}}} \frac{\partial f_{0}}{\partial \phi_{0}}-\left.2 \frac{\kappa_{1 j}}{a^{2}} \nabla_{z}^{\prime} \phi\right|_{1 j}\right]\right),
\end{aligned}
$$

where $\kappa_{i j} \equiv \kappa\left(\phi_{i j}\right)$ and $\left.\partial_{\phi} f_{F H}\right|_{i j} \equiv \partial_{\phi} f_{F H}\left(\phi_{i j}\right)$. The gradient terms $\nabla^{\prime}$ and $\nabla^{\prime 2}$ are now $2 \mathrm{D}$, whilst $\nabla_{z}^{\prime} \equiv \partial_{z^{\prime}}$ (the partial derivative with respect to $z^{\prime}$ ). For the surface terms $\partial f_{d} / \partial \phi_{d}$ and $\partial f_{0} / \partial \phi_{0}$, I keep the notation for the continuous spatial variable $z(0$ and $d)$, although these terms must be evaluated for grid cells with $i=1, D$. (Note that the lattice spacing $a$ cannot be fully scaled out of the surface terms; see ref [64]).

The film surfaces are impenetrable hence material conservation is required:

$$
\frac{d}{d \tau} \int_{0}^{d} \phi(z) d z=0
$$

which was implemented by a no-flux condition at the walls, achieved by setting $J(i=1-1 / 2)=0$ and $J(i=D+1 / 2)=0$. To discretise Eq. (4.24), I used a central differencing scheme for spacial derivatives and a forward differencing 
time step. Unless specified otherwise, simulations are started seeded with initial random noise i.e. $\phi(z, y)=0.5+\delta \phi$, where $\delta \phi$ is chosen from a Gaussian distribution with mean zero and width $\sigma$, which will be specified, and additional noise is not included throughout the rest of the simulation. However, to show that the results are general, I show a simulation with continuous noise in section 4.6.1.

The simulations were implemented in CUDA, a programming language similar to $\mathrm{C}++$ which allows for execution of parallelised code on a CUDA-enabled NVIDIA Graphical Processing Unit (GPU). This made the simulations much more tractable, providing between $10 \times$ to $100 \times$ speed up over serial processing on a CPU. More details of the implementation of my simulations for parallelised code, which may be useful for reproducing them or for efficiently implementing other similar diffusion simulations, along with details about the discretised gradient operators and time step, are given in appendix A.

\subsubsection{Parameters}

\section{Blend and surface parameters}

Throughout this chapter, I focus on a symmetric polymer blend $\bar{\phi}=0.5$ with $N=$ 100 , hence $\chi_{c}=0.020$. I use the following wall parameters and terminology, in common with chapter 3 : for 'asymmetric' films $h_{0}=-0.05, g_{0}=0.18, h_{d}=g_{d}=$ 0 (a $B$-attracting wall at $z=0$ and a neutral wall at $z=d$ ); for 'antisymmetric' films $h_{0}=-0.05, g_{0}=0.18, h_{d}=-0.13, g_{d}=0.18$ (a $B$-attracting wall at $z=0$ and an $A$-attracting wall at $z=d$, such that the walls attract opposite components in exactly the same way); and for 'symmetric' films $h_{0}=h_{d}=-0.05$, $g_{0}=g_{d}=0.18$ (a $B$-attracting wall at $z=0$ and $z=d$ ). These different surface regimes are discussed in chapter 2 section 2.5. The symmetric blend between asymmetric walls is directly comparable with the work in chapter 3 .

\section{Parameters and units}

Since scaled space depends on the temperature $\chi$ and I discuss a range of different $\chi$, it is easier to discuss the results in terms of 'unscaled' space $z$ (setting $a=1$ gives $z$ units of $a$ ) and scaled time $\tau$, since then the difference in behaviour for films of the same depth at different temperatures is more transparent (the scaling of space is nonetheless useful for numerical reasons). When discussing phase portraits, I provide the Hamiltonian constants and Lagrange multipliers used to generate the phase portraits.

\section{The wetting temperature}

Since the wetting temperature depends on the film thickness and wall interactions, I have defined the wetting temperature $\chi_{W}$ throughout this chapter as the cutoff temperature at which spontaneous lateral phase separation of a transient wetting layer no longer appears to occur (i.e. the wetting layer is stable, and the contact angle of the wetting phase with the surface remains zero) for an asymmetric film of depth $d=20.1$ (the film depth used in section 4.5). This 


\begin{tabular}{ccccc}
\hline \hline$\chi$ & $\lambda$ & $d$ & $\Delta z$ & $\mu_{\text {sim }}$ \\
\hline 0.015 & -0.000745 & 14.85 & 0.10 & +0.000745 \\
& & & 0.41 & +0.000791 \\
& & & 0.74 & +0.000832 \\
& & & 1.24 & +0.000897 \\
0.021 & -0.000033 & 40.60 & 0.25 & +0.000033 \\
& & & 0.68 & +0.000036 \\
& & & 1.13 & +0.000037 \\
& & & 1.69 & +0.000039 \\
\hline 0.026 & +0.000120 & 20.92 & 0.20 & -0.000120 \\
& & & 0.40 & -0.000118 \\
& & & 0.65 & -0.000116 \\
& & & 1.05 & -0.000112 \\
& & & 1.74 & -0.000101 \\
\hline
\end{tabular}

Table 4.3: Lagrange multiplier $\lambda$ (for the calculated 1D profile) and equilibrium chemical potential $\mu_{\text {sim }}$ (obtained from simulations) for different temperature regimes $\chi$, depths $d$, and varying mesh size $\Delta z$ (including all the data from the simulations for Figs. 4.3-4.5). As the mesh size becomes finer $\Delta z \rightarrow 0$, we observe $\mu \rightarrow-\lambda$, as predicted by Eq. (4.20). The rate equation (4.24) is therefore accurate and precise.

gives an estimate of $0.0213<\chi_{W}<0.0214$ (this provides an upper limit for all of my wall configurations, since my asymmetric configuration has one neutral wall; see ref [45]). It is worth restating that defining the wetting temperature as the point at which wetting is stable is really the fundamental definition of the wetting temperature. My more restricted definition here is for convenience, allowing us to refer to the same value of $\chi_{W}$ throughout, even though, for example, the 'true' wetting temperature of, say, an antisymmetric film may in fact be $\chi_{W}^{\mathrm{AS}}<\chi_{W}$.

\subsection{D equilibria in $2 \mathrm{D}$ films}

This section relates the phase equilibria that can be calculated in $1 \mathrm{D}$ via the Hamiltonian Phase Portrait Method of chapter 3, to the equilibrium states found in my 2D simulations. The purpose of this comparison was to investigate whether the simulations of the diffusion equation (4.24) were consistent with the equilibria calculated with the Hamiltonian phase portrait method, in particular whether the coexisting phases that can be calculated in $1 \mathrm{D}$ actually correspond to the laterally segregated phases observed in $2 \mathrm{D}$.

\subsubsection{Vertically segregated films}

To study vertically segregated films, such as figure 4.1(a), I restricted the simulations to quasi-1D, so that the lateral dimension $y$ was too narrow to support laterally segregated phases. For the Gaussian noise width I used $\sigma=0.0001$ for an initially nearly homogeneous film, and made a further restriction to asymmetric films. The equilibrium profiles from the quasi-1D simulations can be directly compared with calculated 1D film profiles of independently-existing equilibria, 


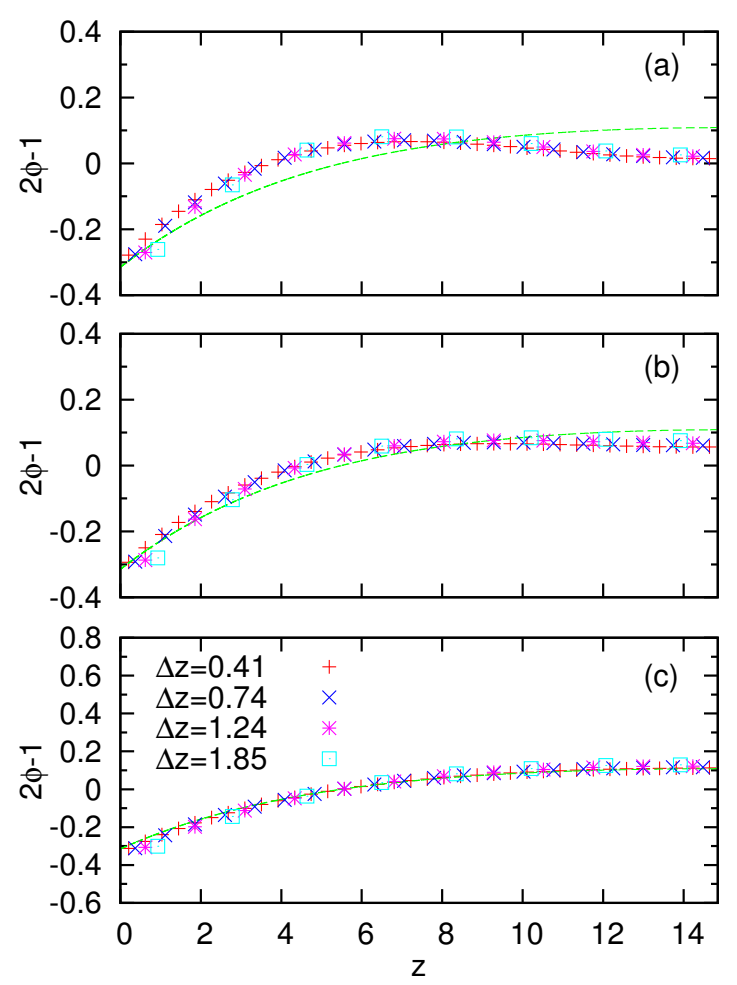

Figure 4.3: $1 \mathrm{D}$ simulation data for $\chi=0.015\left(\chi<\chi_{C}\right)$, film depth $d=14.85$ and asymmetric walls. Times are $\tau=1$ (a), $\tau=2$ (b), $\tau=15$ (c). The calculated equilibrium profile (green dashed line) required $\lambda=-0.000745$, whilst $\mu_{\text {sim }}=+0.000791$ for $\Delta z=0.41$. Equilibrium is a monolayer with positive adsorption of $\mathrm{B}$ material at the $\mathrm{B}$ attracting wall $(z=0)$.

since restricting the lateral width prevents lateral phase separation and therefore allows the vertically segregated state to be accurately compared with calculated profiles, even for $\chi>\chi_{W}$ when this configuration is unstable with respect to a laterally segregated configuration (any lateral variation in the vertically segregated profile would make a comparison less accurate). I studied three different temperature regimes (those used in chapter 3): above the critical temperature $\chi=0.015$ (figure 4.3), below the critical temperature but above the wetting temperature $\chi=0.021$ (figure 4.4), and below the wetting temperature $\chi=0.026$ (figure 4.5).

In Figs. 4.3, 4.4 and 4.5 the three sub-figures show each film at (a) a very early time; (b) an intermediate time away from equilibrium; and (c) at equilibrium. The data points $\phi_{i}(1 \leq i \leq D)$ are averages of $\phi_{i j}$ taken over index $j$ (the lateral dimension) for fixed index $i$, and the dashed lines are the 1D equilibrium profiles of asymmetric films calculated by the Hamiltonian phase portrait method of chapter 3, although any method would suffice. I made the following observations: at equilibrium the simulation data matches the profiles; the simulations reproduce the profiles more accurately as the mesh size is reduced $(\Delta \tau=0.00004$ was used for each temperature regime); and as $\Delta z \rightarrow 0$, the simulation data conforms exactly to the calculated profiles. Further proof of this is table 4.3, which shows that as $\Delta z \rightarrow 0$ the simulation equilibrium chemical potential converges to the 

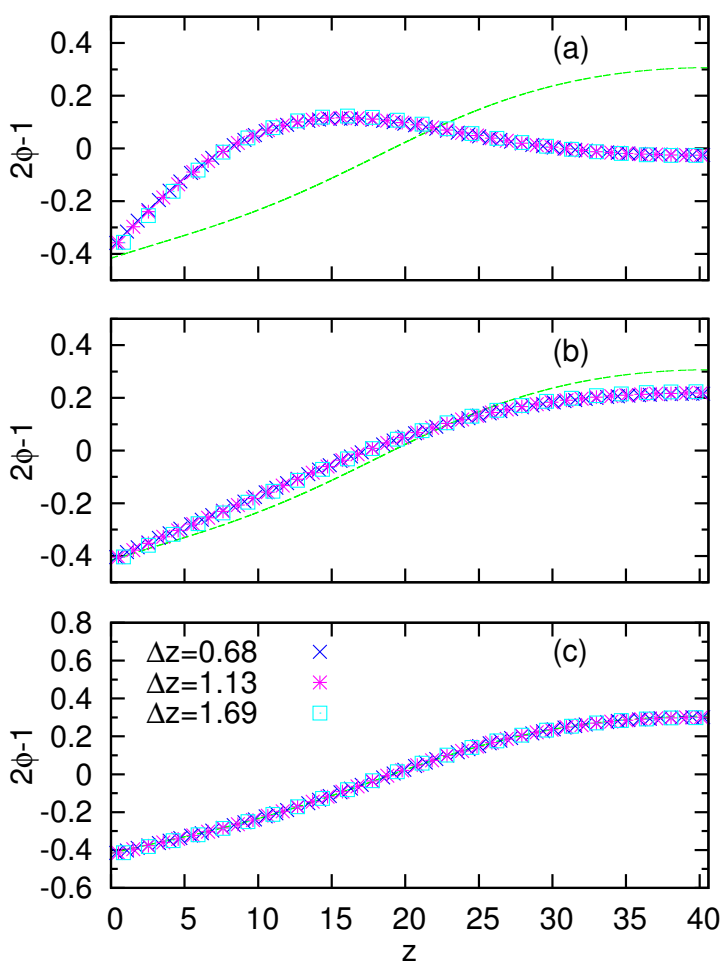

Figure 4.4: $1 \mathrm{D}$ simulation data for $\chi=0.021\left(\chi_{C}<\chi<\chi_{W}\right)$, film depth $d=40.60$ and asymmetric walls. Times are $\tau<1$ (a), $\tau=9$ (b), $\tau=25$ (c). The calculated equilibrium profile (green dashed line) required $\lambda=-0.000033$, whilst $\mu_{\text {sim }}=+0.000036$ for $\Delta z=0.68$. Equilibrium is a bilayer with a soft interface separating the B-rich phase and A-rich phase.

negative of the Lagrange multiplier required to numerically solve for the film profile, as predicted by Eq. (4.20), but these finer $\Delta z$ are absent in Figs. 4.3-4.5 to preserve clarity. It is important that the results of the simulation accurately and precisely match the results of a calculation of equilibria, since this gives us confidence in the diffusion equation (4.24).

It is worth noting that for thicker films an oscillatory concentration wave may form, in which the profile would resemble a sine-wave in concentration extending away from the $B$-attracting wall. This wouldn't necessarily become a profile like those in figures $4.3-4.5$ (unless given an infinite amount of time), but this is a kinetic problem that can be a particular nuisance in 1D simulations. Since the work in chapter 3 gives us confidence that we know the nature of the true equilibria even for infinitely thick films, and since the focus of this chapter is on lateral phase separation via a transient wetting layer, in which the transient wetting layer is a bilayer structure like that in figure 4.1(b), the problem of oscillatory profiles is not relevant.

\subsubsection{Pinning of profiles at the walls}

From Figs. $4.3-4.5$ we see, in agreement with the literature e.g. [57], that the value of $\phi$ at the B-attracting wall appears to be very quickly pinned to its 

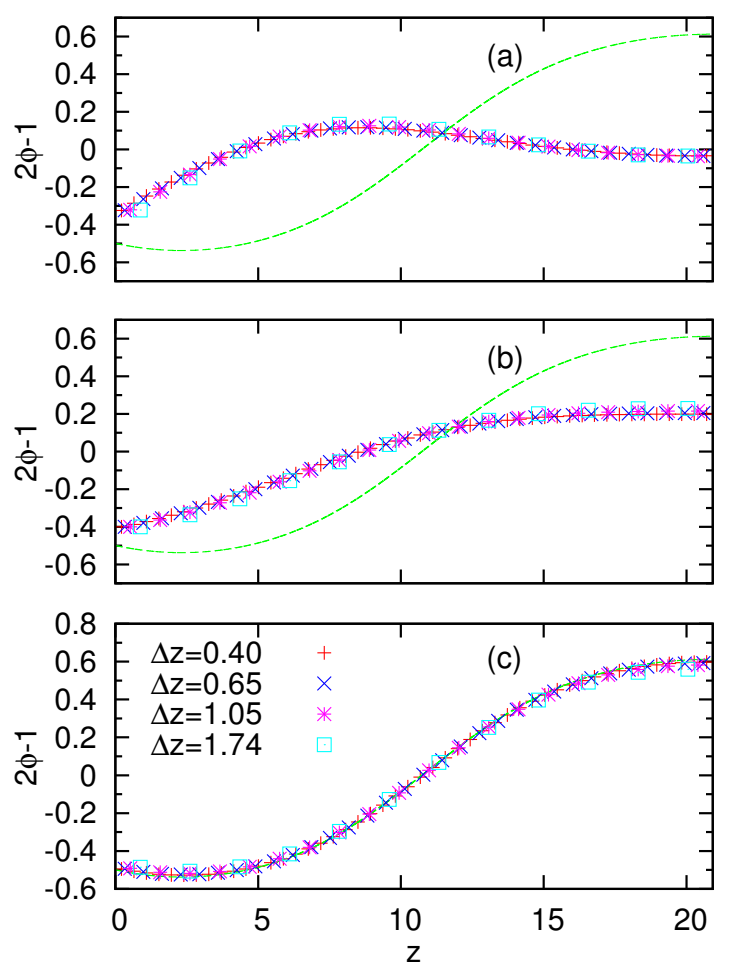

Figure 4.5: 1D simulation data for $\chi=0.026\left(\chi_{W}<\chi\right)$, film depth $d=20.92$ and asymmetric walls. Times are $\tau=2(\mathrm{a}), \tau=20$ (b), $\tau=100$ (c). The calculated equilibrium profile (green dashed line) required $\lambda=+0.000120$, whilst $\mu_{\text {sim }}=-0.000118$ for $\Delta z=0.40$. The equilibrium profile is non-monotonous with a minimum $(\nabla \phi=0)$ at $z \approx 2.5$.

equilibrium value, and from this pinning centre a concentration wave is crossing the film. On the other hand, the profile near the neutral wall is unperturbed at very early times, and it would appear that there is no such rapid pinning of $\phi$ at the neutral wall. For each temperature regime, a point is reached when the concentration wave has crossed the film, and the profiles show a monotonous increase in B towards the B attracting wall at $z=0$ and flat profile at $z=d$. For figure 4.5, the film then develops non-monotonous behaviour at $z \approx 2.5$ (characterised by a minimum in $\phi$ near but not at the B-attracting wall) shortly prior to achieving equilibrium (metastable in the latter case; $\chi>\chi_{W}$ ).

A more careful inspection of the diffusion equation (4.24) and boundary conditions (4.8) and (4.9) shows us that the profile is in fact pinned at both the B-attracting wall and the neutral wall, but that pinning ${ }^{24}$ does not refer to just the volume fraction $\phi$, but rather to both coordinates $\left(\phi, 2 \kappa \nabla_{z} \phi\right)$. The surface terms of the diffusion equation (4.24) effectively enforce the boundary conditions (4.8) and (4.9) such that they are fulfilled at all times during the simulation, not only at equilibrium. For the B-attracting wall, although $\phi$ is pinned at early times, it in fact does continue to change slightly, as does $\nabla_{z} \phi$. For the neutral wall, Eq. (4.9) with $h_{d}=g_{d}=0$ shows us that $\nabla_{z} \phi=0$ solves the bound-

\footnotetext{
${ }^{24}$ Pinning: values of $\left(\phi, 2 \kappa \nabla_{z} \phi\right)$ at the film surfaces are determined by surface boundary conditions, such that the ends of trajectories are always pinned to these boundary conditions.
} 


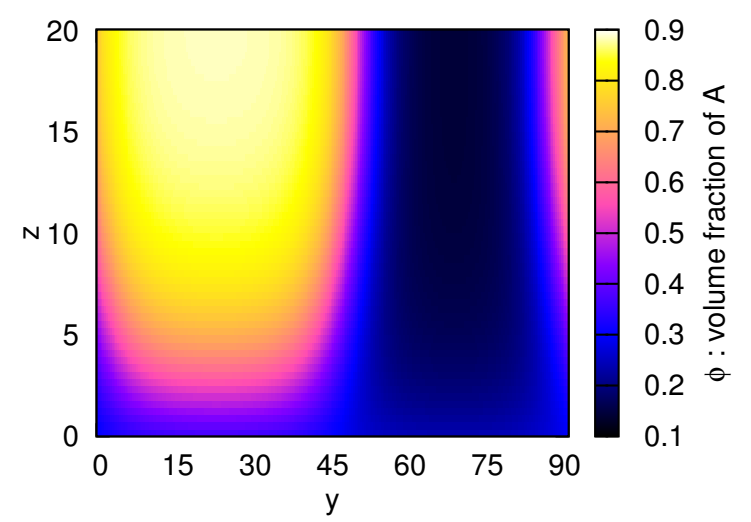

Figure 4.6: Laterally segregated phases for $\chi=0.026\left(\chi>\chi_{W}\right)$, depth $d=20.1$ and asymmetric walls, using $\Delta z=0.36$ and $\Delta \tau=0.10 \times 10^{-5}$. The lateral dimension $y \approx 90$ (with periodic boundary conditions) is wide enough to support two lateral phases.

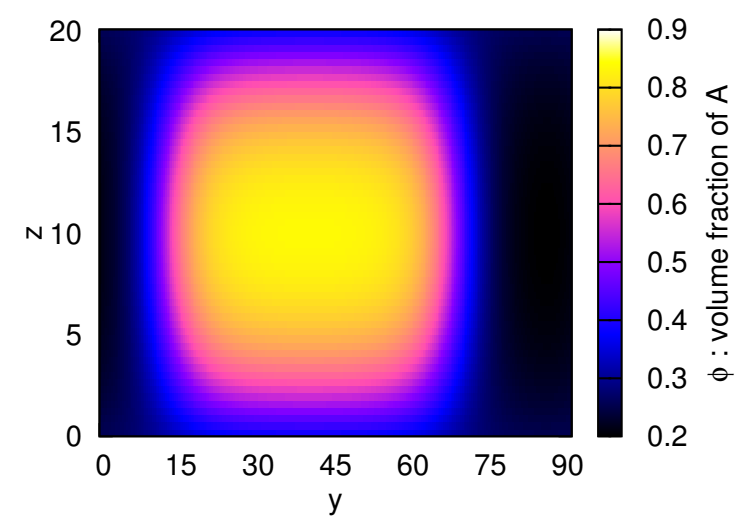

Figure 4.7: The laterally segregated state for $\chi=0.026\left(\chi>\chi_{W}\right)$, depth $d=20.1$ and symmetric walls, using $\Delta z=0.36$ and $\Delta \tau=0.25 \times 10^{-5}$. The lateral dimension $y \approx 90$ (with periodic boundary conditions) is wide enough to support two lateral phases.

ary condition for any value of $\phi$, so although the value of $\phi$ does not appear to be pinned, $\nabla_{z} \phi$ is pinned to zero. The wall-blend interactions thus enforce the boundary conditions at all times, but $\left(\phi, 2 \kappa \nabla_{z} \phi\right)_{0, d}$ may still change whilst satisfying these boundary conditions. This novel observation will be important to my discussion of lateral phase separation in section 4.5 , in which a graphical interpretation of this pinning can be made by considering phase portraits: no matter how the trajectories of the film profiles change during film evolution, the ends of the trajectories are pinned to the boundary conditions (4.8) and (4.9).

\subsubsection{Laterally segregated films}

I now discuss 2D simulations at $\chi>\chi_{W}$, for which global equilibrium is a laterally segregated film (to put it another way, if the film laterally segregates after temporary wetting, then $\chi>\chi_{W}$ by definition, since wetting is not stable). I will reserve discussion of the dynamics of lateral phase separation for section 4.5, and focus here on showing that the coexisting equilibria that can be calculated 


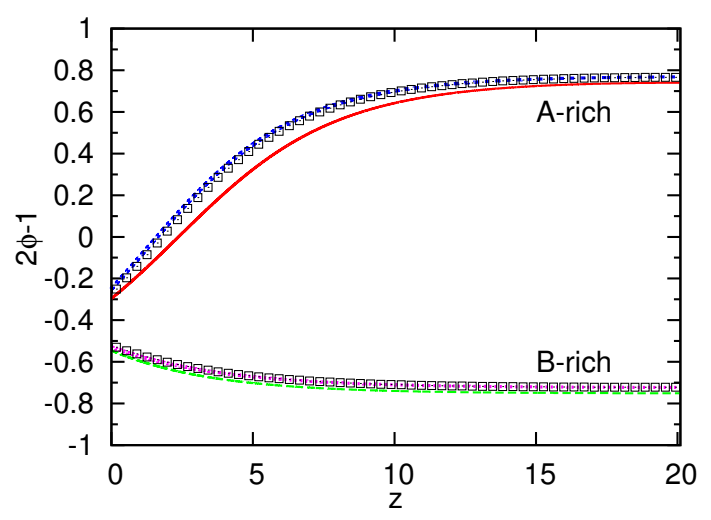

Figure 4.8: 1D vertical cross-sections from Fig. 4.6, (data points) and calculated profiles (curves) of coexisting phases for $\chi=0.026\left(\chi>\chi_{W}\right)$, depth $d=20.1$ and asymmetric walls. The curves matching the data points (blue and pink) were calculated with $\lambda=-\mu_{\text {sim }}=-0.000491\left(\mathcal{H}_{A}=0.001357, \mathcal{H}_{B}=0.000988\right)$, whilst the other curves (red and green) were calculated with $\lambda=0\left(\mathcal{H}_{A}=0.000923, \mathcal{H}_{B}=0.000924\right)$.

in 1D (in the dimension running between the walls) do in fact occur in laterally segregated 2D films.

\section{Comparison with 1D profiles}

Figs. 4.6 and 4.7 show, for an asymmetric and a symmetric film respectively, only the laterally segregated state corresponding to global equilibrium, which should technically consist of only a single pair of coexisting phases in contact. Note that the phases do not exist as side-by-side 'columns' as in previously shown schematics of laterally segregated films in this thesis, but form distinct shapes due to the interface between them. Although it's not clear where one would measure a contact angle (figure 2.2) with the surfaces in most cases, clearly the contact angle is generally not $90^{\circ}$. I previously argued in chapter 3 , on the subject of solving for these coexisting phases in $1 \mathrm{D}$, that since a Lagrange multiplier $\lambda \neq 0$ always acts to increase the free energy of a profile relative to the same profile for $\lambda=0$ ('same profile' in this context means that the solution trajectory through phase space is qualitatively the same, except for distortion due to non-zero $\lambda$; see section 4.5) that the coexisting solutions should be calculated in 1D for $\lambda=0$ [46]. Since actual coexistence of these lateral phases in a $2 \mathrm{D}$ film requires an interface we should expect $\lambda_{A}=\lambda_{B} \neq 0$.

To compare the 2D simulation data of figure 4.6 and 4.7 with the $1 \mathrm{D}$ coexisting phases calculated via a 1D Hamiltonian method (requiring a choice of $\lambda$ and Hamiltonian $\mathcal{H}$, explained in chapter 3 and section 4.5), I took 1D cross sections of the $2 \mathrm{D}$ data at points $y_{A}$ and $y_{B}$, which are at the cores/centres of the A-rich and B-rich phases respectively. The cross-sections from the asymmetric film of figure $4.6\left(y_{A} \approx 20\right.$ and $\left.y_{B} \approx 70\right)$ are shown in figure 4.8 , which also contains two pairs of curves. The curves to which no data points are directly aligned were obtained from a 1D calculation of the lateral phases using $\lambda=0$. However, the simulation for figure 4.8 gave $\mu_{\text {sim }}=0.000491$, so it is unsurprising that the data 


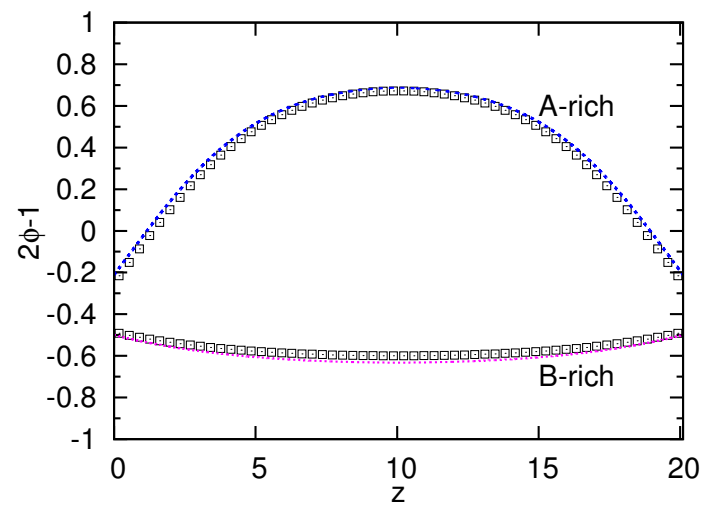

Figure 4.9: 1D vertical cross-sections from Fig. 4.7, (data points) and calculated profiles (curves) of coexisting phases for $\chi=0.026\left(\chi>\chi_{W}\right)$, depth $d=20.1$ and symmetric walls. The calculated profiles required $\lambda=-\mu_{\text {sim }}=-0.001202\left(\mathcal{H}_{A}=\right.$ $\left.0.001922, \mathcal{H}_{B}=0.001092\right)$. It is not possible to obtain A-rich profiles of depth $d=20.1$ for $\lambda=0$.

and the 1D calculated profiles do not coincide. The second set of curves, which are almost obscured by the data points, are the 1D profiles calculated with Lagrange multiplier $\lambda=-\mu_{\text {sim }}=-0.000491$. These $1 \mathrm{D}$ cross sections, whilst describing the majority of the cores of the phases very accurately, do not of course describe the interface between the phases. The cross-sectional profiles obtained from the data for the symmetric film of figure $4.7\left(y_{A} \approx 40\right.$ and $\left.y_{B} \approx 86\right)$ are shown in figure 4.9, along with $1 \mathrm{D}$ coexisting phases calculated for $\lambda=-\mu_{\text {sim }}=-0.001202$; a pair of A-rich and B-rich coexisting phases of depth $d=20.1$ cannot be calculated for $\lambda=0$. The relatively steep gradients in the A-rich phase cause some minor discretisation errors, causing the B-rich phase of the symmetric film to be slightly less B-rich than expected. No data is shown for antisymmetric films, since in this special case $\lambda=-\mu_{\text {sim }}=0$, and so we find that the lateral interfaces between the perfectly antisymmetric 1D phases do not introduce a non-zero chemical potential. I conclude that the laterally segregated state is effectively described by $1 \mathrm{D}$ equilibria in the dimension running perpendicular to the confining walls.

\section{Is there a minimum depth of solution?}

In chapter 3, I discussed how the minimum depth of A-rich solutions available with $\lambda=0$ for asymmetric films suggested a minimum depth of film required for lateral phase separation to occur, for a particular $\chi$. This idea is relevant here for symmetric films. Suitable adjustment of $\lambda$ could presumably produce coexisting states of any depth, but the relevant question is really: is the free energy of a 'thin' A-rich phase coexisting with a 'thin' B-rich phase (calculated by $\lambda \neq 0$ such that a 'thin' A-rich phase can actually be found) less than the free energy of an independently existing solution of the same depth? If not, then lateral phase separation is not preferable for that 'thin' film at that particular $\chi$. This is only a minor point with regards to the work in this chapter, since we are interested in the kinetics of phase separation, not in identifying the wetting temperature as a 


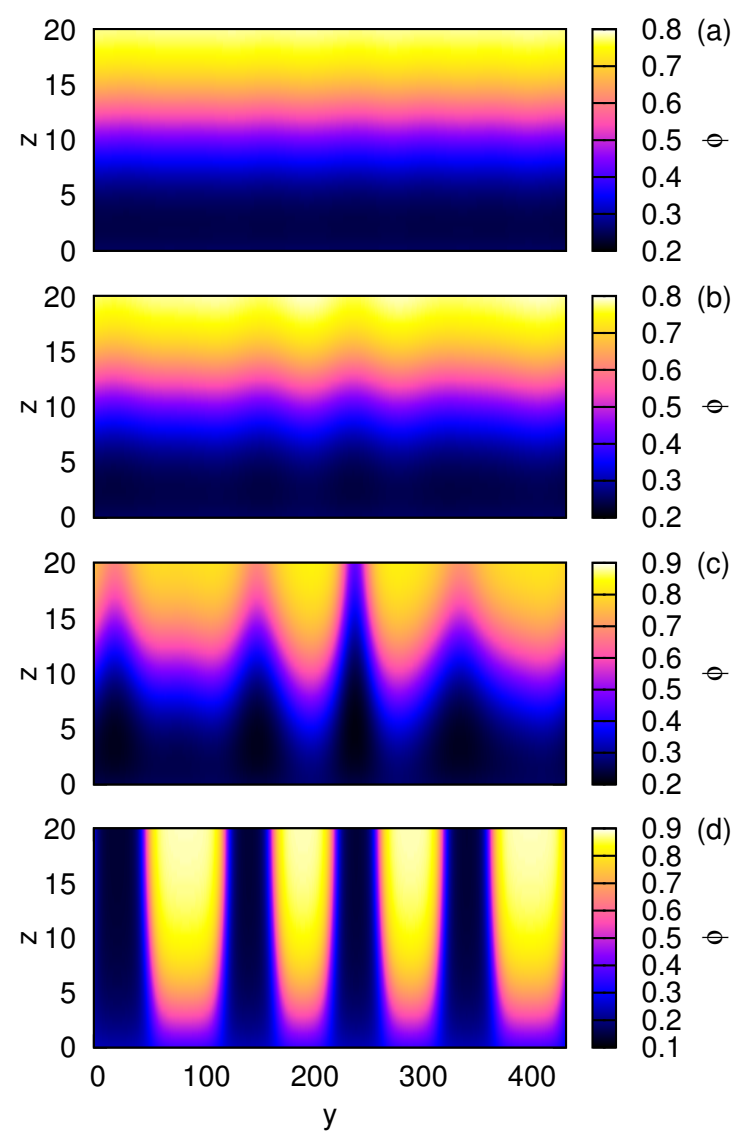

Figure 4.10: Simulation snapshots for $\chi=0.026\left(\chi>\chi_{W}\right), d=20.1$ and asymmetric walls $\left(\Delta z=0.50, \Delta \tau=1.0 \times 10^{-5}\right)$. (a) metastable bilayer state with minor lateral inhomogeneities $(\tau=100)$; (b) distortion of bilayer interface with corresponding inhomogeneities at the walls $(\tau=892)$ (c) break-up of the bilayer interface as a column of B-rich material reaches the $z=d$ wall $(\tau=1665)$; (d) laterally segregated coexisting states $(\tau=3655)$.

function of film depth $\chi(d)$. If at a particular value of $\chi$ lateral phase separation via a transient wetting layer occurs, then for this particular depth of film we know that $\chi(d)>\chi_{W}(d)$, and this is sufficient.

\subsection{Breakup of a Transient Wetting Layer}

In this section I discuss the dynamics of the breakup of a transient wetting layer, the dynamics by which the vertically segregated state is reached having been effectively discussed in the last section. Figs. 4.10, 4.12, 4.14, 4.16 and 4.18 show, for $\chi>\chi_{W}$, simulation snapshots of films undergoing lateral phase separation via a transient wetting later. The width of the initial noise $\delta \phi$ was $\sigma=0.05$, this choice allowing both the TWL (Transient Wetting Layer) and laterally segregated state to be probed.

It is important to highlight here that the absence of a random noise term in the current model means that the final lateral states I present do not always 


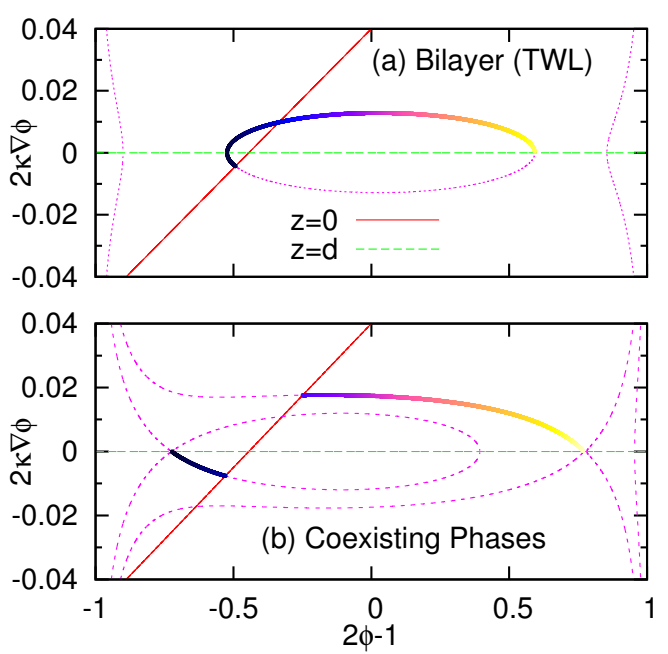

Figure 4.11: 1D phase portraits for equilibria in Fig. 4.10 (asymmetric walls). (a) Metastable bilayer (TWL) $(\lambda=+0.000119, \mathcal{H}=0.000743)$; (b) Coexisting phases $\left(\lambda=-\mu_{\text {sim }}=-0.000497\right)$ : A-rich phase $\left(\mathcal{H}_{A}=0.001362\right)$ and B-rich phase $\left(\mathcal{H}_{B}=\right.$ $0.000989) . \phi_{d}$ of all three phases are rather distinct. $\phi_{0}^{T W L}$ and $\phi_{0}^{B}$ are quite similar, but both distinct from $\phi_{0}^{A}$.

consist of 'wide' lateral phases (resulting from the merging of narrower lateral phases, for example) which are closer to 'true' global equilibrium (a single pair of laterally coexisting phases). However, the distinction between multiple lateral phases (wide or narrow) and a single pair of laterally coexisting phases is of little practical relevance to the mechanism I discuss here, or to most experiments. The lateral phases that appear in my simulations are the same phase equilibria that correspond to global equilibrium, and I refrain from using the latter term only because it could possibly be misleading (coarsening of the lateral phase in the simulations is technically possible). I present a simulation in which continuous random noise was included in section 4.6.1 figure 4.21, which shows that the results still hold in the case of continuous noise, and that wider lateral phases form in that case.

The wall configuration of the asymmetric film (a B-attracting wall and a neutral wall) is a special case in that there is only one surface field. This particular case highlights the qualitatively different behaviour at each confining surface and does not include any convenient symmetries that fix the chemical potential (e.g. $\mu(z)=0$ for antisymmetric films). General asymmetry complicates my discussion of the phase portrait method and would leave me short of discussing behaviour at a neutral wall. However, a neutral wall is nonetheless a special case, and so after discussing asymmetric films in section 4.5.1. I will extend my discussion to two non-zero surface fields in section 4.5.2.

\section{Phase portraits and profile pinning by boundary conditions}

In this section, I make use of Hamiltonian phase portraits, Figs. 4.11, 4.13, 4.15, 4.17 , and 4.19 , to discuss the $1 \mathrm{D}$ phase equilibria. These portraits are discussed in more detail in chapter 3 , but I will briefly recap them here. The Hamiltonian 


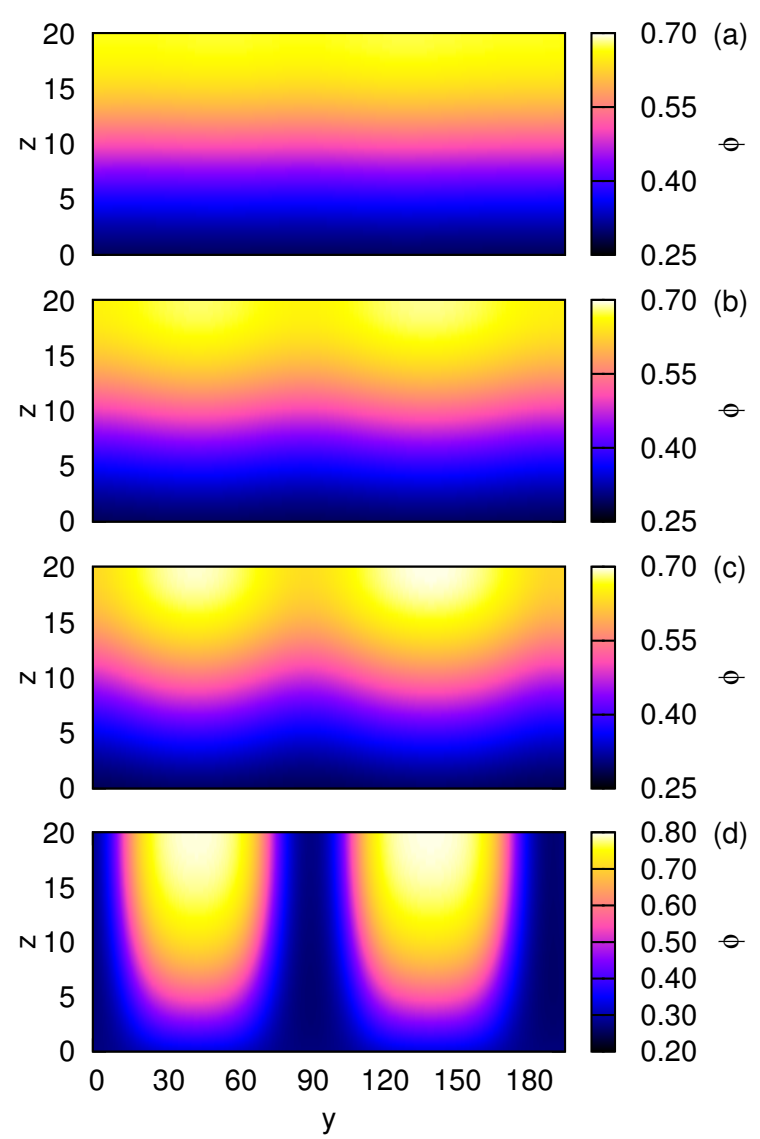

Figure 4.12: Simulation snapshots for $\chi=0.023\left(\chi>\chi_{W}\right), d=20.1$ and asymmetric walls $\left(\Delta z=0.50, \Delta \tau=0.5 \times 10^{-5}\right)$. (a) metastable bilayer state $(\tau=200)$; (b) distortion of bilayer interface as lateral structures grow primarily at $z=d$ wall $(\tau=$ $550)$; (c) A-rich phases growing from $z=d$ surface $(\tau=700)$ (d) laterally segregated coexisting states $(\tau=1500)$.

phase portraits consist of the flow of canonical coordinates $\left(\phi, 2 \kappa \nabla_{z} \phi\right)$ which minimise the bulk free energy functional $\mathcal{F}(4.11)$. Due to the coordinate space, the satisfied boundary conditions enforced by the walls (Eqs. (4.8)-(4.9)) are represented by straight lines. The solution 'trajectories' are those parts of the phase portraits which flow between the wall boundary conditions. The phase portraits (which are symmetric around $\phi=0.5$ for a symmetric blend if $\lambda=0$ ) are distorted by the Lagrange multiplier $\lambda \neq 0$, which is a chemical potential. Suitable choices of both $\lambda$ and the Hamiltonian constant $\mathcal{H}$ (these sensitive parameters will be given to six decimal places) are necessary to produce phase equilibria trajectories of specified depth $d$ and average composition $\bar{\phi}$. Coexisting solutions must have the same depth $d_{A}=d_{B}$, which for the same $\lambda$ are generated with different values of $\mathcal{H}$ i.e., $\mathcal{H}_{A} \neq \mathcal{H}_{B}$ (since for $\chi>\chi_{W}$, there are several trajectories corresponding to profiles of the same depth, which can be obtained by producing phase portraits with different values of $\mathcal{H}$ and the same value of $\lambda$ ).

The phase portraits themselves can provide significant insight, as the evolution of trajectories can be tracked graphically as depth, temperature and wall interaction parameters change. Most importantly, we must understand that the 


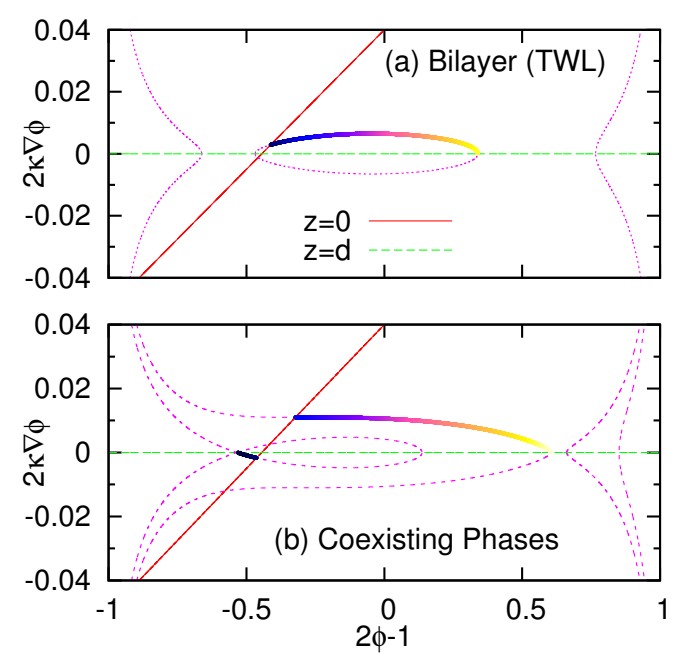

Figure 4.13: 1D phase portraits for equilibria in Fig. 4.12 (asymmetric walls). (a) Metastable bilayer (TWL) state $(\lambda=-0.000113, \mathcal{H}=0.001332)$, with two crosses of the $z=0 \mathrm{BC}$ along the flow; (b) Coexisting phases $\left(\lambda=-\mu_{\text {sim }}=-0.000367\right)$ : A-rich phase $\left(\mathcal{H}_{A}=0.001618\right)$ and (c) B-rich phase $\left(\mathcal{H}_{B}=0.001404\right) . \phi_{d}\left(\phi_{0}\right)$ of all three phases are rather distinct (similar).

ends of the trajectories are always pinned to the boundary conditions (BCs), even out of equilibrium, as shown in section 4.4.2). This is why bifurcation of the profile at the walls is inherent in lateral phase separation. I will denote the profile value of the TWL at the $z=0$ wall by $\left(\phi, 2 \kappa \nabla_{z} \phi\right)_{0}^{T W L}$, and similarly for other cases. I have coloured (shaded) the trajectories to match the colour range for $\phi$ shown in the final laterally segregated film of the simulation figures, to which the phase portraits correspond.

\subsubsection{One surface field}

Figs. 4.10, 4.12 and 4.14 are snapshots from $2 \mathrm{D}$ simulations of films with $\chi>\chi_{W}$ for $\chi=0.026, \chi=0.023$, and $\chi=0.022$ respectively, for asymmetric films of depth $d=20.1$, showing direct observations of lateral phase separation via a transient wetting layer. Time increases from sub-figure (a) to (d). In all cases the film first evolves into a 'bilayer' (vertically stratified profile), which is the TWL, and this bilayer subsequently breaks up into lateral segregated phases. Figs. $4.11,4.13$ and 4.15 show $1 \mathrm{D}$ phase equilibria $(d=20.1)$ in Hamiltonian phase space for the phases that form in Figs. 4.10, 4.12 and 4.14 respectively.

\section{Instability of a transient wetting layer}

Figs. 4.10, 4.12 and 4.14(a) all show a bilayer with a B-rich (A-rich) phase coating the B-attracting (neutral) wall, respectively, and an interface separating these phases [40] (for comparison, Figs. 4.4 and 4.5 are both bilayer profiles, whilst the profile of figure 4.3 is a monolayer with positive adsorption of $\mathrm{B}$ at the B-attracting wall at $z=0$ ). As $\chi$ increases, the bilayer interface sharpens (for $\chi<\chi_{W}$, this interface is rather diffuse, and the bilayer is stable against 


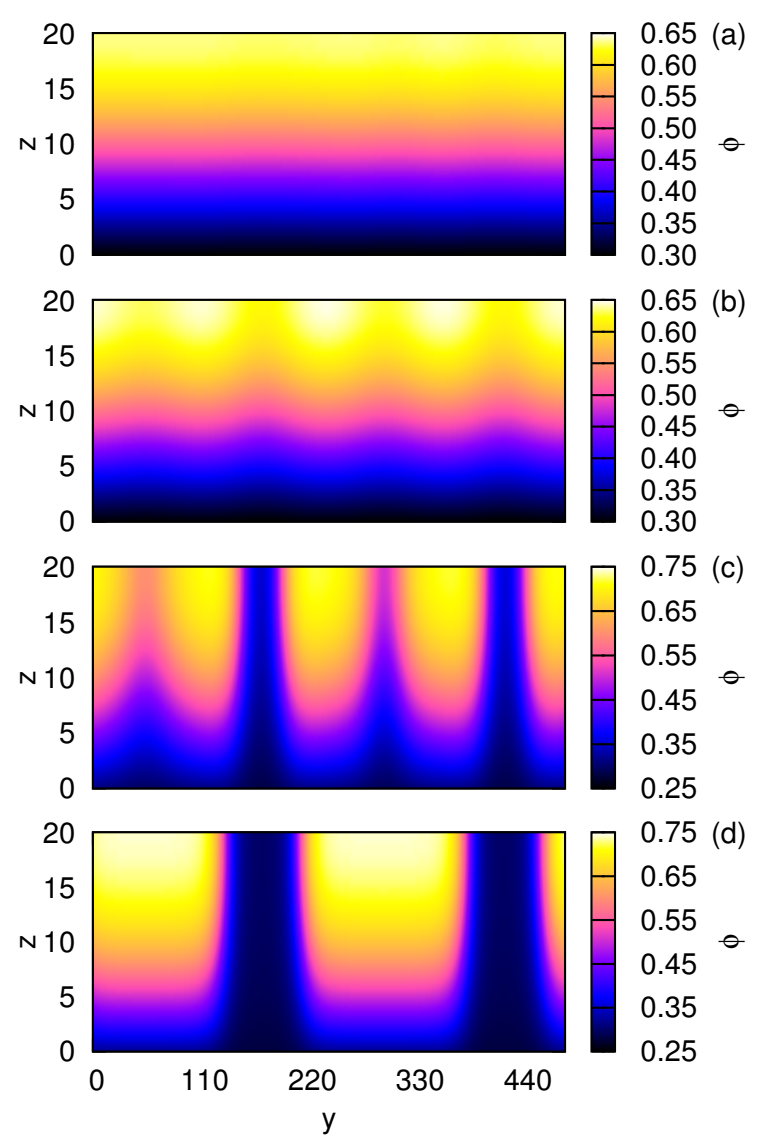

Figure 4.14: Simulation snapshots for $\chi=0.022\left(\chi>\chi_{W}\right), d=20.1$ and asymmetric walls $\left(\Delta z=0.56, \Delta \tau=0.25 \times 10^{-5}\right)$. (a) metastable bilayer state $(\tau=100)$; (b) late stages of bilayer state with visible change of $\phi$ at the $z=d$ wall $(\tau=1000)$; (c) merging of adjacent growing A-rich lateral phases at around $y \approx 50$ and $y \approx 300(\tau=1800)$; (d) laterally segregated coexisting states $(\tau=4000)$.

lateral phase separation, since a configuration of coexisting phases no longer has a lower free energy than the bilayer phase). Figs. 4.11, 4.13 and 4.15(a) show, in phase space, the independently existing solution of lowest free energy (a bilayer), $\lambda$ having been chosen to ensure $\bar{\phi}=1 / 2$. The average chemical potential of the bilayer state in Figs. 4.10, 4.12 and 4.14 (the average is over all grid cells) confirms that the films are in the $1 \mathrm{D}$ metastable bilayer states shown in the phase portraits of Figs. 4.11, 4.13 and 4.15(a) (e.g. for $\chi=0.026,\left\langle\mu_{\text {sim }}>=0.000112\right.$, whilst $-\lambda=0.000119$ for the calculated profile, it is hopefully obvious that there are no other equilibria that the films could be in at this stage).

Figs. 4.10, 4.12 and 4.14 show that lateral inhomogeneities in the bilayer state continue to grow with time and the interface separating the phases of the bilayer becomes distorted (subfigure (b)). Any distortion of the interface appears to correspond to lateral inhomogeneities which have appeared at the confining walls, most notably at the neutral wall at $z=d$. The average chemical potential remains approximately that of the bilayer during this distortion, and only when the interface appears to break up does the average chemical potential begin to rapidly change, indicating that the film is now in the process of leaving its long- 


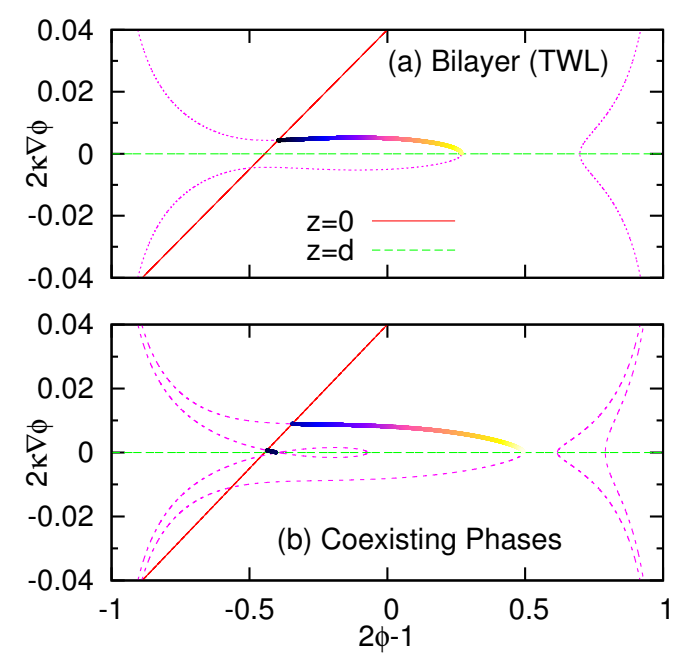

Figure 4.15: 1D phase portraits for equilibria in Fig. 4.14 (asymmetric walls). (a) Metastable bilayer (TWL) $(\lambda=-0.000184, \mathcal{H}=0.001580)$; (b) Coexisting phases $\left(\lambda=-\mu_{\text {sim }}=-0.000333\right)$ : A-rich phase $\left(\mathcal{H}_{A}=0.001747\right)$ and B-rich phase $\left(\mathcal{H}_{B}=\right.$ $0.001589)$. The B-rich trajectory shows $\phi_{B} \equiv 1-\phi$ increases towards the $z=0$ wall, which was not the case for Figs. 4.10 and 4.12) $\phi_{d}\left(\phi_{0}\right)$ of all three phases are rather distinct (similar).

lived metastable equilibrium. At later times (subfigure (c)) the interface breaks up: figure 4.10(c) shows the interface moments after a column of B-rich material reaches the $z=d$ surface; figure $4.12(\mathrm{c})$ shows the interface just prior to break up, showing significant variations in $\phi_{d}$; and figure 4.14(c) shows the film after the break up of the interface as some lateral phases merge to reduce interfacial energy. It can be seen that points where the interface touches down on the walls and where the lateral phases develop from are exactly the same points where the initial lateral variations at the walls took place, and in fact it appears that the Arich phases are growing from the neutral wall at $z=d$. The final states (subfigure (d)) of Figs. 4.10, 4.12 and 4.14 show the film in the laterally segregated state, and it is clear that the lateral phases have formed exactly where the initial lateral variations at the walls took place. This strongly suggests that the wall-blend interactions are controlling the dynamics of lateral phase separation.

For $\chi>\chi_{W}$, a bilayer is unstable with respect to a laterally segregated film due to having a greater free energy as discussed in chapter 3 (again, this is really the definition of the wetting temperature), and it is clear that the result carries across to $2 \mathrm{D}$ (although a $1 \mathrm{D}$ consideration may not be able to predict the wetting temperature, since it cannot account for the lateral interfaces between coexisting phases, which has an associated energy cost). I conclude that the transient wetting layer, which initially forms due to preferential attraction by the confining walls, breaks up because it is metastable with respect to the laterally segregated state. The intrinsic instability of the transient wetting layer as a whole is different from an instability in the interface between the vertically segregated phases of the bilayer. Note the similarities in the breakup of the bilayer in my simulations and the schematics of figure 4.1: the interface of the bilayer distorts 


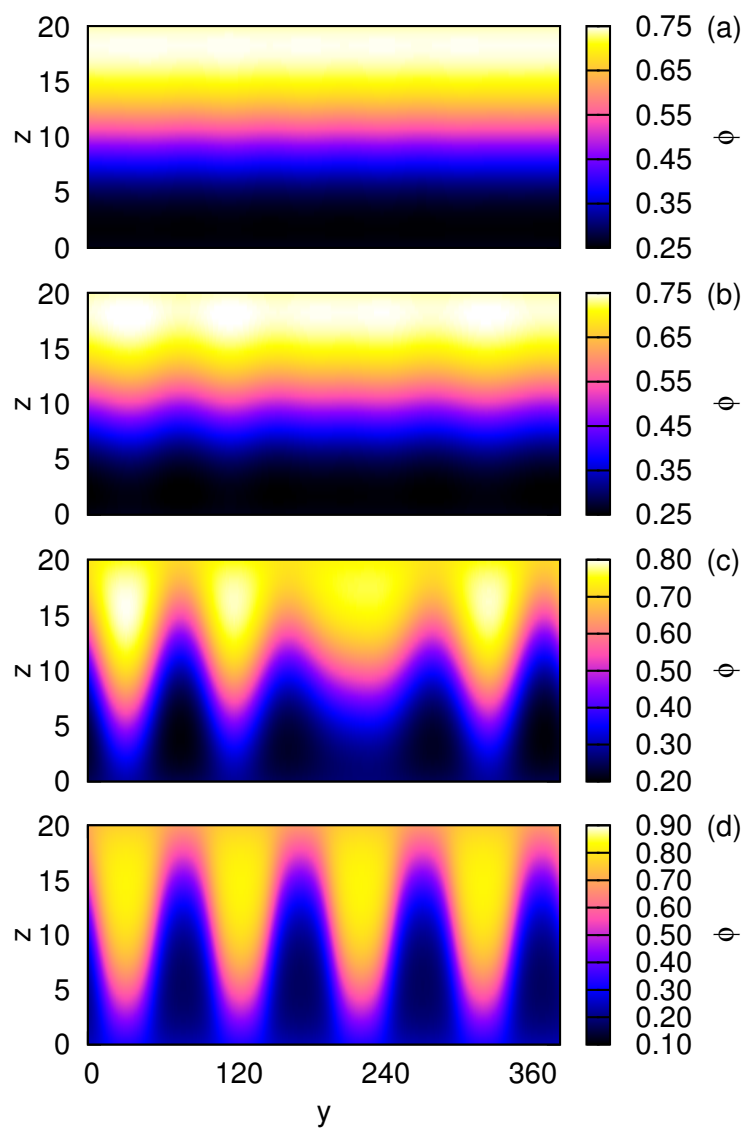

Figure 4.16: Simulation snapshots for $\chi=0.026\left(\chi>\chi_{W}\right), d=20.1$ and antisymmetric walls $\left(\Delta z=0.50\right.$ and $\left.\Delta \tau=1.0 \times 10^{-5}\right)$. (a) metastable bilayer state $(\tau=150)$; (b) rupture of coexisting A-rich and B-rich layers of bilayer, coupled with bifurcation of $\phi$ at both walls $(\tau=1300)$; (c) continued rupture of layers distorts interface towards the walls, since the wall boundary conditions must be satisfied $(\tau=2500)$; (d) the boundary conditions of the laterally segregated states are met $(\tau=4500)$.

in a practically identical way, and yet my simulations clearly don't include solvent gradients or hydrodynamic mechanisms. The bilayer breaks up only because it is metastable. This is discussed further in section 4.6.2.

\section{Explaining the dynamics}

The phase portraits Figs. 4.11, 4.13 and 4.15 can be used to explain the dynamics of the film evolution. Figs. 4.11, 4.13 and 4.15(b) are phase portraits of the A-rich and B-rich laterally coexisting phases respectively, calculated using the chemical potentials extracted from the simulations $\lambda=-\mu_{\text {sim }}$ when the film has achieved a static laterally segregated state. The phase portraits describe the profiles from the simulations exactly, and clearly show how the equilibria evolve as $\chi$ is changed, including the increased homogeneity of the B-rich phase as $\chi=0.026 \rightarrow 0.023$ (the trajectory becomes 'shorter' in phase space) and a qualitative change in the B-rich solution for $\chi=0.023 \rightarrow 0.022$ as the B-attracting wall becomes richer in $\mathrm{B}$ material than elsewhere in the B-rich phase. 


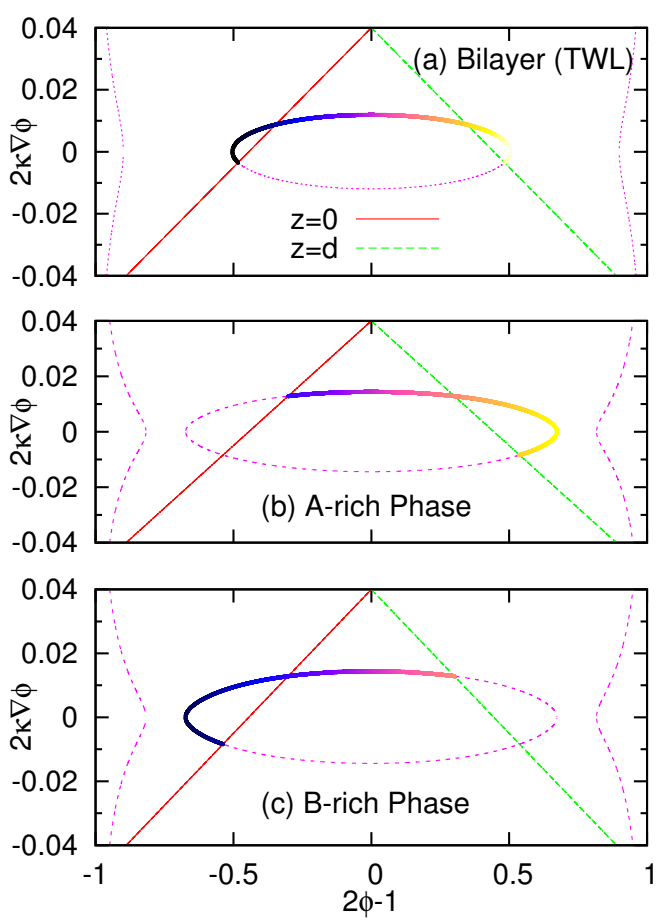

Figure 4.17: 1D phase portraits for equilibria in Fig. 4.16 (antisymmetric walls). (a) Metastable bilayer (TWL) $(\lambda=0, \mathcal{H}=0.000752)$; Coexisting phases $\left(\lambda=-\mu_{\text {sim }}=0\right)$ : (b) A-rich phase $\left(\mathcal{H}_{A}=0.000899\right)$ and (c) B-rich phase $\left(\mathcal{H}_{B}=0.000899\right)$. Since $\lambda=0$ the phase portraits are symmetric around $\phi=0.5$. The bilayer trajectory passes through $\nabla \phi=0$ near each BC, whilst the A-rich (B-rich) trajectories pass through $\nabla \phi=0$ only near the A-attracting (B-attracting) wall BC at $z=d(z=0)$.

For $\chi=0.022$, figure 4.15 shows that the trajectory of the TWL passes through each boundary condition only once (although the Hamiltonian flow crosses each boundary condition twice, in the region of interest) and the same is true for the A-rich and B-rich trajectories, which flow between crosses similar to that of the TWL. In this case, we see that $\left|\phi_{0}^{A}-\phi_{0}^{B}\right|<<\left|\phi_{d}^{A}-\phi_{d}^{B}\right|$ (the values of $\phi_{d}$ for the coexisting phases are more different from each other than the values of $\phi_{0}$ for the coexisting phases) and $\left|\phi_{0}^{T W L}-\phi_{0}^{A}\right|<<\left|\phi_{d}^{T W L}-\phi_{d}^{A}\right|$, which means that for the A-rich phase to form from the TWL, $\phi_{d}$ must change by much more than $\phi_{0}$. As figure 4.14 shows, lateral phase separation happens as the A-rich lateral phases appear to grow from the $z=d$ wall. For $\chi=0.023$, even though the B-rich trajectory exists on a different region in the phase space (the closed tear-shaped loop in figure 4.13(b)) such that the 'bulk' of the profile is slightly different, figure 4.12 shows that the lateral phases still appear to grow from the $z=d$ surface, since the same arguments as for the previous case can be made. For $\chi=0.026$, figure 4.11 shows that the TWL trajectory crosses each boundary condition twice, and each coexisting phase flows between a different cross of the flow with the boundary conditions. $\phi_{0}$ and $\phi_{d}$ for both the A-rich and B-rich phases differ much more from $\phi_{0, d}^{T W L}$ than in the case of $\chi=0.022$. This is especially true of $\phi_{0}^{A}$. Figure 4.10 shows that the break up of the interface is due to significant variations in $\phi$ at both confining walls, the largest variations in $\phi_{0}^{T W L}$ 


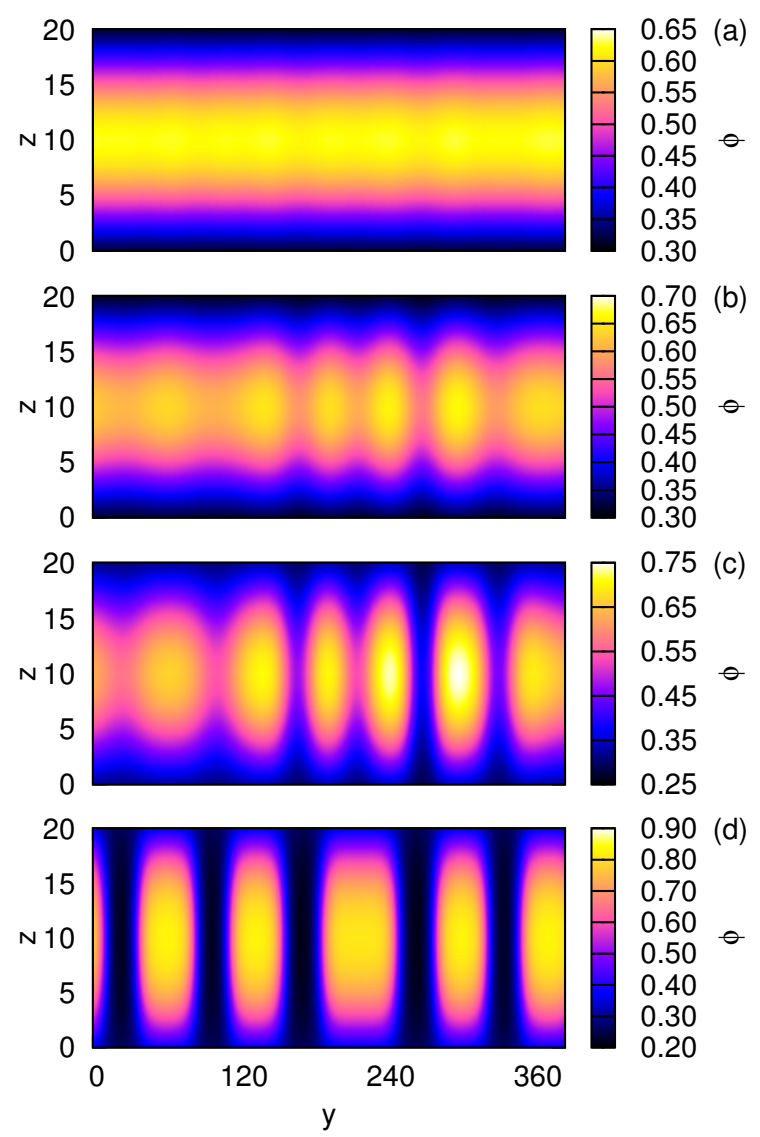

Figure 4.18: Simulation snapshots for $\chi=0.026\left(\chi>\chi_{W}\right), d=20.1$ and symmetric walls $\left(\Delta z=0.50, \Delta \tau=1.0 \times 10^{-5}\right)$. (a) metastable trilayer state $(\tau=50)$; (b) A-rich (central) layer begins to phase separate, causing corresponding changes in $\phi$ throughout the trilayer $(\tau=300)$; (c) rupture of the A-rich layer once bifurcation of $\phi$ at the walls is sufficient $(\tau=450)$; (d) laterally segregated coexisting states $(\tau=1000)$.

of the bilayer being precisely where the columns of A-rich phase form. This is expected from inspection of figure 4.11, which shows that $\phi_{0}^{T W L}$ and $\phi_{0}^{B}$ are still fairly similar, but $\phi_{0}^{A}$ of the A-rich phase is significantly different from both those values. The column of B-rich phase reaches the $z=d$ surface when $\phi_{d}$, which had been gradually change during the interface distortion, suddenly undergoes a quick transient $\phi_{d}^{T W L} \rightarrow \phi_{d}^{B}$ as the interface appears to reach the surface and break. The phase portraits thus offer practical insight into the dynamics of the break-up of the transient wetting layer.

The simulations of Figs. 4.10, 4.12 and 4.14 seem to show that the breakup of the bilayer state proceeds from the neutral wall at $z=d$. Since the profiles are pinned to the surface boundary conditions at all times, lateral phase separation clearly requires that the single value of the volume fraction at each wall for the TWL must undergo a bifurcation into two values for the laterally coexisting phases: $\phi_{0, d}^{T W L} \rightarrow \phi_{0, d}^{A}, \phi_{0, d}^{B}$, the bifurcation at the $z=d$ wall being much more pronounced for asymmetric films (clearly, due to the interface between the coexisting phases, there are more than two values at the wall, but it is much simpler to discuss the coexisting states in terms of the $1 \mathrm{D}$ phase equilibria that 


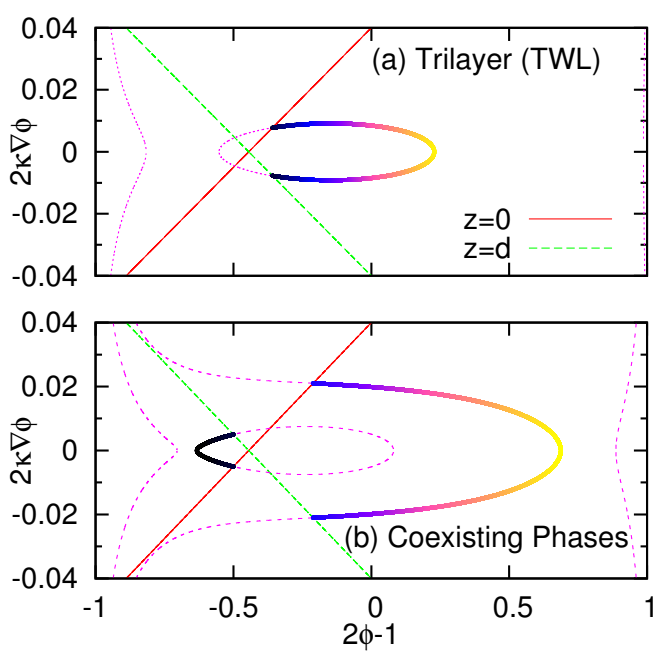

Figure 4.19: 1D phase portraits for equilibria in Fig. 4.18 (symmetric walls). (a) Metastable trilayer (TWL) $(\lambda=-0.000750, \mathcal{H}=0.000968)$; (b) Coexisting phases $\left(\lambda=-\mu_{\text {sim }}=-0.001204\right)$ : A-rich phase $\left(\mathcal{H}_{A}=0.001922\right)$ and B-rich phase $\left(\mathcal{H}_{B}=\right.$ $0.001092)$. In all cases, $\nabla \phi=0$ is located exactly in the centre of the film.

appear in the simulations). Note that this bifurcation at the surface is technically $\left(\phi, 2 \kappa \nabla_{z} \phi\right)_{0, d}^{T W L} \rightarrow\left(\phi, 2 \kappa \nabla_{z} \phi\right)_{0, d}^{A},\left(\phi, 2 \kappa \nabla_{z} \phi\right)_{0, d}^{B}$ but it is sufficient here to discuss only $\phi_{0, d}$ (which can be experimentally measured). The distortion of the interface in the TWL is coupled to phase separation at the walls due to the boundary conditions enforced by the walls, causing the distortion of the bilayer interface as the films evolve towards laterally coexisting equilibria. I have not seen this surface bifurcation ${ }^{25}$ mechanism discussed elsewhere.

\subsubsection{Two surface fields}

Figure 4.16 shows snapshots from simulations of a polymer blend between antisymmetric confining walls. As in the case of asymmetric confinement, a bilayer (TWL) first forms which subsequently breaks up into laterally segregated phases. The Hamiltonian flow containing the bilayer trajectory, shown in figure 4.17(a), is very similar to the flow in figure 4.11(a) for an asymmetric film. The phase portrait of figure 4.17(a) shows that the TWL trajectory passes through $\nabla \phi=0$ (stationary points) near each wall, with a corresponding maximum in $\phi_{A} \equiv \phi$ $\left(\phi_{B} \equiv 1-\phi\right)$ near the A-attracting (B-attracting) wall. The simulation in figure 4.16 shows that the distortion of the interface in the bilayer appears to be caused by growing lateral inhomogeneities at these stationary points, although the wall boundary conditions ensure that lateral inhomogeneities simultaneously grow at the walls. However, it certainly appears that rupture of the film proceeds from the stationary points near the surfaces. Inspection of the phase portraits of

\footnotetext{
${ }^{25}$ Surface Bifurcation: mechanism explaining the dynamics of lateral phase separation via a transient wetting layer, describing how the surface values $\left(\phi, 2 \kappa \nabla_{z} \phi\right)$ of the TWL divide into two values that evolve towards those for laterally coexisting equilibria, whilst honouring the surface boundary conditions at all times throughout the entire process.
} 
figure 4.17 shows that in order for the film to laterally separate, not only does the volume fraction at the confining walls have to undergo bifurcation, but one of the stationary points needs to disappear. For the A-rich (B-rich) phase, the stationary point near the A-attracting (B-attracting) wall is preserved and enriched in A-material (B-material), whilst the other stationary point disappears exactly where the B-rich (A-rich) phase forms. The enrichment and removal of a stationary point happens at the same depth, so we see lateral phase separation occurring at the stationary points, causing a distortion of the interface towards the walls where the stationary points disappear. Also, for lateral phase separation to occur, the required change in the profiles at the walls is much less than the change required at the stationary points. Whilst it is clear that lateral phase separation at the walls is inherent in this process, as film thickness is increased the stationary points in the trajectories can pass arbitrarily close to fixed points in the phase space (these fixed points are located at $\nabla_{z} \phi=0$ between the gaps in the Hamiltonian flows; see chapter 3 section 3.3.4), meaning that the amount of the film profile constituting a stationary point can become arbitrarily thick.

It should be noted that the final state of figure 4.16 does indeed contain the laterally coexisting phases shown in the phase portraits of figure 4.17, and does not simply show an oscillatory interface. Lateral phase separation in antisymmetric films is in fact the transition from a delocalised interface (bilayer) to an interface bound to one of the walls (laterally coexisting phases) [40, 36]. In section 4.6.1 figure 4.21, I show that the lateral domains of figure 4.16 will evolve into wider trapezoidal phases [65] when continuous noise is included in the simulation.

For a film between symmetric walls, shown in figure 4.18, the TWL that forms first is actually a trilayer structure. It is clear that lateral phase separation occurs when the central layer ruptures. The phase portraits of figure 4.19 show that this rupture again occurs at a stationary point in the profile, where $\nabla_{z} \phi=0$. The rupture of the central layer preserves the A-rich stationary point of the trilayer for the A-rich phase, and columns of B-rich phase form in the depleted regions caused by the enrichment of the A-rich stationary points. Although lateral phase separation via a transient wetting layer is usually referring to the break up of a bilayer film, this case shows that although bifurcation of the volume fraction at the confining walls is necessary for lateral phase separation to occur (and will occur as phase separation occurs anywhere in the transient wetting layer, due to the wall boundary conditions), the break up of the TWL may primarily proceed via growth of lateral inhomogeneities in some other part of the film.

\subsubsection{Bypassing the wetting layer}

If the dynamics of lateral phase separation via a transient wetting layer are ultimately controlled by wall-blend interactions (via boundary conditions enforced by the walls), then it should be possible to manipulate the dynamics in the film by attempting to control behaviour at the confining walls. Investigating this possibility led to a possible method that may prove useful in obtaining laterally segregated films, as opposed to a TWL or a structure in-between a TWL and a laterally segregated film. In the case of the asymmetric confinement (B-attracting 

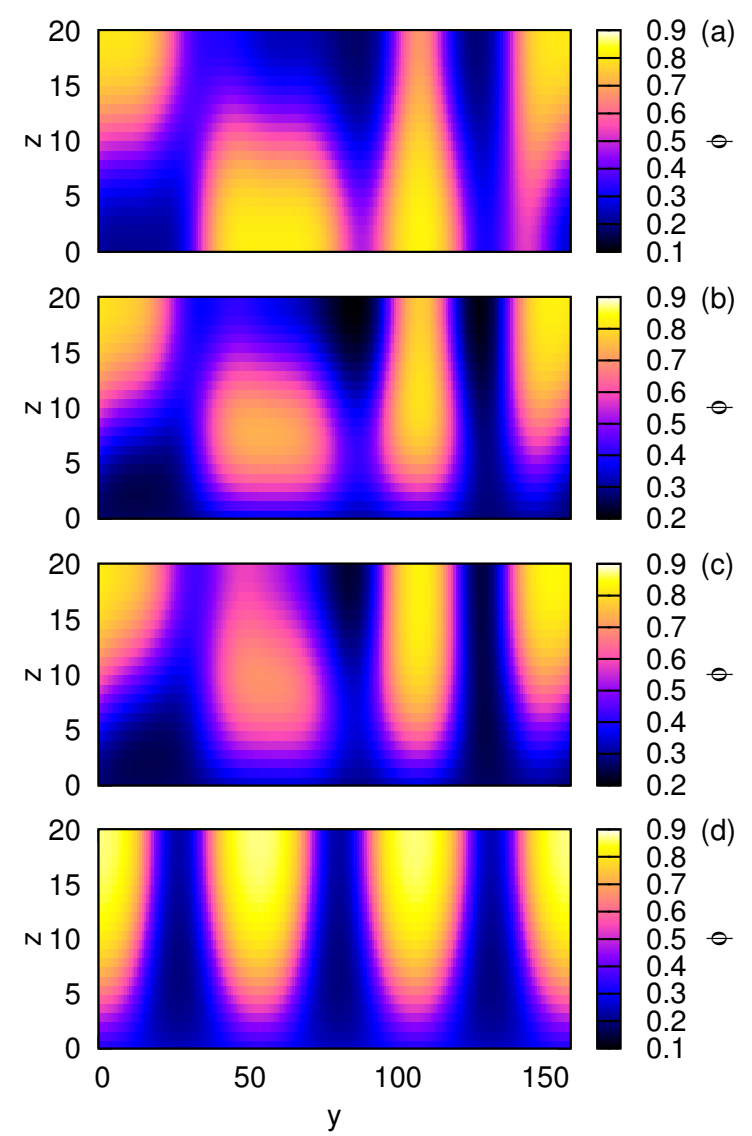

Figure 4.20: Simulation snapshots for $\chi=0.026\left(\chi>\chi_{W}\right), d=20.1$ and asymmetric walls, $\left(\Delta z=0.84, \Delta \tau=1.0 \times 10^{-4}\right)$. Both walls are initially neutral, then the $z=0$ wall is 'turned on' (film becomes asymmetric) at $\tau=160$. (a) phase separation with domains aligned to walls to satisfy BCs requiring $\nabla \phi=0(\tau=150)$; (b) moments after $z=0$ wall is 'turned on' ( $\tau=160)$; (c) rapid evolution towards the coexisting states of the asymmetric film $(\tau=170)$; (d) laterally segregated state $(\tau=300)$ is reached an order of magnitude faster than via a transient wetting layer.

wall at $z=0$, neutral wall at $z=d$ ), if the B-attracting wall could be 'turned off' temporarily to become a neutral wall, then we should suppose for a near critical mixture that a transient wetting layer will not form, and the film should phase separate more generally. This is because there should be no mechanism via which vertical phase separation in particular should proceed (in a non-critical blend $\phi \neq 1 / 2$, layering parallel to the walls may sometimes still occur [52] even with no surface fields, simply as a way to reduce the amount of interface between the majority and minority component of the blend, and if the simulations were $1 \mathrm{D}$ then of course vertical phase separation would in fact be the only route). If the $B$-attracting wall was 'turned on' at some later time after 'enough' phase separation has already happened, the laterally segregated phases may be obtained without having formed a bilayer first.

Figure 4.20 demonstrates that this may work in practice. When both walls are 'turned off' (subfigure (a)), we see phase separation into domains with $\nabla_{z} \phi=0$ (required for neutral walls, as can be inferred readily from equations (4.8) and 
(4.9)). There are (in terms of 1D equilibria) two values of $\phi$ at each wall. The $z=0$ wall is 'turned on' to attract B-material at $\tau=160$ in subfigure (b) (asymmetric wall configuration) and there is immediate preferential attraction of B-material to the $z=0$ surface. However, $\phi_{0}^{A}$ and $\phi_{0}^{B}$ have been obtained, rather than $\phi_{0}^{T W L}$. The TWL has been avoided and the film can evolve directly towards the laterally segregated state, shown in the subfigure (d), and lateral segregation is achieved an order of magnitude faster than via a TWL. In solvent evaporation processes, such as spin coating, it might be possible to use this mechanism by choosing a solvent to adjust the wall-blend interactions, thus allowing for greater control over film evolution and final morphology.

\subsection{Discussion}

\subsubsection{Random Noise}

The absence of a random noise term in my simulations, although maintaining clarity in the results, is why many lateral phases don't become more macroscopic at very late times (e.g. figure 4.16 does not show the wide trapezoidal-shaped coexisting phases expected at long times [65] although such trapezoidal-shaped phases are simply wide versions of the phases shown), although this consideration is of little practical relevance in many experiments including solvent evaporation, for example the spin-coating process described in section 4.1.2. Figure 4.21 shows a simulation for antisymmetric walls in which a continuous noise was included throughout the simulation. This random noise consisted of random thermal currents $J^{*}$, selected from a Gaussian distribution with mean zero and amplitude/width 0.01 . This gave an additional contribution to the diffusion equation 4.21 of $\nabla \cdot J^{*}(z, y, t)$, as in ref [65] and in chapter 1 equation (1.65) on Cahn-Hilliard-Cook theory:

$$
\frac{\partial \phi}{\partial t}=M \nabla^{2} \mu+\nabla \cdot J^{*}
$$

Using random thermal currents is more physical than simply applying some random change in volume fraction at each time step: currents guarantee material conservation, both globally and locally (the local movement of material in and out of grid cells is consistent).

Figure 4.21 shows that a bilayer still forms and breaks up via the mechanism I have already presented. There are smaller scale lateral variations at the surface initially, and wider domains form at later times. Figure 4.21 appears to reproduce a contact angle appropriate for an antisymmetric film. I did not find that the $\mathrm{A}(\mathrm{B})$-attracting surface is coated by $\mathrm{B}(\mathrm{A})$-rich material, as is sometimes suggested by schematic representations (the phase portraits of figure 4.17 support this; the $\mathrm{A}(\mathrm{B})$-rich trajectories must pass through $\phi=0.5$ near the $\mathrm{B}(\mathrm{A})$-attracting wall for antisymmetric films, which agrees with the idea that these coexisting phases have an interface 'bound' to one of the walls [40, 36]). 

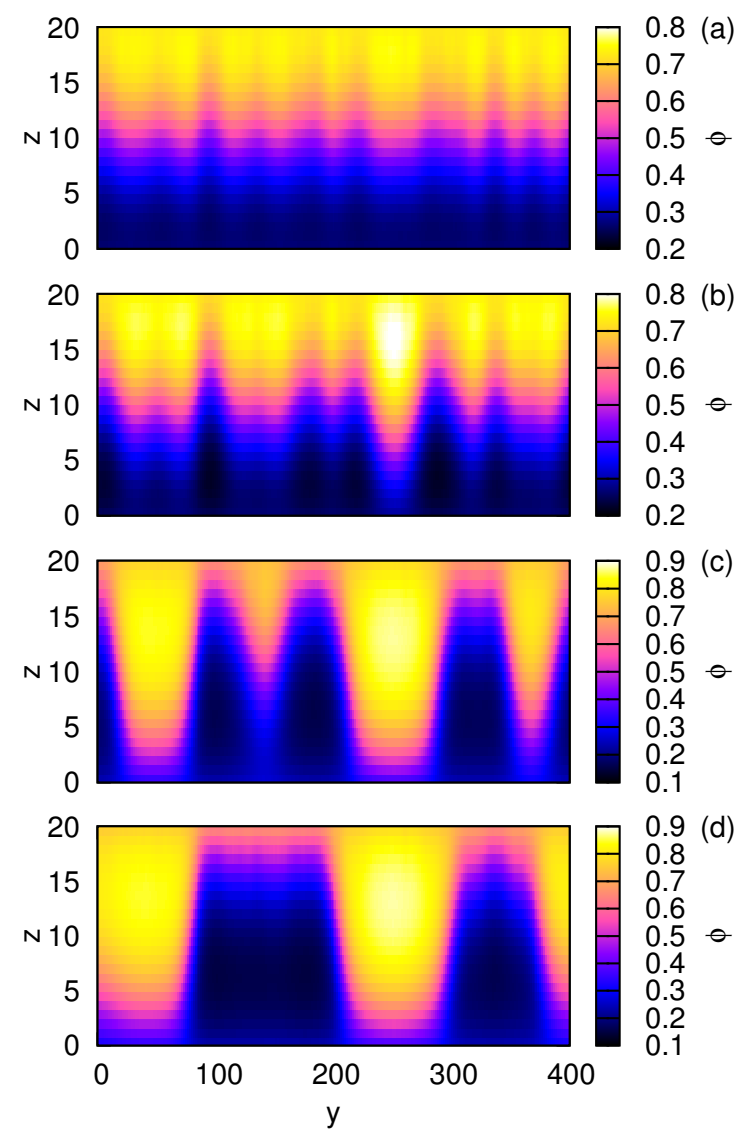

Figure 4.21: Simulation snapshots for $\chi=0.026\left(\chi>\chi_{W}\right), d=20.1$ and antisymmetric walls, $\left(\Delta z=0.84, \Delta \tau=1.0 \times 10^{-4}\right)$. Random noise is included at every time step. (a) The bilayer interface is heavily distorted $(\tau=25)$; (b) lateral A-rich phase begins to form as the A-rich layer of the bilayer ruptures $(\tau=125)$ (c) A-rich phase retreats from B-attracting wall $(z \approx 150)$ and neighbouring A-rich phases grow $(\tau=2225)$; (d) Macroscopic trapeziodal domains form at longer times $(\tau=5000)$. These phase equilibria match those in Figs. 4.16 and 4.17.

\subsubsection{Solvent Evaporation}

Many practical applications using polymer films involve solvent evaporation, such as the spin casting process discussed in section 4.1.2. Here I discuss the implications of my results for experiments involving solvent evaporation. I do this because the model reproduces and explains the way in which the transient wetting layer breaks up without needing to include solvent. Given this, and evidence (see references below) that the rate of solvent evaporation has an effect on whether a bilayer will break up during the spin casting process ends (i.e. before all solvent has been removed from the film), I suggest that solvent may influence lateral phase separation in several ways:

1. fast solvent evaporation provides lateral inhomogeneities in the transient wetting layer due to a less even distribution of solvent (this will depend on the rate of evaporation and the mobility of the solvent). This could provide a kinetic route to a laterally segregated film by promoting lateral 
inhomogeneities in the already unstable TWL, which could encourage the formation of a laterally segregated film which is sometimes not achieved: my simulations and frozen out-of-equilibrium states found in experiments [59] show strong resemblances;

2. solvent evaporation allows phase separation to initiate at the top surface, where the solvent concentration is lowest and therefore where the film is likely to first enter the two-phase region, this phase separation then proceeding downwards into the film [66]. In this case, the solvent in the 'bulk' of the film keeps it in the one-phase region and prevents the formation of a TWL, and when the film enters the two-phase region the existing lateral inhomogeneities mean that the film remains laterally segregated, which is similar to my discussion in section 4.5.3 (although my simulations did not include solvent, the equilibria of ternary polymer-polymer-solvent films are analogues of equilibria for binary polymer-polymer films [42]);

3. overall reduction of solvent in the film induces phase separation by taking the film into the two-phase region, and lateral phase separation occurs in stages that appear to match the simulations, with high, medium, and low solvent concentrations corresponding to a bilayer, a bilayer with a distorted interface, and a laterally segregated film, respectively [61]. Higher solvent concentrations mean that the film is more miscible and closer to the onephase region, therefore the time scales on which one might expect a bilayer to break up may be expected to be longer.

Although a Marangoni-like instability has been suggested to explain the distortion of the bilayer interface prior to lateral phase separation $[62,61,41]$, in a manner like in figure 4.1, I have shown that the intrinsic instability of the bilayer and surface bifurcation is sufficient to cause distortion of the interface in a similar if not near-identical way. Therefore we should consider that the phase equilibria of polymer films and the surface bifurcation mechanism I have presented here might be responsible for the film evolution seen in many experiments. Of course this is not to say that solvent-gradients and hydrodynamic mechanisms of material transport are not also extremely important.

\subsection{Summary}

I have derived a diffusion equation describing a binary polymer blend confined between two preferentially attracting walls/surfaces. I compared the phases produced in the simulations with profiles calculated using a 1D Hamiltonian phase portrait method to show that the diffusion equation correctly reproduces continuum behaviour and that all of the equilibria that arise in $2 \mathrm{D}$ are simply $1 \mathrm{D}$ coexisting phases, existing in 2D under an altered chemical potential due to the lateral interfaces between these coexisting phases. I also showed the film profile is pinned at the film walls by effective boundary conditions.

I have identified the dynamics of lateral phase separation via a transient wetting layer for several wall-blend interaction configurations, showing that distortion 
of the interface in the transient wetting layer is coupled to changes in the film profile at the walls. For a film with one preferentially attracting wall and one neutral wall, the growth of lateral inhomogeneities at the confining walls causes distortion and breakup of the interface in the transient wetting layer. For films with two preferentially attracting walls, phase separation appears to primarily proceed from elsewhere in the film, but there are always corresponding inhomogeneities growing at the walls. The instability of a transient wetting layer (below the wetting temperature) as a whole is not the same as an instability in the interface of the transient wetting layer; in all cases I have studied, the growth of lateral inhomogeneities at the walls limits and dictates the dynamics.

I have explained the dynamics of lateral phase separation via a transient wetting layer with a surface bifurcation mechanism: the pinning of the profile at the film walls by effective boundary conditions imposed by the film surfaces means that the film must undergo bifurcation of the profile at the walls in order to laterally phase separate into coexisting phases. The distortion of the interface in the wetting layer coincides with phase separation at the surfaces. Since these results should also extend to ternary blends, I discussed how solvent evaporation may assist the proposed mechanism. As far as I am aware, this is the first time that a mechanism has been suggested that accounts for the particular way in which lateral phase separation via a transient wetting layer occurs, without requiring solvent evaporation and/or non-diffusive flow of material (i.e. hydrodynamics).

In the next chapter, I introduce a 3D model of coupled phase separation and surface roughening, whereby the depth profile of the film evolves via a dewetting mechanism. This allows for an investigation of how surface roughening, discussed briefly in chapter 3, and lateral phase separation, discussed in this chapter, may be coupled, resulting in particular pattern formations in polymer-blend thin films. 



\section{A 3D Model OF \\ Phase Separation COUPLED TO Surface Roughening}

I introduce a model for thin films of multicomponent fluids which, by successfully including a general vertical dependence of the composition, can account for lateral and vertical phase separation, preferential component attraction at both surfaces, and surface roughening. I demonstrate that surface roughening couples to phase separation, study stages of surface roughening in films undergoing lateral phase separation via a transient wetting layer, and discuss pattern formation for different surface-blend interaction regimes. This work was published in my fourth paper "Pattern Formation in Polymer Blend Thin Films: Surface Roughening couples to Phase Separation" [67].

5.1 Introduction . . . . . . . . . . . . . . . . . . . . . . . . . . 108

5.2 A 3D Model . . . . . . . . . . . . . . . . . . 111

5.3 Application to polymer-blend thin films . . . . . . . . . . . . 116

5.4 Results and Discussion . . . . . . . . . . . . . . . . . . 118

5.5 Summary . . . . . . . . . . . . . . . . . . . . 125 


\subsection{Introduction}

The work in this chapter resulted from my attempts to build a 3D model of a polymer-blend thin film which could lend itself to simulations of simultaneous phase separation and surface roughening. My work was an extension of that of Clarke [68], in which a fluid film with no vertical variation of the composition (only a 2D lateral dependence of the composition) was modelled and used to investigate coupled phase separation and dewetting. Including this vertical dependence proved non-trivial, and was solved by using an a priori discretisation of the film.

By a way of including a general vertical dependence of composition, I could investigate for the first time the interplay between all of (i) both lateral and vertical phase separation; (ii) preferential attraction of the blend by both surfaces (which really requires a vertical dependence of the composition to be meaningful); and (iii) film height evolution (surface roughening). I applied the model to thin films of binary polymer blends, and used simulations of different surface-blend interaction regimes to investigate pattern formation, demonstrating that surface roughening couples to phase separation. For films undergoing lateral phase separation via a transient wetting layer, this resulted in distinct stages of roughening as the film evolved into and between states determined by the phase equilibria of polymer blend thin films.

\subsubsection{Relevant Literature}

Semiconducting polymer devices, such as photovoltaic films of binary polymer blends, generally rely on performance enhancements gained from phase separated morphologies to compete with traditional technologies. Whilst understanding phase separation assists in tailoring specific morphologies, polymer blend thin films are also prone to surface roughening, so it follows that an understanding of how phase separation couples to dewetting is of particular importance. Note that dewetting $^{26}$ in this context is meant in the sense of a fluid layer deweting a solid substrate: the fluid layer does not remain uniform in height, but tends towards the formation of droplets as regions of the substrate tend towards 'drying' (this does not necessarily mean that the fluid layer will breakup into droplets; it may lead to an undulating film surface etc.).

\section{Surface Roughening in polymer-blend films}

The interplay between phase separation and dewetting in domain formation in spin-cast polymer blend films (see chapter 4 for spin-casting) was highlighted in the seminal work of Walheim [59], and has been repeatedly observed in similar contexts, including: phase separation in thin films with symmetrically attracting surfaces $[69,70,71,72]$, lateral phase separation via a transient wetting layer [60, 61], and cases when lateral phase separation proceeded downwards from the film

\footnotetext{
${ }^{26}$ Dewetting: (disambiguation) process by which a fluid film will not uniformly coat a substrate, due to forces which cause areas of substrate to tend towards 'drying'; this can lead to isolated droplets of fluid, but more generally will cause some undulation of the fluid film surface.
} 


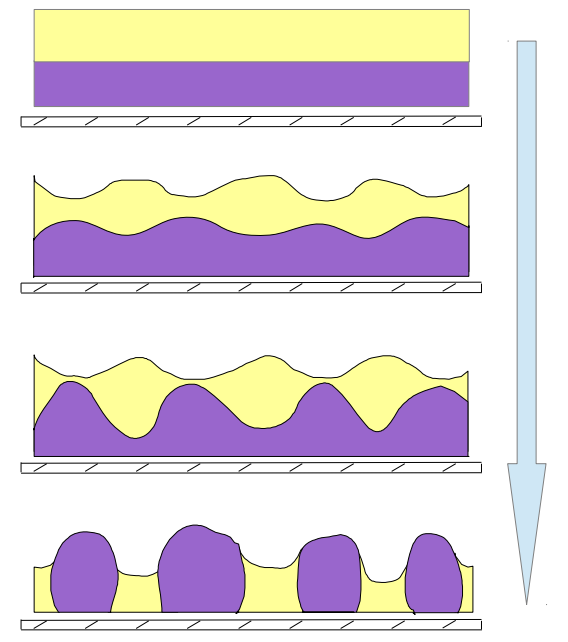

Figure 5.1: A fairly typical schematic of a film undergoing roughening in the course of lateral phase separation via a transient wetting layer: the laterally segregated structure and the surface roughening are intimately linked.

surface as solvent evaporated from the film [73]. Pattern formation in polymer blend thin films, in which surface roughening shadows the phase separated morphology, is so incredibly common that it is difficult to highlight particular studies, but the review articles of references $[74,75]$ are recommended. Figure 5.1 is a rough schematic of surface roughening that is fairly typical in the literature [59, 60 ], showing that roughening is associated with the lateral phase separation of a bilayer film (the particular morphology in this schematic is only an example).

Several general theoretical results have emerged, in particular that instabilities leading to dewetting can be triggered through the coupling of phase separation and height variation $[68,76]$ and that a binary component film will be less stable due to coupling of fluctuations of height and composition than if these fluctuations were not coupled [77]. These studies clearly indicate the importance of the coupling between phase separation and dewetting/surface roughening.

\section{Models of deformable fluid films}

A variety of models of multicomponent fluid films with a deformable upper surface have been investigated. The Clarke model, as it will be referred to in this chapter, utilised non-equilibrium thermodynamics based upon a free energy functional, demonstrating that phase separation generally couples to dewetting [68]: the Clarke model is explained more in section 5.1.2. A model based on the Navier-Stokes Cahn-Hilliard equations in the lubrication approximation showed that concentration gradients can create a roughened pattern that mirrors the underlying phase separation [78]. However, the film composition has no vertical dependence in these models, which means that a meaningful preferential surface attraction of blend components cannot be included. A vertical dependence has been included by way of two-layer models, including models with immiscible fluid layers $[79,80]$ and layers with a diffuse boundary for films with no preferential surface attraction [81]. A schematic of a two-layer model is given in figure 5.2. 


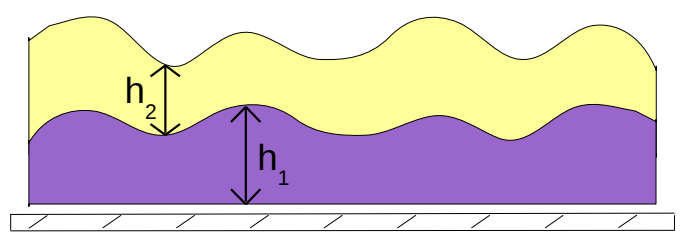

Figure 5.2: Representation of a two-layer model, which effectively consists of twoimmiscible fluid layers with a sharp interface between them. Both of the layers are deformable, such that layer $1(2)$ is described at $y$ by height $h_{1}(y)\left(h_{2}(y)\right)$, where $y$ is the direction parallel to the substrate.

A general vertical dependence of the film composition, which could allow vertical phase separation to occur during a simulation, is typically not included [74]. Films with a vertical dependence of the composition have been studied with regards to stability, but not simulated [82]. An exception is a model of surface roughening of polymer blend films, although this model is not based upon a dewetting film [83]. It is this lack of investigation into a general 3D model of a film which can undergo phase separation and dewetting/surface roughening which inspired the work in this chapter.

\subsubsection{The Clarke Model}

Progress towards modelling pattern formation in binary blend films was made by the Clarke model of a binary fluid film which coupled phase separation to height variations caused by dewetting [68]. The central part of the model was a free energy functional depending on material volume fraction $\phi(y)$ and film height $h(y)$, given by

$$
\mathcal{F}[\phi(y), h(y)]=\int f(\phi, h)+h g(\phi) d y
$$

where $f(\phi, h)$ is the surface energy, $g(\phi)$ is the bulk free energy, and $y$ is the lateral direction parallel to the film substrate. In the Clarke model, only compositional variations in the lateral directions are possible, so vertical phase separation in the vertical (depth) direction cannot be studied. This also means that a meaningful preferential surface attraction of blend components cannot be included, since the primary way in which preferential surface attraction affects the blend is to cause vertical variation of the composition. Figure 5.3 is a schematic of the Clarke model, which attempts to represent the description of the film by the height $h(y)$ and composition $\phi(y)$ at point $y$ (this schematic is designed to be directly compared with my model, represented in figure 5.4). 


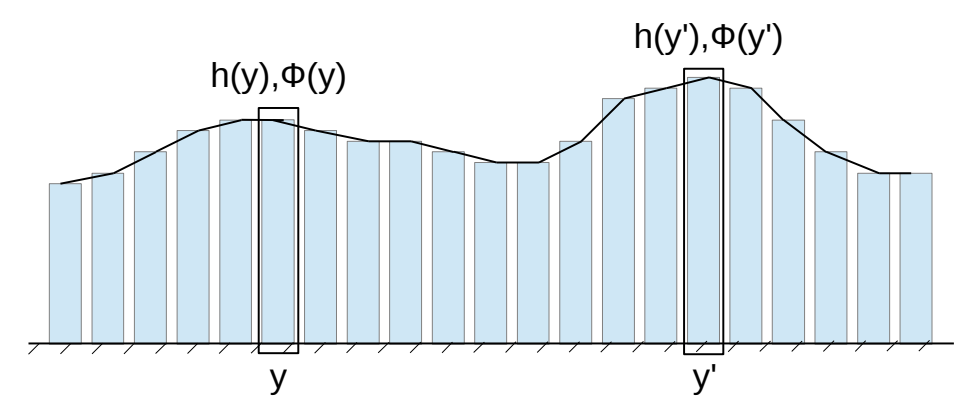

Figure 5.3: 2D schematic of the Clarke Model, representing a fluid layer with a deformable upper surface on a flat substrate. The film height at $y$ is given by $h(y)$, and there is no vertical dependence of the composition $\phi(y)$ at $y . \phi(y)$ could be thought of as the average volume fraction at $y$, but clearly this model would best describe films with no vertical variation of the composition at all.

\section{$5.2 \quad$ A 3D Model}

The model presented in this chapter is essentially an extension of the Clarke model. I introduce a vertical dependence of the composition, and formulate a set of governing equations. The model proceeds via two stages: the lateral movement of material (bulk movement of material that causes roughening); and diffusion (as in the simulations of chapter 4). This model is the most consistent and workable of all of the possible formulations that I investigated, and fortunately makes the most intuitive sense out of all of them.

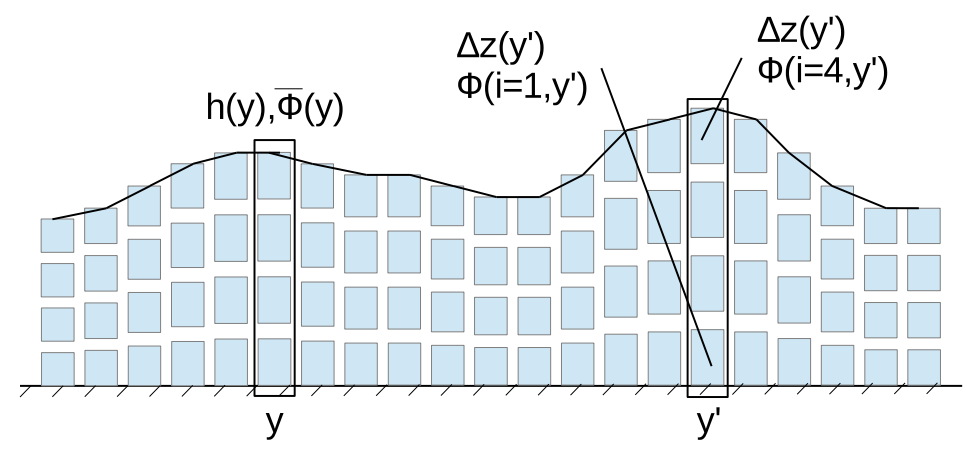

Figure 5.4: 2D schematic of my Model, representing a fluid layer with a deformable upper surface on a flat substrate. Here, the film is divided into $D=4$ grid cells in the vertical direction. The cell-height is given by $\Delta z\left(y^{\prime}\right)=h\left(y^{\prime}\right) / D$, so that the cell-height at $y^{\prime}$ is constant. Each cell has volume fraction $\phi\left(i, y^{\prime}\right)$, where the index $i$ runs from 1 to $D$. The average volume fraction $\bar{\phi}(y)$ is an average over $i$ at point $y$. 


\subsubsection{Vertical dependence of composition}

I first introduce a vertical dependence $z$ into the free energy functional used by Clarke, which I a priori discretise such that a vertical coordinate $z$ is replaced by index $i$ :

$$
\mathcal{F}[\phi(i, y), h(y)]=\int f(\phi, h)+\sum_{i=1}^{D} \Delta z(y) g(\phi) d y,
$$

so that $h(y)=D \Delta z(y)$ i.e. the height at point $y$ is divided into $D$ grid cells of equal height $(\Delta z(y)$ does not depend on $i$ : at any point $y$ all $\Delta z$ values are the same). Figure 5.4 is a schematic of my model, explaining the average volume fraction $\bar{\phi}(y)=D^{-1} \sum_{i=1}^{D} \phi(i, y)$ at point $y$ and the volume fraction $\phi(i, y)$ in individual grid cells (in the schematic 5.4, I have discretised the $y$ direction too, although at this point it is still continuous; it will be discretised for simulations). In order to simplify the notation in the model, it is useful to separate the free energy $\mathcal{F}$ into the following parts

$$
\mathcal{F}[\phi(i, y), h(y)]=F^{\phi}[\phi(i, y)]+F^{h}[h(y)]+F^{\phi, h}[\phi(i, y), h(y)],
$$

where

$$
\begin{aligned}
& F^{\phi}=\int f^{\phi}(\phi)+\sum_{i=1}^{D} \Delta z(y) g(\phi) d y \\
& F^{h}=\int f^{h}(h) d y, \quad F^{\phi, h}=\int f^{\phi, h}(\phi, h) d y .
\end{aligned}
$$

and where I have separated the surface energy into

$$
f(\phi, h)=f^{\phi}(\phi)+f^{h}(h)+f^{\phi, h}(\phi, h),
$$

where $f^{h}(h)$ and $f^{\phi}(\phi)$ depend only on $h$ and $\phi$ respectively, and $f^{\phi, h}(\phi, h)$ contains any cross terms. This separation of the energy into parts simply makes it easier to write a compact form of the model, the convenience mainly being in Eq. (5.4) since this is the only functional containing the sum.

\subsubsection{Two-stage dynamic model}

The dynamic model proceeds via two stages: a height evolution step and a diffusion step. For the height evolution, which involves lateral movement of material, I use the following coupled equations [68]

$$
\begin{aligned}
\frac{\partial h(y)}{\partial t} & =\nabla_{y} \cdot\left(\frac{h^{3}}{3 \eta} \nabla^{*} \mu_{c}(y)\right), \\
\frac{\partial \bar{\phi}(y) h(y)}{\partial t} & =\nabla_{y} \cdot\left(\frac{h^{3} \bar{\phi}}{3 \eta} \nabla^{*} \mu_{c}(y)\right),
\end{aligned}
$$

which is very similar to the formulation of the Clarke model [68], but with a redefined chemical potential gradient $\nabla^{*} \mu_{c}(y)$ which is explained in section 5.2.3. 
This step in the model acts to move material laterally in the film, in such a way that $h(y)$ and $\bar{\phi}(y)$ change. For diffusion, I use the same form as in chapter 4 but generalised to account for a non-uniform grid

$$
\frac{\partial \phi(i, y) \Delta z(y)}{\partial t}=M \nabla \cdot\left[\Delta z \nabla \mu_{\phi \Delta z}(i, y)\right]
$$

where $M$ is the mobility, assumed to be constant.

Equation 5.8 is responsible for the change in the average volume fraction $\bar{\phi}(y)$ at a point $y$. The volume fraction in each grid cell $i$ at $y$ must be changed by the same amount to account for this change in $\bar{\phi}(y)$, so we can think of this step as 'not knowing' that there is any vertical dependence of the composition at all and that the model is essentially the schematic of figure 5.3. This is better explained by the implementation of the forward difference time-step for this stage of the simulation, so I refer the reader to section C.1 in the appendix on the numerical implementation. (Note that the chemical potential gradient $\nabla^{*} \mu_{c}(y)$ does actually account for the vertical dependence of composition in order to make the model physical. This is explained in section 5.2.3).

The diffusive step allows an exchange of material between different individual cells. We will see from the results that diffusion rapidly acts to distribute material vertically, such that once initial vertical phase separation into a transient wetting layer has occured, vertical phase separation is effectively never catching up with lateral phase separation: at any point $y$, the film is as vertically separated as it can be without requiring lateral phase separation such that the overall volume fraction at $y$ changes. This was true in the results of chapter 4 too, in which lateral phase separation takes much longer than vertical phase separation, and it was precisely the growth of lateral inhomogeneities that caused vertical changes in the profile and allowed the bilayer interface to become distorted. This helps justify the form of the two-stage model, in particular the movement of lateral material in the first step which uniformly distributes this material across the grid cells $i$ at point $y$ : even if this even distribution of material is not as physical as if individual transfer between different cells $i$ were allowed at this stage (which would severely complicate the model and would require variation of $\Delta z$ with $i$ ), diffusion rapidly redistributes material appropriately. 


\subsubsection{Definition of the chemical potentials}

I have defined the following

$$
\begin{aligned}
& \nabla^{*} \mu_{c}(y) \equiv \nabla_{y} \mu_{h}(y)+\bar{\phi} \frac{1}{D} \sum_{i=1}^{D} \nabla_{y} \mu_{\phi \Delta z}(i, y), \\
& \mu_{h}(y) \equiv \frac{\delta F^{h}}{\delta h}+\frac{\delta F^{\phi, h}}{\delta h}+\frac{1}{D} \frac{\delta F^{\phi}}{\delta \Delta z}, \\
& \mu_{\phi \Delta z}(i, y) \equiv \frac{\delta F^{\phi}}{\delta(\phi \Delta z)}+\frac{\delta F^{\phi, h}}{\delta(\phi \Delta z)}
\end{aligned}
$$

for the gradients of the chemical potentials in equations (5.7), (5.8) and (5.9).

The diffusion step equations (5.9) together with (5.12) is really a generalisation of the diffusion equation that appeared in chapter 4 for which $\Delta z$ did not vary (in appendix B we see that the chemical potential $\mu_{\phi \Delta z}$ derived from equation (5.2) will actual give the same result as $\delta \mathcal{F} / \delta \phi$ gave in chapter 4 , if $\Delta z$ is constant everywhere). The form of the other equations is explained below.

Equation (5.11) can be derived as follows. It is simple to write

$$
\begin{aligned}
\mu_{h}(y) \equiv \frac{\delta \mathcal{F}}{\delta h} & =\frac{\delta}{\delta h}\left[F^{\phi}+F^{h}+F^{\phi, h}\right] \\
& =\frac{\delta F^{\phi}}{\delta h}+\frac{\delta F^{h}}{\delta h}+\frac{\delta F^{\phi, h}}{\delta h},
\end{aligned}
$$

but the discretisation of $F^{\phi}$ means that $h$ no longer seems to appear in $F^{\phi}$ (if I had written Clarke's Model down in the same formulation as we have here, we would have found $h g(\phi)$ in $F^{\phi}$, and of course I could have written $h / D$ in place of $\Delta z$ in $F^{\phi}$ ). This artefact can be removed by observing that we can write

$$
\begin{aligned}
F^{\phi} & =\int f^{\phi}(\phi)+\sum_{i=1}^{D} \Delta z(y) g(\phi) d y \\
& =\int f^{\phi}(\phi) d y+\sum_{i=1}^{D} \int \Delta z(y) g(\phi) d y,
\end{aligned}
$$

since we can swap the summation and the integration and leave the terms the same. If we now take the functional derivative with respect to $h$ :

$$
\begin{aligned}
\frac{\delta F^{\phi}}{\delta h} & =\frac{\delta}{\delta h} \sum_{i=1}^{D} \int \Delta z(y) g(\phi) d y \\
& =\frac{\delta}{\delta h} \sum_{i=1}^{D} F_{i}^{\phi}=\frac{\delta}{\delta h}\left[F_{1}^{\phi}+F_{2}^{\phi}+\ldots F_{D}^{\phi}\right] \\
& =\frac{1}{D}\left[\frac{\delta F_{1}^{\phi}}{\delta \Delta z}+\frac{\delta F_{2}^{\phi}}{\delta \Delta z}+\ldots \frac{\delta F_{D}^{\phi}}{\delta \Delta z}\right] \\
& =\frac{1}{D} \sum_{i=1}^{D} \frac{\delta F^{\phi}}{\delta \Delta z}
\end{aligned}
$$


where I have used the fact that the functional derivative commutes on the second line, and on the third line I have used the fact that $h=D \Delta z(\Delta z$ does not depend on $i$ ). Really, $\Delta z$ is defined as $\Delta z=h / D$, so in a way $h$ does appear in $F^{\phi}$. So the functional derivative with respect to height at point $y$ can be written as an average over $i$ of the functional derivatives with respect to cell height for each grid cell at point $y$. The latter step is very important, as it is one of the important insights that allowed a vertical dependence to be included in my model. Without the a priori discretisation, how could we write an expression for $\delta F^{\phi} / \delta h$ with $F[\phi(z)]=\int_{0}^{h} \ldots f(\phi) \ldots d z$ ? That equation (5.16) exactly reduces to that for the Clarke Model (for a vertically homogeneous film) when the values of $\phi_{i}(z)$ are all the same gives us confidence that it is correct.

\section{The combined gradient of the chemical potential}

Concerning the gradient of the chemical potential $\nabla^{*} \mu_{c}$ in equations (5.7), (5.8) and (5.10), we can see that without variation in the $z$ direction, and therefore no variation in $i$, that $(5.10)$ reduces down to

$$
\nabla^{*} \mu_{c}(y) \equiv \nabla_{y} \mu_{h}(y)+\phi \nabla_{y} \mu_{\phi \Delta z}(i, y)
$$

in accordance with Clarke's model. In the latter equation, we have $\phi \equiv \bar{\phi}$, since the model assumes the we can describe the film at a point $y$ by the average composition at that point. Putting the $i$ dependence back in, and writing $\phi \rightarrow \bar{\phi}$ in accordance with the role of $\phi$ in Clarke's model, we can write the latter equation in the form:

$$
\phi \nabla_{y} \mu_{\phi \Delta z}(i, y) \rightarrow \bar{\phi} \frac{1}{D} \sum_{i=1}^{D} \nabla_{y} \mu_{\phi \Delta z}(i, y),
$$

where I have averaged the lateral gradients of the chemical potential $\mu_{\phi \Delta z}$ in each individual grid cell at $y$, in order to better account for the 'interface' between the vertical 'columns' at point $y$. It is via this averaging that the $i$ dependence is removed. Note that I could have also done $\bar{\phi} \frac{1}{D} \nabla_{y} \sum_{i=1}^{D} \mu_{\phi \Delta z}(i, y)$ i.e. taken the gradient of the average chemical potential, but this would not have properly taken account of the interface between two side-by-side 'columns' of fluid at point $y$, which would have been less physical since I would like to properly account for the vertical variation in the film profile. Also, I settled on using $\bar{\phi}$ outside of the averaging, rather than using $\phi$ within the averaging, not because it appeared to make any difference at all, but because this is more in line with the original formulation of Clarke, and keeps the symmetry in equations (5.7) and (5.8).

At this point the model is completely generalised, and satisfies the thermodynamic stability criterion required for an initially homogeneous film [77, 68], since the model reduces down to Clarke's model in the case of no vertical variations.

\section{A note on the functional derivatives}

Note the presence of functional derivatives of Eq. (5.2), using the notations and definitions in Eqs. (5.3)-(5.5). We must take care in calculating the functional 
derivatives in Eqs. $5.10-5.12$ by writing $(\phi \Delta z) / \Delta z$ in place of $\phi$ when performing derivatives [84]. This is done in the appendix B. This makes sure that the movement of material is with regards to gradients in the proper quantities: $\phi$ is not a conserved variable in this system of equations, $h$ and $\phi \Delta z$ are conserved, and must be to satisfy material conservation (in Chapter $4, \phi$ was conserved because film depth was fixed).

\subsection{Application to polymer-blend thin films}

To illustrate the model, I apply it to a binary polymer blend of components $A$ and $B$ between selectively attracting walls. The bulk free energy is given by

$$
g(\phi)=f_{F H}(\phi)+\kappa(\phi)(\nabla \phi)^{2},
$$

where $f_{F H}(\phi)=(1 / N)[\phi \ln (\phi)+(1-\phi) \ln (1-\phi)]+\chi \phi(1-\phi)$ and $\kappa(\phi)=$ $a^{2} / \phi(1-\phi)$, where $a$ is the underlying Flory-Huggins lattice spacing, $N$ is the degree of polymerization (same for both $A$ and $B$ ) and $\chi$ is the Flory-Huggins interaction parameter. The $\phi$-dependant surface energy is given by

$$
f^{\phi}(\phi)=f_{1}(\phi) \delta_{i 1}+f_{D}(\phi) \delta_{i D},
$$

where $f_{S}=h_{S} \phi+(1 / 2) g_{S} \phi^{2}$ and $h_{S}$ and $g_{S}$ are phenomenological parameters. $S=1, D$ and $\delta_{i S}$ is the Kronecker delta function, so the surface energies only act in the cells adjacent to the film surfaces. For the height dependant surface energy I used [68]

$$
f^{h}(h)=\sigma\left(\nabla_{y} h(y)\right)^{2}+\epsilon / h^{8}
$$

where the first term accounts for energy costs of curvature in the film surface and the second term prevents complete dewetting of the substrate (which, although observed, is to some extent is a numerical convenience). Equation (5.21) implies that the Hammaker constant is zero; the results do not depend on the top and lower surfaces 'feeling' each other. Note that I have tried to keep the specific terms in the model extremely minimal, which should allow for general principles to be discovered through the simulations.

\section{Specific Parameters}

I performed the simulations in $3 \mathrm{D}$, so that the lateral dimension comprises of $x$ and $y$ directions. I scaled space by $z^{\prime}=\left|\chi-\chi_{S}\right|^{1 / 2} z / a$ (applied to $x$ and $y$ too) and time by $\tau=N M\left|\chi-\chi_{S}\right|^{2} t / a^{2}$, where $\chi_{S}$ is the value of $\chi$ on the spinodal curve. I used the following parameters: $N=100, \bar{\phi}=0.5$ (unless specified), $a=1, \eta=1000, M=1, \epsilon=0.010, \sigma=0.01$ (unless specified). I used a square simulation grid in the lateral plane, with $\Delta x^{\prime}=\Delta y^{\prime}=0.173$, and have used $D=16$ grid cells to discretise the vertical direction. For the results presented here, I used $\chi=0.050$ (this large value results from $a=1$, but $a$ and $\chi$ are both absorbed into the scaling; this allows the the films to be relatively thin, and so less area of film is need for the simulations). Random thermal currents $J^{T}$ from 
a Gaussian distribution of width 0.01 were applied in the diffusion stage of my simulations, providing an additional term $+\nabla \cdot\left(\Delta z J^{T}\right)$ to Eq. (5.9) [65], which is discussed in appendix $\mathrm{C}$.

I used the following surface parameters and terminology, in common with chapter 4: for 'antisymmetric' films $h_{1}=-0.05, g_{1}=0.18, h_{D}=-0.13, g_{D}=$ 0.18 (a B-attracting substrate and an A-attracting surface, such that the walls attract opposite components in exactly the same way); for 'asymmetric' films $h_{1}=-0.05, g_{1}=0.18, h_{D}=g_{D}=0$ (a B-attracting substrate and a neutral surface); and for 'symmetric' films $h_{1}=h_{D}=-0.05, g_{1}=g_{D}=0.18$ (both the substrate and the surface are B-attracting).

\section{Functional Derivatives}

The expressions for the functional derivatives required in the model, the derivations of which can be found in the appendix B, are given by

$$
\begin{aligned}
\frac{\delta F^{\phi}}{\delta(\phi \Delta z)}= & +\partial_{\phi} f_{F H}(\phi)-\left(\partial_{\phi} \kappa\right)(\nabla \phi)^{2}-2 \kappa(\phi) \nabla^{2} \phi \\
& +(\Delta z)^{-1}\left[+2 \kappa(\phi) \nabla_{z} \phi+\partial_{\phi} f_{h}\right] \delta_{h}^{z} \\
& +(\Delta z)^{-1}\left[-2 \kappa(\phi) \nabla_{z} \phi+\partial_{\phi} f_{0}\right] \delta_{0}^{z} \\
& -\frac{2}{\Delta z} \kappa(\phi) \nabla(\Delta z) \cdot \nabla \phi, \\
\frac{\delta F^{\phi}}{\delta \Delta z}= & +f_{F H}(\phi)+\kappa(\phi)(\nabla \phi)^{2} \\
& -\phi \partial_{\phi} f_{F H}(\phi)+\phi\left(\partial_{\phi} \kappa\right)\left(\nabla_{y} \phi\right)^{2}+2 \phi \kappa(\phi) \nabla_{y}^{2} \phi \\
& -4 \kappa(\phi)\left(\nabla_{z} \phi\right)^{2}-\phi\left(\partial_{\phi} \kappa\right)\left(\nabla_{z} \phi\right)^{2} \\
& -(\Delta z)^{-1}\left[+2 \phi \kappa(\phi) \nabla_{z} \phi+\phi \partial_{\phi} f_{h}\right] \delta_{h}^{z} \\
& -(\Delta z)^{-1}\left[-2 \phi \kappa(\phi) \nabla_{z} \phi+\phi \partial_{\phi} f_{0}\right] \delta_{0}^{z} \\
& +\frac{2 \phi}{\Delta z} \kappa(\phi) \nabla(\Delta z) \cdot \nabla \phi, \\
\frac{\delta F^{h}}{\delta h}= & -\frac{8 \epsilon}{h^{9}}-2 \sigma \nabla^{2} h .
\end{aligned}
$$

Due to the discretisation of the free energy functional (5.2), there are two approaches to calculating the expressions above, which yield very slightly different results. This is explained in the appendix B. Whilst the Model I present here does not depend on which 'version' of the functional derivatives are used, since the model is supposed to solve the general problem of simulating a dewetting film with 3D phase separation, the different approaches may be important in some ways that were not clear in testing the model. 


\subsection{Results and Discussion}

Figs. 5.5 and 5.7 show an antisymmetric and an asymmetric film, respectively, at different stages in evolution. For these 3D plots, time increase from (a) to (d), where (a) vertical phase separation of initially nearly homogeneous film; (b) bilayer film; (c) breakup of the bilayer; (d) laterally segregated film. Figs. 5.6 and 5.8 are $2 \mathrm{D}$ plots showing the surface volume fraction (left-hand side, colour-bar shown in 3D plots) and the film height (right-hand side, colour-bar shown) for the films in Figs. 5.5 and 5.7, respectively. For these 2D plots, time increases from top to bottom, the particular plots having been selected to show the breakup of the bilayer (top), the emergence of lateral phases (middle), and the laterally segregated film (bottom: same time as shown in (d) of the 3D plots figures 5.5 and 5.7). Figure 5.11 shows $2 \mathrm{D}$ plots of the final surface volume fraction and film height of several symmetric films with different initial average volume fractions.

\subsubsection{Antisymmetric Films and Asymmetric Films}

Figs. 5.5 and 5.7 show an antisymmetric and an asymmetric film, respectively, at different stages in evolution. In both cases, the film initially undergoes vertical stratification (a) due to the preferential surface attraction, forming a bilayer (b). This bilayer then breaks up via the surface bifurcation mechanism ([67] and chapter 4) in which the single value of the order parameter at the film surfaces divides as laterally coexisting phases appear (c), and the film becomes laterally segregated (d). Hence the films undergo lateral phase separation via a transient wetting layer (the instability of the bilayer is studied in [81] and [82], although due to non-diffusive transport mechanisms).

When the film is in the bilayer state as in figures 5.5(a) and 5.7(a) there is no significant roughening of the film surface. The surface plots of figures 5.6 and 5.8 show that as the bilayer begins to breakup (top), showing significant phase separation at the top surface, the surface roughens quite significantly. This may correspond to the increased roughness of the surface prior to lateral phase separation of the bilayer, as reported in Ref. [60]. It seems that the onset of phase separation in the lateral direction induces this roughening. As the lateral phases emerge due to the breakup of the bilayer, as in figures 5.6(middle) and 5.8(middle), dewetting couples strongly to the lateral phase separation, causing the surface roughening to shadow the phase separating morphology. Earlier signs of this coupling can also be faintly seen in the top sub-figure of figure 5.8, where the B-rich material has broken the surface.

The laterally segregated morphology that results depends on the surface regime. Figure 5.6 shows that the antisymmetric film laterally phase separates into a bicontinuous morphology of both the A-rich and B-rich phases. The simulations show that the B-rich phase protrudes from the A-attracting film surface. From a purely thermodynamic perspective that considers the lateral phases in 1D (as in chapter 3) it is not clear why either phase would be higher than the other [46]. However, the kinetics of the breakup of the bilayer appears to promote the B-rich phase, which previously formed the bottom layer of the bilayer. This may be 

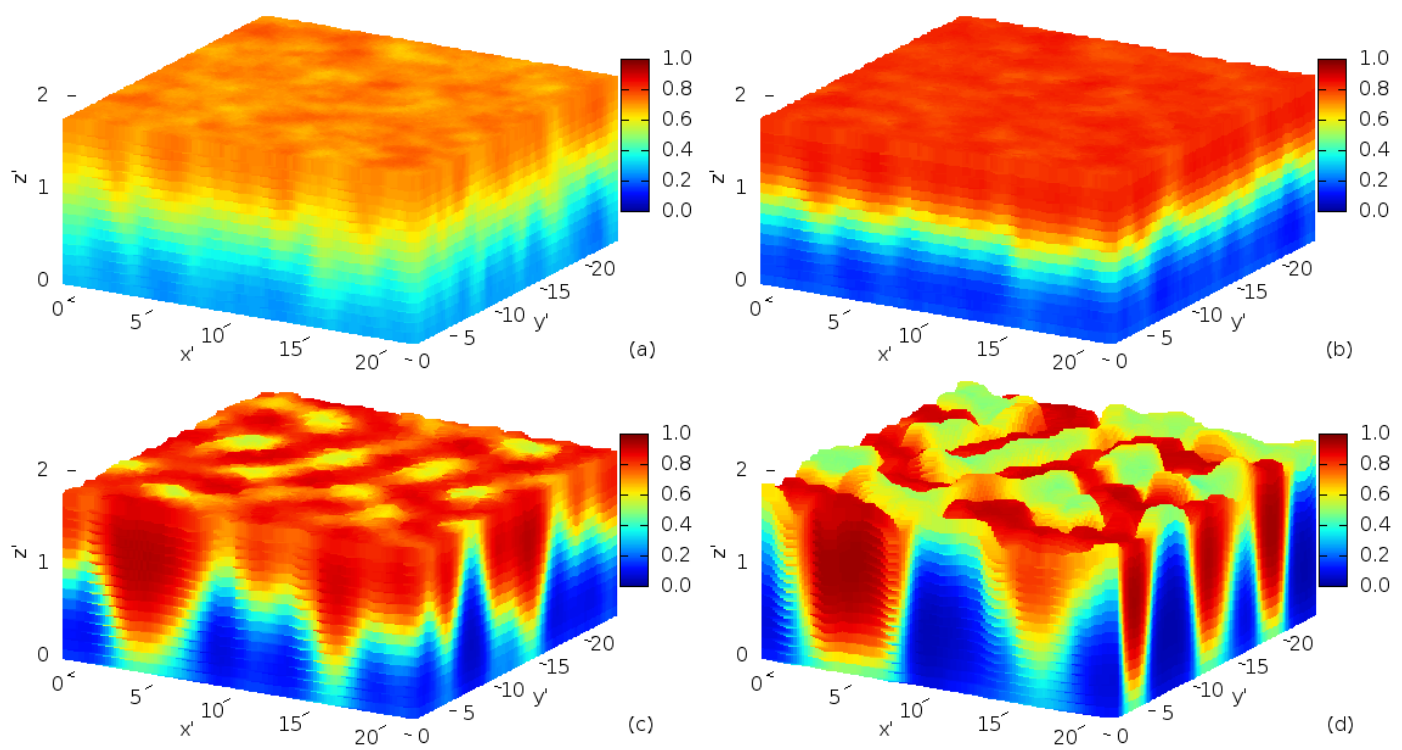

Figure 5.5: Antisymmetric film of average height $h^{\prime}=1.73$. (a) initial vertical phase separation $\tau=25$; (b) bilayer film $\tau=100$; (c) breakup of bilayer $\tau=500$; (d) laterally segregated film $\tau=1263$. The trapezoidal shape of the lateral phases can be clearly seen, meaning that the $B$-rich phase makes less contact with the top surface than the $A$-rich phase. This may be responsible for the kinetics that cause the $B$-rich phase to become higher than the $A$-rich phase, despite the symmetry of the system.

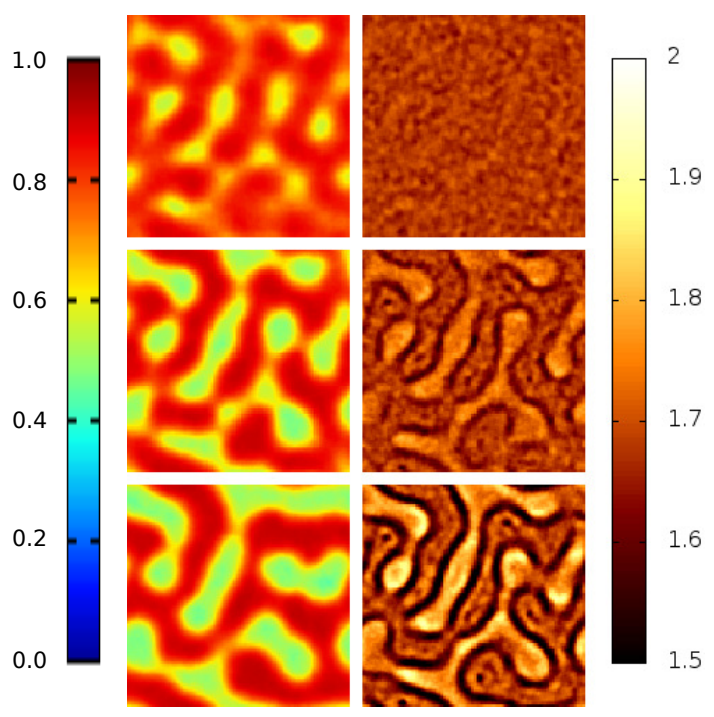

Figure 5.6: Surface view for film shown in Fig. 5.5 (left: volume fraction, as in Fig. 5.5; right: film height, colour-bar shown). Top: breakup of the bilayer $(\tau=500)$; Middle: appearance of lateral phases $(\tau=830)$; Bottom: further roughening as lateral phases coarsen $(\tau=1263)$. The surface morphology is bicontinuous, with the B-rich phase protruding higher than the A-rich phase. 

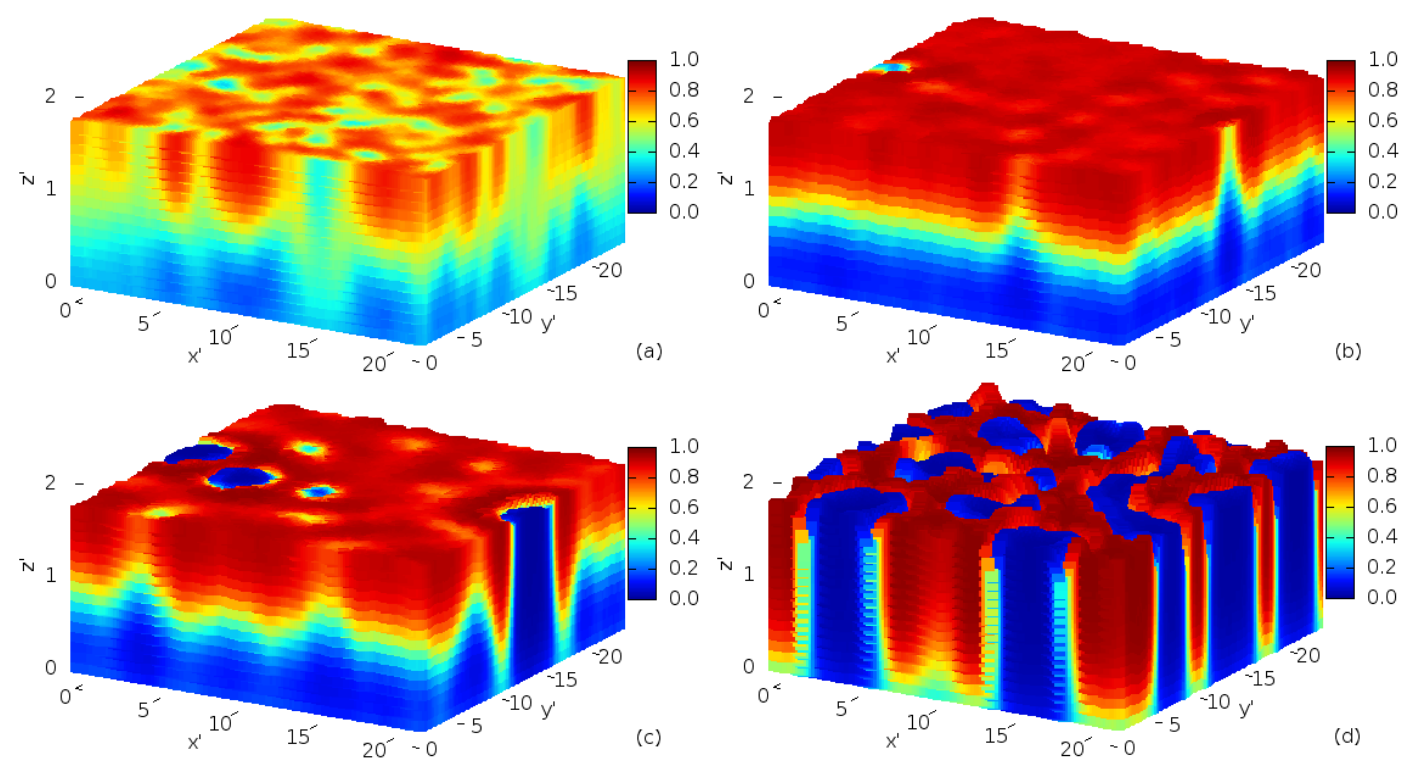

Figure 5.7: Asymmetric film of average height $h^{\prime}=1.73$. (a) initial vertical phase separation $\tau=40$; (b) bilayer film $\tau=160$; (c) breakup of bilayer $\tau=540$; (d) laterally segregated film $\tau=1200$. The B-rich phase breaks the surface even though the film is vertically stratified (b), so that it appears that there is a template of the laterally segregated morphology already present at this point, which determines where the breakup of the bilayer occurs due to surface bifurcation, as in (c).

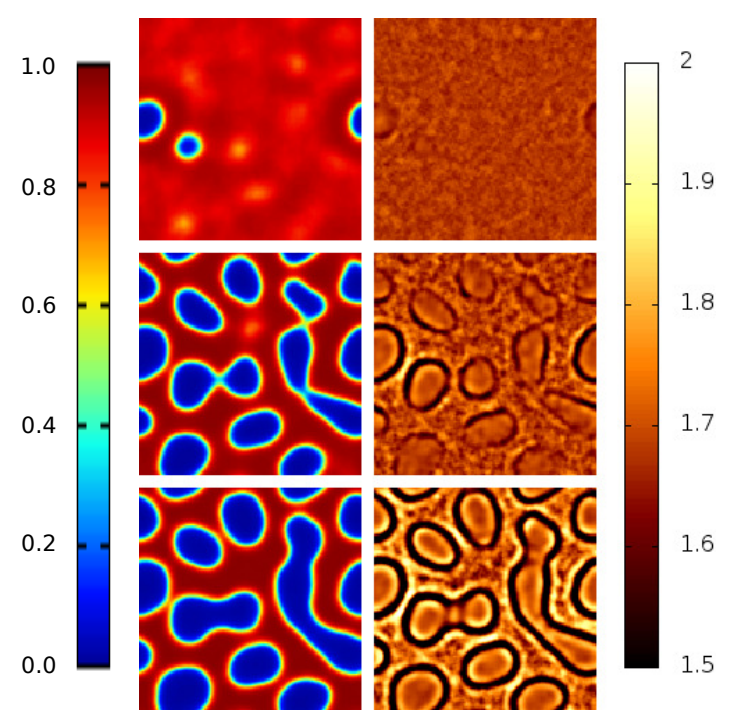

Figure 5.8: Surface view for film shown in Fig. 5.7 (left: volume fraction, as in Fig. 5.7; right: film height, colour-bar shown). Top: beginning of breakup of the bilayer, $(\tau=400)$; Middle: more lateral phases break the surface, destroying the bilayer $(\tau=840)$; Bottom: further roughening as lateral phases coarsen $(\tau=1200)$. The A-rich phase is continuous, encapsulating islands of the B-rich phase. 
because of the trapezoidal shape of the laterally coexisting phases, which means that in this case it is the $B$-rich phase that makes less contact with the top surface than the $A$-rich, and since dewetting appears to primarily proceed at the interface between the laterally coexisting phases, the $B$-rich phase will become higher than the $A$-rich phase, since it is narrower at the point where it meets the surface and thus requires less material to be transferred into it for the same height increase. The laterally segregated state of the asymmetric film shown in figure 5.8 is a continuous A-rich phase which encapsulates islands of the B-rich phase. This is caused by depletion of B-component in the film due to the B-attracting substrate, leading to an excess of A-component in the rest of the film.

In the case of the asymmetric film of figure 5.7, an important point can be made about the bilayer state (a), namely that the film is definitely vertically stratified into a bilayer even though it is clear that the surface of the film would suggest that the film is laterally segregated (the snapshot of the bilayer has been chosen to show this). The reason for this is that lateral phase separation is proceeding from the top surface in this case [50], as in chapter 4. The variations that can be seen in the bilayer correspond to where the lateral phases are in the laterally segregated film. In Ref. [85], the authors observe that a bicontinuous morphology is maintained throughout the spin-casting process, and conclude that this means no bilayer initially formed. Their conclusions therefore contradict earlier results reporting that a bilayer did initially form [60]. My results bring these two contradicting interpretations together, showing that phase separation observed at the surface does not indicate that there is no vertical stratification.

\section{Quantitative Analysis}

Figures 5.9 and 5.10 are plots, against (scaled) time $\tau$, of the free energy (calculated with equation (5.2)) per unit area, the standard deviation (S.D.) of the (scaled) height $<\left(h^{\prime}(y)-h_{0}^{\prime}\right)^{2}>^{\frac{1}{2}}$, and the S.D. of the average volume fraction $<\left(\bar{\phi}(y)-\bar{\phi}_{0}\right)^{2}>^{\frac{1}{2}}$, for an antisymmetric film and an asymmetric film respectively (with the same parameters as figures 5.5 and 5.7, respectively). $<\left(h^{\prime}(y)-h_{0}^{\prime}\right)^{2}>^{\frac{1}{2}}$ gives some measure of the surface roughness of the film, and $<\left(\bar{\phi}(y)-\bar{\phi}_{0}\right)^{2}>^{\frac{1}{2}}$ gives some measure of the degree of lateral segregation of the film (although it should be noted that the variable $\bar{\phi}$ is not a conserved quantity). The results for the antisymmetric and asymmetric film are qualitatively the same in every regard, and so I will only explicitly discuss figure 5.9 for the antisymmetric film.

Early on $\tau<100$, the free energy falls rapidly as the film initially vertically phase separates into a bilayer (transient wetting layer), at which point the free energy plateaus between $\tau=100$ and $\tau=300$ since the film is in metastable equilibrium. Around $\tau=300$, lateral inhomogeneities have grown enough that the bilayer begins to breakup (surface bifurcation) and the free energy falls away from the plateau. At this same time, the S.D. of height changes significantly, levelling off (and even dropping slightly) from the initial linear rise: this initial rise may be the roughening of the bilayer prior to lateral phase separation, as mentioned above and reported in Ref. [60]. A change in the S.D. of $\bar{\phi}(y)$ also happens at exactly this time, the gradient becoming shallower. I suggest that the latter 


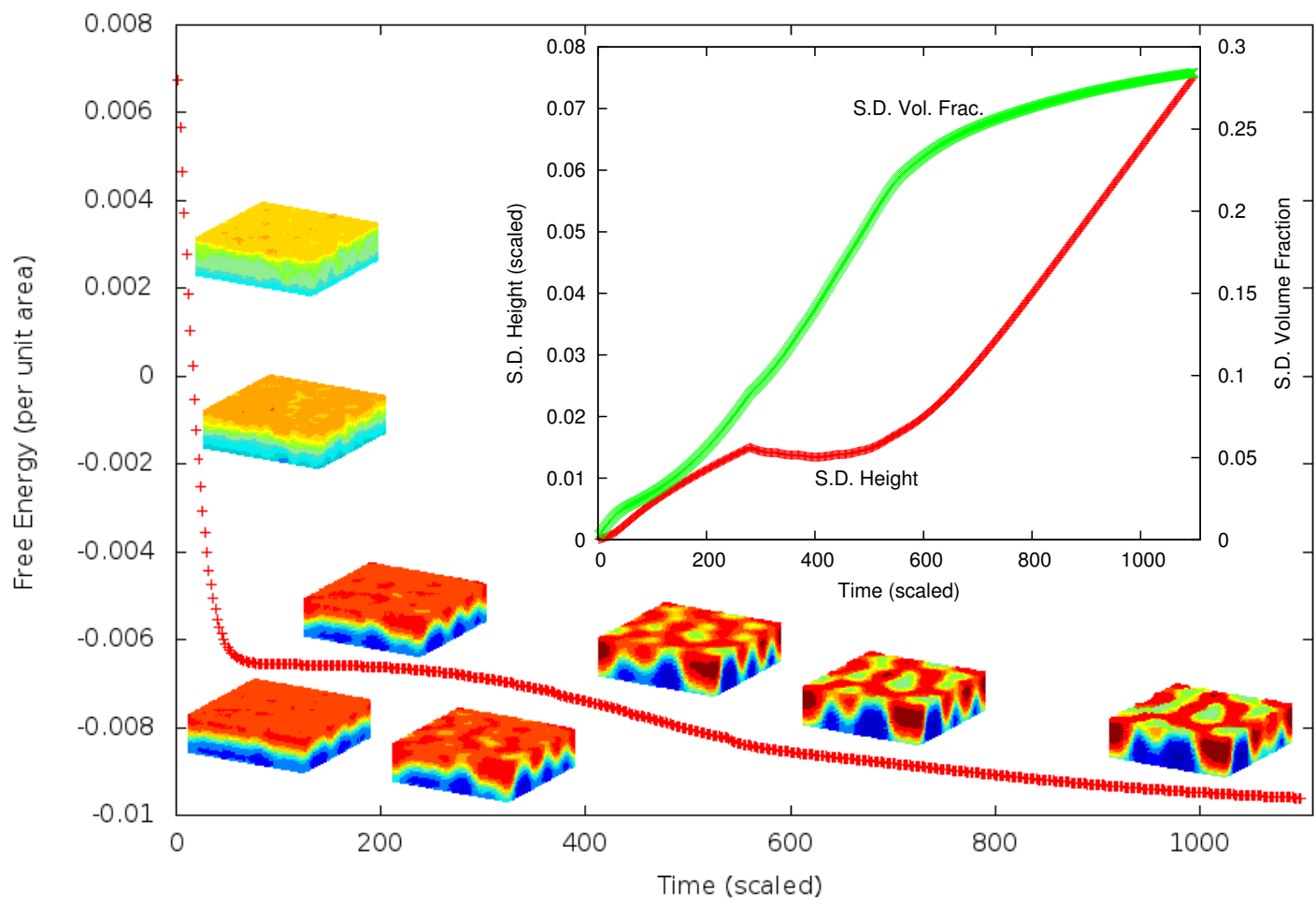

Figure 5.9: Antisymmetric film of average height $h^{\prime}=1.73$ (with the same parameters of figure 5.5 but with $(3 / 4)^{2}$ the area). The standard deviations (S.D.) of height $h^{\prime}(y)$ and average volume fraction $\bar{\phi}(y)$ compliment the features that can be inferred from the $3 \mathrm{D}$ simulation snapshots and free energy curve. The free energy plateaus for the bilayer (TWL), and falls off fairly rapidly as the bilayer breaks up. As the laterally coexisting phases appear from this breakup, the film becomes more structured; the S.D. of height and $\bar{\phi}(y)$ reflect this. Once the laterally coexisting phases have formed, the S.D. of height begins to rapidly increase as surface roughening shadows the underlying phase separated morphology as a means to lower the free energy. At long times when lateral phase separation is mostly complete, the S.D. of $\bar{\phi}(y)$ and the free energy level out, but the surface roughness still increases to further lower the free energy.

observations both correspond to the initial lateral inhomogeneities that breakup the bilayer becoming more structured as laterally coexisting phases appear.

At around $\tau=550$ the laterally coexisting phases have mostly formed and therefore the increase in the S.D. of $\bar{\phi}(y)$ levels off. At this point, the S.D. of height begins to rapidly rise in a linear fashion, now that the surface roughening shadows the underlying phase separated morphology in order to further lower the free energy of the film, as shown in the continuous decrease in the free energy. At $\tau=1000$, the free energy levels out as $<\left(\bar{\phi}(y)-\bar{\phi}_{0}\right)^{2}>^{\frac{1}{2}}$ levels out, since lateral phase separation is mostly complete. The film is still roughening, which further lowers the free energy. It seems to be energetically favourable for dewetting to occur at the interfaces between the laterally coexisting phases, as shown in figures 5.6 and 5.8, as the film appears to be able to lower its free energy in this way. 


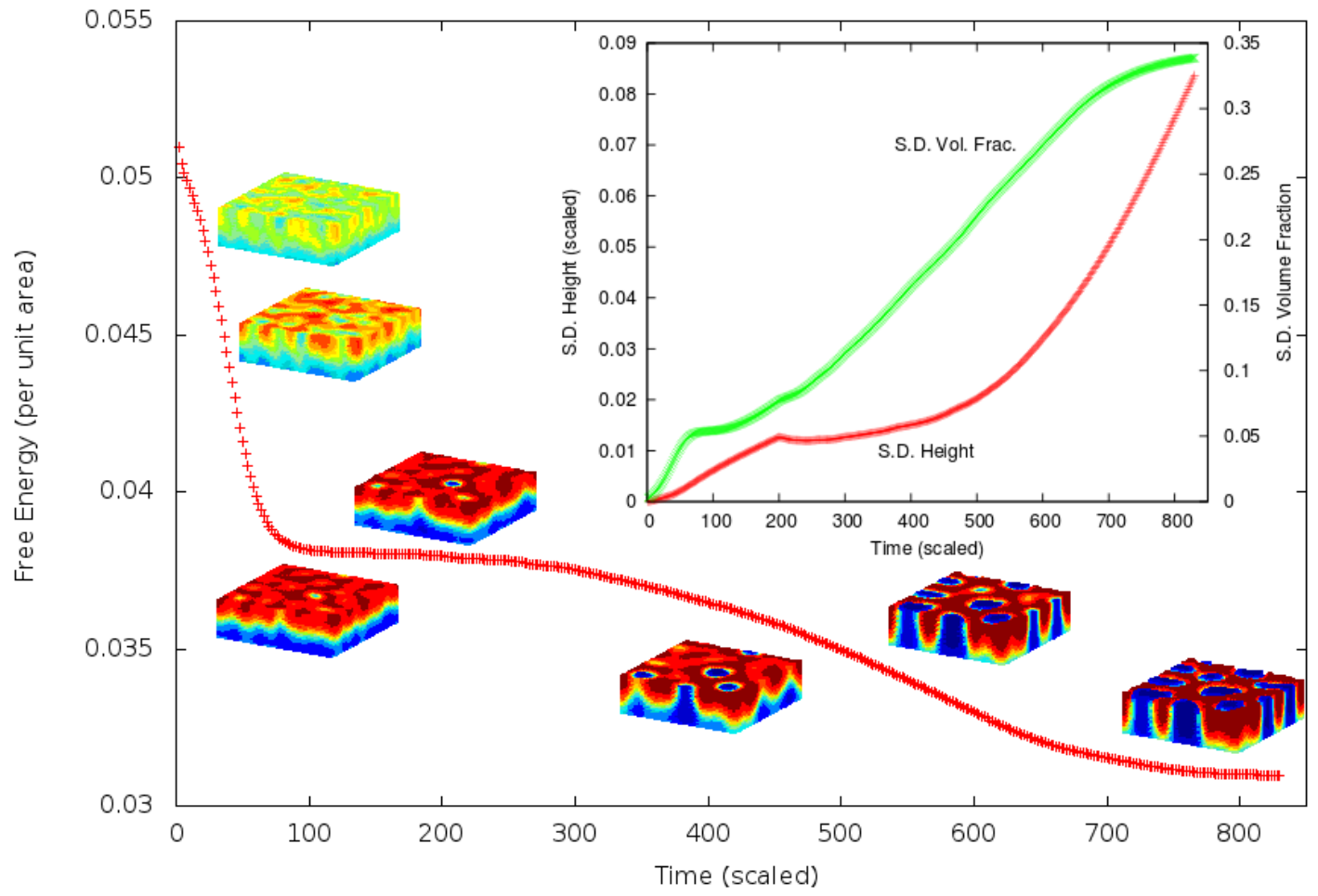

Figure 5.10: Asymmetric film of average height $h^{\prime}=1.73$ (with the same parameters of figure 5.7 but with $(3 / 4)^{2}$ the area). The standard deviations (S.D.) of height $h^{\prime}(y)$ and average volume fraction $\bar{\phi}(y)$ compliment the features that can be inferred from the 3D simulation snapshots and free energy curve. The evolution is essentially qualitatively the same as that described in the caption of figure 5.9.

\section{Validation of the Model}

Equation (5.10) shows that the amount of material that is moved laterally is based on the average volume fraction $\bar{\phi}(y)$ and the average of the lateral gradients of $\mu_{\phi \Delta z}(i, y)$. Material moved in this way is evenly distributed amongst the grid cells at $y$ in order that the average volume fraction at $y$ changes, as explained in Appendix C equation C.15 (this is ultimately because $\Delta z$ does not vary with $i$ ). Equations (5.7) and (5.8), combined with equation (5.10), means that the height evolution step may not, in theory, always lead to a lowering of the free energy, since both the distribution of material across the vertical dimension and the amount of material transferred laterally may not necessarily be optimal. However, the diffusion step rapidly distributes material vertically to compensate for this, and the free energy in figures 5.9 and 5.10 show that the model functions very well. Its not clear if this would be true at very long time, once lateral diffusion has effectively ceased but height evolution still continues (the simulations numerically decouple at longer times as the height differences between simulation grid cells becomes large; much smaller $\Delta y$ would be needed to study longer times). 


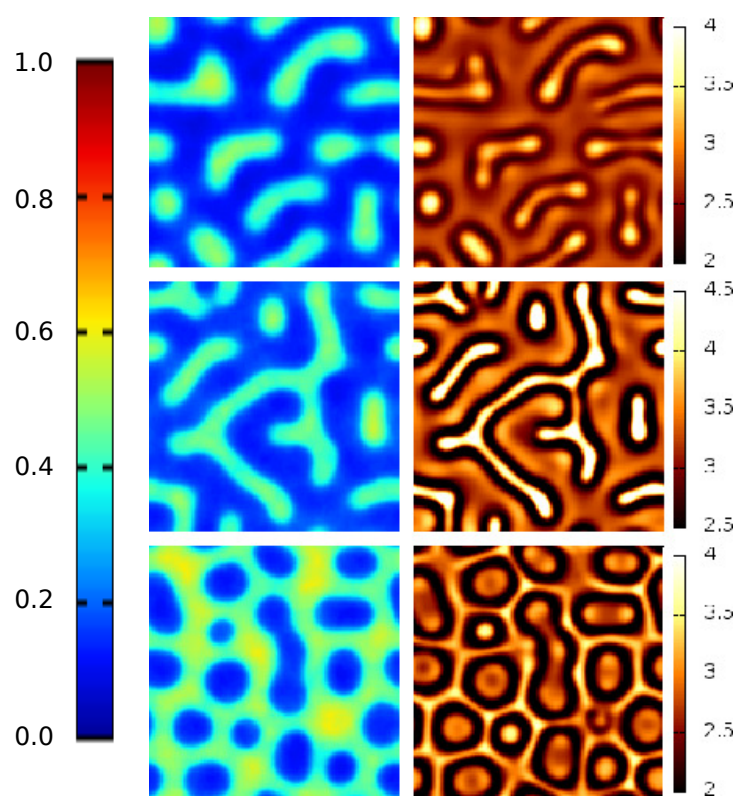

Figure 5.11: Surface view of the laterally segregated states of three Symmetric films, with different $\bar{\phi}$, which initially form a trilayer structure: both surfaces coated by a B-rich layer, with an A-rich layer sandwiched in between (left: volume fraction, as in Figs. 5.5 and 5.7; right: film height, colour-bar shown). The scaling of height depends on $\chi_{S}$, which varies with $\bar{\phi}$ (see sections 1.4 and 2.4). Top: $\left(\sigma=0.03, \bar{\phi}=0.2, h^{\prime}=2.74\right.$ ) droplets of A-rich phase encapsulated by B-rich phase $(\tau=700)$; Middle: $(\sigma=0.01$, $\left.\bar{\phi}=0.3, h^{\prime}=3.24\right)$ droplets joining up as amount of A component increases $(\tau=980)$; Bottom: $\left(\sigma=0.01, \bar{\phi}=0.5, h^{\prime}=2.60\right)$ the A-rich phase forms a protruding matrix encapsulating the lower B-rich phase $(\tau=1200)$.

\subsubsection{Symmetric Films}

I also performed simulations of symmetric films, in which both surfaces preferentially attract the B-component, leaving an A-rich phase sandwiched in the middle of the film i.e. a trilayer structure. I used slightly thicker films for these simulations, since there are three layers in the film and I wanted these layers to be sufficiently distinct. I will briefly discuss the results from these simulations, although in the context of this thesis they are of less interest than results for the lateral phase separation of a bilayer film. I have only shown, in figure 5.11, the surface profiles of the final laterally segregated state, for various blend ratios (values of $\bar{\phi}$ ). For the symmetric blend $\bar{\phi}=0.5$ (top), the final roughened morphology highly resembles that found in experiments, when the encapsulating phase protrudes from the film $[70,72]$. For blends with a lower ratio of A-material $\bar{\phi}=0.3$ (middle), such that the non-wetting A-component is the minority component, the A-rich lateral phase becomes encapsulated in the B-rich phase, with the A-rich phase protruding but no longer continuous. For $\bar{\phi}=0.2$ (bottom), droplets of the minority phase form, which could, if other hydrodynamic flow mechanisms like those suggested in Refs. [69, 71] were present, allow the formation of pancake-like droplets of the minority phase. 


\subsection{Summary}

I have presented results from a new model for thin films of multicomponent fluids, applied to binary polymer blend thin films. The results show that roughening couples to phase separation, resulting in stages of surface roughening corresponding to distinct stages of phase separation. The results can be interpreted in terms of the phase equilibria of polymer films [50,67]: the transient wetting layer that forms before the film laterally phase separates is a metastable state, the phase equilibria of the laterally segregated state corresponding to the equilibria of global equilibrium. In the transient wetting layer, there does not seem to be a route to lower the free energy of the film by roughening, but the roughening begins with the onset of lateral phase separation, as a means to lower the free energy of the film as lateral inhomogeneities grow.

Perhaps as important as the general results concerning coupled surface roughening and lateral phase separation in polymer-blend thin films is the formulation of the model so as to allow a vertical dependence of the composition, which has never been done before in any general way. I hope that the idea behind this model can be utilised by others to formulate similar 3D models. 



\section{Summary of Research and Outlook}

In Chapter 3, I extended a Hamiltonian Phase Portrait method for visualising and calculating equilibrium profiles of polymer-blend thin films, allowing the method to be applicable to the general case of film asymmetry, though I focussed on a symmetric blend between an attracting surface and a neutral surface. I explained how a Lagrange multiplier distorts the phase portraits away from symmetry, and how the location of fixed points can be used to explain the behaviour of equilibria as film depth and temperature regimes are changed. I also discussed laterally coexisting solutions, necessarily limiting to the case in which the phase portraits are not distorted since the $1 \mathrm{D}$ phase portraits are not able to take account of the lateral interface between phases. By considering the role of the Lagrange multiplier, I argued from a thermodynamic perspective that the coexisting phases could have different depths in order that the free energy of the film is reduced, possibly leading to a laterally segregated film with a roughened surface.

In Chapter 4, I studied lateral phase separation via a transient wetting layer using simulations and Hamiltonian Phase Portraits and introduced a Surface Bifurcation mechanism, involving effective boundary conditions at the film surfaces, explaining the particular dynamics of the breakup of the transient wetting layer. I derived a diffusion equation suitable for studying polymer-blend films, in which I used a first-principles derivation to derive the proper forms of the surface terms. I compared the results from simulations to the equilibria calculated from the Hamiltonian phase portrait method to demonstrate that the diffusion equations were accurate and precise, and showed that the 1D calculated equilibria actually appear in the simulations. This means that the lateral interfaces between coexisting phases do not prevent $1 \mathrm{D}$ considerations of equilibria from being useful, at least in terms of the states which films evolve through and into. I showed that films evolve first towards a metastable bilayer configuration, determined by the lowest energy independently-existing equilibria of the films, and then evolve towards laterally coexisting phases corresponding to global equilibrium. I explained the dynamics of the breakup of the transient wetting layers for a variety of wallblend interaction regimes, explaining how the surface bifurcation mechanism may explain many general results from spin-casting experiments.

In Chapter 5, I formulated a 3D model of coupled phase separation and surface roughening by solving the problem of introducing a vertical dependence of the film composition into a model of a dewetting film. I applied the model to a polymer-blend thin film and used it to investigate surface roughening for films with different surface-blend interaction regimes. I showed that lateral phase separation via a transient wetting layer has distinct stages of surface roughening, resulting in pattern formation whereby the surface roughening shadows the underlying phase separated morphology. Both the kinetics of dewetting and the shapes of the underlying equilibria determine the pattern formation.

The work in this thesis has hopefully revealed some very general fundamental aspects of phase separation in polymer-blend thin films. I have implicitly focussed on investigating what is necessary and sufficient to explain phenom- 
ena in film evolution; how stages of film evolution can be explained by phase equilibria i.e. a thermodynamic perspective. The work is based on a mean-field description of a polymer-blend, and so cannot necessarily be relied upon to give quantitative results. Fortunately, mean-field descriptions of polymer blends are known to generally give good results, and polymer blends are identified throughout the literature as ideal systems in which to study phase transitions and related phenomena.

It would seem that this work could equally apply to simple fluid blends. This is true in as much as the equations can be applied to either a polymer blend or a simple fluid blend, provided an appropriate choice for the coefficient of the gradient energy. However, mean-field descriptions of simple fluids do not work very well in practice, for a similar reason that they don't work well for dilute polymer solutions. In the latter case, enthalpic interactions cause solute to clump together, leading to regions of (nearly) pure solvent and (nearly) pure solute. A mean-field description of the solution, derived on the basis that the probability of lattice sites being occupied by polymer segments (solvent molecules) is given by the local polymer (solvent) volume fraction, would fail to describe such a dilute solution. The case is not dissimilar for simple fluid blends. Mean-field theories don't account for local fluctuations in the mean field, and well defined features like the spinodal curve can become artefacts of the theory that don't necessarily make useful predictions. In dense polymer blends with a high molecular weight, the interconnectivity of chains means that fluctuations are less important and mean-field descriptions generally work well.

Flory-Huggins-de Gennes theory (the mean-field theory used throughout this thesis) allows a polymer-blend to be identified by the interaction parameter $\chi$, which can be experimentally determined. As per the scaling in chapters 4 and 5, the length scale of bulk phase separation is of the order $\left|\chi-\chi_{S}\right|^{1 / 2}$, and so the simplicity of the theory is that we can make predictions about film evolution based on the thickness of the film with respect to the length scale of phase separation: if the film is of the thickness of $\left|\chi-\chi_{S}\right|^{1 / 2}$, we might expect lateral phase separation via a transient wetting layer, since we can be reasonably certain that the film would initially vertically separate into a bilayer; if the film thickness is many times $\left|\chi-\chi_{S}\right|^{1 / 2}$, we might expect some sort of multiple layering (an oscillatory profile) and bulk-like phase separation away from the film surfaces.

To what extent can the phase portraits of chapter 3 predict the results of the simulations in chapters 4 and 5? Throughout, I have restricted to studying lateral phase separation via a transient wetting layer, in which case the films in the simulations can be considered 'thin' and have reached the equilibrium states predicted by the phase portraits. The phase portraits also help explain the particular way in which lateral phase separation via a transient wetting layer occurs in terms of phase equilibria, as shown in chapter 4 . I have not included a study of thick films that form oscillatory profiles or undergo bulk-like phase separation. What can the phase portraits and phase equilibria say about 'thick' films, with thickness many times $\left|\chi-\chi_{S}\right|^{1 / 2}$ ? The behaviour of the equilibria in the limit that the thickness goes to infinity can be easily determined from the phase portraits, by considering the film profile when part of the corresponding 
phase portrait approaches a fixed point in phase space, as discussed in chapter 3. When thicker films don't reach the equilibria predicted by the phase portraits, due to bulk-like phase separation for example, this can be considered a kinetic phenomena, since the predicted phase equilibria are still the lowest energy states of the film.

Bulk-like phase separation in films could possibly be avoided by experimental conditions. This may be important to bear in mind because lateral phase separation via a transient wetting layer produces a very neat laterally segregated structure. It might be instructive for experimentalists to consider that, if a neatly laterally segregated structure is desired, it may be best to achieve this with lateral phase separation via a transient wetting layer, rather than allow bulk-like phase separation to 'lock-out' the possibility of reaching a bilayer state and the following neat laterally segregated structure. This could perhaps be done by deliberately controlling the rate of solvent evaporation e.g. maintaining a high solvent concentration for an extended period of time prior to gradual removal of solvent, which might result in a bilayer initially forming (the increased miscibility from solvent could be used to tune the length scale of phase separation to the order of the film thickness) followed by lateral phase separation as solvent is removed. This could be important when tailoring morphologies to create a better functioning polymer-blend thin film solar cell.

The current form of the 3D model of coupled phase separation and dewetting that I developed in chapter 5 is perhaps only applicable to thin films undergoing non-bulk-like phase separation, such as lateral phase separation via a transient wetting layer. This is because of the averaging used in the model, both in the way that material is moved laterally and in the way that averaging is used to calculate lateral flux in the dewetting stage of the model. However, since the dewetting process I have discussed is primarily applicable to thin fluid films, perhaps the model is not applicable to films that are thick enough to display bulk-like phase separation. The results of chapter 3 would predict that an antisymmetric film would have roughened differently, and so the results of chapter 5 highlight the importance of kinetic considerations in determining the final roughened morphology.

The surface energy I have used throughout this thesis acts locally at the film surfaces, so it is not clear how results might change if a different form of the surface field (e.g. one that extends a finite distance into the film) might lead to different findings. An approximate idea of surface energies would allow the plotting of simple qualitative phase portraits and a prediction of the sort of equilibria that might be expected to occur. The work of chapter 4 would suggest that we can simply generalise surface energy regimes to a few cases, and therefore understand the way phase separation generally proceeds in each regime.

I will briefly suggest possible future work that might be of interest. It might be instructive to investigate more bulk-like phase separation in thicker films, to see how the idea about phase equilibria determining film evolution (as per thin films) performs alongside bulk-like behaviour, and what structures form as a result of this. In that case, the inclusion of solvent, and particularly the use of 3D simulations, would be very important. 
Given more understanding of how thicker films evolve, it would also be instructive to include solvent evaporation, and a systematic investigation of how this affects the resulting structures. This may help us understand how removal of solvent in the spin-coating process can be used to control the final morphology. This might be especially important in trying to obtain desired structures in thicker films. These sorts of investigations, combined with knowledge of how phase equilibria determine the stages of phase separation, might prove very useful in predicting and controlling film evolution and morphology. Since the depth profile of films cannot be measured during spin-coating, theoretical predictions may be a good way to reveal what is happening within the film, based on what can be observed during the process and on the final morphology.

Another possible route for study, in a different direction, would be the use of the structures produced from the simulations described above in simulations of charge transport, as a means of determining, and perhaps cataloguing, which types of structures lead to better performing photovoltaic films. This is essentially a percolation problem: charges created in the film need to be able to navigate through the phase separated structure to reach the electrodes at the film surfaces. The phase separated structure, both in the centre of the film and at the film surfaces, is therefore extremely important. It is not clear which morphologies give better performances or why, and so combining understanding of this with understanding of how to tailor morphologies, as mentioned above, could be extremely promising.

In this thesis, I have explored the phase equilibria of polymer-blend thin films using a Hamiltonian phase portrait method, and used phase portraits and simulations to identify that phase equilibria calculated in 1D can explain the evolution of films in the case of lateral phase separation via a transient wetting layer. Using a 3D model of coupled phase separation and dewetting, I have demonstrated pattern formation whereby surface roughening shadows underlying phase separation. The overall theme of this thesis is then simply this: a polymer-blend thin film will evolve to lower its free energy, and most of the behaviour can be explained entirely by evolution to and between different equilibria. I hope that my research contributes to understanding of the fundamentals of phase separation in polymer blend thin films. 


\section{Appendices}

A Diffusion Simulations on GPUs with CUDA 133

A.1 Principles of Parallelised GPU code . . . . . . . . . . . . . . 133

A.2 Steps of Simulation . . . . . . . . . . . . . . . . . . 134

A.3 Improving Efficiency . . . . . . . . . . . . . . . . . . 137

B Functional Derivatives for dewetting model 141

B.1 'Continuous derivation' . . . . . . . . . . . . . . . . . . . 141

B.2 'Discrete derivation' . . . . . . . . . . . . . . . . . . . . . . . 145

C Numerical implementation for 3D dewetting model 149

C.1 Lateral Transport Step . . . . . . . . . . . . . . . . . . . . 150

C.2 Diffusion Step . . . . . . . . . . . . . . . . . . . . . . . 152 



\section{Appendix A}

\section{Diffusion Simulations on GPUs with CUDA}

I implemented my simulations in CUDA, a programming language extension to $\mathrm{C}++$ that can be used to write code that can be executed on a CUDA-enabled Graphical Processing Unit (GPU). A GPU allows thousands of tasks to be performed in parallel, with different threads performing each task. I have written this section to assist somebody writing GPU code for a diffusion simulation of the sort given in this thesis. Rather than go into too many details of GPU programming, I will try to cover some general principles for efficient parallelised GPU code focussing on the simulations used for chapter 4.

\section{A.1 Principles of Parallelised GPU code}

\section{Avoid Branching in Code}

GPU code is most efficient when all the tasks to be done are identical, such that each thread is executing the same code but with 'different numbers' e.g. different values of $\phi_{i j}$. This point is important, since it means that branching in code should be minimal i.e. there should be as few if-else statements (which cause parrallel threads to execute different code) as possible. The reason for this is: if 10 parallel tasks encounter an if-else branch in code and 5 tasks follow 'if' and the other 5 tasks follow 'else', in fact all 10 tasks follow both branches, which therefore takes twice the amount of time that would be expected. Only if all 10 tasks follow only 'if' or only 'else' do the tasks not have to do twice the work (note that this point about branching concerns only threads within the same block).

\section{Application of Boundary Conditions}

Because of this, when boundary conditions such as 'no material flux through a surface' have to be enforced during a simulation involving 'moving material around', it is better to separate the tasks of 'enforcing no material flux through a surface' and 'moving material around' rather than to implement a boundary condition of 'no material flux through a surface' while 'moving material around' (which would require more branching in code). So the general principle is to 
separate steps that enforce numerical boundary conditions from steps which then use those boundary conditions, in order to avoid branching. This also simplifies code, making it easier to write optimised parallelised code; the best optimisation (memory retrieval etc.) for 'moving material around' may not be the best optimisation for enforcing 'no material flux through a surface'.

\section{A.2 Steps of Simulation}

My simulations required storage of both the volume fraction and the chemical potential, each set of values being stored on a separate array on the GPU i.e. two arrays (it is better to have structures-of-arrays than arrays-of-structures, for more efficient memory access with GPUs). My simulations were broken down into 4 sub-steps, which were performed for every time-step in the simulation:

1. Apply 'central-differencing at surfaces' boundary conditions;

2. Calculate the chemical potential for every grid cell;

3. Apply 'no flux through surfaces' boundary conditions;

4. Update values of $\phi$ for every grid cell.

\section{1: Central-differencing at surfaces}

This section is concerned with calculating gradients of the volume fraction in the vertical direction i.e. $\nabla_{z} \phi$. The first-order central-differencing scheme used in my simulations was

$$
\begin{aligned}
\nabla_{c} \phi_{i} & =\left(\frac{1}{2} \phi_{i+1}-\frac{1}{2} \phi_{i-1}\right) / \Delta z, \\
\nabla_{c}^{2} \phi_{i} & =\left(\phi_{i+1}-2 \phi_{i}+\phi_{i-1}\right) /(\Delta z)^{2},
\end{aligned}
$$

where the $z$-direction between the walls is discretised with index $i$ (this central differencing scheme was also used to calculate gradients in chemical potential, explained later, and also for the lateral dimension).

Figure A.1 shows a schematic of the simulation grid: the bold lines represent the surfaces at $z=0$ and $z=d$, the grid cells containing the results are those between these surfaces i.e from $i=1$ to $i=D$. As shown in the schematic, there are actually two more layers of 'virtual' grid-cells (shaded) beyond the surfaces, so the array was actually $D+2$ wide. This was also true of the array storing the chemical potential, but I will only discuss the relevance to $\phi$ for now.

To calculate $\nabla_{c} \phi_{2}$ or $\nabla_{c}^{2} \phi_{2}$, the gradients in 'bulk' cells not directly adjacent to the surface, is very simple, requiring a simple application of equation (A.1) for $i=2$. The sets of three shaded cells between the film surfaces represents the cells required for this calculation. But how does one calculate the gradient for $i=1$, for cells directly adjacent to the surface at $z=0$ ? Technically, there is no cell at $i=0$, since all of the polymer fluid is within the impenetrable walls; the sets of three shaded cells which overlap the film surfaces represent this problem. 
This point is usually, and frustratingly, glossed over, so I present my solution to the problem here. For the $i=1$ cells next to the surface, consider the central differencing scheme for the first derivative for the cell $i=1$ :

$$
\nabla_{c} \phi_{1}=\left(\frac{1}{2} \phi_{2}-\frac{1}{2} \phi_{0}\right) / \Delta z
$$

and also consider the forward differencing scheme for the first derivative, to second order accuracy:

$$
\nabla_{f} \phi_{1}=\left(-\frac{3}{2} \phi_{1}+2 \phi_{2}-\frac{1}{2} \phi_{3}\right) / \Delta z
$$

If we set $\nabla_{c} \phi_{1}=\nabla_{f} \phi_{1}$ then we find that if we set

$$
\phi_{0}=+3 \phi_{1}-3 \phi_{2}+\phi_{3} .
$$

then the result of the central difference equation (A.3) using $\phi_{0}$ is the same as doing forward-differencing to second order accuracy, which is a very nice result (since we help nullify the use of forward differencing by having increased the accuracy of this derivative). Similarly for the $z=D$ wall, we obtain

$$
\phi_{D+1}=+3 \phi_{D}-3 \phi_{D-1}+\phi_{D-2} .
$$

The advantage of setting the 'virtual' cells at $i=0$ and $i=D+1$ to expressions in (A.5) and (A.6) respectively is that the central differencing regimes in equation (A.1) can be applied to the cells at $i=1$ and $i=D$, as if these cells were not any different from grid cells 'in the bulk' of the film (this avoids the need to obtain from memory the 'next-nearest neighbour' cells $\phi_{3}$ and $\phi_{D-2}$, which would otherwise be required to use second-order forwards and backwards differencing in

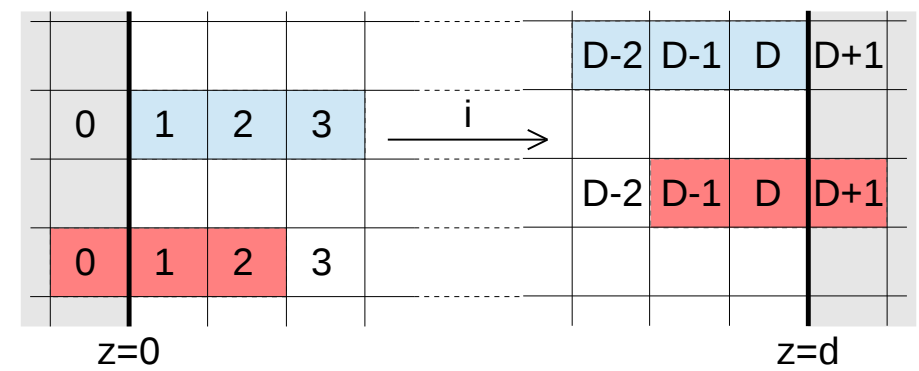

Figure A.1: Simulation grid. The surface/walls of the film are given by the bold lines. Between the bold lines are the parts of the grid representing the cells $i=1 . . D$, which are the cells representing the polymer blend i.e. the cells containing the simulation results. Beyond the surfaces are 'virtual cells' $i=0$ and $i=D+1$, which are also stored in memory in the same array as the cells between the walls. The sets of three shaded cells are for visualisation of the cells required for central-differencing in the vertical direction: central differencing for 'bulk' cells is simple $(i=2$ and $i=D-1)$, but how does one do central differencing for cells adjacent to the surfaces $(i=1$ and $i=D)$ ? See main text above. 
step (ii) of the simulation). It can easily be verified that central differencing for $\nabla_{c}^{2}$ in equation (A.1) using $\phi_{0}$ is equivalent to first order forward differencing $\nabla_{f}^{2}$ (similarly for backward difference using $\phi_{D+1}$ ).

These substitutions, which are essentially numerical boundary conditions applied prior to calculations involving the gradient, are useful for speeding up and simplification of GPU code. The same idea can be extended to higher-order differencing schemes.

\section{2: Calculate the chemical potential for every grid cell}

Comparing the governing diffusion equation (4.24) with the general diffusion equation (4.21) reveals that we can write the governing diffusion equation in the following simplified form:

$$
\frac{\partial \phi_{i j}}{\partial \tau}=\frac{1}{N} \nabla^{\prime 2} \mu_{i j}^{\prime \prime}
$$

where the scaled chemical potential in each grid cell, which I have denoted $\mu_{i j}^{\prime \prime}$ since several scaling factors have been absorbed into this expression, is therefore given by

$$
\begin{aligned}
\mu_{i j}^{\prime \prime} & =\left.\frac{1}{\left|\chi-\chi_{S}\right|} \frac{\partial f_{F H}}{\partial \phi}\right|_{i j}+\frac{\left(1-2 \phi_{i j}\right)}{\phi_{i j}\left(1-\phi_{i j}\right)} \frac{\kappa_{i j}}{a^{2}}\left(\left.\nabla^{\prime} \phi\right|_{i j}\right)^{2}-\left.2 \frac{\kappa_{i j}}{a^{2}} \nabla^{\prime 2} \phi\right|_{i j} \\
& +\frac{\delta_{i D}}{\Delta z^{\prime}}\left[\frac{a^{-1}}{\left|\chi-\chi_{S}\right|^{\frac{1}{2}}} \frac{\partial f_{d}}{\partial \phi_{d}}+\left.2 \frac{\kappa_{D j}}{a^{2}} \nabla_{z}^{\prime} \phi\right|_{D j}\right] \\
& +\frac{\delta_{i 1}}{\Delta z^{\prime}}\left[\frac{a^{-1}}{\left|\chi-\chi_{S}\right|^{\frac{1}{2}}} \frac{\partial f_{0}}{\partial \phi_{0}}-\left.2 \frac{\kappa_{1 j}}{a^{2}} \nabla_{z}^{\prime} \phi\right|_{1 j}\right] .
\end{aligned}
$$

If we calculate and store the values $\mu_{i j}^{\prime \prime}$, then we can easily apply the central differencing operator $\nabla^{\prime 2}$ to these values to calculate equation (A.7). This means that we only need to calculate $\mu_{i j}^{\prime \prime}$ from equation (A.8) once per time step. If we tried to calculate $\partial \phi_{i j} / \partial \tau$ without this intermediate step, for example by applying equation (4.24) without storing the value in the round brackets (i.e. $\mu_{i j}^{\prime \prime}$ ), then we would end up actually calculating $\mu_{i j}^{\prime \prime}$ three times: once for each $\partial \phi_{i j} / \partial \tau$, $\partial \phi_{i+1, j} / \partial \tau$, and $\partial \phi_{i-1, j} / \partial \tau$ (actually, including the second dimension $j$, we would end up calculating this value 5 time, and seven times for three dimensions). To summarise, it is much more efficient to calculate and store values of the chemical potential, so that they can be used multiple times without requiring recalculation.

\section{3: Apply 'no flux through surfaces' boundary conditions}

In order to conserve the amount of material in the film, such that equation (4.25) is fulfilled, we can implement a simple numerical boundary condition, thanks to the virtual grid cells $i=0$ and $i=D+1$ for the grid storing values of the chemical potential. This boundary condition must be implemented after the calculation of the chemical potential $\mu^{\prime \prime}$ but before calculation of $\partial \phi_{i j} / \partial \tau$ using equation (A.7). 
This step simply consists of setting

$$
\begin{array}{r}
\mu_{i=0}=\mu_{i=1}, \\
\mu_{i=D}=\mu_{i=D+1} .
\end{array}
$$

This has the effect of making the material current across the walls equal to zero, (e.g. the material current across the $z=0$ wall is $J_{-1 / 2} \propto \mu_{i=1}-\mu_{i=0}$ ) hence material is conserved. Also, this means that every grid cell can be treated the same in step 4, and we don't have to make exceptions for grid cells adjacent to the surfaces in order to conserve material.

\section{4: Update values of $\phi$ for every grid cell}

We used a forward-differencing time-step to update $\phi$ :

$$
\phi_{i j}^{\tau+\Delta \tau}=\phi_{i j}^{\tau}+\Delta \tau \frac{\partial \phi_{i j}}{\partial \tau} .
$$

After the steps outlined above, this step is incredibly simple, simply requiring the application of equation (A.7) to calculate the change in $\phi_{i j}$, and then applying equation (A.11) to update $\phi_{i j}$. It may be worth noting that forward time differencing can be unstable, requiring fairly small time-steps, but I did not find that I had significant problems with this, and the program would have become much more complicated using a different type of time differencing method.

\section{A.3 Improving Efficiency}

\section{Use mixed precision code}

Experience with these sorts of simulations has convinced me that the variables $\phi$ and $\mu$ should be stored as double-precision floats ('doubles') to ensure material conservation (which can not be honoured over time due to accumulating numerical errors if these values are stored as floats) while calculations should all be floatingpoint precision (floating-point calculations, especially for functions like 'log' and 'exp', are several times faster than double precision equivalents, especially on GPUs).

\section{Shared Memory and 'Halo' Cells}

A slightly technical point can be made about utilising 'shared memory' on GPUs, and how to make fetches from the 'global memory' into shared memory more efficiently. The information that follows may be difficult to understand for readers not familiar with GPU programming.

Threads are actually executed in batches called blocks, which can be $2 \mathrm{D}$ e.g. if I need to do $100 \times 100$ calculations in parallel requiring $100 \times 100$ threads, I could do this using $10 \times 5$ blocks containing $10 \times 20$ threads. Note that each thread in the block has an index, in this case a $2 \mathrm{D}$ index $i j$, that identifies it within the block. If shared memory is allocated when a GPU process is executed, then 


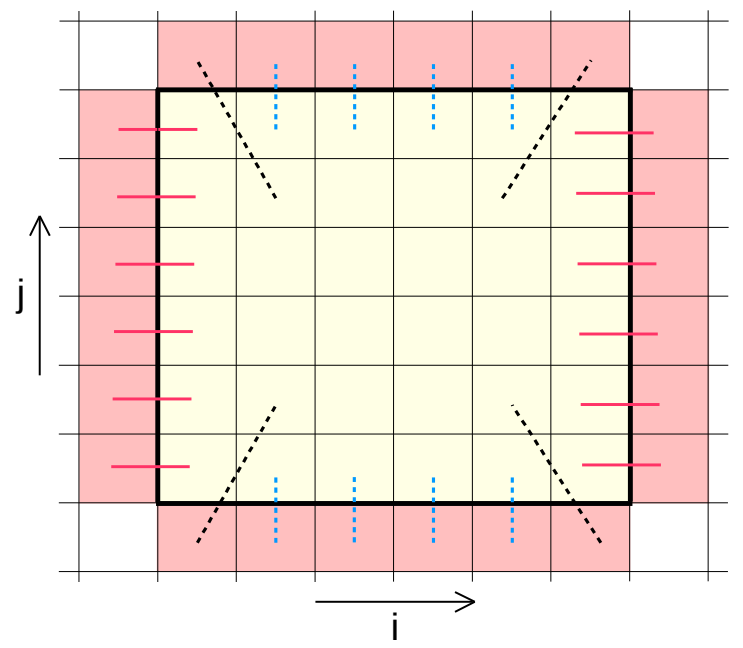

Figure A.2: Representation of a block of threads with dimensions $6 \times 6$ fetching memory from part of an array: the part of array is the $8 \times 8$ grid, and the $6 \times 6$ grid within the bold square represents the block of threads such that each thread is represented by a grid-cell in the $6 \times 6$ grid. Each thread in the block can fetch an element from the array, such that the thread with index $i j$ can fetch the array element $A_{i j}$ : this is represented by the light (yellow) shading. However, several threads need to do extra work and fetch more array elements in the 'halo' around the block of threads, these halo elements having darker (pink) shading. This is because each thread needs 'nearest-neighbour' information. The lines going from threads within the bold square to array elements beyond the square represents a possible configuration for threads fetching these extra elements, such that, in theory, no thread has to do more than two fetches. I used this configuration in my simulations. Since nearest-neighbour information is required, I gave an $X \times Y$ block of threads $(X+2) \times(Y+2)$ elements of shared memory. (Note: due to branching, it is not clear whether the configuration I have shown is significantly better than if the threads in the corners of the block fetched two 'neighbour' elements, one to either the left or right and one to either the top or bottom. I did find a small speed-up with my represented configuration, but at the expense of more complicated code.)

a block can have access to a certain amount of shared memory, and all threads within that block have access to this shared memory. The benefits of shared memory are that it is incredibly fast to read from, compared to global memory (where the arrays required to store simulation data, e.g. $\phi$ and $\mu$, would be stored in these simulations) which is very slow to read from. If data is going to be used repeatedly by a block of threads, it is often better to read from global memory as few times as possible, so storing data retrieved from global memory in shared memory, and then accessing this data via shared memory, can significantly speed up simulations by several times. Also, to mention again, all the threads within a block have access to this memory, which is very useful.

In my simulations, every thread needs access to nearest neighbour information due to the central differencing scheme e.g. a thread calculating $\mu^{\prime \prime}(i=5, j=5)$ 
needs to access $\phi(i=5, j=5)$, but also $\phi(i=4, j=5), \phi(i=6, j=5)$, $\phi(i=5, j=4)$, and $\phi(i=5, j=6)$. A $6 \times 6$ block of threads actually needs access to $(8 \times 8)-4$ values of $\phi$, and since every thread needs access to 5 values, it is much more efficient if all of the threads cooperatively read these values from global memory to shared memory. A schematic representation of this idea, showing a block of threads reading values from global memory into shared memory, is given in figure A.2, which is explained by its caption. 



\section{Appendix B}

\section{Functional Derivatives for dewetting model}

There are two slightly different approaches to calculating the functional derivatives required for chapter 5, which I will denote the 'continuous derivation' and the 'discrete derivation'. While the calculations of the functional derivatives do not have any bearing on the presented model, I feel that it is important to be explicit here in the derivations.

\section{Variations with respect to conserved quantities}

As a reminder, we must write $(\phi \Delta z) / \Delta z$ in place of $\phi$ when performing functional derivatives, such that $(\phi \Delta z)$ and $\Delta z$ can be treated as variables in there own right. To simplify this notation, I sometimes write $\psi \equiv \phi \Delta z$.

\section{B.1 'Continuous derivation'}

\section{B.1.1 The sum}

We can take the second term of equation (5.4), $\sum \Delta z g(\phi)$, and in the same vein to the work in chapter 4 , focus only on the vertical dimension $z$ (ignoring the lateral dimension $y$ for the time being) and the term in $g(\phi)$ containing the gradient, $\kappa(\phi)(\nabla \phi)^{2}$, since this is the most difficult term. Now we write $\sum \rightarrow \int d z$ (hence why I have denoted this method the 'continuous derivation'), despite the vertical grid spacing which we can then consider to be in the limit $\Delta z \rightarrow 0$, and therefore write the functional derivative of the gradient containing term in (5.4) as:

$$
\begin{aligned}
& \frac{\delta}{\delta \psi}\left[\int d z(\Delta z) \kappa(\phi)(\nabla \phi)^{2}\right]= \\
& \lim _{\epsilon \rightarrow 0} \frac{1}{\epsilon} \int d z\left[(\Delta z) \kappa\left(\frac{\psi+\epsilon p}{\Delta z}\right)\left(\nabla\left(\frac{\psi+\epsilon p}{\Delta z}\right)\right)^{2}-(\Delta z) \kappa(\phi)(\nabla \phi)^{2}\right]
\end{aligned}
$$


where $p$ is a test function (playing the role of $g\left(z, z^{\prime}\right)$ from chapter 4 ). We make several expansions:

$$
\begin{aligned}
\kappa\left(\frac{\psi+\epsilon p}{\Delta z}\right) & =\kappa\left(\frac{\psi}{\Delta z}+\frac{1}{\Delta z} \epsilon p\right) \\
& =\kappa(\phi)+\frac{1}{\Delta z}\left(\partial_{\phi} \kappa\right) \epsilon p \\
\left(\nabla\left(\frac{\psi+\epsilon p}{\Delta z}\right)\right)^{2} & =\left(\nabla\left(\frac{\psi}{\Delta z}+\frac{1}{\Delta z} \epsilon p\right)\right)^{2} \\
& =\left(\nabla \phi+\nabla\left(\frac{1}{\Delta z}\right) \epsilon p+\frac{1}{\Delta z} \epsilon \nabla p\right)^{2} \\
& =(\nabla \phi)^{2}+2 \nabla \phi\left(\nabla\left(\frac{1}{\Delta z}\right) \epsilon p+\frac{1}{\Delta z} \epsilon \nabla p\right) \\
& =(\nabla \phi)^{2}+2 \epsilon \nabla \phi\left(-\frac{1}{\Delta z^{2}} \nabla(\Delta z) p+\frac{1}{\Delta z} \nabla p\right) .
\end{aligned}
$$

Inserting these expansions into equation (B.1), part way through the calculation we arrive at

$$
\begin{aligned}
& \frac{\delta}{\delta \psi}\left[\int d z(\Delta z) \kappa(\phi)(\nabla \phi)^{2}\right]= \\
& \int d z\left[p\left(-2 \frac{1}{\Delta z} \kappa(\phi) \nabla \phi \nabla h+\left(\partial_{\phi} \kappa\right)(\nabla \phi)^{2}\right)+\nabla p(2 \kappa(\phi) \nabla \phi)\right],
\end{aligned}
$$

and calculating the rightmost term, similar to the work in chapter 4 :

$$
\begin{aligned}
\int d z[\nabla p(2 \kappa(\phi) \nabla \phi)] & =[2 \kappa(\phi) \nabla \phi]_{0}^{h}-\int d z \nabla(2 \kappa(\phi) \nabla \phi) p \\
& =[2 \kappa(\phi) \nabla \phi]_{0}^{h}-\int d z p\left(2 \kappa(\phi) \nabla^{2} \phi+2\left(\partial_{\phi} \kappa\right)(\nabla \phi)^{2}\right),
\end{aligned}
$$

and then putting it all together we arrive at:

$$
\begin{aligned}
\frac{\delta}{\delta \psi}\left[\int d z(\Delta z) \kappa(\phi)(\nabla \phi)^{2}\right]= & -\left(\partial_{\phi} \kappa\right)(\nabla \phi)^{2}-2 \kappa(\phi) \nabla^{2} \phi+[2 \kappa(\phi) \nabla \phi]_{0}^{h} \\
& -\frac{2}{\Delta z} \kappa(\phi) \nabla(\Delta z) \cdot \nabla \phi
\end{aligned}
$$

The functional derivative of the term $\Delta z f_{F H}(\phi)$ in $\Delta z g(\phi)$ simply gives us $+\partial_{\phi} f_{F H}(\phi)$. Including the lateral dimension $y$ simply makes the gradient operators $2 \mathrm{D}$, so we arrive at

$$
\begin{aligned}
& \frac{\delta}{\delta \psi}\left[\int d z(\Delta z) g(\phi)\right]= \\
& +\partial_{\phi} f_{F H}(\phi)-\left(\partial_{\phi} \kappa\right)(\nabla \phi)^{2}-2 \kappa(\phi) \nabla^{2} \phi+[2 \kappa(\phi) \nabla \phi]_{0}^{h} \\
& -\frac{2}{\Delta z} \kappa(\phi) \nabla(\Delta z) \cdot \nabla \phi .
\end{aligned}
$$


A similar derivation can be done for the functional derivative with respect to $\Delta z$, beginning with:

$$
\begin{aligned}
& \frac{\delta}{\delta \Delta z}\left[\int d z(\Delta z) \kappa(\phi)(\nabla \phi)^{2}\right]= \\
& \lim _{\epsilon \rightarrow 0} \frac{1}{\epsilon} \int d z\left[(\Delta z+\epsilon p) \kappa\left(\frac{\psi}{\Delta z+\epsilon p}\right)\left(\nabla\left(\frac{\psi}{\Delta z+\epsilon p}\right)\right)^{2}-(\Delta z) \kappa(\phi)(\nabla \phi)^{2}\right],
\end{aligned}
$$

with the result being

$$
\begin{aligned}
& \frac{\delta}{\delta \Delta z}\left[\int d z(\Delta z) g(\phi)\right]=+f_{F H}(\phi)+\kappa(\phi)(\nabla \phi)^{2} \\
& -\phi \partial_{\phi} f_{F H}(\phi)+\phi\left(\partial_{\phi} \kappa\right)(\nabla \phi)^{2}+2 \phi \kappa(\phi) \nabla^{2} \phi-[2 \phi \kappa(\phi) \nabla \phi]_{0}^{h} \\
& +\frac{2 \phi}{\Delta z} \kappa(\phi) \nabla(\Delta z) \cdot \nabla \phi,
\end{aligned}
$$

which we can be confident in since it can be verified fairly easily that if $F=$ $\int \Delta z g(\phi, \nabla \phi) d z$, then $\delta F / \delta h=g(\phi, \nabla \phi)-\phi \delta F / \delta(\phi h)$.

\section{B.1.2 The surface energy depending on $\phi$}

Now for the $f_{S}$ part of equation (5.4), which can be written as

$$
\begin{aligned}
f_{S} & =h \phi+\frac{1}{2} g \phi^{2} \\
& =h\left(\frac{\psi}{\Delta z}\right)+\frac{1}{2} g\left(\frac{\psi}{\Delta z}\right)^{2} .
\end{aligned}
$$

We then have

$$
\begin{aligned}
\frac{\partial f_{S}}{\partial \psi} & =h\left(\frac{1}{\Delta z}\right)+\frac{1}{2} g\left(\frac{2 \psi}{(\Delta z)^{2}}\right) \\
& =\left(\frac{1}{\Delta z}\right)[h+g \phi],
\end{aligned}
$$

and

$$
\begin{aligned}
\frac{\partial f_{S}}{\partial \Delta z} & =h\left(\frac{-\psi}{(\Delta z)^{2}}\right)+\frac{1}{2} g\left(\frac{-2 \psi^{2}}{(\Delta z)^{3}}\right) \\
& =-\left(\frac{\phi}{\Delta z}\right)[h+g \phi]
\end{aligned}
$$

However, this dependence on $\Delta z$ at this point is an artefact, so I remove it, since really the surface energies really ought to act only at a surface rather than across an entire grid cell adjacent to a surface. 


\section{B.1.3 Before normalising of surface terms}

We can now write

$$
\begin{aligned}
\frac{\delta F^{\phi}}{\delta(\phi \Delta z)}= & +\partial_{\phi} f_{F H}(\phi)-\left(\partial_{\phi} \kappa\right)(\nabla \phi)^{2}-2 \kappa(\phi) \nabla^{2} \phi \\
& +[2 \kappa(\phi) \nabla \phi]_{0}^{h}+\partial_{\phi} f_{0} \delta_{0}^{z}+\partial_{\phi} f_{h} \delta_{h}^{z} \\
& -\frac{2}{\Delta z} \kappa(\phi) \nabla(\Delta z) \cdot \nabla \phi, \\
\frac{\delta F^{\phi}}{\delta \Delta z}=+ & f_{F H}(\phi)+\kappa(\phi)(\nabla \phi)^{2} \\
- & \phi \partial_{\phi} f_{F H}(\phi)+\phi\left(\partial_{\phi} \kappa\right)(\nabla \phi)^{2}+2 \phi \kappa(\phi) \nabla^{2} \phi \\
- & {[2 \phi \kappa(\phi) \nabla \phi]_{0}^{h}-\phi \partial_{\phi} f_{0} \delta_{0}^{z}-\phi \partial_{\phi} f_{h} \delta_{h}^{z} } \\
+ & \frac{2 \phi}{\Delta z} \kappa(\phi) \nabla(\Delta z) \cdot \nabla \phi .
\end{aligned}
$$

\section{B.1.4 Normalising of surface terms}

We can scale the surface terms by $(\Delta z)^{-1}$ and group the surface terms, as was done in chapter 4 . This then gives us the final equations for the chemical potentials:

$$
\begin{aligned}
\frac{\delta F^{\phi}}{\delta(\phi \Delta z)}= & +\partial_{\phi} f_{F H}(\phi)-\left(\partial_{\phi} \kappa\right)(\nabla \phi)^{2}-2 \kappa(\phi) \nabla^{2} \phi \\
& +(\Delta z)^{-1}\left[+2 \kappa(\phi) \nabla_{z} \phi+\partial_{\phi} f_{h}\right] \delta_{h}^{z} \\
& +(\Delta z)^{-1}\left[-2 \kappa(\phi) \nabla_{z} \phi+\partial_{\phi} f_{0}\right] \delta_{0}^{z} \\
& -\frac{2}{\Delta z} \kappa(\phi) \nabla(\Delta z) \cdot \nabla \phi, \\
\frac{\delta F^{\phi}}{\delta \Delta z}= & f_{F H}(\phi)+\kappa(\phi)(\nabla \phi)^{2} \\
- & \phi \partial_{\phi} f_{F H}(\phi)+\phi\left(\partial_{\phi} \kappa\right)(\nabla \phi)^{2}+2 \phi \kappa(\phi) \nabla^{2} \phi \\
- & (\Delta z)^{-1}\left[+2 \phi \kappa(\phi) \nabla_{z} \phi+\phi \partial_{\phi} f_{h}\right] \delta_{h}^{z} \\
- & (\Delta z)^{-1}\left[-2 \phi \kappa(\phi) \nabla_{z} \phi+\phi \partial_{\phi} f_{0}\right] \delta_{0}^{z} \\
+ & \frac{2 \phi}{\Delta z} \kappa(\phi) \nabla(\Delta z) \cdot \nabla \phi .
\end{aligned}
$$

\section{B.1.5 The surface energy depending on $h$}

From equation (5.5) we obtain

$$
\frac{\delta F^{h}}{\delta h}=\frac{-8 \epsilon}{h^{9}}-2 \sigma \nabla^{2} h
$$




\section{B.2 'Discrete derivation'}

Since I used a priori discretisation to include a vertical dependence on composition (equation (5.2)) it is possible that we should follow a slightly different derivation, which actually yields a slightly different result for $\delta F^{\phi} / \delta \Delta z$.

\section{B.2.1 An a priori discretised gradient}

Equation (5.4) contains the bulk energy $g(\phi)$, which contains gradient terms $\nabla \phi$. We could account for discretisation of this gradient prior to taking the functional derivative of $F^{\phi}: \nabla_{y}$ will be continuous (e.g. $\left.\nabla_{y} \phi=\partial_{y} \phi\right)$ whereas $\nabla_{z}$ will already be discrete (e.g. $\left.\nabla_{z} \phi\right|_{i}=\left(\phi_{i+1}-\phi_{i-1}\right) / 2 \Delta z$ ). To investigate this, I include 2 dimensions in the derivation, and we can first break $F^{\phi}$ into two parts, accounting for a 'continuous' part $F_{C}^{\phi}$ and a 'discrete' part $F_{D}^{\phi}$ :

$$
\begin{aligned}
F^{\phi} & =\int f^{\phi}(\phi) d y+\sum_{i=1}^{D} \int \Delta z(y) g(\phi) d y \\
& =\int f^{\phi}(\phi) d y+\sum_{i=1}^{D}\left[F_{C}^{\phi}+F_{D}^{\phi}\right],
\end{aligned}
$$

where the 'continuous' part (no discrete gradient) is

$$
F_{C}^{\phi}=\int \Delta z\left[f_{F H}(\phi)+\kappa(\phi)\left(\nabla_{y} \phi\right)^{2}\right] d y
$$

and the 'discrete' part (discrete gradient) is

$$
F_{D}^{\phi}=\int \Delta z\left[\kappa(\phi)\left(\frac{\phi_{i+1}-\phi_{i-1}}{2 \Delta z(y)}\right)^{2}\right] d y
$$

I have used central differencing for the gradient in equation (B.21), although the particular differencing is not important; that the gradient now contains $\Delta z$ in the denominator is what makes a difference.

\section{B.2.2 Continuous part of $\delta F^{\phi} / \delta(\phi \Delta z)$}

This yields the same results as before:

$$
\begin{aligned}
\frac{\delta F_{C}^{\phi}}{\delta(\phi \Delta z)}= & +\partial_{\phi} f_{F H}-\left(\partial_{\phi} \kappa\right)\left(\nabla_{y} \phi\right)^{2}-2 \kappa(\phi) \nabla_{y}^{2} \phi \\
& -\frac{2}{\Delta z} \kappa(\phi) \nabla_{y}(\Delta z) \cdot \nabla_{y} \phi, \\
\frac{\delta F_{C}^{\phi}}{\delta(\Delta z)}= & f_{F H}(\phi)+\kappa(\phi)\left(\nabla_{y} \phi\right)^{2} \\
- & \phi \partial_{\phi} f_{F H}+\phi\left(\partial_{\phi} \kappa\right)\left(\nabla_{y} \phi\right)^{2}+2 \phi \kappa(\phi) \nabla_{y}^{2} \phi \\
+ & \frac{2 \phi}{\Delta z} \kappa(\phi) \nabla_{y}(\Delta z) \cdot \nabla_{y} \phi,
\end{aligned}
$$

which is not surprising. 


\section{B.2.3 Discretised part of $\delta F^{\phi} / \delta(\phi \Delta z)$}

Since the gradient term is now expressed in terms of $\Delta z$, there is no longer an explicit gradient term in $F_{D}^{\phi}$, and so the functional derivative can be found by performing the partial derivative of the integrand with respect to the relevant variables. A similar derivation was done by Fukuda et al [64]. For $1<i<D$, 'layers' of cells that are not directly adjacent to the film surfaces, we obtain:

$$
\begin{aligned}
\frac{\delta F_{D}^{\phi}}{\delta(\phi \Delta z)} & \left.\left.=-\frac{1}{2 \Delta z}\left\{+2 \kappa\left(\phi_{i+1}\right)\left(\left.\nabla_{z} \phi\right|_{i+1}\right)\right)-2 \kappa\left(\phi_{i-1}\right)\left(\left.\nabla_{z} \phi\right|_{i-1}\right)\right)\right\}+\left(\partial_{\phi} \kappa\right)\left(\left.\nabla_{z} \phi\right|_{i}\right)^{2} \\
& =-\nabla_{z}\left\{\left.2 \kappa\left(\phi_{i}\right) \nabla_{z} \phi\right|_{i}\right\}+\left(\partial_{\phi} \kappa\right)\left(\left.\nabla_{z} \phi\right|_{i}\right)^{2} \\
& \approx-2 \kappa(\phi) \nabla_{z}^{2} \phi-\left(\partial_{\phi} \kappa\right)\left(\nabla_{z} \phi\right)^{2} .
\end{aligned}
$$

The second line expresses the first line using central differencing. In the last step, I have approximated the result of the second-to-last step, which replaces the terms derived from the discretised gradient with the terms that would have been derived from a continuous gradient. It can be seen that the last two lines are equivalent to each other, apart from artefacts left over from the initial discretisation. I thus dispense of these artefacts, so that we may better approximate the real system and do away with numerical problems that appear when using the discretised result (which, I have observed, usually lead to numerical decoupling).

However, what do we do at the boundary of summation, in the cells $i=1$ and $i=D$ ? Performing the functional derivative in the cells $i=D$, we obtain:

$$
\begin{aligned}
\left.\frac{\delta F_{D}^{\phi}}{\delta(\phi \Delta z)}\right|_{D}= & \left.-\frac{1}{2 \Delta z}\left\{-2 \kappa\left(\phi_{D-1}\right)\left(\left.\nabla_{z} \phi\right|_{D-1}\right)\right)\right\}+\left(\partial_{\phi} \kappa\right)\left(\left.\nabla_{z} \phi\right|_{D}\right)^{2} \\
= & \left.-\frac{1}{2 \Delta z}\left\{+2 \kappa\left(\phi_{D+1}\right)\left(\left.\nabla_{z} \phi\right|_{D+1}\right)-2 \kappa\left(\phi_{D-1}\right)\left(\left.\nabla_{z} \phi\right|_{D-1}\right)\right)\right\} \\
& +\kappa\left(\phi_{D+1}\right)\left(\left.\nabla_{z} \phi\right|_{D+1}\right)+\left(\partial_{\phi} \kappa\right)\left(\left.\nabla_{z} \phi\right|_{D}\right)^{2} \\
= & -\nabla_{z}\left\{\left.2 \kappa\left(\phi_{D}\right) \nabla_{z} \phi\right|_{D}\right\}+\left(\partial_{\phi} \kappa\right)\left(\left.\nabla_{z} \phi\right|_{D}\right)^{2}+\kappa\left(\phi_{D+1}\right)\left(\left.\nabla_{z} \phi\right|_{D+1}\right) \\
\approx & -2 \kappa(\phi) \nabla_{z}^{2} \phi-\left(\partial_{\phi} \kappa\right)\left(\nabla_{z} \phi\right)^{2}+\kappa\left(\phi_{D+1}\right)\left(\left.\nabla_{z} \phi\right|_{D+1}\right),
\end{aligned}
$$

where I have introduced terms for 'virtual grid-cells' at $i=D+1$. However, we can't leave these grid-cells in the final answer, we should remove these artefacts of the discretisation (and include the missing factor of 2 in the final term that has disappeared because central differencing has a factor of 2 in the denominator) and keep the continuous result. Using a similar argument for the layer of grid-cells at $i=1$, and combining the answers for $i=1$ and $i=D$ with the answer for the cells $1<i<D$, we obtain:

$$
\frac{\delta F_{D}^{\phi}}{\delta(\phi \Delta z)}=-2 \kappa(\phi) \nabla_{z}^{2} \phi-\left(\partial_{\phi} \kappa\right)\left(\nabla_{z} \phi\right)^{2}+\left[2 \kappa(\phi) \nabla_{z} \phi\right]_{0}^{D} .
$$

Therefore the terms $\delta F_{D}^{\phi} / \delta(\phi \Delta z)$ are the same as in the first derivation.

\section{B.2.4 Discretised part of $\delta F^{\phi} / \delta(\Delta z)$}

For the derivative of the discretised part with respect to $\Delta z$, we will not obtain the same result, because $\Delta z$ is present in the denominator of the discretised gradient. 
We obtain for cells $1<i<D$

$$
\begin{aligned}
\frac{\delta F_{D}^{\phi}}{\delta(\Delta z)} & =\frac{\partial}{\partial \Delta z}\left(\Delta z\left[\kappa(\phi)\left[\frac{\phi_{i+1}(y)-\phi_{i-1}(y)}{2 \Delta z}\right]^{2}\right]\right) \\
& =\frac{\partial}{\partial \Delta z}\left(\Delta z\left[\kappa(\phi)\left[\frac{\psi_{i+1}(y)-\psi_{i-1}(y)}{2(\Delta z)^{2}}\right]^{2}\right]\right) \\
& =-3 \kappa(\phi)\left(\nabla_{z} \phi\right)^{2}-\phi\left(\partial_{\phi} \kappa\right)\left(\nabla_{z} \phi\right)^{2} \\
& =\kappa(\phi)\left(\nabla_{z} \phi\right)^{2}-4 \kappa(\phi)\left(\nabla_{z} \phi\right)^{2}-\phi\left(\partial_{\phi} \kappa\right)\left(\nabla_{z} \phi\right)^{2}
\end{aligned}
$$

and, if we make the leap of faith (necessary for the model to reproduce calculated equilibria when film height is held constant; see chapter 4) to include the surface terms produced from a continuous gradient in the vertical direction (the different terms obtained here are a result of the gradient changing as $\Delta z$ changes, and since the surfaces terms should act only at the surface, and not across a volume of fluid, it seems sensible to keep the continuous result that take into account that the surface terms are completely local) we obtain

$$
\begin{aligned}
\frac{\delta F_{D}^{\phi}}{\delta(\Delta z)}= & \kappa(\phi)\left(\nabla_{z} \phi\right)^{2}-4 \kappa(\phi)\left(\nabla_{z} \phi\right)^{2}-\phi\left(\partial_{\phi} \kappa\right)\left(\nabla_{z} \phi\right)^{2} \\
& -\left[2 \phi \kappa(\phi) \nabla_{z} \phi\right]_{0}^{D} .
\end{aligned}
$$

\section{B.2.5 Scaling and final equations}

For $\delta F^{\phi} / \delta(\phi \Delta z)$ we have the same result as before:

$$
\begin{aligned}
\frac{\delta F^{\phi}}{\delta(\phi \Delta z)}= & +\partial_{\phi} f_{F H}(\phi)-\left(\partial_{\phi} \kappa\right)(\nabla \phi)^{2}-2 \kappa(\phi) \nabla^{2} \phi \\
& +(\Delta z)^{-1}\left[+2 \kappa(\phi) \nabla_{z} \phi+\partial_{\phi} f_{h}\right] \delta_{h}^{z} \\
& +(\Delta z)^{-1}\left[-2 \kappa(\phi) \nabla_{z} \phi+\partial_{\phi} f_{0}\right] \delta_{0}^{z} \\
& -\frac{2}{\Delta z} \kappa(\phi) \nabla(\Delta z) \cdot \nabla \phi,
\end{aligned}
$$

but for $\delta F^{\phi} / \delta(\Delta) z$ the result is modified:

$$
\begin{aligned}
\frac{\delta F^{\phi}}{\delta \Delta z}= & +f_{F H}(\phi)+\kappa(\phi)(\nabla \phi)^{2} \\
& -\phi \partial_{\phi} f_{F H}(\phi)+\phi\left(\partial_{\phi} \kappa\right)\left(\nabla_{y} \phi\right)^{2}+2 \phi \kappa(\phi) \nabla_{y}^{2} \phi \\
& -4 \kappa(\phi)\left(\nabla_{z} \phi\right)^{2}-\phi\left(\partial_{\phi} \kappa\right)\left(\nabla_{z} \phi\right)^{2} \\
& -(\Delta z)^{-1}\left[+2 \phi \kappa(\phi) \nabla_{z} \phi+\phi \partial_{\phi} f_{h}\right] \delta_{h}^{z} \\
& -(\Delta z)^{-1}\left[-2 \phi \kappa(\phi) \nabla_{z} \phi+\phi \partial_{\phi} f_{0}\right] \delta_{0}^{z} \\
& +\frac{2 \phi}{\Delta z} \kappa(\phi) \nabla(\Delta z) \cdot \nabla \phi .
\end{aligned}
$$

Since $\nabla_{z}(\Delta z)=0$ in the model, I have written $\nabla(\Delta z) \cdot \nabla \phi$ using 3D gradient operators. The surface energy depending on film height can be calculated as in 
the continuous derivation, giving equation (B.17). It is the expressions above resulting from performing the functional derivative on a discretised functional that have been used to test the model in this chapter. This method is more consistent with how we have discretised the free energy, and should also account for a reduction in the energy cost of vertical composition gradients as the film becomes thicker in places, and vice versa. 


\section{Appendix C}

\section{Numerical implementation for $3 \mathrm{D}$ dewetting model}

Most of the general principles behind the implementation of my simulations in CUDA, to utilise a CUDA-enabled Graphical Processing Unit (GPU), are discussed in Appendix A, but here I will outline a few key points relevant to the simulations of chapter 5 . The particular implementation I used conserved material (of course) and kept the program simple, but there may very well be better implementations. Also, although I scaled the equations, I have not remarked at all on this scaling in this section, since it would confuse the matter.

Correct implementation of the rate equations (5.7), (5.8) and (5.9) and timestep is crucial for material conservation to be honoured; the governing equations of course conserve material, but it can be easy to misinterpret the equations upon implementation. In my implementation I stored values of $h(y)$ and $\phi(i, y)$, although one could probably store the values $h(y)$ and $\phi(i, y) \Delta z(y)$ instead.

\section{Suggestion for $2 \mathrm{D}$ simulations $\rightarrow 3 \mathrm{D}$ simulations}

In order to make use of shared memory effectively, in the same way discussed in Appendix A, it seemed that the best way to run 3D simulations was to divide the simulation up into 2D slices. When launching a process (kernel) on the GPU, I would then loop over these $2 \mathrm{D}$ slices. This was also the simplest way to turn a $2 \mathrm{D}$ simulation into a 3D simulation. This made the program simpler, and made memory access from global memory and storage of values using shared memory much more efficient and manageable.

While calculating values for a set of simulation grid cells within a $2 \mathrm{D}$ slice of the array, values corresponding to the previous, current and next $2 \mathrm{D}$ slices can be stored in shared memory, and current slice $\rightarrow$ previous slice and next slice $\rightarrow$ current slice as the $3 \mathrm{D}$ array is traversed by stepping through the $2 \mathrm{D}$ layers. This means we can efficiently use memory and make efficient memory accesses. I would highly recommend implementing a $3 \mathrm{D}$ simulation in a similar way. 


\section{C.1 Lateral Transport Step}

The equations governing the lateral transport step in which material is moved laterally such that the height of the film can change, equations (5.7) and (5.8), involve the transfer of material in the form of $h(y)$ and $\bar{\phi}(y) h(y)$, which together specify the amount of, and type of, fluid that is transferred.

\section{C.1.1 Explicit definition of Material Currents}

To keep track of everything and make sure that material conservation is locally correct, it is easier to explicitly introduce material currents $\vec{J}$. In $3 \mathrm{D}$ there are two components to these currents (for the lateral transport step there is only the lateral movement of material, so the currents I define here don't have a vertical component) though my discussion will only discuss one component for simplicity. These material currents can be defined at the centre of a grid cell $j$ (i.e. $J_{j}$ ) or between grid cells $j$ and $j+1$ (i.e. $J_{j+1 / 2}$ ) (note that by grid cell I mean the result of discretising the system so that it can be simulated on a computer). The lateral grid has a spacing $\Delta y$, whilst the vertical grid (arising already from the a priori discretisation of the film) has spacing $\Delta z(j)$ (where the lateral spacial variable $y$ has been replaced by a discrete index $j$ ):

Assuming that the chemical potentials have already been calculated from equations (5.11) and (5.12) (as discussed in Appendix A, it is best to calculate and store the chemical potentials before using them. Calculation and storage of the average $\bar{\phi}(j)$ in the same way is also recommended) we can define the following currents:

$$
\begin{aligned}
\nabla^{*} \mu_{c}(j) & \equiv \nabla_{j} \mu_{h}(j)+\bar{\phi}(j) \frac{1}{D} \sum_{i=1}^{D} \nabla_{j} \mu_{\phi \Delta z}(i, j) \\
& \equiv-J_{h}^{*}(j)-J_{\bar{\phi} \Delta z}^{*}(j), \\
J_{h}^{*}(j) & \equiv-\nabla_{j} \mu_{h}(j), \\
J_{\bar{\phi} \Delta z}^{*}(j) & \equiv-\bar{\phi}(j) \frac{1}{D} \sum_{i=1}^{D} \nabla_{j} \mu_{\phi \Delta z}(i, j),
\end{aligned}
$$

where $\nabla_{j} \mu_{\phi \Delta z}(i, j) \equiv\left[\mu_{\phi \Delta z}(i, j+1)-\mu_{\phi \Delta z}(i, j-1)\right] / 2 \Delta y$ for example i.e. central differencing. We can then define the currents

$$
\begin{aligned}
J_{h}(j) & \equiv \frac{h(j)^{3}}{3 \eta} J_{h}^{*}, \\
J_{\bar{\phi} \Delta z}(j) & \equiv \frac{h(j)^{3}}{3 \eta} J_{\bar{\phi} \Delta z}^{*},
\end{aligned}
$$

and then

$$
\begin{aligned}
\frac{\partial h(j)}{\partial t} & =-\nabla_{j} \cdot\left(J_{h}+J_{\bar{\phi} \Delta z}\right), \\
\frac{\partial \bar{\phi}(j) h(j)}{\partial t} & =-\nabla_{j} \cdot\left(\bar{\phi} J_{h}+\bar{\phi} J_{\bar{\phi} \Delta z}\right) .
\end{aligned}
$$


Using central differencing on the latter equations, we then obtain

$$
\begin{aligned}
\frac{\partial h(j)}{\partial t} & =-\frac{J_{h}(j+1)-J_{h}(j-1)}{2 \Delta y}-\frac{J_{\bar{\phi} \Delta z}(j+1)-J_{\bar{\phi} \Delta z}(j-1)}{2 \Delta y}, \\
\frac{\partial \bar{\phi}(j) h(j)}{\partial t} & =-\frac{\bar{\phi}(j+1) J_{h}(j+1)-\bar{\phi}(j-1) J_{h}(j-1)}{2 \Delta y} \\
& -\frac{\bar{\phi}(j+1) J_{\bar{\phi} \Delta z}(j+1)-\bar{\phi}(j-1) J_{\bar{\phi} \Delta z}(j-1)}{2 \Delta y} .
\end{aligned}
$$

\section{C.1.2 Forward-Difference Time-Step}

The values for the rate of change of material can be calculated with the equations above. To update the values of $h(j)$ and $\phi(i, j)$ such that matter is conserved, we can perform the following

$$
\begin{gathered}
{[\bar{\phi}(t+d t) h(t+d t)]=\bar{\phi}(t) h(t)+d t \frac{\partial \bar{\phi} h}{\partial t},} \\
h(t+d t)=h(t)+d t \frac{\partial h}{\partial t}, \\
\bar{\phi}(t+d t)=[\bar{\phi}(t+d t) h(t+d t)] / h(t+d t),
\end{gathered}
$$

such that we can, in this order, store the values $[\bar{\phi}(t+d t) h(t+d t)]$ in the array for $\phi$, update the values in the height array, and then convert the $\phi$ array back to storing $\phi$ values, in such a way that material is correctly conserved.

Note that the steps above concern the change of $h$ and $\bar{\phi}$ : the change in $\Delta z$ is implied very simply through $\Delta z=h / D$ where $D$ is the number of grid cells in the vertical dimension. To account for a change in $\bar{\phi}(y)$, the cells $\phi(i, y)$ must change. This is done very simply by evenly distributing the amount of material that is moved in the lateral step amongst the grid cells $i$ : if the average amount of material over grid cells $i$ at $y$ must change, then changing the amount of material in each grid cell $i$ at $y$ by how much the average at $y$ changes will account for this. Of course, it is really $[\bar{\phi} h]$ that changes and so it is this value that must be evenly distributed, as can be seen by following the steps above (equation (C.12) simply converts back to $\bar{\phi}$ for convenience). So we can implement the aforementioned equations as

$$
\begin{gathered}
{[\phi(i, y)(t+d t) \Delta z(t+d t)]=\phi(i, y)(t) \Delta z(t)+d t \frac{1}{D} \frac{\partial \bar{\phi} h}{\partial t}} \\
\Delta z(t+d t)=\Delta z(t)+d t \frac{1}{D} \frac{\partial h}{\partial t} \\
\phi(i, y)(t+d t)=\frac{[\phi(i, y)(t+d t) \Delta z(t+d t)]}{\Delta z(t+d t)}
\end{gathered}
$$




\section{C.2 Diffusion Step}

$\Delta z(j)$ doesn't change in these steps because the film height $h(j)$ doesn't change, therefore in my implementation of the diffusion step I did not find the need to explicitly calculate and store any currents (although the currents between grid cells are implicit in my implementation nonetheless).

\section{C.2.1 Diffusion from Chemical Potential}

We must implement the following equation

$$
\frac{\partial \phi(i, j) \Delta z(j)}{\partial t}=M \nabla \cdot\left(\Delta z(j) \nabla \mu_{\phi \Delta z}(i, j)\right),
$$

where the gradient operator is now for both directions represented by $i$ and $j$, rather than just the lateral direction $j$. My implementation was

$$
\begin{aligned}
\frac{\partial \phi(i, j) \Delta z(j)}{\partial t} & =\frac{M}{\Delta y}\left[\frac{\left(\mu_{j+1}-\mu_{j}\right)}{\Delta y} \frac{\left(\Delta z_{j+1}+\Delta z_{j}\right)}{2}-\frac{\left(\mu_{j}-\mu_{j-1}\right)}{\Delta y} \frac{\left(\Delta z_{j}+\Delta z_{j-1}\right)}{2}\right] \\
& +\frac{M}{\Delta z}\left[+\frac{\left(\mu_{i+1}-\mu_{i}\right)}{\Delta z} \Delta z-\frac{\left(\mu_{i}-\mu_{i-1}\right)}{\Delta z} \Delta z\right],
\end{aligned}
$$

where $\left(\mu_{j+1}-\mu_{j}\right) / \Delta y$ is essentially a current between grid cells $j+1$ and $j$, and $\left(\Delta z_{j+1}+\Delta z_{j}\right) / 2$ can be thought of as the grid-cell wall through which this current passes (the average of the heights of the grid-cells $j+1$ and $j$ ). Diffusion in the vertical direction is simpler, since the lateral grid spacing $\Delta y$ is constant everywhere. Of course, the currents throught the surfaces must be set to zero. Updating the value of $\phi$ with a forward-difference time-step is then very simple, because $\Delta z(j)$ does not change in this diffusion step

$$
\phi(t+d t)=\phi(t)+d t \frac{1}{\Delta z(j)} \frac{\partial \phi(i, j) \Delta z(j)}{\partial t} .
$$

\section{C.2.2 Random Thermal Currents}

The RHS of equation (C.17) can also include random thermal currents, as in my simulations. From any particular random distribution, one can sample currents $\vec{J}^{T}$ whose components are the random numbers selected from the distribution, such that these currents are between grid cell. A noise term that can be added to (C.17) can then be written in terms of these currents as (using one component for simplicity, specifically $j$ in which direction the grid is non-uniform)

$$
\begin{aligned}
\text { noise } & =\frac{1}{\Delta y}\left[J_{j+1 / 2}^{T} \frac{\Delta z(j+1)+\Delta z(j)}{2}-J_{j-1 / 2}^{T} \frac{\Delta z(j)+\Delta z(j-1)}{2}\right] \\
& +\frac{1}{\Delta z}\left[+J_{i+1 / 2}^{T} \Delta z-J_{i-1 / 2}^{T} \Delta z\right] .
\end{aligned}
$$

Throughout the entire diffusion step, the currents through the film surfaces must be set to zero, and this includes the random thermal currents. 


\section{Terminology}

Bilayer: The film is vertically segregated into two phases (layers) with an interface between them. These phases coat the film surfaces due to preferential surface attraction.

Blend: A liquid mixture of two components (e.g. a blend of two polymers).

Coexisting Equilibria: Profiles which coexist together, describing different phases in the film, as for a laterally inhomogeneous film of two laterally segregated phases.

Dewetting: (disambiguation) process by which a fluid film will not uniformly coat a substrate, due to forces which cause areas of substrate to tend towards 'drying'; this can lead to isolated droplets of fluid, but more generally will cause some undulation of the fluid film surface.

Fixed Points: Regions of phase space that require an infinite length of film to pass through, since these points satisfy $\dot{q}=0$ (composition gradient is zero) and $\ddot{q}=0$ (rate of change of composition gradient is zero).

Independently-Existing Equilibria: Profiles which describe the film entirely and do not coexist with other profiles, as for a laterally homogeneous film.

Lateral Phase Separation: Phase separation into laterally coexisting phases, whether from an approximately homogeneous film or a bilayer film, resulting in a laterally segregated film.

Lateral Phase Separation via a Transient Wetting Layer: Vertical phase separation initially proceeds, due to preferential surface attraction, resulting in a vertically segregated film. This state is unstable, and lateral phase separation occurs, resulting in a laterally segregated film.

Laterally Segregated (film): 'column' phases with interfaces perpendicular to the surfaces.

Mean-field: Average interactions are used in place of counting up individual interactions, such that the local behaviour can be written in terms of macroscopic average properties. Works well for polymer systems, but rarely for fluids of simple molecules in which fluctuations mean the description fails.

Monolayer: The profile is approximately constant $\phi(z) \approx \bar{\phi}$ since the blend is miscible. There may be an excess of a component near the film surfaces due to preferential attraction, depleting that component in the rest of the film. 
Phase Diagram: a diagram, drawn in a space of variables such as composition and temperature, that separates regions corresponding to different stable phases with lines, which correspond to the limits of stability of these phases. e.g. for water, a phase diagram in the temperature-pressure plane separates regions of vapour, liquid and solid.

(Phase) Equilibria: profiles which minimise the free energy of the film, and therefore correspond to equilibrium. The term phase is used in analogy with bulk blends e.g. an $A$ - $B$ blend can exist as: a miscible blend (one-phase); or $A$-rich and $B$-rich phases (two-phase).

Phase Portrait: Plot of trajectories in phase space that minimise free energy.

Phase Space: A space of the variables that describe a system e.g. position and momentum, composition and composition gradient.

Pinning: Values of $\left(\phi, 2 \kappa \nabla_{z} \phi\right)$ at the film surfaces are determined by surface boundary conditions, such that, in phase space, the ends of trajectories are always pinned to these boundary conditions.

Polymer: A molecule consisting of repeated units, like a string of beads or a chain. These repeat units are called monomers. A chain segment usually refers to a single monomer, but may refer to several monomer units in order that the chain be flexible around these segments.

Profile: description of a polymer-blend by volume fraction $\phi$ as a function of spatial variables e.g. $\phi(z, y)$, where $z$ is the vertical (depth) dimension and $y$ is the lateral (parallel to substrate) dimension. Throughout this thesis the profiles are discussed primarily in $1 \mathrm{D}$ as $\phi(z)$.

Separatrix: Phase portraits that flow through fixed points in phase space. Trajectories that flow through these fixed points (these trajectories must therefore be on the separatrix) correspond to infinitely thick films, hence as (finite) films become thicker their corresponding phase portraits tend to, but don't meet, the separatrix.

Simple molecule: molecules that can be treated as spheres, because they consist of a few atoms at most and their internal structure need not be explicitly considered.

Solution: A liquid mixture of solvent (e.g. water, toluene) and solute (e.g. sugar, polymer), in which the solute is dispersed in the solvent.

State(s): (disambiguation) the overall configuration of the film, and/or the equilibria contained in that configuration e.g. a film in a laterally segregated state will consist of laterally coexisting states. Non-specific term. 
Surface Bifurcation: Mechanism explaining the dynamics of lateral phase separation via a transient wetting layer, describing how the surface values $\left(\phi, 2 \kappa \nabla_{z} \phi\right)$ of the TWL divide into two values that evolve towards those for laterally coexisting equilibria, whilst honouring the surface boundary conditions at all times.

Surface/Wall: the boundary formed by the interface between the fluid and, for example, air or a vacuum. While the terms will often be used interchangeably, a Wall is specifically meant to be a rigid planar surface, while a Surface could be non-rigid and deformable. A substrate such as a silicon wafer, on which a fluid film may rest, is therefore a wall, whereas the fluid-air boundary may be referred to as either a wall or a surface depending on the context.

Trajectory: Path through phase space e.g. path of all the points of position and momentum that a particle had as it moved.

Vertical Phase Separation: Phase separation into vertically layered phases, e.g. a bilayer, usually caused by preferential surface attraction.

Vertically Segregated (film): Layered phases with interfaces parallel to the surfaces.

Wetting: when one phase of a binary phase system entirely coats a surface, excluding the other phase from contact. Although wetting is strictly defined to mean that the latter configuration is stable and the phase coating the surface is infinitely thick in a semi- $\infty$ system (see main text throughout), the term is usually used more loosely to describe most scenarios when a phase coats a surface. 


\section{Publications}

The work in this thesis has been published/submitted as follows:

1. Sam Coveney and Nigel Clarke. "Surface roughening in polymer blend thin films by lateral phase separation: A thermodynamic mechanism." In: The Journal of Chemical Physics 137 $174901(2012)$

2. Sam Coveney and Nigel Clarke. "Breakup of a Transient Wetting Layer in Polymer Blend Thin Films: Unification with 1D Phase Equilibria." In: Physical Review Letters 111125702 (2013)

3. Sam Coveney and Nigel Clarke. "Lateral phase separation in polymer-blend thin films: Surface Bifurcation." In: Physical Review E 89062603 (2014)

4. Sam Coveney and Nigel Clarke. "Pattern Formation in Polymer Blend Thin Films: Surface Roughening couples to Phase Separation." In: Physical Review Letters 113218301 (2014) 


\section{Bibliography}

[1] M.L. Huggins. "Solutions of Long Chain Compounds". In: The Journal of Chemical Physics 9.5 (1941), p. 440.

[2] M.L. Huggins. "Some Properties of Solutions of Long-chain Compounds". In: Journal of Physical Chemistry 46.1 (1942), p. 151.

[3] P.J. Flory. "Thermodynamics of High Polymer Solutions". In: The Journal of Chemical Physics 10.1 (1942), p. 51.

[4] P.J. Flory. "Thermodynamics of Heterogeneous Polymers and Their Solutions". In: The Journal of Chemical Physics 12.11 (1944), p. 425.

[5] M.L. Huggins. "Theory of Solutions of High Polymers". In: presented at meeting of the American Chemical Society 64.7 (1942), p. 1712.

[6] G. Scatchard. "Equilibria in Non-electrolyte Solutions in Relation to the Vapor Pressures and Densities of the Components". In: Chemical Reviews 8.2 (1931), p. 321.

[7] P.J. Flory. Principles of Polymer Chemistry. Ithaca, NY, Cornell University Press, 1953.

[8] R.A.L. Jones and R.W. Richards. Polymers at Surfaces and Interfaces. Cambridge University Press, 1999.

[9] P.J. Flory. "Fifteenth Spiers Memorial Lecture. Thermodynamics of polymer solutions". In: Discussions of the Faraday Society 49 (1970), p. 7.

[10] G. Strobl. The Physics of Polymers. Springer, 2007.

[11] K. F. Freed, ed. Phase Behaviour in Polymer Blends. Springer, 2005. Chap. Critical to Mean Field Crossover in Polymer Blends.

[12] P.J. Flory. "Thermodynamics of Dilute Solutions of High Polymers". In: The Journal of Chemical Physics 13.11 (1945), p. 453.

[13] J.W. Cahn and J.E. Hilliard. "Free Energy of a Nonuniform System. I. Interfacial Free Energy". In: The Journal of Chemical Physics 28.2 (1958), p. 258.

[14] J.W. Cahn. "Free Energy of a Nonuniform System. II. Thermodynamic Basis". In: The Journal of Chemical Physics 30.5 (1959), p. 1121.

[15] J.W. Cahn and J.E. Hilliard. "Free Energy of a Nonuniform System. III. Nucleation in a Two-Component Incompressible Fluid". In: The Journal of Chemical Physics 31.3 (1959), p. 688. 
[16] J.W. Cahn. "Phase Separation by Spinodal Decomposition in Isotropic Systems". In: The Journal of Chemical Physics 42.1 (1965), p. 93.

[17] P.-G. de Gennes. Scaling Concepts In Polymer Physics. Ithaca, NY, Cornell University Press, 1979.

[18] P.-G. de Gennes. "Dynamics of fluctuations and spinodal decomposition in polymer blends". In: The Journal of Chemical Physics 72.9 (1980), p. 4756.

[19] G. Jannink and P.-G. de Gennes. "Quasielastic Scattering by Semidilute Polymer Solutions". In: The Journal of Chemical Physics 48.5 (1968), p. 2260.

[20] P.-G. de Gennes. "Theory of X-Ray scattering by liquid macromolecules with heavy atom labels". In: Journal de Physique 31 (1970), p. 235.

[21] S.F. Edwards. "The statistical mechanics of polymers with excluded volume". In: Proceedings of the Physical Society 85.4 (1965), p. 613.

[22] M. Hillert. "A solid-solution model for inhomogeneous systems". In: Acta Metallurgica 9.6 (1961), p. 525.

[23] J.W. Cahn. "On spinodal decomposition". In: Acta Metallurgica 9.9 (1961), p. 795.

[24] H.E. Cook. "Brownian motion in spinodal decomposition". In: Acta Metallurgica 18 (1970).

[25] P. Pincus. "Dynamics of fluctuations and spinodal decomposition in polymer blends. II". In: The Journal of Chemical Physics 75.4 (1981), p. 1996.

[26] K. Binder. "Collective diffusion, nucleation, and spinodal decomposition in polymer mixtures". In: The Journal of Chemical Physics 79.12 (1983), p. 6387.

[27] J.W. Cahn. "Critical point wetting". In: The Journal of Chemical Physics 66.8 (1977), p. 3667.

[28] T. Lubensky and M. Rubin. "Critical phenomena in semi-infinite systems. II. Mean-field theory". In: Physical Review B 12.9 (1975), p. 3885.

[29] R. Pandit and M. Wortis. "Surfaces and interfaces of lattice models: Meanfield theory as an area-preserving map". In: Physical Review B 25.5 (1982), p. 3226.

[30] H. Nakanishi and M. Fisher. "Multicriticality of Wetting, Prewetting, and Surface Transitions". In: Physical Review Letters 49.21 (1982), p. 1565.

[31] H. Nakanishi and P. Pincus. "Surface spinodals and extended wetting in fluids and polymer solutions". In: The Journal of Chemical Physics 79.2 (1983), p. 997.

[32] R.A. Jerry and E. Nauman. "Phase transitions in thin films of a binary mixture". In: Physics Letters A 167.2 (1992), p. 198.

[33] I. Schmidt and K. Binder. "Model calculations for wetting transitions in polymer mixtures". In: Journal de Physique 46 (1985), p. 1631. 
[34] K. Binder and D. Landau. "Capillary condensation in the lattice gas model: A Monte Carlo study". In: The Journal of Chemical Physics 96.2 (1992), p. 1444.

[35] K. Binder. "Phase transitions in reduced geometry". In: Annual Review of Physical Chemistry 43 (1992), p. 33.

[36] K. Binder. "Modeling of Wetting in Restricted Geometries". In: Annual Review of Materials Research 38.1 (2008), p. 123.

[37] T. Flebbe, B. Dünweg, and K. Binder. "Phase Separation Versus Wetting: a Mean Field Theory for Symmetrical Polymer Mixtures Confined Between Selectively Attractive Walls". In: Journal de Physique II 6.5 (1996), p. 667.

[38] A. Parry and R. Evans. "Influence of wetting on phase equilibria: A novel mechanism for critical-point shifts in films". In: Physical Review Letters 64.4 (1990), p. 439.

[39] E.V. Albano, K. Binder, D.W. Heermann, and W. Paul. "Critical wetting in the square Ising model with a boundary field". In: Journal of Statistical Physics 61.1-2 (1990), p. 161.

[40] A. Parry and R. Evans. "Novel phase behaviour of a confined fluid or Ising magnet". In: Physica A: Statistical Mechanics and its Applications 181.3-4 (1992), p. 250.

[41] M. Souche and N. Clarke. "Phase equilibria in polymer blend thin films: a Hamiltonian approach". In: The Journal of Chemical Physics 131.24 (2009), p. 244903.

[42] M. Souche and N. Clarke. "Equilibrium Phases for Thin Films of Polymer Blend Solutions". In: Macromolecules 43.12 (2010), p. 5433.

[43] K. Binder, D. Landau, and A. Ferrenberg. "Character of the Phase Transition in Thin Ising Films with Competing Walls". In: Physical Review Letters 74.2 (1995), p. 298.

[44] K. Binder, D. Landau, and A. Ferrenberg. "Thin Ising films with competing walls: A Monte Carlo study". In: Physical Review E 51.4 (1995), p. 2823.

[45] M. Müller, E. Albano, and K. Binder. "Symmetric polymer blend confined into a film with antisymmetric surfaces: Interplay between wetting behavior and the phase diagram". In: Physical Review E 62.4 (2000), p. 5281.

[46] S. Coveney and N. Clarke. "Surface roughening in polymer blend thin films by lateral phase separation: a thermodynamic mechanism". In: The Journal of Chemical Physics 137.17 (2012), p. 174901.

[47] E. Helfand. "Theory of the Interface between Immiscible Polymers. II". In: The Journal of Chemical Physics 56.7 (1972), p. 3592.

[48] E. Helfand. "Theory of unsymmetric polymerpolymer interfaces". In: The Journal of Chemical Physics 62.4 (1975), p. 1327.

[49] K.M. Hong and J. Noolandi. "Theory of inhomogeneous multicomponent polymer systems". In: Macromolecules 14.3 (1981), p. 727. 
[50] S. Coveney and N. Clarke. "Breakup of a Transient Wetting Layer in Polymer Blend Thin Films: Unification with 1D Phase Equilibria". In: Physical Review Letters 111.12 (2013), p. 125702.

[51] S. Coveney and N. Clarke. "Lateral phase separation in polymer-blend thin films: Surface bifurcation". In: Physical Review E 89.6 (2014), p. 062603.

[52] R.C. Ball and R.L.H. Essery. "Spinodal decomposition and pattern formation near surfaces". In: Journal of Physics: Condensed Matter 2 (1990), p. 10303.

[53] R.A.L. Jones, L.J. Norton, E.J. Kramer, R.J. Composto, R.S. Stein, T.P. Russell, A. Mansour, A. Karim, G.P. Felcher, M.H. Rafailovich, J. Sokolov, X. Zhao, and S.A. Schwarz. "The Form of the Enriched Surface Layer in Polymer Blends". In: Europhysics Letters 12.1 (1990), p. 41.

[54] S. Puri and K. Binder. "Surface-directed spinodal decomposition: Phenomenology and numerical results". In: Physical Review A 46.8 (1992), R4487.

[55] G. Brown and A. Chakrabarti. "Surface-directed spinodal decomposition in a two-dimensional model". In: Physical Review A 46.8 (1992), p. 4829.

[56] J. Marko. "Influence of surface interactions on spinodal decomposition". In: Physical Review E 48.4 (1993), p. 2861.

[57] S. Puri and K. Binder. "Surface effects on spinodal decomposition in binary mixtures and the interplay with wetting phenomena". In: Physical Review E 49.6 (1994), p. 5359.

[58] W. Straub, F. Bruder, R. Brenn, G. Krausch, H. Bielefeldt, A. Kirsch, O. Marti, J. Mlynek, and J.F. Marko. "Transient Wetting and 2D Spinodal Decomposition in a Binary Polymer Blend". In: Europhysics Letters 29 (1995), p. 353.

[59] S. Walheim, M. Bo, G. Krausch, and U. Steiner. "Structure Formation via Polymer Demixing in Spin-Cast Films". In: Macromolecules 30.96 (1997), p. 4995.

[60] S.Y. Heriot and R.A.L. Jones. "An interfacial instability in a transient wetting layer leads to lateral phase separation in thin spin-cast polymerblend films". In: Nature Materials 4.10 (2005), p. 782.

[61] A.D.F. Dunbar, P. Mokarian-Tabari, A.J. Parnell, S.J. Martin, M.W.A. Skoda, and R.A.L. Jones. "A solution concentration dependent transition from self-stratification to lateral phase separation in spin-cast PS:d-PMMA thin films". In: The European Physical Journal E, Soft matter 31.4 (2010), p. 369.

[62] P. Mokarian-Tabari, M. Geoghegan, J.R. Howse, S.Y. Heriot, R.L. Thompson, and R.A.L. Jones. "Quantitative evaluation of evaporation rate during spin-coating of polymer blend films: Control of film structure through defined-atmosphere solvent-casting". In: The European Physical Journal E, Soft matter 33.4 (2010), p. 283. 
[63] I.C. Henderson and N. Clarke. "On Modelling Surface Directed Spinodal Decomposition". In: Macromolecular Theory and Simulations 14.7 (2005), p. 435 .

[64] J. Fukuda, M. Yoneya, and H. Yokoyama. "Numerical treatment of the dynamics of a conserved order paramater in the presence of walls". In: Physical Review E 73 (2006).

[65] S. Das, S. Puri, J. Horbach, and K. Binder. "Kinetics of phase separation in thin films: Simulations for the diffusive case". In: Physical Review E 72.6 (2005).

[66] G.A. Buxton and N. Clarke. "Ordering polymer blend morphologies via solvent evaporation". In: Europhysics Letters 78.5 (2007), p. 56006.

[67] S. Coveney and N. Clarke. "Pattern Formation in Polymer Blend Thin Films: Surface Roughening Couples to Phase Separation". In: Physical Review Letters 113 (2014), p. 218301.

[68] N. Clarke. "Toward a Model for Pattern Formation in Ultrathin-Film Binary Mixtures". In: Macromolecules 38.16 (2005), p. 6775.

[69] A. Karim, T.M. Slawecki, S.K. Kumar, J.F. Douglas, S.K. Satija, C.C. Han, T.P. Russell, Y. Liu, R. Overney, J. Sokolov, M.H. Rafailovich. "PhaseSeparation-Induced Surface Patterns in Thin Polymer Blend Films". In: Macromolecules 31.97 (1998), p. 857.

[70] J. Heier, E.J. Kramer, P. Revesz, G. Battistig, and F.S. Bates. "Spinodal Decomposition in a Subsurface Layer of a Polymer Blend Film". In: Macromolecules 32.11 (1999), p. 3758.

[71] H. Wang and R.J. Composto. "Understanding morphology evolution and roughening in phase-separating thin-film polymer blends". In: Europhysics Letters 50.5 (2000), p. 622.

[72] J.-S. Kim, P.K.H. Ho, C.E. Murphy, and R.H. Friend. "Phase Separation in Polyfluorene-Based Conjugated Polymer Blends: Lateral and Vertical Analysis of Blend Spin-Cast Thin Films". In: Macromolecules 37.8 (2004), p. 2861.

[73] S. Ebbens, R. Hodgkinson, A.J. Parnell, A.D.F. Dunbar, S.J. Martin, P.D. Topham, N. Clarke, and J.R. Howse. "In situ imaging and height reconstruction of phase separation processes in polymer blends during spin coating". In: ACS nano 5.6 (2011), p. 5124.

[74] R. Craster and O. Matar. "Dynamics and stability of thin liquid films". In: Reviews of Modern Physics 81.3 (2009), p. 1131.

[75] M. Geoghegan. "Wetting at polymer surfaces and interfaces". In: Progress in Polymer Science 28.2 (2003), p. 261.

[76] K.R. Thomas, N. Clarke, R. Poetes, M. Morariu, U. Steiner. "Wetting induced instabilities in miscible polymer blends". In: Soft Matter 6.15 (2010), p. 3517 . 
[77] N. Clarke. "Instabilities in thin-film binary mixtures". In: The European Physical Journal E, Soft matter 14.3 (2004), p. 207.

[78] L. Náraigh and J.-L. Thiffeault. "Dynamical effects and phase separation in cooled binary fluid films". In: Physical Review E 76.3 (2007), p. 035303.

[79] A. Pototsky, M. Bestehorn, D. Merkt, and U. Thiele. "Morphology changes in the evolution of liquid two-layer films". In: The Journal of Chemical Physics 122.22 (2005), p. 224711.

[80] A. Pototsky, M. Bestehorn, D. Merkt, and U. Thiele. "Evolution of interface patterns of three-dimensional two-layer liquid films". In: Europhysics Letters 74.4 (2006), p. 665.

[81] O.A. Frolovskaya, A.A. Nepomnyashchy, A. Oron, and A.A. Golovin. "Stability of a two-layer binary-fluid system with a diffuse interface". In: Physics of Fluids 20.11 (2008), p. 112105.

[82] S. Madruga and U. Thiele. "Decomposition driven interface evolution for layers of binary mixtures. II. Influence of convective transport on linear stability". In: Physics of Fluids 21.6 (2009), p. 062104.

[83] H. Morita, T. Kawakatsu, and M. Doi. "Dynamic Density Functional Study on the Structure of Thin Polymer Blend Films with a Free Surface". In: Macromolecules 34 (2001), p. 8777.

[84] U. Thiele, D.V. Todorova, and H. Lopez. "Gradient Dynamics Description for Films of Mixtures and Suspensions: Dewetting Triggered by Coupled Film Height and Concentration Fluctuations". In: Physical Review Letters 111.11 (2013), p. 117801.

[85] D.T.W. Toolan, E. ul Haq, A. Dunbar, S. Ebbens, N. Clarke, P.D. Topham, and J.R. Howse. "Direct observation of morphological development during the spin-coating of polystyrene-poly(methyl methacrylate) polymer blends". In: Journal of Polymer Science Part B: Polymer Physics 51.11 (2013), p. 875 . 